\title{
ANNUAL YIELD AND SELECTED HYDROLOGIC DATA FOR THE ARKANSAS RIVER BASIN COMPACT, ARKANSAS-OKLAHOMA, 1995 WATER YEAR
}

by J.E. Porter

\section{U.S. GEOLOGICAL SURVEY Open-File Report 96-441}

Prepared in cooperation with the ARKANSAS RIVER COMPACT COMMISSION ARKANSAS-OKLAHOMA

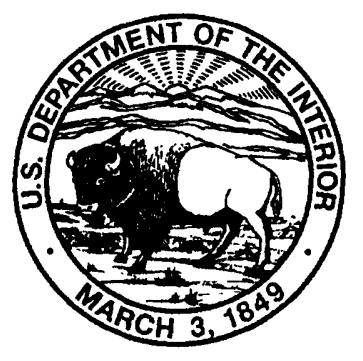

Little Rock, Arkansas 1996 


\section{U.S. DEPARTMENT OF THE INTERIOR \\ BRUCE BABBITT, Secretary}

\section{U.S. GEOLOGICAL SURVEY}

Gordon P. Eaton, Director

For additional information write to:

District Chief

U.S. Geological Survey, WRD

401 Hardin Road

Little Rock, AR 72211
Copies of this report can be purchased from:

U.S. Geological Survey

Branch of Information Services

Box 25286

Denver, CO 80225-0286 


\section{CONTENTS}

Abstract

\section{ILLUSTRATIONS}

Figure 1. Map showing Arkansas-Oklahoma Arkansas River Basin Compact area and subbasins

\section{TABLES}

Table 1. Annual yield and deficiency for the subbasins for the 1995 water year, as defined in the Arkansas River Basin Compact, Arkansas-Oklahoma, 1972 ..................................................... 5

2. Actual runoff from the subbasins for the 1995 water year.................................................................. 5

3. Annual depletion caused by major reservoirs for the 1995 water year ................................................. 6

\section{CONVERSION FACTORS}

\begin{tabular}{lcl}
\multicolumn{1}{c}{ Multiply } & By & To obtain \\
inch (in.) & 25.4 & millimeter \\
foot (ft) & 0.3048 & meter \\
mile (mi) & 1.609 & kilometer \\
acre & 4,047 & square meter \\
& 0.004047 & square kilometer \\
square mile $\left(\mathrm{mi}^{2}\right)$ & 2.590 & square kilometer \\
cubic foot $\left(\mathrm{ft}^{3}\right)$ & 0.02832 & cubic meter \\
acre-foot $(\mathrm{acre}-\mathrm{ft})$ & 1,233 & cubic meter \\
& $1.233 \times 10^{-6}$ & cubic kilometer \\
cubic foot per second $\left(\mathrm{ft}^{3} / \mathrm{s}\right)$ & 28.32 & liter per second \\
& 0.02832 & cubic meter per second \\
ton per day (ton/d) & 0.9072 & megagram per day
\end{tabular}

Temperature in degrees Celsius $\left({ }^{\circ} \mathrm{C}\right)$ can be converted to degrees Fahrenheit $\left({ }^{\circ} \mathrm{F}\right)$ as follows:

$$
{ }^{\circ} \mathrm{F}=1.8 \times{ }^{\circ} \mathrm{C}+32
$$




\title{
ANNUAL YIELD AND SELECTED HYDROLOGIC DATA FOR THE ARKANSAS RIVER BASIN COMPACT, ARKANSAS-OKLAHOMA, 1995 WATER YEAR
}

\author{
By J.E. Porter
}

\begin{abstract}
The computed annual yield and deficiency of the subbasins as defined in the Arkansas River Basin Compact, Arkansas-Oklahoma, are given in tables for the 1995 water year. Actual runoff from the subbasins and depletion caused by major reservoirs in the compact area also are given in tabular form. Monthly mean discharges are shown for the 17 streamflow stations used in computing annual yield. Water-quality data are shown for 20 water-quality stations sampled in the Arkansas River Basin.
\end{abstract}

\section{INTRODUCTION}

In 1955, the Congress of the United States granted consent to Arkansas and Oklahoma to enter into a compact for the apportionment of the waters of the Arkansas River and its tributaries as they affect the two States. An ArkansasOklahoma Arkansas River Compact committee was created with a Federal Representative acting as chairman. After research and deliberate negotiations had been completed, both States approved the Arkansas River Basin Compact, Arkansas-Oklahoma, 1972. To meet the requirements of the Compact, Stateline yields of the Arkansas River Basin are determined at the end of each year.

This report was prepared by the U.S. Geological Survey in cooperation with the Arkansas River Compact Commission, Arkansas-Oklahoma. Streamflow data and water-quality data were collected by the U.S. Geological Survey in cooperation with the Arkansas Soil and Water Conservation Commission and the Oklahoma Water Resources Board. Additional Water-quality data were collected as part of the U.S. Geological Survey's National Water-Quality Assessment Program. The U.S. Army Corps of Engineers, Tulsa District furnished data from the Webbers Falls, Tenkiller Ferry, Robert S. Kerr, Wister, and Fort Gibson Lakes.

\section{PURPOSE AND SCOPE}

The purpose of this report is to present the annual yields and deficiencies computed for the 1995 water year for subbasins in the Arkansas River Basin as defined in the Arkansas River Basin Compact, Arkansas-Oklahoma, 1972. The report includes data from 17 streamflow stations and 20 water-quality stations sampled in the Arkansas River Basin during the 1995 water year. The area included in the Compact is shown on figure 1 .

\section{DEFINITION OF TERMS}

The following terms used in this report are taken from Article II of the Arkansas River Basin Compact, ArkansasOklahoma, 1972.

The term "Arkansas River Basin" means all of the drainage basin of the Arkansas River and its tributaries from a point immediately downstream from the confluence of the Neosho River with the Arkansas River (fig. 1) to a point immediately downstream from the confluence of Lee Creek with the Arkansas River, together with the drainage basin of Spavinaw Creek in Arkansas (fig. 1), but excludes that part of the drainage basin of the Canadian River upstream from Lake Eufaula Dam.

The term "Spavinaw Creek Subbasin" means the drainage area of Spavinaw Creek in the State of Arkansas.

The term "Illinois River Subbasin" means the drainage area of the Illinois River in the State of Arkansas.

The term "Lee Creek Subbasin" means the drainage area of Lee Creek in the State of Arkansas and in the State of Oklahoma.

The term "Poteau River Subbasin" means the drainage area of the Poteau River in the State of Arkansas.

The term "Arkansas River Subbasin" means all areas of the Arkansas River Basin except the four subbasins described previously. 


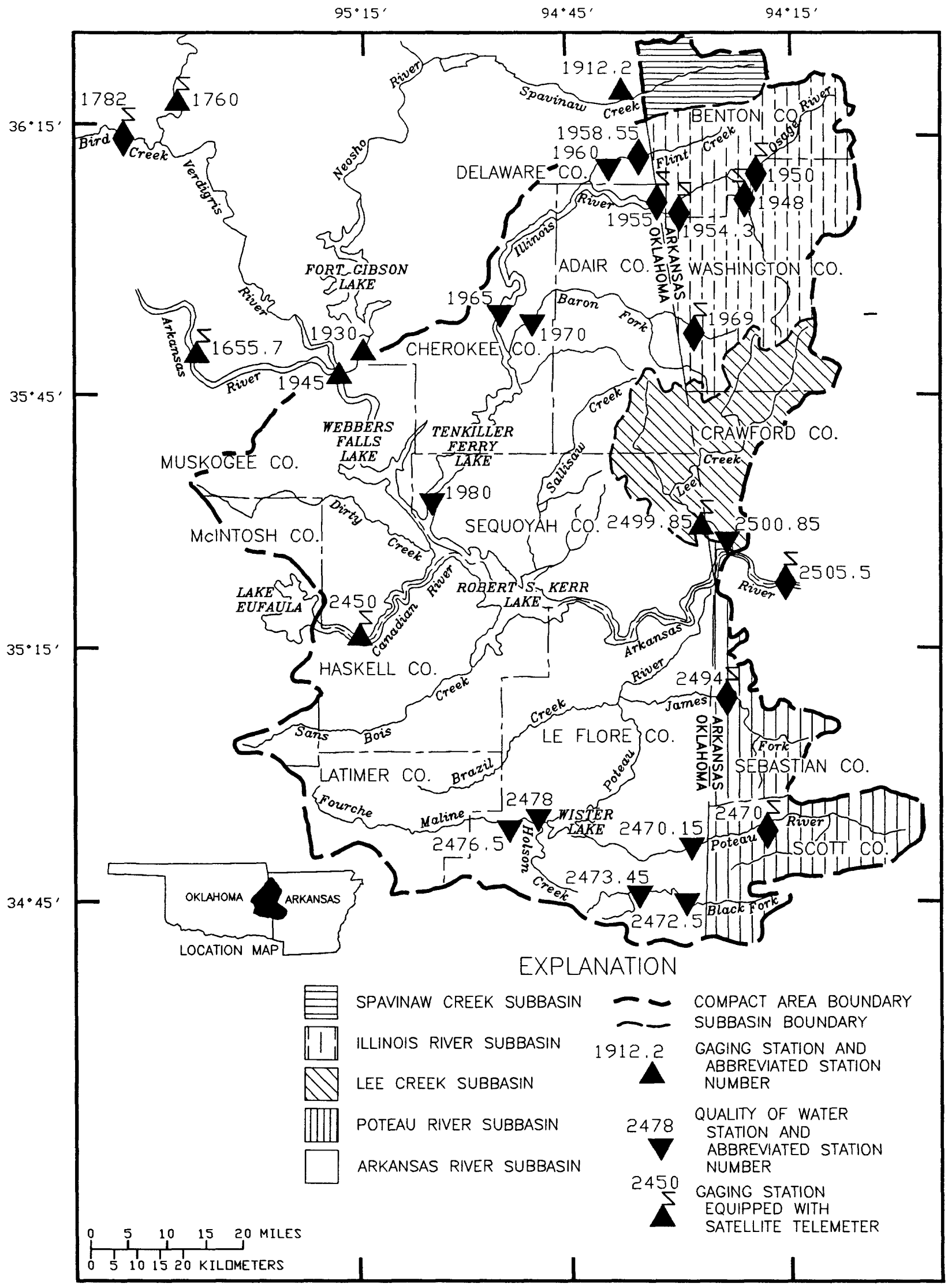

Figure 1.--Arkansas-Oklahoma Arkansas River Basin Compact area and subbasins. 
The term "water year" means a 12-month period beginning on October 1 and ending September 30.

The term "annual yield" means the computed annual gross runoff from any specified subbasin. The runoff would have passed any certain point on a stream and would have originated within any specified area under natural conditions without any manmade depletion or accretion during the water year.

Terms related to streamflow, water-quality, and other hydrologic data, as used in this report, are defined below.

Acre-foot is the quantity of water required to cover 1 acre to a depth of 1 foot and is equivalent to 43,560 cubic feet.

Bacteria are microscopic unicellular organisms, typically spherical, rodlike, or spiral and threadlike in shape, often clumped into colonies. Some bacteria cause disease, others perform an essential role in nature in the recycling of materials; for example, by decomposing organic matter into a form available for reuse by plants.

Escherichia coli (E. coli) also are present in the digestive tract of warm-blooded animals. In the laboratory, E. coli is defined as all organisms that produce orange/yellow when incubated for two hours at $35^{\circ} \mathrm{C} \pm$ $0.2^{\circ} \mathrm{C}$ and transferred to $44.5^{\circ} \pm 0.2^{\circ}$ for $22-24$ hours on mTEC agar (nutrient medium for $E$. coli growth), and strained with phenol red solution. Their concentrations are expressed as number of colonies per $100 \mathrm{~mL}$ of sample.

Fecal coliform bacteria are present in the intestines or feces of warm-blooded animals. They are often used as indicators of the sanitary quality of the water. In the laboratory, they are defined as all the organisms that produce blue colonies within 24 hours when incubated at $44.5^{\circ} \mathrm{C} \pm 0.2^{\circ} \mathrm{C}$ on $\mathrm{M}-\mathrm{FC}$ medium (nutrient medium for bacterial growth). Their concentrations are expressed as number of colonies per 100 milliliters $(\mathrm{mL})$ of sample.

Fecal streptococcal bacteria also are present in intestines of warm-blooded animals. Their presence in water is considered to verify fecal pollution. They are characterized as grampositive, cocci bacteria that are capable of growth in brain-heart infusion broth. These bacteria also are defined as all the organisms that produce red or pink colonies within 48 hours at $35^{\circ} \mathrm{C} \pm 0.5^{\circ} \mathrm{C}$ on KF-streptococcus agar (nutrient medium for bacterial growth). Their concentrations are expressed as number of colonies per $100 \mathrm{~mL}$ of sample.

Code numbers have been assigned for agencies collecting and analyzing samples, and are listed in waterquality tables of this report as follows:

1028 Oklahoma District, Water Resources Division (WRD), U.S. Geological Survey

80513 Arkansas District, WRD, U.S. Geological Survey

80020 National Water Quality Laboratory, WRD, U.S. Geological Survey.

Contents are the volume of water in a reservoir or lake. Unless otherwise indicated, volume is computed on the basis of a level pool and does not include bank storage.

Cubic foot per second is the rate of discharge representing a volume of 1 cubic foot passing a specified point during 1 second.

Deficiency is the amount the actual runoff is less than the minimum required flow.

Depletion caused by major reservoirs is the difference between the inflow and outflow in the reservoirs.

Discharge is the volume of water that passes a given point within a given period of time.

Instantaneous discharge is the discharge at a particular instant of time.

Mean discharge is the arithmetic average of individual daily mean discharges during a specific period.

Dissolved refers to the material in a representative water sample that passes through a 0.45 -micron membrane filter. This is a convenient operational definition used by Federal agencies that collect water data. Determinations of "dissolved" constituents are made on subsamples of the filtrate.

Dissolved oxygen content of water in equilibrium with air is a function of atmospheric pressure and temperature and the dissolved-solids concentration of the water. The ability of water to retain oxygen decreases with increasing temperature or dissolved solids, with small temperature changes having the more significant effect. Photosynthesis and respiration may cause diurnal variations in dissolved-oxygen concentration in water of some streams.

Drainage area of a stream at a specified location is that area, measured in a horizontal plane, enclosed by a topographic divide from which direct surface runoff from precipitation normally drains by gravity into the stream upstream from the specified point. Figures of drainage area given herein include all closed basins, or noncontributing areas within the area, unless otherwise noted. 
Gaging station is a particular site on a stream, canal, lake, or reservoir where systematic observations of hydrologic data are obtained.

Hardness of water is a physical-chemical characteristic that is commonly recognized by the increased quantity of soap required to produce lather. It is attributable to the presence of alkaline earths (principally calcium and magnesium) and is expressed as equivalent calcium carbonate $\left(\mathrm{CaCO}_{3}\right)$.

Sediment is solid material that originates mostly from disintegrated rocks and is transported by, suspended in, or deposited from water; it includes chemical and biochemical precipitates and decomposed organic material, such as humus. The quantity, characteristics, and cause of the occurrence of sediment in streams are influenced by environmental factors. Some major factors are degree of slope, length of slope, soil characteristics, land usage, and quantity and intensity of precipitation.

Mean concentration is the time-weighted concentration of suspended sediment passing a stream cross section during a 24-hour period.

Suspended sediment is the sediment that at any given time is maintained in suspension by the upward components of turbulent currents or that exists in suspension as a colloid.

Suspended-sediment concentration is the velocity-weighted concentration of suspended sediment in the sampled zone (from the water surface to a point approximately $0.3 \mathrm{ft}$ above the bed), expressed as milligrams of dry sediment per liter of water-sediment mixture $(\mathrm{mg} / \mathrm{L})$.

Suspended-sediment discharge (tons/day) is the rate at which dry weight of sediment passes a section of a stream or is the quantity of sediment, as measured by dry weight or volume, that passes a section in a given time. It is computed by multiplying discharge by milligrams per liter by 0.0027 .

Sodium-absorption-ratio (SAR) is the expression of relative activity of sodium ions in exchange reactions with soil and is an index of sodium or alkali hazard to the soil. Water varies, in respect to sodium hazard, from that which can be used for irrigation on almost all soils to that which generally is unsatisfactory for irrigation.

Specific conductance is a measure of the ability of water to conduct an electrical current. It is expressed in microsiemens per centimeter at $25^{\circ} \mathrm{C}$. Specific conductance is related to the type and concentration of ions in solution and can be used for approximating the dissolved-solids concentration of the water. Commonly, the concentration of dissolved solids (in milligrams per liter) is about 65 percent of the specific conductance (in microsiemens). This relation is not constant from stream to stream, and it may vary in the same source with changes in the composition of the water.

Stage-discharge relation is the relation between gage height (stage) and the volume of water, per unit of time, flowing past the gage in a channel.

STORET parameter codes are codes assigned to specific hydrologic measurement types for computer storage of data. These five-digit codes (shown in parentheses) are included with the water-quality information in the Hydrologic Station Records section.

Streamflow is the discharge that occurs in a natural channel. Although the term "discharge" can be applied to the flow of a canal, the word "streamflow" uniquely describes the discharge in a surface stream course. The term "streamflow" is more general than "runoff", as streamflow may be applied to discharge whether or not it is affected by diversion or regulation.

Tons per day is the quantity of substance in solution or suspension that passes a stream section during a 24-hour period.

Total is the total amount of a given constituent in a representative water-suspended sediment sample, regardless of the constituent's physical or chemical form. This term is used only when the analytical procedure assures measurement of at least 95 percent of the constituent present in both the dissolved and suspended phases of the sample. A knowledge of the expected form of the constituent in the sample, as well as the analytical methodology used, is required to judge when the results should be reported as "total." (Note that the word "total" does double duty here, indicating that the sample consists of a water-suspended-sediment mixture and that the analytical method determines all of the constituent in the sample.) 


\section{COMPUTATION OF ANNUAL YIELDS}

The annual yield and deficiency (table 1) for each subbasin were computed as described in Appendix I to the Arkansas River Basin Compact, Arkansas-Oklahoma, 1972, supplement No. 1. Actual runoff for the subbasins (table 2) was computed as described in the Compact except for the stations Arkansas River at Muskogee, which has been discontinued, Arkansas River at Van Buren, which has been moved 7.9 miles downstream, and Lee Creek near Van Buren, which has been moved 3.2 miles upstream to near Short, Oklahoma.

\section{Table 1.--Annual yield and deficiency for the subbasins for the 1995 water year, as defined in the Arkansas River Basin Compact, Arkansas-Oklahoma, 1972}

[Flow in cubic feet per second]

\begin{tabular}{lcccccc}
\hline Subbasin & $\begin{array}{c}\text { Actual runoff } \\
\text { from the } \\
\text { subbasins }\end{array}$ & $\begin{array}{c}\text { Total } \\
\text { depletions or } \\
\text { accretions (-) }\end{array}$ & Annual yield & $\begin{array}{c}{ }^{\mathrm{a}} \text { Percent } \\
\text { depletion } \\
\text { allowed }\end{array}$ & $\begin{array}{c}\text { Minimum } \\
\text { required flow }\end{array}$ & beficiency \\
\hline Spavinaw Creek & 170 & ${ }^{c} 0.8$ & 171 & 50 & 86 & 0 \\
Illinois River & 1,050 & ${ }^{c} 495$ & 1,545 & 60 & 618 & 0 \\
Lee Creek & 728 & $c^{c} 24.9$ & 753 & 100 & 0 & 0 \\
Poteau River & 814 & $c 2.0$ & 816 & 60 & 326 & 0 \\
Arkansas River & 5,250 & $\mathrm{~d} 264$ & 5,514 & 60 & 2,206 & 0 \\
\hline
\end{tabular}

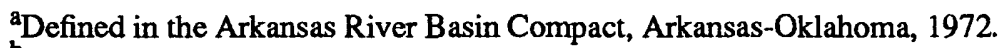

b The amount the actual runoff is less than the minimum required flow.

${ }^{c}$ Based on 1994 water year water-use data.

${ }^{\mathrm{d}}$ Based on 1994 water-use data and 1995 direct diversions from lake storage.

Table 2.--Actuai runoff from the subbasins for the 1995 water year

[In cubic feet per second; D.A. = drainage area; $\mathrm{mi}^{2}=$ square mile; acre-ft = acre-feet]

\begin{tabular}{lccccc}
\hline Month & $\begin{array}{c}\text { Spavinaw Creek } \\
\text { ad.A. }=135 \mathrm{mi}^{2}\end{array}$ & $\begin{array}{c}\text { Illinois River } \\
\text { b D.A. }=744 \mathrm{mi}^{2}\end{array}$ & $\begin{array}{c}\text { Lee Creek } \\
\text { D.A. }=426 \mathrm{mi}^{2}\end{array}$ & $\begin{array}{c}\text { Poteau River } \\
{ }^{\mathrm{C}} \mathrm{D} . \mathrm{A} .=536 \mathrm{mi}^{2}\end{array}$ & $\begin{array}{c}\text { Arkansas River } \\
\mathrm{d} \text { D.A. }=4,591 \mathrm{mi}^{2}\end{array}$ \\
\hline October & 24.3 & 169 & 25.6 & 21.1 & 124 \\
November & 186 & 1,330 & 1,680 & 2,690 & 12,360 \\
December & 83.7 & 780 & 784 & 1,370 & 13,600 \\
January & 200 & 1,860 & 1,370 & 1,910 & 13,050 \\
February & 95.8 & 638 & 375 & 251 & 4,595 \\
March & 142 & 962 & 758 & 414 & 2,732 \\
April & 234 & 1,490 & 1,160 & 810 & 5,935 \\
May & 405 & 2,540 & 1,640 & 1,740 & 7,085 \\
June & 494 & 1,880 & 771 & 448 & 0 \\
July & 93.2 & 472 & 136 & 58.3 & 3,317 \\
August & 50.0 & 225 & 17.0 & 9.22 & 0 \\
September & 35.1 & 204 & 8.52 & 12.6 & 0 \\
\hline 1995 water year & 170 & 1,050 & 728 & 814 & 5,246 \\
1995 water year (acre-ft) & 123,000 & 761,000 & 527,000 & 590,000 & $3,801,000$ \\
\hline
\end{tabular}

${ }^{\text {a Includes }} 31 \mathrm{mi}^{2}$ ungaged.

bincludes $63 \mathrm{mi}^{2}$ ungaged.

' Includes $125 \mathrm{mi}^{2}$ ungaged.

${ }^{d}$ Computed by subtracting drainage area at Arkansas River at Muskogee, Canadian River near Whitefield, Illinois River Subbasin, Lee Creek Subbasin, and Poteau River Subbasin from drainage area at Arkansas River at James W. Trimble Dam near Van Buren, Arkansas. 
Annual depletion caused by major reservoirs (table 3) was computed for the four major reservoirs in the basin as described in Appendix I to the Compact. Depletions caused by small reservoirs and minor diversions for municipal and agricultural use are considered by subbasins in table 1.

A compilation of the areas and capacities of lakes and ponds in Arkansas (Arkansas Soil and Water Conservation Commission, 1981) was used to evaluate depletions caused by small reservoirs in the Poteau River, Lee Creek, Spavinaw Creek, and Illinois River subbasins. Analysis indicated that their impact on the depletions in any subbasin, except Illinois River, was probably insignificant. Information on depletions continue to be gathered in order to reevaluate their present impact.

Streamflow data used in the computations are given in hydrologic station records (p. 8 to 63). The station description under "Remarks" states the degree of accuracy of the records. "Excellent" means that about 95 percent of the daily discharges are within 5 percent of the actual discharge, "good" means within 10 percent, and "fair" means within 15 percent. "Poor" means that daily discharges have less than "fair" accuracy.

Table 3.--Annual depletlon caused by major reservolrs for the 1995 water year

$$
\text { [acre-ft = acre-feet; } \mathrm{ft}^{3} / \mathrm{s}=\text { cubic feet per second] }
$$

\begin{tabular}{|c|c|c|c|c|c|c|c|}
\hline Reservoir & $\begin{array}{l}\text { Year-end } \\
\text { contents } \\
\text { (acre-ft) }\end{array}$ & $\begin{array}{c}\text { Change in } \\
\text { contents in } \\
\text { water year } \\
\text { (acre-ft) }\end{array}$ & $\begin{array}{c}{ }^{\text {a }} \text { Precipitation } \\
\text { on reservoir } \\
\text { surface } \\
\text { (inches) }\end{array}$ & $\begin{array}{c}{ }^{\mathrm{b}} \text { Evaporation } \\
\text { from } \\
\text { reservoir } \\
\text { (inches) }\end{array}$ & $\begin{array}{l}{ }^{\text {a Diversions }} \\
\text { (acre-ft) }\end{array}$ & $\begin{array}{c}\text { Depletion } \\
\text { (acre-ft) }\end{array}$ & $\begin{array}{c}\text { Depletion } \\
\left(\mathrm{ft}^{3} / \mathrm{s}\right)\end{array}$ \\
\hline Webbers Falls & 169,000 & $+5,230$ & 49.79 & 69.08 & 0 & $+19,610$ & +27.1 \\
\hline Tenkiller Ferry & 656,600 & $+14,800$ & 43.61 & 55.56 & 6,230 & $+29,690$ & +41.0 \\
\hline Robert S. Kerr & 523,500 & $+5,600$ & 51.57 & 63.88 & 0 & $+47,120$ & +65.0 \\
\hline Wister & 47,200 & $-12,870$ & 50.32 & 53.16 & 9,540 & +200 & +0.3 \\
\hline
\end{tabular}

${ }^{\mathrm{a}}$ From U.S. Corps of Engineers, Tulsa District.

bAdjusted for pan coefficient of 0.70 (from Wisler and Brater, 1949). 


\section{SELECTED REFERENCES}

Arkansas River Compact Committee, 1972, Arkansas River Basin Compact Arkansas-Oklahoma, 1972, with Supplemental Interpretive Comments, Supplement No. 1: Austin, Texas, 31 p.

Arkansas Soil and Water Conservation Commission, 1981, Arkansas State Water Plan - Lakes of Arkansas, 157 p.

Wisler, C.D., and Brater, E.F., 1949, Hydrology: New York, John Wiley \& Sons, Inc., 150 p. 

HYDROLOGIC STATION RECORDS 


\section{ABBREVIATIONS}

AC-FT

COLS . /100ML

DEG. C

FLTRD (other abreviations sometimes used)

FT FM L BANK

INST

K

$\mathrm{MG} / \mathrm{L}$

$\boldsymbol{\mu}$

$\mu G / L$

ML

MM

NIU

REC

UM-MF

T/DAY

$\mathrm{US} / \mathrm{CM}$

$<$
Acre-foot

Number of colonies per 100 milliliters

Degrees Celsius

Filtered

Feet from left bank

Instantaneous

Non-ideal count

Milligrams per liter

Micron

Micrograms per liter

Milliliters

Millimeters

Nephelemetric turbidity units

Recoverable

Micron membrane filter

Tons per day

Microsiemens per centimeter at 25 degrees Celsius Less than

No data available 


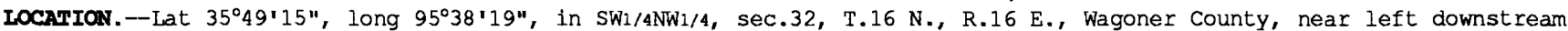
abutment of old bridge downstream from state Highway 104, 2.0 mi east of Haskell, 23.5 mi upstream from Verdigris River, and at mile 483.7 .

DRATNAGs AREA. $-75,473 \mathrm{mi}^{2}$, of which $12,541 \mathrm{mi}^{2}$ probably is noncontributing.

AVRRACE DIScharee. -23 years, $10,340 \mathrm{ft}^{3} / \mathrm{s}$.

Exuremgs.--June 1972 to current year: Maximum discharge 259,000 $\mathrm{ft}^{3} / \mathrm{s}$ oct. 5, 1986; minimum daily $87 \mathrm{ft}{ }^{3} / \mathrm{s} \mathrm{Sept.} 13$, 1988 . RERarRs.--Records fair. Flow regulated by Keystone Lake, $55.1 \mathrm{mi}$ upstream. U.S. Army Corps of Englneers' satell1te telemeter at station.

Monthly and yearly discharge

\begin{tabular}{|c|c|c|c|c|c|}
\hline Month & $\begin{array}{l}\text { Total } \\
\left(\mathrm{ft}^{3} / \mathrm{s}\right)\end{array}$ & $\begin{array}{c}\text { Maximum } \\
\text { daily } \\
\left(\mathrm{ft}^{3} / \mathrm{s}\right)\end{array}$ & $\begin{array}{l}\text { Minimum } \\
\text { daily } \\
\left(\mathrm{ft}^{3} / \mathrm{s}\right)\end{array}$ & $\underset{\left(f t^{3} / \mathrm{s}\right)}{\operatorname{Mean}}$ & $\begin{array}{c}\text { Runoff } \\
\text { (acre-feet) }\end{array}$ \\
\hline October & 39,985 & 3,770 & 498 & 1,290 & 79,310 \\
\hline November & 243,687 & 23,000 & 527 & 8,123 & 483,400 \\
\hline December & 222,900 & 15,200 & 1,000 & 7,190 & 442,100 \\
\hline January & 120,940 & 7,590 & 1,380 & 3,901 & 239,900 \\
\hline February & 89,949 & 11,000 & 969 & 3,212 & 178,400 \\
\hline March & 488,400 & 31,100 & 1,470 & 15,750 & 968,700 \\
\hline April & 259,730 & 24,700 & 1,800 & 8,658 & 515,200 \\
\hline May & 953,000 & 57,700 & 17,100 & 30,740 & $1,890,000$ \\
\hline June & $2,354,400$ & 138,000 & 46,100 & 78,480 & $4,670,000$ \\
\hline July & 965,100 & 50,200 & 13,200 & 31,130 & $1,914,000$ \\
\hline August & $1,008,700$ & 42,700 & 11,800 & 32,540 & $2,001,000$ \\
\hline September & 248,150 & 15,300 & 3,290 & 8,272 & 492,200 \\
\hline Water year 1995 & $6,994,941$ & 138,000 & 498 & 19,160 & $13,870,000$ \\
\hline
\end{tabular}




\section{ARKANSAS RIVER SUBBASIN}

\section{VRRDIGRIS RIVER IRAR CLAREMORR, OKIABOMA}

LOCATIOX.--Lat $36^{\circ} 18^{\prime} 26^{\prime \prime}$, long $95^{\circ} 41 \cdot 52^{\prime \prime}$, in NE1/4NW1/4, sec.15, T.21 N., R.15 E., Rogers County, on left bank on downstream side of bridge on State Highway $20,2.3 \mathrm{mi}$ downstream from Caney River, $4.5 \mathrm{mi}$ west of Claremore, $12.4 \mathrm{mi}$ upstream from Bird Creek, and at mile 76.0.

DRATIAEE AREA. $--6,534 \mathrm{mi}^{2}$.

AVERACE DIScEARE. --27 years (water years 1936-62), 3,723 $\mathrm{ft}^{3} / \mathrm{s} ; 31$ years (water years $1965-95$ ), 4,626 ft $3 / \mathrm{s}$.

EXIREMS.--October 1935 to current year: Maximum discharge 182,000 ft³ $/ \mathrm{s}$ May 21, 1943; no flow at times in 1936, 1939$40,1956$.

RERRRKs.--Records good. Flow regulated since May 1963 by Oologah Lake $14.3 \mathrm{mi}$ upstream; some regulation by dams in Kansas since 1949 and by Hulah Lake since 1950. U.S. Army Corps of Engineers' satellite telemeter at station.

Monthly and yearly discharge

\begin{tabular}{|c|c|c|c|c|c|}
\hline Month & $\begin{array}{l}\text { Total } \\
\left(\mathrm{ft}^{3} / \mathrm{s}\right)\end{array}$ & $\begin{array}{l}\text { Maximum } \\
\mathrm{dail}^{2} \mathrm{l} \\
\left(\mathrm{ft}^{3} / \mathrm{s}\right)\end{array}$ & $\begin{array}{l}\text { Minimum } \\
\text { daily } \\
\left(\mathrm{ft}^{3} / \mathrm{s}\right)\end{array}$ & $\begin{array}{c}\text { Mean } \\
\left(\mathrm{ft}^{3} / \mathrm{s}\right)\end{array}$ & $\begin{array}{c}\text { Runoff } \\
(\text { acre-feet })\end{array}$ \\
\hline October & 12,297 & 3,180 & 82 & 397 & 24,390 \\
\hline November & 209,241 & 13,600 & 128 & 6,975 & 415,000 \\
\hline December & 149,704 & 11,200 & 389 & 4,829 & 296,900 \\
\hline January & 20,902 & 1,250 & 363 & 674 & 41,460 \\
\hline February & 18,129 & 1,290 & 287 & 647 & 35,960 \\
\hline March & 162,149 & 14,800 & 506 & 5,231 & 321,600 \\
\hline April & 154,876 & 21,400 & 328 & 5,163 & 307,200 \\
\hline May & 667,700 & 31,000 & 12,600 & 21,540 & $1,324,000$ \\
\hline June & 761,100 & 34,900 & 14,800 & 25,370 & $1,510,000$ \\
\hline July & 692,500 & 40,300 & 11,300 & 22,340 & $1,374,000$ \\
\hline August & 225,793 & 15,300 & 148 & 7,284 & 447,900 \\
\hline September & 8,160 & 1,180 & 142 & 272 & 16,190 \\
\hline Water year 1995 & $3,082,551$ & 40,300 & 82 & 8,445 & $6,114,000$ \\
\hline
\end{tabular}




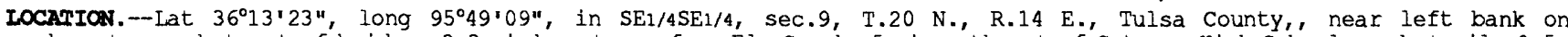
downstream abutment of bridge, $2.3 \mathrm{mi}$ downstream from Elm Creek, $5 \mathrm{mi}$ northwest of Catoosa High School, and at mile 9.5 . DRATIAGE AREA. $--1,103 \mathrm{mi}^{2}$.

AVERAER DISCEARES. -7 years, $1,193 \mathrm{ft}^{3} / \mathrm{s}$.

EXIRFres.--August 1988 to current year: Maximum discharge $27,400 \mathrm{ft}^{3} / \mathrm{s}$ May 11, 1993, gage height, $33.22 \mathrm{ft}$; minimum dally discharge $62 \mathrm{ft}^{3} / \mathrm{s}$ Nov. 6, 1993 .

RERaRKs.--Records fair. Some regulation by skiatook Lake (station 07177400). U.S. Geological Survey's satellite telemeter at station.

Monthly and yearly discharge

\begin{tabular}{|c|c|c|c|c|c|}
\hline Month & $\begin{array}{l}\text { Total } \\
\left(\mathrm{ft}^{3} / \mathrm{s}\right)\end{array}$ & $\begin{array}{l}\text { Maximum } \\
\text { daily } \\
\left(\mathrm{ft}^{3} / \mathrm{s}\right)\end{array}$ & $\begin{array}{c}\text { Minimum } \\
\text { daily } \\
\left(\mathrm{ft}^{3} / \mathrm{s}\right)\end{array}$ & $\underset{\left(\mathrm{ft}^{3} / \mathrm{s}\right)}{\operatorname{Mean}}$ & $\begin{array}{c}\text { Runoff } \\
\text { (acre-feet) }\end{array}$ \\
\hline October & 13,859 & 1,660 & 122 & 447 & 27,490 \\
\hline November & 78,078 & 11,70 & 172 & 2,603 & 154,900 \\
\hline December & 11,796 & 1,900 & 200 & 381 & 23,400 \\
\hline January & 7,262 & 353 & 200 & 234 & 14,400 \\
\hline February & 7,114 & 1,430 & 157 & 254 & 14,110 \\
\hline March & 52,653 & 8,930 & 247 & 1,698 & 104,400 \\
\hline April & 93,444 & 10,300 & 216 & 3,115 & 185,300 \\
\hline May & 177,440 & 18,600 & 2,890 & 5,724 & 352,000 \\
\hline June & 169,746 & 16,500 & 726 & 5,658 & 336,700 \\
\hline July & 99,047 & 12,800 & 263 & 3,195 & 196,500 \\
\hline August & 14,165 & 1,720 & 228 & 457 & 28,100 \\
\hline September & 13,975 & 1,610 & 222 & 466 & 27,720 \\
\hline Water year 1995 & 738,579 & 18,600 & 122 & 2,024 & $1,465,000$ \\
\hline
\end{tabular}


PERTOD RE RBCORD.--AUGUST 1988 to current year.

PERIOD OF DAILY RBCORD.--

SPECIFIC CONDUCPANCE: August 1988 to current year.

pH: August 1988 to current year.

WATER TERPRRATURR: August 1988 to current year.

DISSOLVED OXYGEN: August 1988 to current year.

INSTrRorasmaticas.--Water-qua11ty monitor since August 1988.

RERRRs.--Interruptions in record were due to malfunction of the recording instrument.

EXITREAYIES FOR PERTOD OF DAIIYY RECORD.--

SPECIFIC CONDCPANCE: Maximum, 1,300 microsiemens, Nov. 4, 1992; minimum 56 microsiemens, July 18 , 1989.

pH: Maximum, 9.4 units, July 17, 1989; minimum 6.0 units, May 12, 1991.

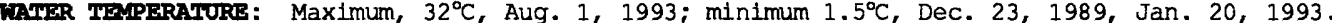

DISSOLVED OXYGEN: Maximum, $14.4 \mathrm{mg} / \mathrm{L}$, Jan. 20, 1992; minimum 2.6 mg/L, Apr. $18,1991$.

EXOIREMIES FOR CURRENT YRAR.--

SPECIFIC CONDOCPANCE: Maximum, 976 microsiemens, Feb. 28; minimum 84 microsiemens, July 4.

pH: Maximum recorded (more than 20 percent missing record), 8.0 units, Oct. 2, Dec. 31, Jan. 1, 2, 7-11, Feb. 22; minimum recorded, 7.0 units, June 12, July 13, Aug. 11, 19, 20.

MATHR TEMPRRATURE: Maximum, $30.5^{\circ} \mathrm{C}$, Aug. 20,21 ; minimum $3.5^{\circ} \mathrm{C}$, Jan. 5.

DISSOLVED OXYGEN: Maximum recorded, $14.3 \mathrm{mg} / \mathrm{L}$, Feb. 22, but may have been higher Feb. $11-21$ when probe falled; minimum recorded, $3.3 \mathrm{mg} / \mathrm{L}$, Sept. 8.

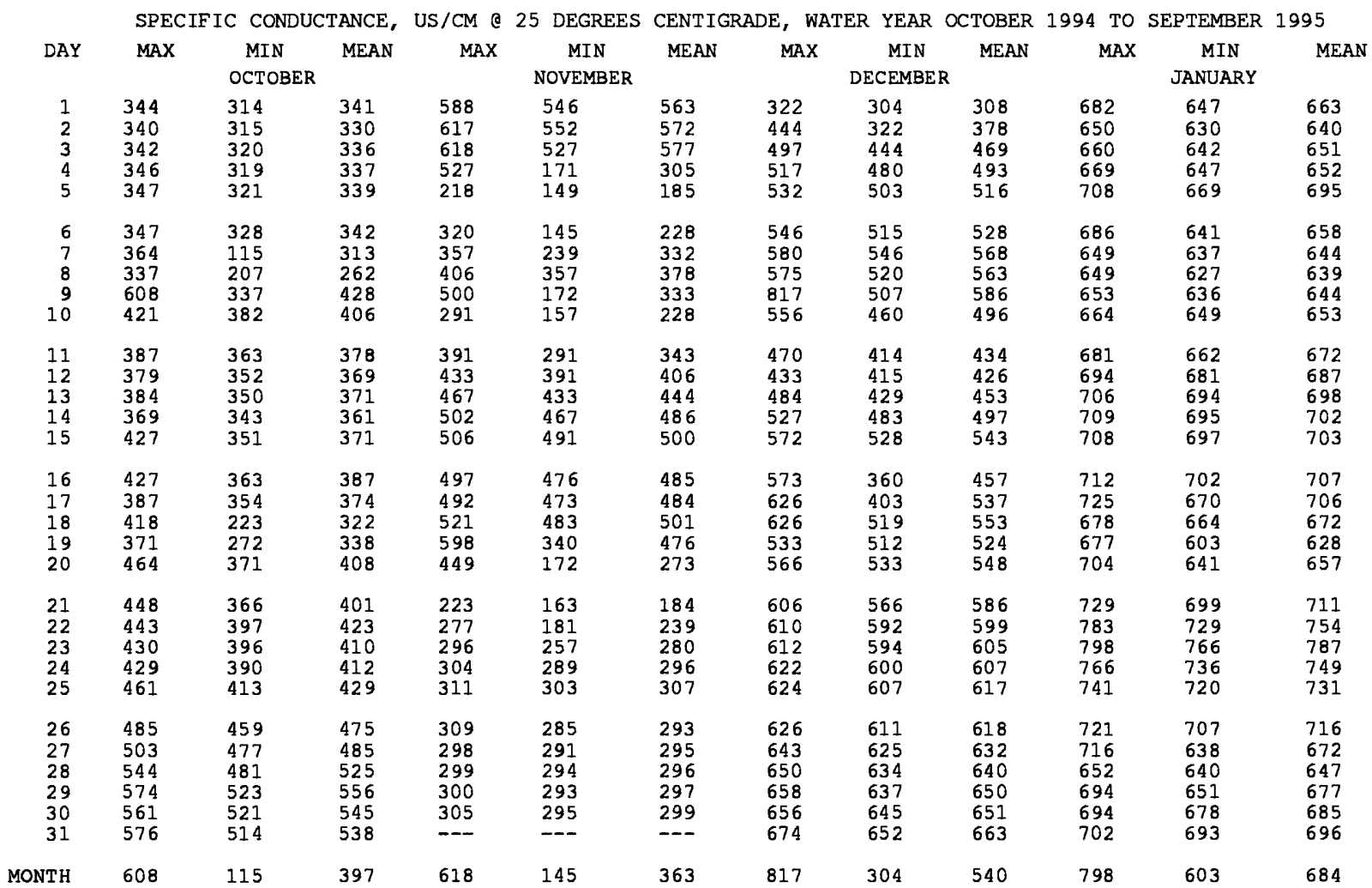


ARKANSAS RTVER SUBBASTN

07178200 BIRD CREEK AT STATE BIGEWAY 266 NEAR CATOOSA, OKLABOMA--CONTINOED

SPECIFIC CONDUCTANCE, US/CM \& 25 DEGREES CENTIGRADE, WATER YEAR OCTOBER 1994 TO SEPTEMBER 1995

\begin{tabular}{|c|c|c|c|c|c|c|c|c|c|c|c|c|}
\hline \multirow[b]{2}{*}{ DAY } & \multicolumn{3}{|c|}{ FEBRUARY } & \multicolumn{3}{|c|}{ MARCH } & \multicolumn{3}{|c|}{ APRIL } & \multicolumn{3}{|c|}{ MAY } \\
\hline & $\operatorname{MAX}$ & MIN & MEAN & MAX & MIN & MEAN & $\operatorname{MAX}$ & MIN & MEAN & $\operatorname{MAX}$ & MIN & MEAN \\
\hline $\begin{array}{l}1 \\
2 \\
3 \\
4 \\
5\end{array}$ & $\begin{array}{l}722 \\
734 \\
720 \\
710 \\
708\end{array}$ & $\begin{array}{l}702 \\
720 \\
705 \\
699 \\
685\end{array}$ & $\begin{array}{l}712 \\
726 \\
714 \\
706 \\
699\end{array}$ & $\begin{array}{l}562 \\
476 \\
447 \\
535 \\
532\end{array}$ & $\begin{array}{l}449 \\
378 \\
372 \\
447 \\
456\end{array}$ & $\begin{array}{l}497 \\
411 \\
384 \\
497 \\
485\end{array}$ & $\begin{array}{l}568 \\
570 \\
583 \\
617 \\
615\end{array}$ & $\begin{array}{l}541 \\
549 \\
570 \\
583 \\
595\end{array}$ & $\begin{array}{l}558 \\
557 \\
575 \\
602 \\
607\end{array}$ & $\begin{array}{l}291 \\
295 \\
329 \\
306 \\
277\end{array}$ & $\begin{array}{l}267 \\
262 \\
276 \\
192 \\
268\end{array}$ & $\begin{array}{l}280 \\
280 \\
292 \\
241 \\
272\end{array}$ \\
\hline $\begin{array}{r}6 \\
7 \\
8 \\
9 \\
10\end{array}$ & $\begin{array}{l}693 \\
684 \\
680 \\
682 \\
707\end{array}$ & $\begin{array}{l}676 \\
675 \\
664 \\
667 \\
682\end{array}$ & $\begin{array}{l}685 \\
681 \\
672 \\
670 \\
698\end{array}$ & $\begin{array}{l}456 \\
508 \\
430 \\
424 \\
445\end{array}$ & $\begin{array}{l}399 \\
400 \\
391 \\
385 \\
395\end{array}$ & $\begin{array}{l}417 \\
435 \\
410 \\
394 \\
417\end{array}$ & $\begin{array}{l}607 \\
596 \\
593 \\
595 \\
582\end{array}$ & $\begin{array}{l}586 \\
577 \\
577 \\
565 \\
416\end{array}$ & $\begin{array}{l}598 \\
589 \\
588 \\
582 \\
550\end{array}$ & $\begin{array}{l}296 \\
269 \\
153 \\
145 \\
222\end{array}$ & $\begin{array}{l}252 \\
145 \\
115 \\
117 \\
145\end{array}$ & $\begin{array}{l}276 \\
208 \\
133 \\
131 \\
179\end{array}$ \\
\hline $\begin{array}{l}11 \\
12 \\
13 \\
14 \\
15\end{array}$ & $\begin{array}{l}722 \\
739 \\
751 \\
754 \\
769\end{array}$ & $\begin{array}{l}700 \\
722 \\
739 \\
748 \\
741\end{array}$ & $\begin{array}{l}713 \\
733 \\
747 \\
750 \\
752\end{array}$ & $\begin{array}{l}444 \\
425 \\
535 \\
349 \\
285\end{array}$ & $\begin{array}{l}415 \\
406 \\
349 \\
217 \\
230\end{array}$ & $\begin{array}{l}425 \\
416 \\
423 \\
262 \\
269\end{array}$ & $\begin{array}{l}574 \\
345 \\
346 \\
351 \\
423\end{array}$ & $\begin{array}{l}237 \\
302 \\
336 \\
337 \\
351\end{array}$ & $\begin{array}{l}312 \\
321 \\
341 \\
343 \\
381\end{array}$ & $\begin{array}{l}286 \\
292 \\
298 \\
299 \\
295\end{array}$ & $\begin{array}{l}222 \\
286 \\
291 \\
293 \\
291\end{array}$ & $\begin{array}{l}265 \\
290 \\
294 \\
295 \\
292\end{array}$ \\
\hline $\begin{array}{l}16 \\
17 \\
18 \\
19 \\
20\end{array}$ & $\begin{array}{l}789 \\
796 \\
790 \\
791 \\
788\end{array}$ & $\begin{array}{l}769 \\
775 \\
778 \\
779 \\
775\end{array}$ & $\begin{array}{l}782 \\
782 \\
782 \\
787 \\
781\end{array}$ & $\begin{array}{l}296 \\
302 \\
304 \\
304 \\
312\end{array}$ & $\begin{array}{l}271 \\
293 \\
300 \\
298 \\
300\end{array}$ & $\begin{array}{l}286 \\
298 \\
302 \\
302 \\
303\end{array}$ & $\begin{array}{l}465 \\
482 \\
251 \\
263 \\
303\end{array}$ & $\begin{array}{l}423 \\
206 \\
143 \\
150 \\
241\end{array}$ & $\begin{array}{l}435 \\
310 \\
192 \\
204 \\
272\end{array}$ & $\begin{array}{l}293 \\
352 \\
301 \\
298 \\
292\end{array}$ & $\begin{array}{l}289 \\
289 \\
210 \\
227 \\
277\end{array}$ & $\begin{array}{l}291 \\
303 \\
268 \\
276 \\
281\end{array}$ \\
\hline $\begin{array}{l}21 \\
22 \\
23 \\
24 \\
25\end{array}$ & $\begin{array}{l}817 \\
814 \\
803 \\
799 \\
774\end{array}$ & $\begin{array}{l}782 \\
791 \\
790 \\
753 \\
742\end{array}$ & $\begin{array}{l}794 \\
800 \\
796 \\
770 \\
760\end{array}$ & $\begin{array}{l}336 \\
385 \\
394 \\
445 \\
491\end{array}$ & $\begin{array}{l}312 \\
336 \\
367 \\
381 \\
445\end{array}$ & $\begin{array}{l}326 \\
360 \\
379 \\
409 \\
458\end{array}$ & $\begin{array}{l}275 \\
300 \\
264 \\
265 \\
295\end{array}$ & $\begin{array}{l}239 \\
249 \\
183 \\
183 \\
268\end{array}$ & $\begin{array}{l}254 \\
272 \\
221 \\
213 \\
287\end{array}$ & $\begin{array}{l}282 \\
283 \\
286 \\
324 \\
318\end{array}$ & $\begin{array}{l}279 \\
278 \\
275 \\
279 \\
262\end{array}$ & $\begin{array}{l}281 \\
280 \\
281 \\
298 \\
288\end{array}$ \\
\hline $\begin{array}{l}26 \\
27 \\
28 \\
29 \\
30 \\
31\end{array}$ & $\begin{array}{l}757 \\
738 \\
976 \\
--- \\
--- \\
---\end{array}$ & $\begin{array}{l}735 \\
332 \\
530 \\
-\ldots \\
--- \\
---\end{array}$ & $\begin{array}{l}747 \\
535 \\
667 \\
--- \\
--- \\
---\end{array}$ & $\begin{array}{l}519 \\
532 \\
547 \\
547 \\
552 \\
567\end{array}$ & $\begin{array}{l}487 \\
499 \\
529 \\
524 \\
529 \\
547\end{array}$ & $\begin{array}{l}496 \\
516 \\
537 \\
539 \\
543 \\
555\end{array}$ & $\begin{array}{l}291 \\
295 \\
291 \\
--- \\
--- \\
---\end{array}$ & $\begin{array}{l}279 \\
288 \\
285 \\
--- \\
--- \\
---\end{array}$ & $\begin{array}{l}285 \\
292 \\
288 \\
--- \\
--- \\
---\end{array}$ & $\begin{array}{l}280 \\
228 \\
224 \\
269 \\
303 \\
292\end{array}$ & $\begin{array}{l}159 \\
172 \\
163 \\
206 \\
264 \\
249\end{array}$ & $\begin{array}{l}239 \\
192 \\
198 \\
245 \\
274 \\
267\end{array}$ \\
\hline \multirow[t]{2}{*}{ MONTH } & 976 & 332 & 730 & 567 & 217 & 411 & -- & - & - & 352 & 115 & 258 \\
\hline & \multicolumn{3}{|c|}{ JUNE } & \multicolumn{3}{|c|}{ JULY } & \multicolumn{3}{|c|}{ AUGUST } & \multicolumn{3}{|c|}{ SEPTEMBER } \\
\hline DAY & MAX & MIN & MEAN & MAX & MIN & MEAN & MAX & MIN & MEAN & MAX & MIN & MEAN \\
\hline $\begin{array}{l}1 \\
2 \\
3 \\
4 \\
5\end{array}$ & $\begin{array}{l}255 \\
234 \\
267 \\
210 \\
198\end{array}$ & $\begin{array}{l}129 \\
148 \\
210 \\
138 \\
152\end{array}$ & $\begin{array}{l}197 \\
203 \\
246 \\
175 \\
171\end{array}$ & $\begin{array}{l}222 \\
226 \\
236 \\
130 \\
144\end{array}$ & $\begin{array}{r}193 \\
219 \\
130 \\
84 \\
99\end{array}$ & $\begin{array}{l}205 \\
223 \\
208 \\
100 \\
110\end{array}$ & $\begin{array}{l}444 \\
583 \\
558 \\
394 \\
306\end{array}$ & $\begin{array}{l}400 \\
344 \\
301 \\
224 \\
277\end{array}$ & $\begin{array}{l}432 \\
407 \\
375 \\
317 \\
289\end{array}$ & $\begin{array}{l}447 \\
438 \\
444 \\
441 \\
437\end{array}$ & $\begin{array}{l}397 \\
405 \\
394 \\
391 \\
394\end{array}$ & $\begin{array}{l}415 \\
415 \\
414 \\
409 \\
412\end{array}$ \\
\hline $\begin{array}{r}6 \\
7 \\
8 \\
9 \\
10\end{array}$ & $\begin{array}{l}204 \\
153 \\
273 \\
297 \\
127\end{array}$ & $\begin{array}{l}126 \\
127 \\
143 \\
125 \\
116\end{array}$ & $\begin{array}{l}165 \\
142 \\
185 \\
210 \\
123\end{array}$ & $\begin{array}{l}244 \\
243 \\
239 \\
242 \\
246\end{array}$ & $\begin{array}{l}144 \\
233 \\
232 \\
239 \\
241\end{array}$ & $\begin{array}{l}222 \\
238 \\
235 \\
240 \\
243\end{array}$ & $\begin{array}{l}293 \\
312 \\
320 \\
317 \\
352\end{array}$ & $\begin{array}{l}270 \\
286 \\
302 \\
302 \\
312\end{array}$ & $\begin{array}{l}280 \\
302 \\
312 \\
311 \\
324\end{array}$ & $\begin{array}{l}444 \\
419 \\
414 \\
356 \\
363\end{array}$ & $\begin{array}{l}400 \\
303 \\
340 \\
315 \\
338\end{array}$ & $\begin{array}{l}412 \\
363 \\
374 \\
333 \\
348\end{array}$ \\
\hline $\begin{array}{l}11 \\
12 \\
13 \\
14 \\
15\end{array}$ & $\begin{array}{l}146 \\
220 \\
330 \\
403 \\
460\end{array}$ & $\begin{array}{l}118 \\
134 \\
220 \\
330 \\
403\end{array}$ & $\begin{array}{l}130 \\
173 \\
278 \\
369 \\
430\end{array}$ & $\begin{array}{l}244 \\
241 \\
252 \\
257 \\
273\end{array}$ & $\begin{array}{l}240 \\
237 \\
237 \\
248 \\
252\end{array}$ & $\begin{array}{l}242 \\
239 \\
246 \\
252 \\
260\end{array}$ & $\begin{array}{l}388 \\
443 \\
443 \\
466 \\
448\end{array}$ & $\begin{array}{l}350 \\
388 \\
419 \\
425 \\
420\end{array}$ & $\begin{array}{l}364 \\
411 \\
432 \\
440 \\
436\end{array}$ & $\begin{array}{l}404 \\
433 \\
414 \\
452 \\
451\end{array}$ & $\begin{array}{l}357 \\
384 \\
384 \\
397 \\
394\end{array}$ & $\begin{array}{l}370 \\
402 \\
397 \\
417 \\
418\end{array}$ \\
\hline $\begin{array}{l}16 \\
17 \\
18 \\
19 \\
20\end{array}$ & $\begin{array}{l}464 \\
353 \\
361 \\
345 \\
334\end{array}$ & $\begin{array}{l}345 \\
343 \\
345 \\
328 \\
325\end{array}$ & $\begin{array}{l}417 \\
347 \\
353 \\
334 \\
330\end{array}$ & $\begin{array}{l}263 \\
262 \\
265 \\
262 \\
336\end{array}$ & $\begin{array}{l}254 \\
253 \\
255 \\
258 \\
256\end{array}$ & $\begin{array}{l}257 \\
258 \\
261 \\
260 \\
273\end{array}$ & $\begin{array}{l}458 \\
446 \\
445 \\
443 \\
446\end{array}$ & $\begin{array}{l}416 \\
416 \\
408 \\
411 \\
414\end{array}$ & $\begin{array}{l}436 \\
430 \\
429 \\
427 \\
428\end{array}$ & $\begin{array}{l}423 \\
387 \\
266 \\
282 \\
291\end{array}$ & $\begin{array}{l}272 \\
242 \\
245 \\
243 \\
261\end{array}$ & $\begin{array}{l}356 \\
299 \\
259 \\
261 \\
279\end{array}$ \\
\hline $\begin{array}{l}21 \\
22 \\
23 \\
24 \\
25\end{array}$ & $\begin{array}{l}332 \\
329 \\
328 \\
301 \\
303\end{array}$ & $\begin{array}{l}322 \\
320 \\
161 \\
214 \\
270\end{array}$ & $\begin{array}{l}328 \\
326 \\
295 \\
280 \\
291\end{array}$ & $\begin{array}{l}269 \\
278 \\
260 \\
278 \\
262\end{array}$ & $\begin{array}{l}262 \\
227 \\
254 \\
250 \\
254\end{array}$ & $\begin{array}{l}265 \\
259 \\
257 \\
260 \\
259\end{array}$ & $\begin{array}{l}441 \\
438 \\
445 \\
438 \\
437\end{array}$ & $\begin{array}{l}407 \\
417 \\
412 \\
404 \\
405\end{array}$ & $\begin{array}{l}426 \\
424 \\
429 \\
426 \\
425\end{array}$ & $\begin{array}{l}324 \\
353 \\
368 \\
366 \\
381\end{array}$ & $\begin{array}{l}277 \\
316 \\
319 \\
328 \\
345\end{array}$ & $\begin{array}{l}304 \\
338 \\
350 \\
347 \\
364\end{array}$ \\
\hline $\begin{array}{l}26 \\
27 \\
28 \\
29 \\
30 \\
31\end{array}$ & $\begin{array}{l}341 \\
320 \\
327 \\
326 \\
214 \\
---\end{array}$ & $\begin{array}{l}301 \\
291 \\
317 \\
175 \\
147 \\
---\end{array}$ & $\begin{array}{l}321 \\
303 \\
322 \\
264 \\
171 \\
---\end{array}$ & $\begin{array}{l}302 \\
302 \\
353 \\
402 \\
461 \\
461\end{array}$ & $\begin{array}{l}243 \\
290 \\
296 \\
353 \\
402 \\
426\end{array}$ & $\begin{array}{l}276 \\
297 \\
314 \\
367 \\
425 \\
444\end{array}$ & $\begin{array}{l}433 \\
431 \\
426 \\
431 \\
425 \\
417\end{array}$ & $\begin{array}{l}401 \\
402 \\
394 \\
394 \\
396 \\
398\end{array}$ & $\begin{array}{l}422 \\
419 \\
411 \\
409 \\
411 \\
411\end{array}$ & $\begin{array}{l}380 \\
382 \\
365 \\
592 \\
529 \\
---\end{array}$ & $\begin{array}{l}337 \\
342 \\
194 \\
322 \\
370 \\
---\end{array}$ & $\begin{array}{l}358 \\
357 \\
308 \\
414 \\
436 \\
-\end{array}$ \\
\hline MONTH & 464 & 116 & 263 & 461 & 84 & 258 & 583 & 224 & 393 & 592 & 194 & 364 \\
\hline
\end{tabular}


ARKANSAS RIVER SUBBASIN

07178200 BIRD CRIR AT STATE BIGEMAY 266 NTAR CATOOSA, OKIAEOMA-CONTINUED

WATER TEMPERATURE, DEGREES CELSIUS, WATER YEAR OCTOBER 1994 TO SEPTEMBER 1995

OCTOBER NOVEMBER DECEMBER

\begin{tabular}{|c|c|c|c|c|c|c|c|c|c|c|c|c|}
\hline \multirow{2}{*}{ DAY } & & & & \\
\hline & MAX & MIN & MEAN & MAX & MIN & MEAN & MAX & MIN & MEAN & MAX & MIN & MEAN \\
\hline $\begin{array}{l}1 \\
2 \\
3 \\
4 \\
5\end{array}$ & $\begin{array}{l}23.5 \\
24.0 \\
23.5 \\
24.0 \\
24.0\end{array}$ & $\begin{array}{l}22.0 \\
22.5 \\
22.5 \\
22.5 \\
23.0\end{array}$ & $\begin{array}{l}23.0 \\
23.0 \\
23.0 \\
23.5 \\
23.0\end{array}$ & $\begin{array}{l}16.0 \\
16.0 \\
18.5 \\
18.5 \\
17.0\end{array}$ & $\begin{array}{l}15.0 \\
15.5 \\
16.0 \\
17.0 \\
12.5\end{array}$ & $\begin{array}{l}15.5 \\
15.5 \\
17.0 \\
18.0 \\
14.0\end{array}$ & $\begin{array}{l}11.5 \\
12.0 \\
12.5 \\
13.0 \\
13.0\end{array}$ & $\begin{array}{l}11.0 \\
11.0 \\
12.0 \\
12.5 \\
12.0\end{array}$ & $\begin{array}{l}11.5 \\
11.5 \\
12.0 \\
12.5 \\
12.5\end{array}$ & $\begin{array}{l}9.0 \\
7.0 \\
7.0 \\
6.0 \\
5.0\end{array}$ & $\begin{array}{l}6.5 \\
6.5 \\
6.0 \\
4.5 \\
3.5\end{array}$ & $\begin{array}{l}7.5 \\
6.5 \\
6.5 \\
5.0 \\
4.5\end{array}$ \\
\hline $\begin{array}{r}6 \\
7 \\
8 \\
9 \\
10\end{array}$ & $\begin{array}{l}23.5 \\
23.5 \\
19.5 \\
19.5 \\
19.0\end{array}$ & $\begin{array}{l}22.0 \\
19.0 \\
17.5 \\
18.5 \\
17.5\end{array}$ & $\begin{array}{l}22.5 \\
22.0 \\
18.5 \\
19.0 \\
18.0\end{array}$ & $\begin{array}{l}13.0 \\
14.0 \\
15.5 \\
16.0 \\
15.5\end{array}$ & $\begin{array}{l}12.0 \\
13.0 \\
14.0 \\
14.5 \\
14.0\end{array}$ & $\begin{array}{l}12.5 \\
13.5 \\
14.5 \\
15.5 \\
14.5\end{array}$ & $\begin{array}{r}12.0 \\
12.0 \\
10.5 \\
8.5 \\
7.5\end{array}$ & $\begin{array}{r}11.5 \\
10.5 \\
8.5 \\
7.5 \\
6.5\end{array}$ & $\begin{array}{r}12.0 \\
11.5 \\
10.0 \\
8.0 \\
7.0\end{array}$ & $\begin{array}{l}5.0 \\
5.0 \\
5.0 \\
6.0 \\
7.5\end{array}$ & $\begin{array}{l}4.5 \\
4.0 \\
4.5 \\
5.0 \\
6.0\end{array}$ & $\begin{array}{l}4.5 \\
4.5 \\
5.0 \\
5.5 \\
6.5\end{array}$ \\
\hline $\begin{array}{l}11 \\
12 \\
13 \\
14 \\
15\end{array}$ & $\begin{array}{l}18.5 \\
19.0 \\
19.0 \\
19.0 \\
19.0\end{array}$ & $\begin{array}{l}17.5 \\
17.0 \\
18.0 \\
18.0 \\
18.0\end{array}$ & $\begin{array}{l}18.0 \\
18.0 \\
18.5 \\
18.5 \\
18.5\end{array}$ & $\begin{array}{l}14.0 \\
13.5 \\
14.5 \\
15.0 \\
14.5\end{array}$ & $\begin{array}{l}13.0 \\
12.5 \\
13.5 \\
14.0 \\
14.0\end{array}$ & $\begin{array}{l}13.5 \\
13.0 \\
13.5 \\
14.5 \\
14.5\end{array}$ & $\begin{array}{l}7.5 \\
7.5 \\
8.0 \\
8.5 \\
8.5\end{array}$ & $\begin{array}{l}6.5 \\
6.5 \\
7.0 \\
8.0 \\
8.0\end{array}$ & $\begin{array}{l}7.0 \\
7.0 \\
7.5 \\
8.0 \\
8.5\end{array}$ & $\begin{array}{l}8.0 \\
8.5 \\
9.0 \\
8.5 \\
8.5\end{array}$ & $\begin{array}{l}7.5 \\
8.0 \\
8.0 \\
8.0 \\
7.5\end{array}$ & $\begin{array}{l}7.5 \\
8.5 \\
8.5 \\
8.5 \\
8.0\end{array}$ \\
\hline $\begin{array}{l}16 \\
17 \\
18 \\
19 \\
20\end{array}$ & $\begin{array}{l}19.0 \\
19.5 \\
20.0 \\
20.0 \\
20.0\end{array}$ & $\begin{array}{l}18.5 \\
19.0 \\
19.5 \\
19.0 \\
19.0\end{array}$ & $\begin{array}{l}18.5 \\
19.0 \\
19.5 \\
19.5 \\
19.5\end{array}$ & $\begin{array}{l}14.0 \\
13.5 \\
14.0 \\
14.0 \\
12.5\end{array}$ & $\begin{array}{l}13.0 \\
12.5 \\
12.5 \\
12.5 \\
11.5\end{array}$ & $\begin{array}{l}13.5 \\
13.0 \\
13.0 \\
13.5 \\
12.0\end{array}$ & $\begin{array}{l}8.5 \\
8.5 \\
8.5 \\
8.5 \\
9.0\end{array}$ & $\begin{array}{l}7.5 \\
7.5 \\
7.5 \\
7.5 \\
8.0\end{array}$ & $\begin{array}{l}8.0 \\
8.0 \\
8.0 \\
8.0 \\
8.5\end{array}$ & $\begin{array}{r}9.0 \\
10.0 \\
9.5 \\
8.5 \\
8.0\end{array}$ & $\begin{array}{l}8.0 \\
9.0 \\
8.0 \\
6.0 \\
6.0\end{array}$ & $\begin{array}{l}8.5 \\
9.5 \\
9.0 \\
7.0 \\
7.0\end{array}$ \\
\hline $\begin{array}{l}21 \\
22 \\
23 \\
24 \\
25\end{array}$ & $\begin{array}{l}21.0 \\
20.5 \\
20.5 \\
20.0 \\
19.0\end{array}$ & $\begin{array}{l}19.5 \\
19.5 \\
19.0 \\
19.0 \\
17.5\end{array}$ & $\begin{array}{l}20.0 \\
20.0 \\
19.5 \\
19.0 \\
18.5\end{array}$ & $\begin{array}{l}12.0 \\
12.0 \\
11.0 \\
11.0 \\
12.0\end{array}$ & $\begin{array}{r}11.0 \\
10.0 \\
9.5 \\
10.5 \\
11.0\end{array}$ & $\begin{array}{l}11.5 \\
11.0 \\
10.5 \\
11.0 \\
11.5\end{array}$ & $\begin{array}{l}9.0 \\
9.0 \\
9.0 \\
8.0 \\
8.0\end{array}$ & $\begin{array}{l}8.5 \\
8.5 \\
8.0 \\
7.5 \\
7.0\end{array}$ & $\begin{array}{l}9.0 \\
8.5 \\
8.5 \\
7.5 \\
7.5\end{array}$ & $\begin{array}{l}8.0 \\
7.5 \\
7.5 \\
7.5 \\
8.0\end{array}$ & $\begin{array}{l}7.0 \\
6.5 \\
6.5 \\
6.5 \\
7.5\end{array}$ & $\begin{array}{l}7.0 \\
7.0 \\
7.0 \\
7.0 \\
7.5\end{array}$ \\
\hline $\begin{array}{l}26 \\
27 \\
28 \\
29 \\
30 \\
31\end{array}$ & $\begin{array}{l}17.5 \\
16.5 \\
16.5 \\
16.8 \\
17.2 \\
17.5\end{array}$ & $\begin{array}{l}16.5 \\
15.5 \\
15.7 \\
15.8 \\
16.5 \\
16.0\end{array}$ & $\begin{array}{l}17.0 \\
16.0 \\
16.1 \\
16.4 \\
16.9 \\
17.0\end{array}$ & $\begin{array}{l}13.0 \\
13.5 \\
13.0 \\
12.5 \\
12.0 \\
-\end{array}$ & $\begin{array}{l}12.0 \\
13.0 \\
12.5 \\
12.0 \\
11.5 \\
---\end{array}$ & $\begin{array}{l}12.5 \\
13.0 \\
12.5 \\
12.0 \\
11.5 \\
-\end{array}$ & $\begin{array}{l}7.5 \\
8.0 \\
8.5 \\
9.0 \\
9.0 \\
9.0\end{array}$ & $\begin{array}{l}7.0 \\
7.0 \\
7.5 \\
8.0 \\
8.5 \\
8.5\end{array}$ & $\begin{array}{l}7.0 \\
7.5 \\
8.0 \\
8.5 \\
8.5 \\
9.0\end{array}$ & $\begin{array}{r}8.0 \\
10.0 \\
10.0 \\
9.0 \\
8.5 \\
9.0\end{array}$ & $\begin{array}{l}7.5 \\
8.0 \\
9.0 \\
8.0 \\
8.0 \\
7.5\end{array}$ & $\begin{array}{l}8.0 \\
9.0 \\
9.5 \\
8.5 \\
8.0 \\
8.0\end{array}$ \\
\hline \multirow[t]{2}{*}{ MONTH } & 24.0 & 15.5 & 19.3 & 18.5 & 9.5 & 13.4 & 13.0 & 6.5 & 8.9 & 10.0 & 3.5 & 7.2 \\
\hline & \multicolumn{3}{|c|}{ FEBRUARY } & \multicolumn{3}{|c|}{ MARCH } & \multicolumn{3}{|c|}{ APRIL } & \multicolumn{3}{|c|}{ MAY } \\
\hline DAY & MAX & MIN & MEAN & MAX & MIN & MEAN & MAX & MIN & MEAN & MAX & MIN & MEAN \\
\hline $\begin{array}{l}1 \\
2 \\
3 \\
4 \\
5\end{array}$ & $\begin{array}{r}9.5 \\
10.5 \\
10.5 \\
10.0 \\
10.0\end{array}$ & $\begin{array}{r}8.5 \\
9.0 \\
10.0 \\
9.0 \\
9.0\end{array}$ & $\begin{array}{r}8.5 \\
9.5 \\
10.0 \\
9.5 \\
9.5\end{array}$ & $\begin{array}{l}9.5 \\
7.0 \\
6.5 \\
6.5 \\
7.0\end{array}$ & $\begin{array}{l}7.0 \\
6.0 \\
5.5 \\
6.0 \\
6.5\end{array}$ & $\begin{array}{l}8.0 \\
6.5 \\
6.0 \\
6.5 \\
6.5\end{array}$ & $\begin{array}{l}15.5 \\
16.5 \\
16.5 \\
16.5 \\
17.0\end{array}$ & $\begin{array}{l}14.0 \\
14.5 \\
15.5 \\
15.0 \\
15.5\end{array}$ & $\begin{array}{l}14.5 \\
15.5 \\
16.0 \\
16.0 \\
16.0\end{array}$ & $\begin{array}{l}15.0 \\
15.0 \\
14.0 \\
14.0 \\
15.0\end{array}$ & $\begin{array}{l}15.0 \\
14.0 \\
14.0 \\
13.5 \\
14.0\end{array}$ & $\begin{array}{l}15.0 \\
14.5 \\
14.0 \\
14.0 \\
14.5\end{array}$ \\
\hline $\begin{array}{r}6 \\
7 \\
8 \\
9 \\
10\end{array}$ & $\begin{array}{l}9.5 \\
9.5 \\
9.0 \\
9.0 \\
9.5\end{array}$ & $\begin{array}{l}9.0 \\
8.5 \\
8.0 \\
8.0 \\
8.5\end{array}$ & $\begin{array}{l}9.5 \\
9.0 \\
8.5 \\
8.5 \\
9.0\end{array}$ & $\begin{array}{l}7.5 \\
7.5 \\
6.0 \\
6.5 \\
8.5\end{array}$ & $\begin{array}{l}6.5 \\
5.5 \\
5.0 \\
5.5 \\
6.5\end{array}$ & $\begin{array}{l}7.0 \\
6.5 \\
5.5 \\
6.0 \\
7.5\end{array}$ & $\begin{array}{l}17.5 \\
18.5 \\
20.0 \\
20.5 \\
20.5\end{array}$ & $\begin{array}{l}16.0 \\
17.0 \\
18.0 \\
19.5 \\
17.0\end{array}$ & $\begin{array}{l}17.0 \\
17.5 \\
19.0 \\
20.0 \\
19.5\end{array}$ & $\begin{array}{l}15.0 \\
17.0 \\
17.0 \\
17.5 \\
18.0\end{array}$ & $\begin{array}{l}14.0 \\
15.0 \\
16.0 \\
16.5 \\
17.5\end{array}$ & $\begin{array}{l}14.5 \\
15.5 \\
16.5 \\
17.0 \\
18.0\end{array}$ \\
\hline $\begin{array}{l}11 \\
12 \\
13 \\
14 \\
15\end{array}$ & $\begin{array}{l}9.0 \\
8.0 \\
7.0 \\
7.0 \\
7.0\end{array}$ & $\begin{array}{l}7.5 \\
6.0 \\
6.0 \\
6.0 \\
6.5\end{array}$ & $\begin{array}{l}8.5 \\
7.0 \\
6.5 \\
6.5 \\
6.5\end{array}$ & $\begin{array}{l}10.0 \\
11.0 \\
13.0 \\
12.5 \\
14.0\end{array}$ & $\begin{array}{r}7.5 \\
9.5 \\
11.0 \\
12.0 \\
12.5\end{array}$ & $\begin{array}{r}8.5 \\
10.5 \\
11.5 \\
12.5 \\
13.5\end{array}$ & $\begin{array}{l}17.0 \\
14.5 \\
15.0 \\
15.5 \\
17.0\end{array}$ & $\begin{array}{l}14.5 \\
14.0 \\
13.5 \\
14.0 \\
14.5\end{array}$ & $\begin{array}{l}15.5 \\
14.5 \\
14.0 \\
14.5 \\
15.5\end{array}$ & $\begin{array}{l}18.0 \\
17.0 \\
17.5 \\
17.5 \\
18.0\end{array}$ & $\begin{array}{l}16.0 \\
16.0 \\
16.0 \\
16.5 \\
16.5\end{array}$ & $\begin{array}{l}17.0 \\
16.5 \\
17.0 \\
17.0 \\
17.0\end{array}$ \\
\hline $\begin{array}{l}16 \\
17 \\
18 \\
19 \\
20\end{array}$ & $\begin{array}{l}7.0 \\
7.0 \\
7.5 \\
8.5 \\
9.5\end{array}$ & $\begin{array}{l}6.0 \\
6.0 \\
7.0 \\
7.5 \\
8.0\end{array}$ & $\begin{array}{l}6.5 \\
6.5 \\
7.5 \\
8.0 \\
9.0\end{array}$ & $\begin{array}{l}14.0 \\
11.0 \\
11.0 \\
12.0 \\
12.5\end{array}$ & $\begin{array}{l}10.5 \\
10.0 \\
10.0 \\
10.5 \\
11.5\end{array}$ & $\begin{array}{l}11.5 \\
10.5 \\
10.5 \\
11.0 \\
12.0\end{array}$ & $\begin{array}{l}18.0 \\
18.5 \\
17.5 \\
17.0 \\
17.0\end{array}$ & $\begin{array}{l}16.5 \\
17.5 \\
16.0 \\
16.0 \\
16.0\end{array}$ & $\begin{array}{l}17.0 \\
18.0 \\
16.5 \\
16.5 \\
16.0\end{array}$ & $\begin{array}{l}19.0 \\
21.5 \\
22.0 \\
22.0 \\
20.5\end{array}$ & $\begin{array}{l}17.5 \\
18.5 \\
19.0 \\
20.5 \\
19.0\end{array}$ & $\begin{array}{l}18.0 \\
19.5 \\
20.5 \\
21.0 \\
19.5\end{array}$ \\
\hline $\begin{array}{l}21 \\
22 \\
23 \\
24 \\
25\end{array}$ & $\begin{array}{l}10.0 \\
13.0 \\
12.0 \\
12.5 \\
12.5\end{array}$ & $\begin{array}{r}9.0 \\
9.5 \\
10.5 \\
11.5 \\
11.5\end{array}$ & $\begin{array}{r}9.5 \\
10.5 \\
11.5 \\
12.0 \\
12.0\end{array}$ & $\begin{array}{l}13.0 \\
16.0 \\
16.5 \\
16.5 \\
16.5\end{array}$ & $\begin{array}{l}12.0 \\
13.0 \\
15.0 \\
15.0 \\
15.5\end{array}$ & $\begin{array}{l}12.5 \\
14.5 \\
16.0 \\
15.5 \\
16.0\end{array}$ & $\begin{array}{l}17.0 \\
16.0 \\
13.0 \\
13.0 \\
14.5\end{array}$ & $\begin{array}{l}16.0 \\
13.0 \\
12.0 \\
12.0 \\
13.0\end{array}$ & $\begin{array}{l}16.5 \\
14.5 \\
12.5 \\
12.5 \\
13.5\end{array}$ & $\begin{array}{l}19.5 \\
19.0 \\
18.5 \\
18.5 \\
19.0\end{array}$ & $\begin{array}{l}18.5 \\
17.5 \\
17.5 \\
17.5 \\
17.0\end{array}$ & $\begin{array}{l}19.0 \\
18.5 \\
18.0 \\
18.0 \\
17.5\end{array}$ \\
\hline $\begin{array}{l}26 \\
27 \\
28 \\
29 \\
30 \\
31\end{array}$ & $\begin{array}{l}13.0 \\
14.0 \\
12.5 \\
-- \\
--\end{array}$ & $\begin{array}{r}12.0 \\
12.5 \\
9.5 \\
- \\
- \\
--\end{array}$ & $\begin{array}{l}13.0 \\
13.0 \\
11.0 \\
--- \\
--\end{array}$ & $\begin{array}{l}16.5 \\
16.5 \\
16.5 \\
15.0 \\
14.5 \\
14.5\end{array}$ & $\begin{array}{l}16.0 \\
15.5 \\
15.0 \\
14.0 \\
13.5 \\
13.5\end{array}$ & $\begin{array}{l}16.0 \\
16.0 \\
15.0 \\
14.5 \\
14.0 \\
14.0\end{array}$ & $\begin{array}{l}15.0 \\
15.0 \\
--- \\
-- \\
-- \\
--\end{array}$ & $\begin{array}{c}14.5 \\
14.5 \\
--- \\
--- \\
---\end{array}$ & $\begin{array}{c}15.0 \\
15.0 \\
-\square \\
-\square \\
--\end{array}$ & $\begin{array}{l}17.5 \\
19.0 \\
20.5 \\
20.5 \\
19.0 \\
18.5\end{array}$ & $\begin{array}{l}16.5 \\
17.5 \\
19.0 \\
19.0 \\
17.5 \\
17.0\end{array}$ & $\begin{array}{l}17.0 \\
18.5 \\
20.0 \\
19.5 \\
18.0 \\
18.0\end{array}$ \\
\hline MONTH & 14.0 & 6.0 & 9.2 & 16.5 & 5.0 & 11.0 & $-\infty$ & -- & -- & 22.0 & 13.5 & 17.4 \\
\hline
\end{tabular}


ARKANSAS RTVER SUBBASIN

07178200 BIRD CREEK AT STATE BIGEMAY 266 NEAR CATOOSA, OKLAHOMA--CONTINOED

WATER TEMPERATURE, DEGREES CELSIUS, WATER YEAR OCTOBER 1994 TO SEPTEMBER 1995

$$
\text { JUNE JULY }
$$

WS, WATER YEAR OCTOBER 199
AUGUST

\begin{tabular}{|c|c|c|c|c|c|c|c|c|c|c|c|c|}
\hline \multirow[b]{2}{*}{ DAY } & \multicolumn{3}{|c|}{ JUNE } & \multicolumn{3}{|c|}{ JULY } & \multicolumn{3}{|c|}{ AUGUST } & \multicolumn{3}{|c|}{ SEPTEMBER } \\
\hline & MAX & MIN & MEAN & MAX & MIN & MEAN & $\operatorname{MAX}$ & MIN & MEAN & MAX & MIN & MEAN \\
\hline $\begin{array}{l}1 \\
2 \\
3 \\
4 \\
5\end{array}$ & $\begin{array}{l}18.5 \\
20.0 \\
20.5 \\
21.5 \\
22.0\end{array}$ & $\begin{array}{l}17.5 \\
18.0 \\
18.5 \\
19.0 \\
20.5\end{array}$ & $\begin{array}{l}18.0 \\
19.0 \\
19.5 \\
20.5 \\
21.5\end{array}$ & $\begin{array}{l}21.5 \\
21.0 \\
21.5 \\
22.0 \\
23.5\end{array}$ & $\begin{array}{l}20.5 \\
20.0 \\
20.5 \\
20.5 \\
22.0\end{array}$ & $\begin{array}{l}21.0 \\
20.5 \\
20.5 \\
21.5 \\
23.0\end{array}$ & $\begin{array}{l}29.0 \\
28.0 \\
27.5 \\
27.0 \\
26.5\end{array}$ & $\begin{array}{l}27.5 \\
25.0 \\
26.5 \\
26.0 \\
25.5\end{array}$ & $\begin{array}{l}28.5 \\
27.0 \\
27.0 \\
26.5 \\
26.0\end{array}$ & $\begin{array}{l}29.5 \\
29.0 \\
29.0 \\
29.0 \\
29.0\end{array}$ & $\begin{array}{l}28.5 \\
28.5 \\
28.5 \\
28.0 \\
28.0\end{array}$ & $\begin{array}{l}29.0 \\
29.0 \\
29.0 \\
28.5 \\
28.5\end{array}$ \\
\hline $\begin{array}{r}6 \\
7 \\
8 \\
9 \\
10\end{array}$ & $\begin{array}{l}22.0 \\
22.0 \\
24.0 \\
23.5 \\
20.5\end{array}$ & $\begin{array}{l}21.0 \\
20.5 \\
22.0 \\
20.0 \\
20.0\end{array}$ & $\begin{array}{l}21.5 \\
21.0 \\
23.0 \\
21.5 \\
20.5\end{array}$ & $\begin{array}{l}23.5 \\
22.5 \\
22.0 \\
22.5 \\
22.5\end{array}$ & $\begin{array}{l}22.0 \\
21.5 \\
21.5 \\
21.5 \\
21.5\end{array}$ & $\begin{array}{l}22.5 \\
22.0 \\
22.0 \\
22.0 \\
22.0\end{array}$ & $\begin{array}{l}26.0 \\
27.0 \\
27.0 \\
27.0 \\
27.5\end{array}$ & $\begin{array}{l}24.5 \\
25.0 \\
25.5 \\
25.5 \\
25.5\end{array}$ & $\begin{array}{l}25.5 \\
25.5 \\
26.0 \\
26.0 \\
26.0\end{array}$ & $\begin{array}{l}28.5 \\
28.0 \\
26.0 \\
25.0 \\
24.5\end{array}$ & $\begin{array}{l}27.5 \\
25.0 \\
24.5 \\
24.0 \\
22.5\end{array}$ & $\begin{array}{l}28.0 \\
25.5 \\
25.5 \\
24.5 \\
23.5\end{array}$ \\
\hline $\begin{array}{l}11 \\
12 \\
13 \\
14 \\
15\end{array}$ & $\begin{array}{l}21.0 \\
22.0 \\
22.5 \\
24.0 \\
25.0\end{array}$ & $\begin{array}{l}20.5 \\
20.5 \\
21.0 \\
22.0 \\
23.0\end{array}$ & $\begin{array}{l}20.5 \\
21.0 \\
21.5 \\
23.0 \\
24.0\end{array}$ & $\begin{array}{l}22.5 \\
22.5 \\
22.5 \\
22.5 \\
24.0\end{array}$ & $\begin{array}{l}21.5 \\
22.0 \\
22.0 \\
22.0 \\
22.5\end{array}$ & $\begin{array}{l}22.0 \\
22.0 \\
22.5 \\
22.5 \\
23.0\end{array}$ & $\begin{array}{l}28.0 \\
29.5 \\
30.0 \\
30.0 \\
30.0\end{array}$ & $\begin{array}{l}26.0 \\
27.0 \\
28.5 \\
28.5 \\
29.0\end{array}$ & $\begin{array}{l}27.0 \\
28.0 \\
29.0 \\
29.5 \\
29.5\end{array}$ & $\begin{array}{l}24.5 \\
24.0 \\
25.5 \\
26.0 \\
26.0\end{array}$ & $\begin{array}{l}23.0 \\
23.0 \\
23.5 \\
24.5 \\
25.0\end{array}$ & $\begin{array}{l}23.5 \\
23.5 \\
24.5 \\
25.0 \\
25.5\end{array}$ \\
\hline $\begin{array}{l}16 \\
17 \\
18 \\
19 \\
20\end{array}$ & $\begin{array}{l}25.5 \\
23.0 \\
22.5 \\
22.0 \\
22.5\end{array}$ & $\begin{array}{l}21.5 \\
21.5 \\
21.5 \\
21.0 \\
21.0\end{array}$ & $\begin{array}{l}24.0 \\
22.0 \\
22.0 \\
21.5 \\
21.5\end{array}$ & $\begin{array}{l}23.5 \\
23.5 \\
24.0 \\
23.0 \\
24.0\end{array}$ & $\begin{array}{l}22.5 \\
23.0 \\
22.5 \\
22.5 \\
22.5\end{array}$ & $\begin{array}{l}23.0 \\
23.0 \\
23.0 \\
23.0 \\
23.0\end{array}$ & $\begin{array}{l}30.0 \\
30.0 \\
30.0 \\
30.0 \\
30.5\end{array}$ & $\begin{array}{l}28.5 \\
29.0 \\
29.0 \\
29.0 \\
29.5\end{array}$ & $\begin{array}{l}29.5 \\
29.5 \\
29.5 \\
30.0 \\
30.0\end{array}$ & $\begin{array}{l}25.0 \\
24.5 \\
24.0 \\
24.0 \\
24.0\end{array}$ & $\begin{array}{l}23.5 \\
23.5 \\
23.5 \\
23.5 \\
22.0\end{array}$ & $\begin{array}{l}24.5 \\
24.0 \\
23.5 \\
23.5 \\
22.5\end{array}$ \\
\hline $\begin{array}{l}21 \\
22 \\
23 \\
24 \\
25\end{array}$ & $\begin{array}{l}22.5 \\
23.0 \\
23.0 \\
22.5 \\
22.5\end{array}$ & $\begin{array}{l}21.0 \\
21.5 \\
21.5 \\
21.5 \\
21.5\end{array}$ & $\begin{array}{l}22.0 \\
22.0 \\
22.0 \\
22.0 \\
22.0\end{array}$ & $\begin{array}{l}24.0 \\
24.5 \\
24.0 \\
24.0 \\
24.0\end{array}$ & $\begin{array}{l}22.5 \\
23.0 \\
23.0 \\
23.0 \\
23.0\end{array}$ & $\begin{array}{l}23.0 \\
23.5 \\
23.5 \\
23.5 \\
23.5\end{array}$ & $\begin{array}{l}30.5 \\
30.0 \\
30.0 \\
29.5 \\
29.0\end{array}$ & $\begin{array}{l}29.0 \\
29.0 \\
29.0 \\
28.0 \\
27.5\end{array}$ & $\begin{array}{l}29.5 \\
29.5 \\
29.5 \\
28.5 \\
28.5\end{array}$ & $\begin{array}{l}22.0 \\
19.5 \\
19.0 \\
19.0 \\
18.5\end{array}$ & $\begin{array}{l}19.5 \\
18.5 \\
17.5 \\
17.0 \\
17.5\end{array}$ & $\begin{array}{l}21.0 \\
19.0 \\
18.0 \\
18.0 \\
18.0\end{array}$ \\
\hline $\begin{array}{l}26 \\
27 \\
28 \\
29 \\
30 \\
31\end{array}$ & $\begin{array}{l}22.5 \\
22.5 \\
23.0 \\
22.0 \\
22.0 \\
-\end{array}$ & $\begin{array}{l}21.5 \\
21.0 \\
21.5 \\
20.5 \\
21.0 \\
-\end{array}$ & $\begin{array}{l}22.0 \\
21.5 \\
22.0 \\
21.5 \\
22.0 \\
-\end{array}$ & $\begin{array}{l}25.0 \\
25.5 \\
27.0 \\
28.0 \\
29.0 \\
29.0\end{array}$ & $\begin{array}{l}23.0 \\
24.0 \\
24.0 \\
25.5 \\
27.0 \\
28.5\end{array}$ & $\begin{array}{l}24.0 \\
24.5 \\
25.0 \\
26.5 \\
28.0 \\
29.0\end{array}$ & $\begin{array}{l}29.0 \\
29.0 \\
29.0 \\
29.5 \\
29.5 \\
29.5\end{array}$ & $\begin{array}{l}28.0 \\
28.0 \\
28.5 \\
28.5 \\
28.5 \\
28.5\end{array}$ & $\begin{array}{l}28.5 \\
28.5 \\
29.0 \\
29.0 \\
29.0 \\
29.0\end{array}$ & $\begin{array}{l}19.0 \\
20.0 \\
21.0 \\
22.5 \\
23.0 \\
---\end{array}$ & $\begin{array}{l}17.5 \\
18.0 \\
19.5 \\
20.5 \\
22.0\end{array}$ & $\begin{array}{l}18.0 \\
19.0 \\
20.0 \\
21.5 \\
22.0 \\
\end{array}$ \\
\hline $\mathrm{TH}$ & 25.5 & 17.5 & 21.5 & 29.0 & 20.0 & 23.2 & 30.5 & 24.5 & 28.2 & 29.5 & 17.0 & 23.5 \\
\hline
\end{tabular}

\begin{tabular}{|c|c|c|c|c|c|c|c|c|c|c|c|c|}
\hline \multirow[t]{2}{*}{ MONTH } & \multicolumn{2}{|c|}{25.5} & 17.5 & 29.0 & 20. & 23.2 & 30.5 & 24.5 & 28.2 & 29.5 & 17.0 & 23 \\
\hline & \multicolumn{3}{|c|}{$\begin{array}{l}\text { PH, WATER, W } \\
\text { OCTOBER }\end{array}$} & FIELD, & \multicolumn{2}{|c|}{$\begin{array}{l}\text { STANDARD } \\
\text { NOVEMBER }\end{array}$} & WATER YEAR & \multicolumn{2}{|c|}{$\begin{array}{l}\text { OCTOBER } \\
\text { DECEMBER }\end{array}$} & \multicolumn{3}{|c|}{$\begin{array}{r}\text { SEPTEMBER } 1995 \\
\text { JANUARY }\end{array}$} \\
\hline DAY & MAX & MIN & MEDIAN & MAX & MIN & MEDIAN & MAX & MIN & MEDIAN & MAX & MIN & MEDIAN \\
\hline $\begin{array}{l}1 \\
2 \\
3 \\
4 \\
5\end{array}$ & $\begin{array}{l}7.9 \\
8.0 \\
7.8 \\
7.9 \\
7.8\end{array}$ & $\begin{array}{l}7.4 \\
7.4 \\
7.4 \\
7.4 \\
7.5\end{array}$ & $\begin{array}{l}7.7 \\
7.7 \\
7.7 \\
7.7 \\
7.6\end{array}$ & $\begin{array}{l}7.5 \\
7.5 \\
7.5 \\
7.6 \\
7.5\end{array}$ & $\begin{array}{l}7.3 \\
7.3 \\
7.2 \\
7.2 \\
7.2\end{array}$ & $\begin{array}{l}7.4 \\
7.3 \\
7.4 \\
7.5 \\
7.3\end{array}$ & $\begin{array}{l}7.7 \\
7.6 \\
7.5 \\
7.5 \\
7.6\end{array}$ & $\begin{array}{l}7.5 \\
7.5 \\
7.5 \\
7.5 \\
7.5\end{array}$ & $\begin{array}{l}7.6 \\
7.5 \\
7.5 \\
7.5 \\
7.5\end{array}$ & $\begin{array}{l}8.0 \\
8.0 \\
7.9 \\
7.9 \\
7.9\end{array}$ & $\begin{array}{l}7.8 \\
7.8 \\
7.8 \\
7.8 \\
7.8\end{array}$ & $\begin{array}{l}7.9 \\
7.9 \\
7.9 \\
7.8 \\
7.9\end{array}$ \\
\hline $\begin{array}{r}6 \\
7 \\
8 \\
9 \\
10\end{array}$ & $\begin{array}{l}7.7 \\
7.8 \\
7.6 \\
7.3 \\
7.3\end{array}$ & $\begin{array}{l}7.4 \\
7.4 \\
7.2 \\
7.2 \\
7.2\end{array}$ & $\begin{array}{l}7.6 \\
7.5 \\
7.3 \\
7.3 \\
7.3\end{array}$ & $\begin{array}{l}7.4 \\
7.6 \\
7.7 \\
7.8 \\
7.6\end{array}$ & $\begin{array}{l}7.2 \\
7.4 \\
7.6 \\
7.4 \\
7.4\end{array}$ & $\begin{array}{l}7.3 \\
7.6 \\
7.7 \\
7.7 \\
7.5\end{array}$ & $\begin{array}{l}7.6 \\
7.7 \\
7.7 \\
7.7 \\
7.7\end{array}$ & $\begin{array}{l}7.6 \\
7.6 \\
7.6 \\
7.6 \\
7.6\end{array}$ & $\begin{array}{l}7.6 \\
7.6 \\
7.7 \\
7.6 \\
7.6\end{array}$ & $\begin{array}{l}7.9 \\
8.0 \\
8.0 \\
8.0 \\
8.0\end{array}$ & $\begin{array}{l}7.9 \\
7.9 \\
7.9 \\
7.9 \\
7.9\end{array}$ & $\begin{array}{l}7.9 \\
7.9 \\
8.0 \\
7.9 \\
7.9\end{array}$ \\
\hline $\begin{array}{l}11 \\
12 \\
13 \\
14 \\
15\end{array}$ & $\begin{array}{l}7.4 \\
7.5 \\
7.5 \\
7.5 \\
7.6\end{array}$ & $\begin{array}{l}7.2 \\
7.2 \\
7.3 \\
7.2 \\
7.3\end{array}$ & $\begin{array}{l}7.3 \\
7.4 \\
7.4 \\
7.4 \\
7.4\end{array}$ & $\begin{array}{l}7.8 \\
7.8 \\
7.8 \\
7.8 \\
7.8\end{array}$ & $\begin{array}{l}7.6 \\
7.7 \\
7.7 \\
7.7 \\
7.5\end{array}$ & $\begin{array}{l}7.7 \\
7.8 \\
7.8 \\
7.7 \\
7.6\end{array}$ & $\begin{array}{l}7.8 \\
7.8 \\
7.8 \\
7.8 \\
7.7\end{array}$ & $\begin{array}{l}7.6 \\
7.7 \\
7.7 \\
7.7 \\
7.6\end{array}$ & $\begin{array}{l}7.7 \\
7.8 \\
7.8 \\
7.7 \\
7.7\end{array}$ & $\begin{array}{l}8.0 \\
7.8 \\
7.7 \\
7.8 \\
7.8\end{array}$ & $\begin{array}{l}7.7 \\
7.7 \\
7.6 \\
7.7 \\
7.7\end{array}$ & $\begin{array}{l}7.9 \\
7.7 \\
7.7 \\
7.7 \\
7.7\end{array}$ \\
\hline $\begin{array}{l}16 \\
17 \\
18 \\
19 \\
20\end{array}$ & $\begin{array}{l}7.6 \\
7.5 \\
7.6 \\
7.5 \\
7.5\end{array}$ & $\begin{array}{l}7.3 \\
7.3 \\
7.3 \\
7.3 \\
7.2\end{array}$ & $\begin{array}{l}7.5 \\
7.4 \\
7.4 \\
7.4 \\
7.4\end{array}$ & $\begin{array}{l}7.6 \\
7.7 \\
7.8 \\
7.7 \\
7.7\end{array}$ & $\begin{array}{l}7.4 \\
7.5 \\
7.5 \\
7.5 \\
7.3\end{array}$ & $\begin{array}{l}7.6 \\
7.6 \\
7.7 \\
7.6 \\
7.5\end{array}$ & $\begin{array}{l}7.9 \\
7.8 \\
7.8 \\
7.9 \\
7.9\end{array}$ & $\begin{array}{l}7.7 \\
7.7 \\
7.7 \\
7.7 \\
7.7\end{array}$ & $\begin{array}{l}7.8 \\
7.7 \\
7.8 \\
7.7 \\
7.7\end{array}$ & $\begin{array}{l}7.8 \\
7.8 \\
7.8 \\
7.8 \\
7.7\end{array}$ & $\begin{array}{l}7.7 \\
7.7 \\
7.7 \\
7.7 \\
7.7\end{array}$ & $\begin{array}{l}7.7 \\
7.8 \\
7.7 \\
7.7 \\
7.7\end{array}$ \\
\hline $\begin{array}{l}21 \\
22 \\
23 \\
24 \\
25\end{array}$ & $\begin{array}{l}7.4 \\
7.4 \\
7.4 \\
7.4 \\
7.5\end{array}$ & $\begin{array}{l}7.3 \\
7.3 \\
7.2 \\
7.2 \\
7.2\end{array}$ & $\begin{array}{l}7.3 \\
7.4 \\
7.3 \\
7.3 \\
7.3\end{array}$ & $\begin{array}{l}7.7 \\
7.6 \\
7.6 \\
7.6 \\
7.6\end{array}$ & $\begin{array}{l}7.2 \\
7.3 \\
7.4 \\
7.5 \\
7.5\end{array}$ & $\begin{array}{l}7.5 \\
7.4 \\
7.5 \\
7.5 \\
7.6\end{array}$ & $\begin{array}{l}7.9 \\
7.9 \\
7.9 \\
7.9 \\
7.9\end{array}$ & $\begin{array}{l}7.8 \\
7.9 \\
7.9 \\
7.9 \\
7.9\end{array}$ & $\begin{array}{l}7.9 \\
7.9 \\
7.9 \\
7.9 \\
7.9\end{array}$ & $\begin{array}{l}7.7 \\
7.7 \\
7.7 \\
7.7 \\
7.7\end{array}$ & $\begin{array}{l}7.7 \\
7.6 \\
7.6 \\
7.7 \\
7.6\end{array}$ & $\begin{array}{l}7.7 \\
7.7 \\
7.7 \\
7.7 \\
7.7\end{array}$ \\
\hline $\begin{array}{l}26 \\
27 \\
28 \\
29 \\
30 \\
31\end{array}$ & $\begin{array}{l}7.6 \\
7.6 \\
7.5 \\
7.4 \\
7.4 \\
7.5\end{array}$ & $\begin{array}{l}7.3 \\
7.3 \\
7.4 \\
7.3 \\
7.3 \\
7.3\end{array}$ & $\begin{array}{l}7.5 \\
7.5 \\
7.4 \\
7.4 \\
7.4 \\
7.4\end{array}$ & $\begin{array}{c}7.6 \\
7.6 \\
7.6 \\
7.7 \\
7.7 \\
---\end{array}$ & $\begin{array}{l}7.5 \\
7.5 \\
7.6 \\
7.6 \\
7.6 \\
-\end{array}$ & $\begin{array}{l}7.5 \\
7.6 \\
7.6 \\
7.6 \\
7.6 \\
-.-\end{array}$ & $\begin{array}{l}7.9 \\
7.9 \\
7.9 \\
7.9 \\
7.9 \\
8.0\end{array}$ & $\begin{array}{l}7.9 \\
7.8 \\
7.9 \\
7.9 \\
7.9 \\
7.9\end{array}$ & $\begin{array}{l}7.9 \\
7.9 \\
7.9 \\
7.9 \\
7.9 \\
7.9\end{array}$ & $\begin{array}{l}7.7 \\
7.7 \\
7.6 \\
7.6 \\
7.7 \\
7.7\end{array}$ & $\begin{array}{l}7.6 \\
7.6 \\
7.5 \\
7.6 \\
7.6 \\
7.6\end{array}$ & $\begin{array}{l}7.6 \\
7.6 \\
7.6 \\
7.6 \\
7.7 \\
7.7\end{array}$ \\
\hline $\begin{array}{l}\text { MAX } \\
\text { MIN }\end{array}$ & $\begin{array}{l}8.0 \\
7.3\end{array}$ & $\begin{array}{l}7.5 \\
7.2\end{array}$ & $\begin{array}{l}7.7 \\
7.3\end{array}$ & $\begin{array}{l}7.8 \\
7.4\end{array}$ & $\begin{array}{l}7.7 \\
7.2\end{array}$ & $\begin{array}{l}7.8 \\
7.3\end{array}$ & $\begin{array}{l}8.0 \\
7.5\end{array}$ & $\begin{array}{l}7.9 \\
7.5\end{array}$ & $\begin{array}{l}7.9 \\
7.5\end{array}$ & $\begin{array}{l}8.0 \\
7.6\end{array}$ & $\begin{array}{l}7.9 \\
7.5\end{array}$ & $\begin{array}{l}8.0 \\
7.6\end{array}$ \\
\hline
\end{tabular}


ARKANSAS RTVER SUBBASTN

07178200 BIRD CREEK AT STATE HIGHWAY 266 NEAR CATOOSA, OKLABOMA--CONTINUED

PH, WATER, WHOLE, FIELD, STANDARD UNITS, WATER YEAR OCTOBER 1994 TO SEPTEMBER 1995 FEBRUARY

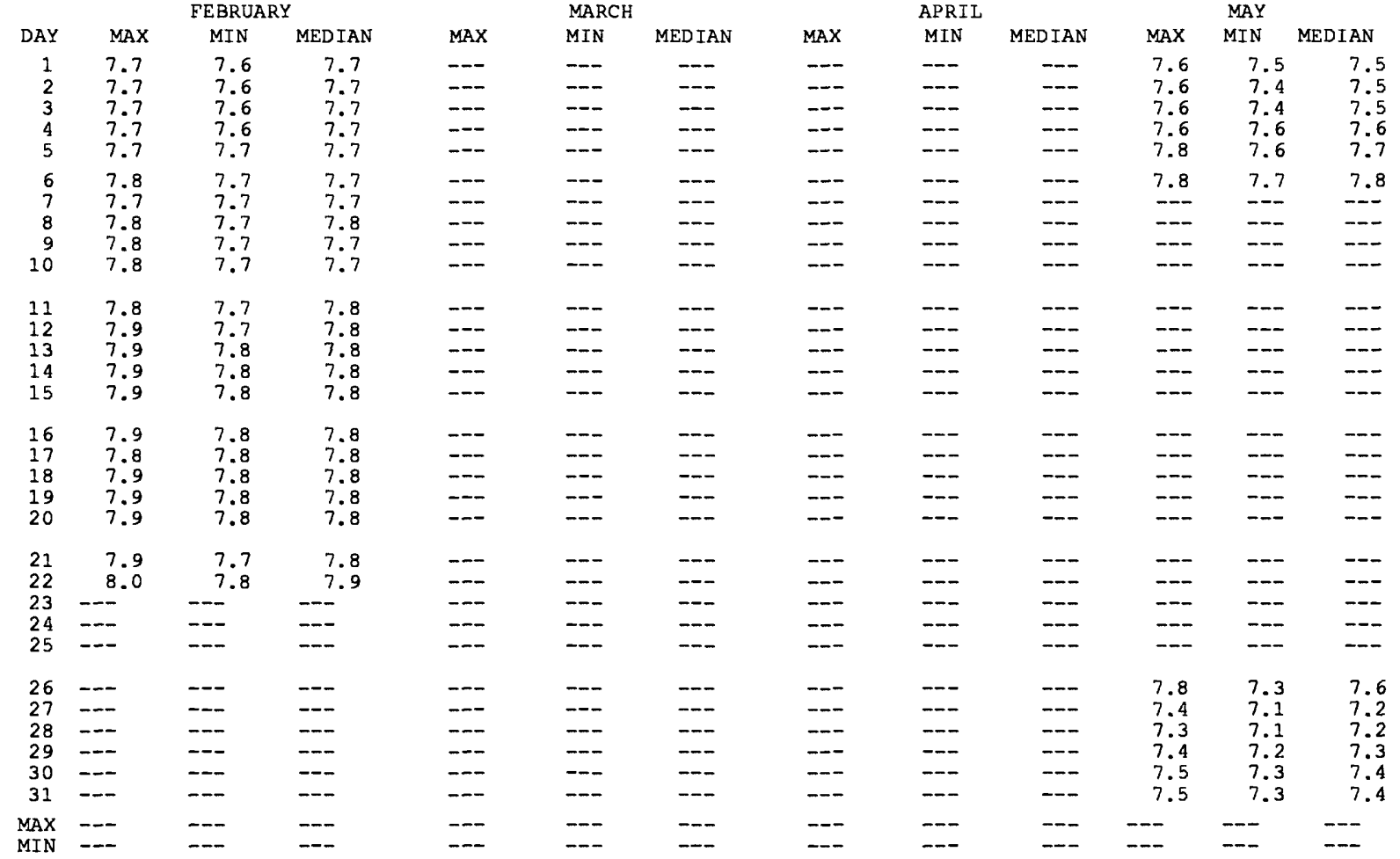

\begin{tabular}{|c|c|c|c|c|c|c|c|c|c|c|c|c|}
\hline \multirow[b]{2}{*}{ DAY } & \multicolumn{3}{|c|}{ JUNE } & \multicolumn{3}{|c|}{ JULY } & \multicolumn{3}{|c|}{ AUGUST } & \multicolumn{3}{|c|}{ SEPTEMBER } \\
\hline & $\operatorname{MAX}$ & MIN & MEDIAN & $\operatorname{MAX}$ & MIN & MEDIAN & $\operatorname{MAX}$ & MIN & MEDIAN & $\operatorname{MAX}$ & MIN & MEDIAN \\
\hline $\begin{array}{l}1 \\
2 \\
3 \\
4 \\
5\end{array}$ & $\begin{array}{l}7.5 \\
7.4 \\
7.5 \\
7.6 \\
7.6\end{array}$ & $\begin{array}{l}7.2 \\
7.2 \\
7.2 \\
7.2 \\
7.3\end{array}$ & $\begin{array}{l}7.3 \\
7.3 \\
7.5 \\
7.4 \\
7.5\end{array}$ & $\begin{array}{l}--- \\
--- \\
--- \\
---\end{array}$ & $\begin{array}{l}--- \\
--- \\
--- \\
---\end{array}$ & $\begin{array}{l}--- \\
--- \\
--- \\
---\end{array}$ & $\begin{array}{l}7.8 \\
7.6 \\
7.4 \\
7.4 \\
7.5\end{array}$ & $\begin{array}{l}7.4 \\
7.2 \\
7.2 \\
7.3 \\
7.4\end{array}$ & $\begin{array}{l}7.6 \\
7.3 \\
7.3 \\
7.3 \\
7.4\end{array}$ & $\begin{array}{l}7.6 \\
7.6 \\
7.6 \\
7.7 \\
7.8\end{array}$ & $\begin{array}{l}7.2 \\
7.4 \\
7.5 \\
7.4 \\
7.7\end{array}$ & $\begin{array}{l}7.4 \\
7.6 \\
7.6 \\
7.5 \\
7.7\end{array}$ \\
\hline $\begin{array}{r}6 \\
7 \\
8 \\
9 \\
10\end{array}$ & $\begin{array}{l}7.5 \\
7.5 \\
7.4 \\
7.8 \\
7.4\end{array}$ & $\begin{array}{l}7.2 \\
7.3 \\
7.2 \\
7.3 \\
7.1\end{array}$ & $\begin{array}{l}7.4 \\
7.4 \\
7.3 \\
7.5 \\
7.2\end{array}$ & $\begin{array}{l}--- \\
=- \\
-- \\
---\end{array}$ & $\begin{array}{l}--- \\
=- \\
--- \\
---\end{array}$ & $\begin{array}{l}--- \\
-- \\
-- \\
--\end{array}$ & $\begin{array}{l}7.4 \\
7.5 \\
7.4 \\
7.4 \\
7.3\end{array}$ & $\begin{array}{l}7.2 \\
7.1 \\
7.2 \\
7.2 \\
7.1\end{array}$ & $\begin{array}{l}7.3 \\
7.4 \\
7.3 \\
7.3 \\
7.2\end{array}$ & $\begin{array}{r}7.8 \\
7.9 \\
7.6 \\
7.6 \\
--\end{array}$ & $\begin{array}{r}7.7 \\
7.4 \\
7.4 \\
7.4 \\
---\end{array}$ & $\begin{array}{r}7.7 \\
7.5 \\
7.5 \\
7.6 \\
-\end{array}$ \\
\hline $\begin{array}{l}11 \\
12 \\
13 \\
14 \\
15\end{array}$ & $\begin{array}{r}7.4 \\
7.1 \\
-- \\
--\end{array}$ & \begin{tabular}{l}
7.1 \\
7.0 \\
-- \\
\hdashline-
\end{tabular} & $\begin{array}{r}7.2 \\
7.1 \\
-- \\
--\end{array}$ & $\begin{array}{r}-- \\
-7.3 \\
7.4 \\
7.7\end{array}$ & $\begin{array}{r}7.0 \\
7.2 \\
7.3\end{array}$ & $\begin{array}{r}-- \\
7.2 \\
7.3 \\
7.4\end{array}$ & $\begin{array}{l}7.3 \\
7.3 \\
7.4 \\
7.4 \\
7.5\end{array}$ & $\begin{array}{l}7.0 \\
7.1 \\
7.2 \\
7.1 \\
7.2\end{array}$ & $\begin{array}{l}7.2 \\
7.2 \\
7.3 \\
7.2 \\
7.3\end{array}$ & $\begin{array}{l}--- \\
-- \\
--- \\
---\end{array}$ & $\begin{array}{l}--- \\
--- \\
--- \\
--\end{array}$ & $\begin{array}{l}-- \\
--- \\
--- \\
--\end{array}$ \\
\hline $\begin{array}{l}16 \\
17 \\
18 \\
19 \\
20\end{array}$ & $\begin{array}{l}--- \\
--- \\
--\overline{7} \\
-3.3\end{array}$ & $\begin{array}{c}--- \\
\overline{-} \\
\overline{7.3} \\
-\end{array}$ & $\begin{array}{l}--- \\
=- \\
-\overline{7.3} \\
-\overline{7}\end{array}$ & $\begin{array}{l}7.6 \\
7.7 \\
7.7 \\
7.7 \\
7.5\end{array}$ & $\begin{array}{l}7.3 \\
7.5 \\
7.4 \\
7.2 \\
7.2\end{array}$ & $\begin{array}{l}7.6 \\
7.6 \\
7.6 \\
7.5 \\
7.3\end{array}$ & $\begin{array}{l}7.5 \\
7.6 \\
7.7 \\
7.5 \\
7.5\end{array}$ & $\begin{array}{l}7.3 \\
7.3 \\
7.2 \\
7.0 \\
7.0\end{array}$ & $\begin{array}{l}7.4 \\
7.5 \\
7.5 \\
7.2 \\
7.2\end{array}$ & $\begin{array}{l}--- \\
-- \\
-- \\
--\end{array}$ & $\begin{array}{l}--- \\
--- \\
-\cdots \\
---\end{array}$ & $\begin{array}{l}--- \\
--- \\
--- \\
--\end{array}$ \\
\hline $\begin{array}{l}21 \\
22 \\
23 \\
24 \\
25\end{array}$ & $\begin{array}{l}7.4 \\
7.3 \\
7.6 \\
7.4 \\
7.3\end{array}$ & $\begin{array}{l}7.3 \\
7.3 \\
7.2 \\
7.1 \\
7.2\end{array}$ & $\begin{array}{l}7.3 \\
7.3 \\
7.3 \\
7.2 \\
7.2\end{array}$ & $\begin{array}{l}7.5 \\
7.8 \\
7.4 \\
7.6 \\
7.5\end{array}$ & $\begin{array}{l}7.3 \\
7.4 \\
7.1 \\
7.4 \\
7.3\end{array}$ & $\begin{array}{l}7.4 \\
7.5 \\
7.4 \\
7.5 \\
7.3\end{array}$ & $\begin{array}{l}7.7 \\
7.7 \\
7.8 \\
7.9 \\
7.9\end{array}$ & $\begin{array}{l}7.3 \\
7.6 \\
7.5 \\
7.6 \\
7.6\end{array}$ & $\begin{array}{l}7.6 \\
7.7 \\
7.6 \\
7.8 \\
7.8\end{array}$ & $\begin{array}{l}--- \\
--- \\
--- \\
---\end{array}$ & $\begin{array}{l}--- \\
--- \\
-- \\
--\end{array}$ & $\begin{array}{l}-- \\
--- \\
--- \\
--\end{array}$ \\
\hline $\begin{array}{l}26 \\
27 \\
28 \\
29 \\
30 \\
31\end{array}$ & $\begin{array}{c}7.4 \\
7.4 \\
7.4 \\
7.6 \\
---\end{array}$ & $\begin{array}{l}7.2 \\
7.3 \\
7.4 \\
7.2 \\
-- \\
--\end{array}$ & $\begin{array}{c}7.3 \\
7.3 \\
7.4 \\
7.4 \\
-- \\
--\end{array}$ & $\begin{array}{l}7.5 \\
7.5 \\
7.6 \\
7.6 \\
7.7 \\
7.8\end{array}$ & $\begin{array}{l}7.3 \\
7.4 \\
7.3 \\
7.3 \\
7.2 \\
7.6\end{array}$ & $\begin{array}{l}7.4 \\
7.5 \\
7.5 \\
7.5 \\
7.6 \\
7.7\end{array}$ & $\begin{array}{l}7.9 \\
7.8 \\
7.9 \\
7.9 \\
7.9 \\
7.5\end{array}$ & $\begin{array}{l}7.7 \\
7.5 \\
7.5 \\
7.6 \\
7.3 \\
7.2\end{array}$ & $\begin{array}{l}7.8 \\
7.8 \\
7.8 \\
7.8 \\
7.5 \\
7.3\end{array}$ & $\begin{array}{l}--- \\
--- \\
--- \\
-- \\
--\end{array}$ & $\begin{array}{l}--- \\
--- \\
--- \\
--- \\
---\end{array}$ & $\begin{array}{l}--- \\
--- \\
--- \\
--- \\
--\end{array}$ \\
\hline $\begin{array}{l}\text { AX } \\
\text { IN }\end{array}$ & -- & $=-$ & $=-$ & $=-$ & $=-$ & --- & $\begin{array}{l}7.9 \\
7.3\end{array}$ & $\begin{array}{l}7.7 \\
7.0\end{array}$ & $\begin{array}{l}7.8 \\
7.2\end{array}$ & $=-$ & $\cdots$ & $=-$ \\
\hline
\end{tabular}


ARKANSAS RIVER SUBBASIN

07178200 BIRD CREEK AT STATE HIGHMAY 266 NEAR CATOOSA, OKLABOMA--CONTINOED

OXYGEN DISSOLVED (MG/L), WATER YEAR OCTOBER 1994 TO SEPTEMBER 1995

\begin{tabular}{|c|c|c|c|c|c|c|c|c|c|c|c|c|}
\hline \multirow[b]{2}{*}{ DAY } & \multicolumn{3}{|c|}{ OCTOBER } & \multicolumn{3}{|c|}{$\begin{array}{l}M(M / L), \\
\text { NOVEMBER }\end{array}$} & \multicolumn{3}{|c|}{ DECEMBER } & \multicolumn{3}{|c|}{ JANUARY } \\
\hline & MAX & MIN & MEAN & $\operatorname{MAX}$ & MIN & MEAN & MAX & MIN & MEAN & MAX & MIN & MEAN \\
\hline $\begin{array}{l}1 \\
2 \\
3 \\
4 \\
5\end{array}$ & $\begin{array}{r}--- \\
--- \\
-- \\
10.0 \\
8.7\end{array}$ & $\begin{array}{l}--- \\
--- \\
-- \\
7.8 \\
6.2\end{array}$ & $\begin{array}{r}--- \\
--- \\
--- \\
9.0 \\
6.7\end{array}$ & $\begin{array}{r}8.1 \\
8.3 \\
10.0 \\
8.0 \\
8.8\end{array}$ & $\begin{array}{l}7.9 \\
7.9 \\
7.6 \\
6.6 \\
5.0\end{array}$ & $\begin{array}{l}8.0 \\
8.1 \\
8.1 \\
7.2 \\
7.2\end{array}$ & $\begin{array}{r}14.0 \\
10.7 \\
9.6 \\
9.2\end{array}$ & $\begin{array}{r}10.4 \\
9.4 \\
9.1 \\
8.9\end{array}$ & $\begin{array}{r}12.0 \\
9.9 \\
9.4 \\
9.1\end{array}$ & $\begin{array}{l}11.7 \\
11.9 \\
12.1 \\
12.7 \\
12.9\end{array}$ & $\begin{array}{l}10.4 \\
11.0 \\
11.2 \\
11.7 \\
12.1\end{array}$ & $\begin{array}{l}11.0 \\
11.5 \\
11.6 \\
12.1 \\
12.6\end{array}$ \\
\hline $\begin{array}{r}6 \\
7 \\
8 \\
9 \\
10\end{array}$ & $\begin{array}{l}6.7 \\
6.9 \\
6.8 \\
6.9 \\
8.1\end{array}$ & $\begin{array}{l}5.8 \\
5.6 \\
6.2 \\
6.4 \\
6.4\end{array}$ & $\begin{array}{l}6.3 \\
6.0 \\
6.5 \\
6.7 \\
6.9\end{array}$ & $\begin{array}{l}--- \\
--- \\
--- \\
--- \\
---\end{array}$ & $\begin{array}{l}--- \\
--- \\
--- \\
--- \\
---\end{array}$ & $\begin{array}{l}--- \\
--- \\
--- \\
--- \\
---\end{array}$ & $\begin{array}{r}9.2 \\
9.7 \\
10.2 \\
10.8 \\
11.1\end{array}$ & $\begin{array}{r}9.1 \\
9.0 \\
9.6 \\
10.2 \\
10.8\end{array}$ & $\begin{array}{r}9.1 \\
9.3 \\
9.8 \\
10.5 \\
11.0\end{array}$ & $\begin{array}{l}12.9 \\
13.0 \\
12.9 \\
12.7 \\
12.5\end{array}$ & $\begin{array}{l}11.8 \\
11.8 \\
12.1 \\
11.9 \\
11.8\end{array}$ & $\begin{array}{l}12.1 \\
12.3 \\
12.5 \\
12.4 \\
12.1\end{array}$ \\
\hline $\begin{array}{l}11 \\
12 \\
13 \\
14 \\
15\end{array}$ & $\begin{array}{c}7.9 \\
7.1 \\
--- \\
--- \\
---\end{array}$ & $\begin{array}{c}6.8 \\
6.7 \\
--- \\
---\end{array}$ & $\begin{array}{c}7.1 \\
7.0 \\
-- \\
--\end{array}$ & $\begin{array}{l}--- \\
--- \\
--- \\
--- \\
---\end{array}$ & $\begin{array}{l}--- \\
--- \\
--- \\
--- \\
---\end{array}$ & $\begin{array}{l}--- \\
--- \\
--- \\
--- \\
---\end{array}$ & $\begin{array}{l}11.1 \\
11.2 \\
11.0 \\
10.9 \\
10.8\end{array}$ & $\begin{array}{l}11.0 \\
11.0 \\
10.8 \\
10.6 \\
10.5\end{array}$ & $\begin{array}{l}11.0 \\
11.1 \\
10.9 \\
10.7 \\
10.7\end{array}$ & $\begin{array}{l}12.2 \\
11.2 \\
11.1 \\
11.0 \\
11.0\end{array}$ & $\begin{array}{r}11.0 \\
10.3 \\
9.9 \\
9.8 \\
10.1\end{array}$ & $\begin{array}{l}11.4 \\
10.7 \\
10.4 \\
10.3 \\
10.6\end{array}$ \\
\hline $\begin{array}{l}16 \\
17 \\
18 \\
19 \\
20\end{array}$ & $\begin{array}{l}--- \\
--- \\
--- \\
--\overline{7.7}\end{array}$ & $\begin{array}{l}--- \\
--- \\
--- \\
--\overline{6.9}\end{array}$ & $\begin{array}{l}--- \\
--- \\
--- \\
--\overline{7.3}\end{array}$ & $\begin{array}{r}--- \\
--- \\
9.2 \\
9.0 \\
---\end{array}$ & $\begin{array}{r}--- \\
-- \\
9.0 \\
8.4 \\
---\end{array}$ & $\begin{array}{r}-- \\
-- \\
9.1 \\
---\end{array}$ & $\begin{array}{l}11.0 \\
10.9 \\
11.0 \\
11.0 \\
11.0\end{array}$ & $\begin{array}{l}10.5 \\
10.6 \\
10.6 \\
10.7 \\
10.6\end{array}$ & $\begin{array}{l}10.8 \\
10.8 \\
10.8 \\
10.8 \\
10.9\end{array}$ & $\begin{array}{l}11.0 \\
10.8 \\
10.0 \\
10.7 \\
10.6\end{array}$ & $\begin{array}{r}10.1 \\
9.6 \\
9.3 \\
9.5 \\
10.1\end{array}$ & $\begin{array}{r}10.6 \\
10.2 \\
9.6 \\
10.2 \\
10.3\end{array}$ \\
\hline $\begin{array}{l}21 \\
22 \\
23 \\
24 \\
25\end{array}$ & $\begin{array}{l}7.1 \\
7.2 \\
7.2 \\
8.3 \\
9.0\end{array}$ & $\begin{array}{l}6.6 \\
6.7 \\
6.6 \\
6.7 \\
6.8\end{array}$ & $\begin{array}{l}6.8 \\
6.9 \\
6.9 \\
7.3 \\
7.4\end{array}$ & $\begin{array}{l}--- \\
--- \\
--- \\
--- \\
---\end{array}$ & $\begin{array}{l}--- \\
--- \\
--- \\
--- \\
---\end{array}$ & $\begin{array}{l}--- \\
--- \\
--- \\
--- \\
---\end{array}$ & $\begin{array}{l}11.0 \\
10.8 \\
10.8 \\
11.0 \\
11.2\end{array}$ & $\begin{array}{l}10.5 \\
10.3 \\
10.4 \\
10.5 \\
10.7\end{array}$ & $\begin{array}{l}10.7 \\
10.5 \\
10.6 \\
10.8 \\
11.0\end{array}$ & $\begin{array}{l}10.9 \\
11.1 \\
12.0 \\
12.0 \\
11.8\end{array}$ & $\begin{array}{r}9.9 \\
10.4 \\
11.1 \\
11.0 \\
10.8\end{array}$ & $\begin{array}{l}10.4 \\
10.8 \\
11.5 \\
11.5 \\
11.4\end{array}$ \\
\hline $\begin{array}{l}26 \\
27 \\
28 \\
29 \\
30 \\
31\end{array}$ & $\begin{array}{r}-- \\
8.8 \\
8.0 \\
8.0 \\
7.9\end{array}$ & $\begin{array}{r}-- \\
-8.0 \\
7.7 \\
7.6 \\
7.5\end{array}$ & $\begin{array}{r}-- \\
8.5 \\
7.9 \\
7.8 \\
7.7\end{array}$ & $\begin{array}{l}--- \\
--- \\
--- \\
--- \\
--- \\
---\end{array}$ & $\begin{array}{l}--- \\
--- \\
--- \\
--- \\
--- \\
---\end{array}$ & $\begin{array}{l}--- \\
--- \\
--- \\
--- \\
--- \\
---\end{array}$ & $\begin{array}{l}11.4 \\
11.4 \\
11.3 \\
11.1 \\
11.3 \\
11.3\end{array}$ & $\begin{array}{l}11.0 \\
11.0 \\
10.9 \\
10.6 \\
10.7 \\
10.5\end{array}$ & $\begin{array}{l}11.2 \\
11.2 \\
11.1 \\
10.9 \\
11.0 \\
10.8\end{array}$ & $\begin{array}{l}11.8 \\
11.1 \\
--- \\
--- \\
---\end{array}$ & $\begin{array}{l}10.8 \\
10.1 \\
--- \\
--- \\
---\end{array}$ & $\begin{array}{l}11.1 \\
10.3 \\
-\cdots \\
-- \\
--\end{array}$ \\
\hline \multirow[t]{2}{*}{ MONTH } & --- & --- & --- & --- & --- & -- & --- & -- & $-\cdots$ & --- & --- & $-\infty$ \\
\hline & \multicolumn{3}{|c|}{ FEBRUARY } & \multicolumn{3}{|c|}{ MARCH } & \multicolumn{3}{|c|}{ APRIL } & \multicolumn{3}{|c|}{ MAY } \\
\hline DAY & $\operatorname{MAX}$ & $\mathrm{MIN}$ & MEAN & $\operatorname{MAX}$ & MIN & MEAN & $\operatorname{MAX}$ & MIN & MEAN & MAX & MIN & MEAN \\
\hline $\begin{array}{l}1 \\
2 \\
3 \\
4 \\
5\end{array}$ & $\begin{array}{l}--- \\
10.9 \\
10.9 \\
11.1 \\
11.1\end{array}$ & $\begin{array}{r}10.5 \\
9.9 \\
9.9 \\
10.2\end{array}$ & $\begin{array}{l}-- \\
10.7 \\
10.3 \\
10.5 \\
10.7\end{array}$ & $\begin{array}{l}12.0 \\
-- \\
-- \\
--\end{array}$ & $\begin{array}{l}10.7 \\
--- \\
--- \\
--- \\
---\end{array}$ & $\begin{array}{l}11.3 \\
-- \\
-- \\
--\end{array}$ & $\begin{array}{r}9.6 \\
9.5 \\
9.4 \\
9.7 \\
--\end{array}$ & $\begin{array}{r}8.4 \\
8.1 \\
7.7 \\
8.1 \\
--\end{array}$ & $\begin{array}{r}9.1 \\
8.9 \\
8.4 \\
8.9 \\
-\end{array}$ & $\begin{array}{l}8.5 \\
9.1 \\
9.4 \\
9.4 \\
9.7\end{array}$ & $\begin{array}{l}7.6 \\
8.5 \\
9.0 \\
9.2 \\
9.4\end{array}$ & $\begin{array}{l}8.0 \\
8.8 \\
9.3 \\
9.3 \\
9.6\end{array}$ \\
\hline $\begin{array}{r}6 \\
7 \\
8 \\
9 \\
10\end{array}$ & $\begin{array}{l}11.1 \\
11.2 \\
11.8 \\
11.8 \\
11.7\end{array}$ & $\begin{array}{l}10.4 \\
10.5 \\
10.9 \\
10.9 \\
10.7\end{array}$ & $\begin{array}{l}10.8 \\
10.9 \\
11.3 \\
11.4 \\
11.2\end{array}$ & $\begin{array}{l}--- \\
--- \\
--- \\
--- \\
13.5\end{array}$ & $\begin{array}{l}--- \\
--- \\
--- \\
--- \\
11.9\end{array}$ & $\begin{array}{l}--- \\
--- \\
--- \\
--- \\
12.7\end{array}$ & $\begin{array}{r}9.6 \\
9.8 \\
10.0 \\
8.4\end{array}$ & $\begin{array}{r}8.7 \\
9.2 \\
9.3 \\
6.9\end{array}$ & $\begin{array}{r}9.0 \\
9.5 \\
9.6 \\
7.5\end{array}$ & $\begin{array}{l}9.6 \\
9.2 \\
7.9 \\
7.2 \\
6.1\end{array}$ & $\begin{array}{l}8.6 \\
7.8 \\
7.2 \\
6.1 \\
5.3\end{array}$ & $\begin{array}{l}9.4 \\
8.4 \\
7.6 \\
6.8 \\
5.5\end{array}$ \\
\hline $\begin{array}{l}11 \\
12 \\
13 \\
14 \\
15\end{array}$ & $\begin{array}{l}-- \\
--- \\
--\end{array}$ & $\begin{array}{l}--- \\
--- \\
--- \\
--- \\
---\end{array}$ & $\begin{array}{l}--- \\
--- \\
---\end{array}$ & $\begin{array}{r}11.9 \\
11.0 \\
11.8 \\
11.8 \\
9.4\end{array}$ & $\begin{array}{r}11.0 \\
10.5 \\
9.0 \\
8.4 \\
8.5\end{array}$ & $\begin{array}{r}11.4 \\
10.7 \\
10.1 \\
9.0 \\
8.8\end{array}$ & $\begin{array}{l}7.4 \\
6.3 \\
5.9 \\
5.8 \\
5.9\end{array}$ & $\begin{array}{l}6.3 \\
5.9 \\
5.7 \\
5.7 \\
5.7\end{array}$ & $\begin{array}{l}6.7 \\
6.0 \\
5.8 \\
5.7 \\
5.8\end{array}$ & $\begin{array}{l}9.0 \\
9.1 \\
9.0 \\
9.3 \\
9.5\end{array}$ & $\begin{array}{l}5.6 \\
8.9 \\
8.9 \\
8.8 \\
9.2\end{array}$ & $\begin{array}{l}8.0 \\
9.0 \\
9.0 \\
9.1 \\
9.3\end{array}$ \\
\hline $\begin{array}{l}16 \\
17 \\
18 \\
19 \\
20\end{array}$ & $\begin{array}{l}--- \\
--- \\
--- \\
---\end{array}$ & $\begin{array}{l}--- \\
--- \\
--- \\
--- \\
---\end{array}$ & $\begin{array}{l}--- \\
--- \\
--- \\
---\end{array}$ & $\begin{array}{l}11.9 \\
12.1 \\
11.9 \\
11.7 \\
11.5\end{array}$ & $\begin{array}{r}9.3 \\
11.5 \\
11.2 \\
11.2 \\
10.6\end{array}$ & $\begin{array}{l}11.0 \\
11.8 \\
11.6 \\
11.4 \\
11.1\end{array}$ & $\begin{array}{l}6.4 \\
7.0 \\
7.2 \\
8.1 \\
8.8\end{array}$ & $\begin{array}{l}5.8 \\
5.1 \\
5.8 \\
6.6 \\
8.2\end{array}$ & $\begin{array}{l}6.0 \\
5.8 \\
6.8 \\
7.2 \\
8.6\end{array}$ & $\begin{array}{l}9.3 \\
9.1 \\
9.2 \\
9.1 \\
9.5\end{array}$ & $\begin{array}{l}9.1 \\
8.0 \\
7.6 \\
8.1 \\
9.1\end{array}$ & $\begin{array}{l}9.2 \\
8.8 \\
8.4 \\
8.6 \\
9.4\end{array}$ \\
\hline $\begin{array}{l}21 \\
22 \\
23 \\
24 \\
25\end{array}$ & $\begin{array}{l}-- \\
14.3 \\
11.6 \\
12.7 \\
13.7\end{array}$ & $\begin{array}{l}-10.7 \\
10.3 \\
10.0 \\
10.5\end{array}$ & $\begin{array}{l}-- \\
12.5 \\
10.9 \\
11.0 \\
11.6\end{array}$ & $\begin{array}{r}10.9 \\
10.4 \\
9.6 \\
9.0 \\
8.7\end{array}$ & $\begin{array}{r}10.1 \\
9.6 \\
8.9 \\
8.6 \\
8.2\end{array}$ & $\begin{array}{r}10.5 \\
10.0 \\
9.2 \\
8.8 \\
8.5\end{array}$ & $\begin{array}{c}9.1 \\
8.8 \\
-- \\
-9.6\end{array}$ & $\begin{array}{c}8.7 \\
8.6 \\
--- \\
-- \\
9.1\end{array}$ & $\begin{array}{c}8.8 \\
8.7 \\
9 .\end{array}$ & $\begin{array}{l}9.6 \\
9.9 \\
9.9 \\
9.6 \\
9.2\end{array}$ & $\begin{array}{l}9.4 \\
9.4 \\
9.6 \\
8.0 \\
8.3\end{array}$ & $\begin{array}{l}9.5 \\
9.6 \\
9.7 \\
9.0 \\
8.6\end{array}$ \\
\hline $\begin{array}{l}26 \\
27 \\
28 \\
29 \\
30 \\
31\end{array}$ & $\begin{array}{l}10.8 \\
10.9 \\
10.7 \\
--- \\
---\end{array}$ & $\begin{array}{r}9.7 \\
8.0 \\
8.7 \\
-- \\
--\end{array}$ & $\begin{array}{c}10.3 \\
9.8 \\
9.8 \\
--- \\
---\end{array}$ & $\begin{array}{l}9.0 \\
9.8 \\
9.6 \\
9.7 \\
9.8 \\
9.8\end{array}$ & $\begin{array}{l}7.9 \\
8.1 \\
8.3 \\
8.2 \\
8.4 \\
8.6\end{array}$ & $\begin{array}{l}8.4 \\
9.0 \\
9.1 \\
9.0 \\
9.2 \\
9.3\end{array}$ & $\begin{array}{r}9.8 \\
10.1 \\
10.7 \\
10.2 \\
8.8 \\
--\end{array}$ & $\begin{array}{r}9.5 \\
9.6 \\
10.1 \\
8.5 \\
7.6 \\
--\end{array}$ & $\begin{array}{r}9.7 \\
9.8 \\
10.5 \\
9.2 \\
7.9 \\
--\end{array}$ & $\begin{array}{l}8.8 \\
7.7 \\
7.1 \\
8.3 \\
9.0 \\
8.9\end{array}$ & $\begin{array}{l}7.3 \\
7.1 \\
6.6 \\
6.9 \\
8.3 \\
8.0\end{array}$ & $\begin{array}{l}8.3 \\
7.3 \\
6.7 \\
7.7 \\
8.8 \\
8.5\end{array}$ \\
\hline MONTH & --- & $-\infty$ & --- & --- & --- & -- & $\cdots$ & --- & -- & 9.9 & 5.3 & 8.6 \\
\hline
\end{tabular}


ARKANSAS RIVGR SUBBASIN

07178200 BIRD CRERK AT STATE BIGEWAY 266 NHAR CATOOSA, OKLABOMA--CONTINUED

OXYGEN DISSOLVED (MG/L), WATER YEAR OCTOBER 1994 TO SEPTEMBER 1995

\begin{tabular}{|c|c|c|c|c|c|c|c|c|c|c|c|c|}
\hline \multirow[b]{2}{*}{ DAY } & \multicolumn{3}{|c|}{ JUNE } & \multicolumn{3}{|c|}{ JULY } & \multicolumn{3}{|c|}{ AUGUST } & \multicolumn{3}{|c|}{ SEPTEMBER } \\
\hline & MAX & MIN & MEAN & $\operatorname{MAX}$ & MIN & MEAN & MAX & MIN & MEAN & $\operatorname{MAX}$ & MIN & MEAN \\
\hline $\begin{array}{l}1 \\
2 \\
3 \\
4 \\
5\end{array}$ & $\begin{array}{l}8.3 \\
7.7 \\
8.3 \\
7.5 \\
7.1\end{array}$ & $\begin{array}{l}7.5 \\
6.5 \\
6.7 \\
6.7 \\
6.7\end{array}$ & $\begin{array}{l}7.9 \\
7.0 \\
7.8 \\
7.0 \\
6.9\end{array}$ & $\begin{array}{l}8.3 \\
8.5 \\
8.5 \\
6.8 \\
6.0\end{array}$ & $\begin{array}{l}6.3 \\
8.3 \\
6.7 \\
6.0 \\
4.6\end{array}$ & $\begin{array}{l}7.7 \\
8.4 \\
8.1 \\
6.3 \\
5.4\end{array}$ & $\begin{array}{l}6.9 \\
6.5 \\
6.5 \\
6.7 \\
6.9\end{array}$ & $\begin{array}{l}6.2 \\
5.2 \\
6.0 \\
6.4 \\
6.7\end{array}$ & $\begin{array}{l}6.5 \\
5.8 \\
6.2 \\
6.6 \\
6.8\end{array}$ & $\begin{array}{l}6.3 \\
6.4 \\
6.2 \\
6.0 \\
6.2\end{array}$ & $\begin{array}{l}5.5 \\
5.5 \\
5.4 \\
5.4 \\
5.6\end{array}$ & $\begin{array}{l}6.0 \\
5.9 \\
5.7 \\
5.7 \\
5.9\end{array}$ \\
\hline $\begin{array}{r}6 \\
7 \\
8 \\
9 \\
10\end{array}$ & $\begin{array}{l}7.1 \\
7.0 \\
6.1 \\
7.5 \\
7.2\end{array}$ & $\begin{array}{l}6.4 \\
6.1 \\
4.7 \\
5.0 \\
6.5\end{array}$ & $\begin{array}{l}6.8 \\
6.5 \\
5.3 \\
6.6 \\
6.8\end{array}$ & $\begin{array}{l}7.7 \\
8.3 \\
8.4 \\
8.5 \\
8.7\end{array}$ & $\begin{array}{l}4.6 \\
7.7 \\
8.1 \\
8.1 \\
8.2\end{array}$ & $\begin{array}{l}6.4 \\
8.1 \\
8.2 \\
8.3 \\
8.4\end{array}$ & $\begin{array}{l}7.1 \\
7.0 \\
7.1 \\
7.2 \\
7.1\end{array}$ & $\begin{array}{l}6.7 \\
6.7 \\
6.9 \\
6.9 \\
6.9\end{array}$ & $\begin{array}{l}7.0 \\
6.9 \\
7.0 \\
7.0 \\
7.0\end{array}$ & $\begin{array}{l}6.2 \\
6.2 \\
6.2 \\
6.4 \\
6.8\end{array}$ & $\begin{array}{l}5.5 \\
4.0 \\
3.3 \\
5.6 \\
6.4\end{array}$ & $\begin{array}{l}5.9 \\
5.5 \\
5.3 \\
6.0 \\
6.6\end{array}$ \\
\hline $\begin{array}{l}11 \\
12 \\
13 \\
14 \\
15\end{array}$ & $\begin{array}{l}6.8 \\
6.3 \\
7.3 \\
7.5 \\
7.4\end{array}$ & $\begin{array}{l}6.3 \\
4.8 \\
5.1 \\
7.3 \\
7.3\end{array}$ & $\begin{array}{l}6.6 \\
5.2 \\
6.4 \\
7.4 \\
7.4\end{array}$ & $\begin{array}{l}8.6 \\
8.5 \\
8.5 \\
8.6 \\
8.4\end{array}$ & $\begin{array}{l}8.3 \\
8.3 \\
8.3 \\
8.4 \\
7.8\end{array}$ & $\begin{array}{l}8.4 \\
8.3 \\
8.4 \\
8.5 \\
8.1\end{array}$ & $\begin{array}{l}7.0 \\
6.8 \\
6.8 \\
6.6 \\
6.6\end{array}$ & $\begin{array}{l}6.6 \\
6.3 \\
6.0 \\
5.9 \\
5.9\end{array}$ & $\begin{array}{l}6.7 \\
6.5 \\
6.4 \\
6.3 \\
6.2\end{array}$ & $\begin{array}{l}6.7 \\
6.8 \\
6.3 \\
6.4 \\
6.5\end{array}$ & $\begin{array}{l}6.5 \\
6.0 \\
6.0 \\
6.1 \\
6.1\end{array}$ & $\begin{array}{l}6.6 \\
6.5 \\
6.2 \\
6.3 \\
6.3\end{array}$ \\
\hline $\begin{array}{l}16 \\
17 \\
18 \\
19 \\
20\end{array}$ & $\begin{array}{l}8.4 \\
8.5 \\
8.5 \\
8.4 \\
8.4\end{array}$ & $\begin{array}{l}7.0 \\
8.3 \\
8.3 \\
8.2 \\
8.2\end{array}$ & $\begin{array}{l}7.5 \\
8.4 \\
8.4 \\
8.3 \\
8.3\end{array}$ & $\begin{array}{l}8.2 \\
8.2 \\
8.2 \\
8.2 \\
8.0\end{array}$ & $\begin{array}{l}7.9 \\
7.8 \\
7.9 \\
7.9 \\
7.3\end{array}$ & $\begin{array}{l}8.1 \\
8.0 \\
8.1 \\
8.1 \\
7.7\end{array}$ & $\begin{array}{l}6.5 \\
6.5 \\
6.4 \\
6.5 \\
6.5\end{array}$ & $\begin{array}{l}5.8 \\
5.9 \\
5.8 \\
5.7 \\
5.7\end{array}$ & $\begin{array}{l}6.2 \\
6.2 \\
6.1 \\
6.1 \\
6.1\end{array}$ & $\begin{array}{l}6.5 \\
6.7 \\
6.5 \\
6.6 \\
7.0\end{array}$ & $\begin{array}{l}5.9 \\
5.3 \\
5.9 \\
6.5 \\
6.6\end{array}$ & $\begin{array}{l}6.3 \\
6.0 \\
6.3 \\
6.6 \\
6.8\end{array}$ \\
\hline $\begin{array}{l}21 \\
22 \\
23 \\
24 \\
25\end{array}$ & $\begin{array}{l}8.2 \\
8.2 \\
8.1 \\
7.7 \\
7.5\end{array}$ & $\begin{array}{l}8.0 \\
8.0 \\
6.9 \\
5.1 \\
7.0\end{array}$ & $\begin{array}{l}8.1 \\
8.1 \\
7.8 \\
6.8 \\
7.3\end{array}$ & $\begin{array}{l}8.1 \\
8.0 \\
7.9 \\
7.9 \\
8.0\end{array}$ & $\begin{array}{l}7.6 \\
6.5 \\
7.7 \\
7.5 \\
7.6\end{array}$ & $\begin{array}{l}7.9 \\
7.5 \\
7.8 \\
7.7 \\
7.8\end{array}$ & $\begin{array}{l}6.4 \\
6.4 \\
6.4 \\
6.8 \\
6.9\end{array}$ & $\begin{array}{l}5.6 \\
5.6 \\
5.5 \\
5.7 \\
5.9\end{array}$ & $\begin{array}{l}6.0 \\
6.0 \\
6.0 \\
6.2 \\
6.4\end{array}$ & $\begin{array}{l}7.5 \\
8.1 \\
8.4 \\
8.6 \\
8.5\end{array}$ & $\begin{array}{l}7.0 \\
7.5 \\
8.0 \\
8.1 \\
8.3\end{array}$ & $\begin{array}{l}7.2 \\
7.8 \\
8.2 \\
8.4 \\
8.4\end{array}$ \\
\hline $\begin{array}{l}26 \\
27 \\
28 \\
29 \\
30 \\
31\end{array}$ & $\begin{array}{r}7.8 \\
7.9 \\
7.9 \\
8.2 \\
6.5 \\
---\end{array}$ & $\begin{array}{r}7.4 \\
7.7 \\
7.7 \\
6.3 \\
6.1 \\
---\end{array}$ & $\begin{array}{r}7.6 \\
7.8 \\
7.8 \\
7.6 \\
6.3 \\
---\end{array}$ & $\begin{array}{l}7.8 \\
7.6 \\
7.3 \\
6.9 \\
6.8 \\
6.8\end{array}$ & $\begin{array}{l}6.9 \\
7.1 \\
6.9 \\
6.6 \\
6.3 \\
6.2\end{array}$ & $\begin{array}{l}7.2 \\
7.4 \\
7.1 \\
6.8 \\
6.5 \\
6.5\end{array}$ & $\begin{array}{l}6.9 \\
6.8 \\
6.8 \\
6.7 \\
6.6 \\
6.4\end{array}$ & $\begin{array}{l}6.0 \\
5.8 \\
5.9 \\
5.7 \\
5.7 \\
5.6\end{array}$ & $\begin{array}{l}6.6 \\
6.3 \\
6.4 \\
6.3 \\
6.1 \\
6.1\end{array}$ & $\begin{array}{r}9.0 \\
8.9 \\
8.9 \\
7.5 \\
7.1 \\
---\end{array}$ & $\begin{array}{r}8.4 \\
8.2 \\
7.4 \\
7.0 \\
6.8 \\
---\end{array}$ & $\begin{array}{r}8.6 \\
8.6 \\
8.0 \\
7.1 \\
6.9 \\
---\end{array}$ \\
\hline ITH & 8.5 & 4.7 & 7.3 & 8.7 & 4.6 & 7.7 & 7.2 & 5.2 & 6.4 & 9.0 & 3.3 & 6.7 \\
\hline
\end{tabular}




\section{SPAVINAW CREEK NEAR SYCAMORE, OKIAAOMA}

IOCArION.--Lat $36^{\circ} 20^{\prime} 07^{\prime \prime}$, long $94^{\circ} 38^{\prime} 27^{\prime \prime}$, in NE1/4NW1/4, sec.4, T.21 N., R.25 E., Delaware County, on right bank 1.8 mi upstream from Cherokeé creek, $4.8 \mathrm{ml}$ northeast of Row, $6.5 \mathrm{mi}$ southeast of Sycamore, and at mile 35.0 .

DRATDAE ARBA. $--133 \mathrm{mi}^{2}$.

AVERACE DISCHAREE. --34 years, $116 \mathrm{ft}^{3} / \mathrm{s}$.

EXIREMES.-October 1961 to current year: Maximum discharge 39,800 ft ${ }^{3} / \mathrm{s}$ July 27, $1975 ; \mathrm{minimum} 1.2 \mathrm{ft} / \mathrm{s}$ Aug. 9, 1964 . REMRRKS. --Records good.

Monthly and yearly discharge

\begin{tabular}{|c|c|c|c|c|c|}
\hline Month & $\begin{array}{l}\text { Total } \\
\left(\mathrm{ft}^{3} / \mathrm{s}\right)\end{array}$ & $\begin{array}{l}\text { Maximum } \\
\mathrm{dail}^{2} \mathrm{y} \\
\left(\mathrm{ft}^{3} / \mathrm{s}\right)\end{array}$ & $\begin{array}{l}\text { Minimum } \\
\text { daily } \\
\left(\mathrm{ft}^{3} / \mathrm{s}\right)\end{array}$ & $\begin{array}{c}\text { Mean } \\
\left(\mathrm{ft}^{3} / \mathrm{s}\right)\end{array}$ & $\begin{array}{c}\text { Runoff } \\
\text { (acre-feet) }\end{array}$ \\
\hline October & 739 & 28 & 20 & 23.8 & 1,470 \\
\hline November & 5,496 & 824 & 23 & 183 & 10,900 \\
\hline December & 2,548 & 207 & 51 & 82.2 & 5,050 \\
\hline January & 6,083 & 1,250 & 41 & 196 & 12,070 \\
\hline February & 2,635 & 133 & 74 & 94.1 & 5,230 \\
\hline March & 4,325 & 490 & 76 & 140 & 8,580 \\
\hline April & 6,910 & 844 & 68 & 230 & 13,710 \\
\hline May & 12,365 & 2,76 & 140 & 399 & 24,530 \\
\hline June & 14,592 & 4,520 & 116 & 486 & 28,940 \\
\hline July & 2,833 & 131 & 69 & 91.4 & 5,620 \\
\hline August & 1,541 & 68 & 31 & 49.7 & 3,060 \\
\hline September & 1,056 & 61 & 26 & 35.2 & 2,090 \\
\hline Water year 1995 & 61,123 & 4,520 & 20 & 167 & 121,200 \\
\hline
\end{tabular}




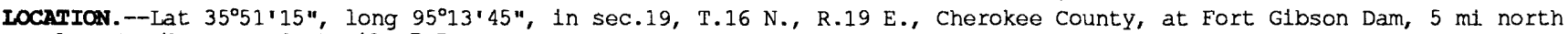
of Fort Gibson, and at mile 7.7 .

DRATIAGE AREA. --12,492 $\mathrm{mi}^{2}$.

Monthly and yearly discharge

\begin{tabular}{lrcc}
\hline Month & $\begin{array}{c}\text { Total } \\
\left(\mathrm{ft}^{3} / \mathrm{s}\right)\end{array}$ & $\begin{array}{c}\text { Mean } \\
\left(\mathrm{ft}^{3} / \mathrm{s}\right)\end{array}$ & $\begin{array}{c}\text { Runoff } \\
(\mathrm{acre-feet)}\end{array}$ \\
\hline October & 57,192 & 1,844 & 113,400 \\
November & 783,478 & 26,116 & $1,554,000$ \\
December & 228,992 & 7,387 & 454,200 \\
January & 272,378 & 8,786 & 540,200 \\
February & 158,672 & 5,667 & 314,700 \\
March & 246,300 & 7,945 & 488,500 \\
April & 376,173 & 12,539 & 746,100 \\
May & $1,346,980$ & 43,451 & $2,672,000$ \\
June & $1,396,267$ & 46,542 & $2,769,000$ \\
July & 833,028 & 26,872 & $1,652,000$ \\
August & 188,330 & 6,075 & 373,500 \\
September & 19,643 & 655 & 38,980 \\
\hline Water year 1995 & $5,907,433$ & 16,185 & $11,720,000$ \\
\hline
\end{tabular}




\section{ARKANSAS RIVER NEAR MOSKOGEE, OKLABOMA}

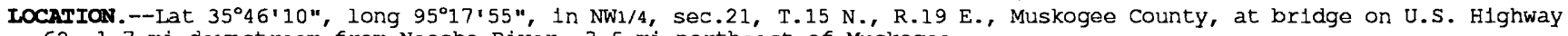
$62,1.7 \mathrm{mi}$ downstream from Neosho River, $3.5 \mathrm{mi}$ northeast of Muskogee.

DRAnNae AREA.--96,674 $\mathrm{mi}^{2}$ of which 12,541 $\mathrm{mi}^{2}$ probably is noncontributing.

Rararks.--Gaging station discontinued Sept. 30, 1970, due to backwater conditions. Streamflow computed by combining flow at station 07165570 Arkansas River near Haskell, station 07176000 Verdigris River near Claremore, station 07178200 Bird Creek at State Highway 266 near Catoosa, station 07193000 Fort Gibson Lake Discharge near Fort Gibson, and estimating the flow for the ungaged intervening drainage area.

Monthly and yearly discharge

\begin{tabular}{lrr}
\hline \multicolumn{1}{c}{ Month } & $\begin{array}{c}\text { Mean } \\
\left(\mathrm{ft}^{3} / \mathrm{s}\right)\end{array}$ & $\begin{array}{c}\text { Runoff } \\
\text { (acre-feet) }\end{array}$ \\
\hline October & 4,407 & 271,000 \\
November & 46,347 & $2,758,000$ \\
December & 20,151 & $1,239,000$ \\
January & 13,820 & 849,800 \\
February & 10,026 & 556,800 \\
March & 32,274 & $1,984,000$ \\
April & 32,495 & $1,934,000$ \\
May & 107,015 & $6,580,000$ \\
June & 161,550 & $9,613,000$ \\
July & 86,647 & $5,328,000$ \\
August & 46,796 & $2,877,000$ \\
September & 10,115 & 601,900 \\
& & $34,590,000$ \\
\hline Water year 1995 & 47,782 & \\
\hline
\end{tabular}


Iocarrow.--Lat $36^{\circ} 06^{\prime} 11^{\prime}$, long $94^{\circ} 20^{\prime} 39^{\prime \prime}$, in NWl/4SEl/4 sec.36, T.17 N., R.32 W., Washington County, Hydrologic Unit

11110103, on left bank at downstream side of state Highway 16 bridge, at Savoy.

DRATAGE AREA.--167 $\mathrm{mi}^{2}$.

AVERAGE DISCARRES.--2 years (water years 1980,86 ) $105 \mathrm{ft}^{3} / \mathrm{s}$.

ExurRariss.--April 1979 to December 1981, October 1985 to september 1986, August to september 1995: maximum discharge $9,530 \mathrm{ft}^{3} / \mathrm{s}$ Nov. 19, 1985; minimum $1.6 \mathrm{ft}^{3} / \mathrm{s}$ Aug. 11, 1980.

RomRrs.--Water discharge records good. Satellite telemeter at station.

Monthly and yearly discharge

\begin{tabular}{|c|c|c|c|c|c|}
\hline Month & $\begin{array}{l}\text { Total } \\
\left(\mathrm{ft}^{3} / \mathrm{s}\right)\end{array}$ & $\begin{array}{l}\text { Maximum } \\
\text { daily } \\
\left(\mathrm{ft}^{3} / \mathrm{s}\right)\end{array}$ & $\begin{array}{l}\text { Minimum } \\
\mathrm{dail}^{2} \\
\left(\mathrm{ft}^{3} / \mathrm{s}\right)\end{array}$ & $\begin{array}{c}\text { Mean } \\
\left(\mathrm{ft}^{3} / \mathrm{s}\right)\end{array}$ & $\begin{array}{c}\text { Runoff } \\
\text { (acre-feet) }\end{array}$ \\
\hline October & --- & --- & --- & --- & --- \\
\hline November & --- & --- & --- & --- & --- \\
\hline December & --- & --- & --- & --- & --- \\
\hline January & --- & --- & --- & --- & -- \\
\hline February & --- & --- & --- & --- & --- \\
\hline March & --- & --- & --- & --- & --- \\
\hline April & --- & --- & --- & --- & --- \\
\hline May & --- & --- & --- & --- & -- \\
\hline June & --- & --- & --- & --- & --- \\
\hline July & --- & --- & --- & --- & --- \\
\hline August & --- & --- & --- & --- & --- \\
\hline September & 345.9 & 24 & 8.3 & 11.5 & 686 \\
\hline Water year 1995 & -- & -- & -- & --- & --- \\
\hline
\end{tabular}


PERIOD OF RECORD.--April 1974 to current year.

REaRks.--Specific conductance, pH, water temperature, dissolved oxygen, and alkalinity were determined in the field. WATER-QUALITY DATA, WATER YEAR OCTOBER 1994 TO SEPTEMBER 1995

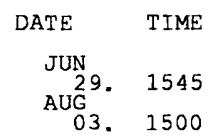

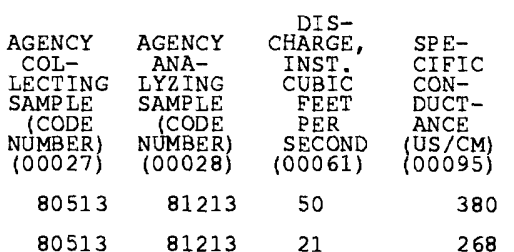

PH
WATER

WHOLE

FIELD

ARD

UNITS)
$(00400)$$$
\text { (00 }
$$

TEMPER-
ATURE
WATER
(DEG C)

WATER DIS-

DEG C) SMG/L

8.0

22.5

27.5

$(00300)$

OXYGEN, SOLVED (PERSENT ATION

8.2

$8.5 \quad 102$

COLIFECAL, 0.7 UM-MF (COLS. $100 \mathrm{ML}$

$80513 \quad 81213$
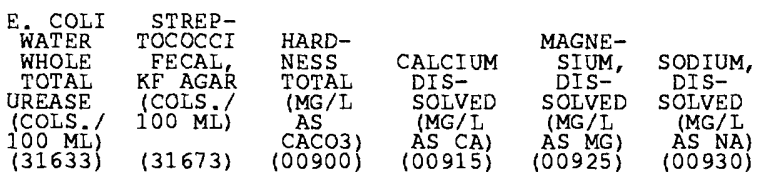

9.6

124

240

$<3$

(00925)

AS NA)
$(00930)$
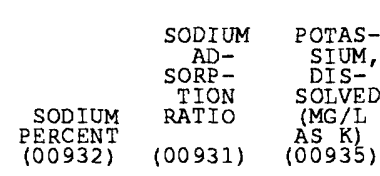
TION RATIO (00931)

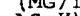
(MG/L

$$
110 \quad 41
$$
2.2
6.3
4.9
0.
2.6
8
$0.2 \quad 3.3$

2.3
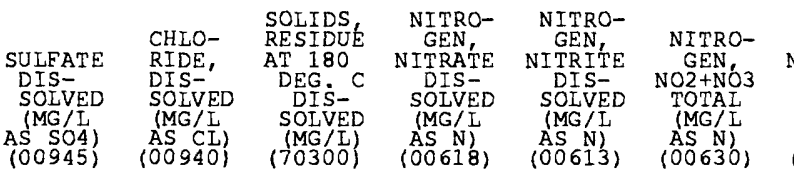

NITRO- NITRO-

$\begin{array}{llllllll}7.9 & 6.1 & 150 & 1.79 & 0.010 & 1.80 & 1.80 & 0.010 \\ 6.6 & 7.2 & 144 & 1.59 & 0.010 & 1.60 & 1.60 & 0.020\end{array}$
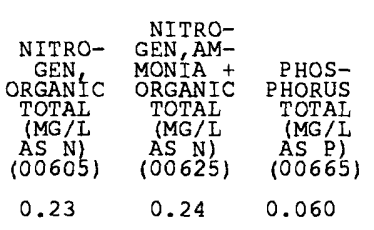

PHOS- PHOS-

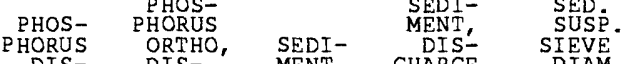

DIS- DIS- MENT, CHARGE, SIEVE

(MG/L SOVED SUS- SUS S PUS FINER

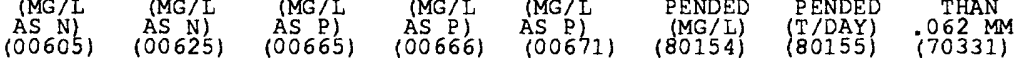

0.23

0.24

0.050

0.020

33

4.5

95

98 


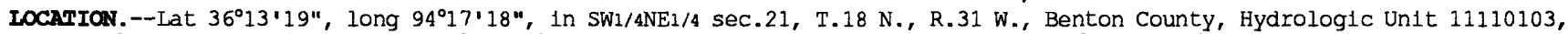
on left bank $0.7 \mathrm{mt}$ downstream from Little osage Creek, and $3.2 \mathrm{ml}$ northwest of Elm Springs.

DRATNGS AREA. $-130 \mathrm{mi}^{2}$.

AVERAGE DISCRARE: --25 years (water years 1951-75), $115 \mathrm{ft}^{3} / \mathrm{s}$.

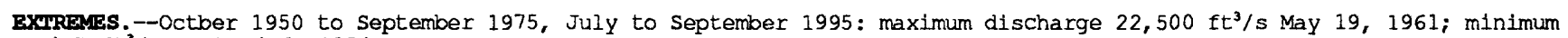
$4.7 \mathrm{ft}^{3} / \mathrm{s}$ Sept. 4-6, 1954 .

REMARKs.--Water-discharge records good. Low flow slightly regulated by operation of small lake at Cave springs, and northwest Arkansas sewage treatment plant. Satellite telemeter at station.

Monthly and yearly discharge

\begin{tabular}{|c|c|c|c|c|c|}
\hline Month & $\begin{array}{l}\text { Total } \\
\left(\mathrm{ft}^{3} / \mathrm{s}\right)\end{array}$ & $\begin{array}{c}\text { Maximum } \\
\mathrm{dail} \\
\left(\mathrm{ft}^{3} / \mathrm{s}\right)\end{array}$ & $\begin{array}{l}\text { Minimum } \\
\text { daily } \\
\left(\mathrm{ft}^{3} / \mathrm{s}\right)\end{array}$ & $\begin{array}{c}\text { Mean } \\
\left(\mathrm{ft}^{3} / \mathrm{s}\right)\end{array}$ & $\begin{array}{c}\text { Runoff } \\
\text { (acre-feet) }\end{array}$ \\
\hline October & --- & --- & --- & -- & -- \\
\hline November & -- & --- & -- & -- & --- \\
\hline December & --- & --- & --- & --- & --- \\
\hline January & --- & --- & -- & --- & --- \\
\hline February & --- & -- & -- & -- & -- \\
\hline March & --- & -- & --- & --- & -- \\
\hline April & -- & -- & -- & -- & -- \\
\hline May & -- & --- & --- & --- & --- \\
\hline June & -- & --- & --- & -- & -- \\
\hline July & --- & -- & --- & -- & -- \\
\hline August & 2,688 & 124 & 62 & 86.7 & 5,330 \\
\hline September & 2,380 & 215 & 53 & 79.3 & 4,720 \\
\hline Water year 1995 & -- & -- & --- & -- & -- \\
\hline
\end{tabular}


IIIINOIS RIVGR SUBBASIN

07195000 OSAGE CREEK NEAR EIM SPRINGS, ARTANSAS-CONTINUED

PERIOD OF RECORD.--April 1974 to current year.

RERRKs.--Specific conductance, pH, water temperature, dissolved oxygen, and alkalinity were determined in the field.

$$
\begin{array}{cc}
\text { DATE } & \text { TIME } \\
\text { JUN } & \\
\text { 29. } & 1330 \\
\text { AUG } 3 . & 1300 \\
& \\
& \\
& \\
& \\
\text { DATE } & \text { TIME } \\
\text { JUN } & \\
29 . & 1330 \\
\text { AUG } & \\
03 . & 1300
\end{array}
$$

DATE TIME

JUN 29.1330

03. 1300

DATE TIME

JU 29. 1330

03. 1300
WATER-QUALITY DATA, WATER YEAR OCTOBER 1994 TO SEPTEMBER 1995

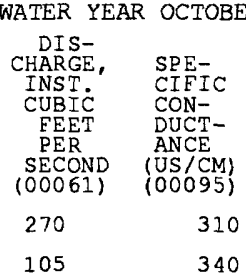

PH
WATER
WHOLE
FIELD
(STAND-

(STAND-
ARD
UNITS)

(ONITS)

TEMPER-
ATURE
WATER

(DEG C)

$$
8.1 \quad 18.5
$$

8. 223.0
340

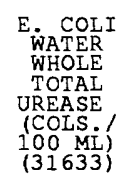

$$
\begin{aligned}
& \text { STREP- } \\
& \text { TOCOCCI } \\
& \text { FECAL } \\
& \text { KE AGAR }
\end{aligned}
$$

$100 \mathrm{ML}$ )

(31673)
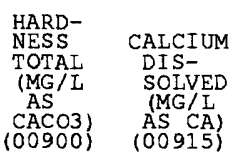

$\begin{array}{cc}\text { MAGNE- } & \\ \text { SIUM, } & \text { SODIUM, } \\ \text { DIS- } & \text { DIS- } \\ \text { SOLVED } & \text { SOLVED } \\ \text { (MG/L } & \text { (MG/L } \\ \text { AS MG) } & \text { AS NA) } \\ (00925) & (00930)\end{array}$

OXYGEN,

SOLVED

(MG/L)
$(00300)$

XXYGEN,
DIS--
SOLVED
(PER-
CENT
SATUR-

ATION)

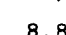

8.8

8.2

98

100

COLI-

FECAL

0.7

UM-MF,

(COLS.
$100 \mathrm{ML}\}$
$(31625)$

290

230

220

190

$(00900)$

AS CA)

$1.7 \quad 15$

$\begin{array}{lll}50 & 1.8 & 18\end{array}$

13050

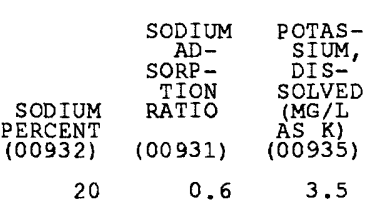

SOLIDS, NITRO-

NITRO- NITRO-
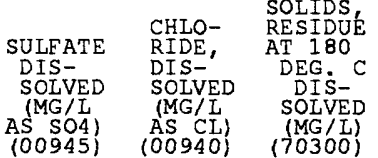
NITRA IE

NITRO-
GEN,
NITRITE
DIS-
SOIVED
(MG I
AS N)

SOLVED

(MG/L
AS N)
$(00618)$

AS N)
(00613)

4.49

0.010

3.59

0.010

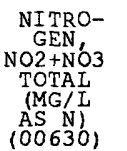

0.7

4.5

20

222

4.50

3.60

NITRO-

NITROGEN, GEN,

DIS-

SOLVED SOLVED

(MG/L (MG/L

AS N), AS N)
$(00631) \quad(00608)$

$\begin{array}{lll}15 & 20 & 222\end{array}$

4.50

0.010

3.60

0.010

PHOS-
PHORUS
DIS-
SOLVED
(MG/
AS P)

PHOS-

PHORUS

ORTHO,

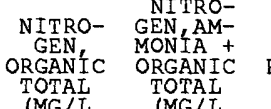

PHOS-

HORUS

(MG/L

(MG/L (MG/L

AS N N)
$(00605)$

AS N)
$(00625)$

$(00665)$

AS P)

SOLVED

(MG/I

AS P)
$(00671)$

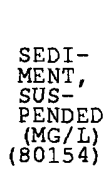

$\begin{array}{llllllll}0.21 & 0.22 & 0.400 & 0.380 & 0.400 & 32 & 23 & 92 \\ 0.19 & 0.20 & 0.740 & 0.720 & 0.760 & 14 & 4.0 & 99\end{array}$




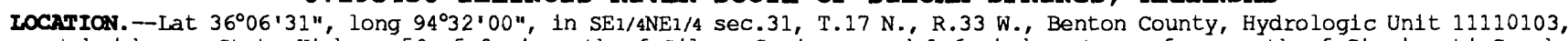
at bridge on state Highway $59,5.0 \mathrm{mi}$ south of siloam Springs, and $0.6 \mathrm{mi}$ downstream from mouth of Cincinnati Creek. DRATNACE AREA. $--575 \mathrm{mi}^{2}$.

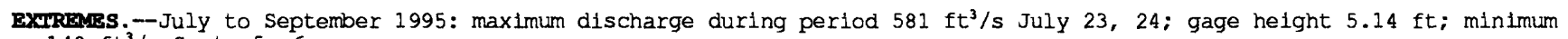
$148 \mathrm{ft}^{3} / \mathrm{s}$ Sept. 5,6 .

REMRKs.-Water-discharge records good. Satellite telemeter at station.

Monthly and yearly discharge

\begin{tabular}{|c|c|c|c|c|c|}
\hline Month & $\begin{array}{l}\text { Total } \\
\left(\mathrm{ft}^{3} / \mathrm{s}\right)\end{array}$ & $\begin{array}{l}\text { Maximum } \\
\text { daily } \\
\left(\mathrm{ft}^{3} / \mathrm{s}\right)\end{array}$ & $\begin{array}{l}\text { Minimum } \\
\text { daily } \\
\left(\mathrm{ft}^{3} / \mathrm{s}\right)\end{array}$ & $\begin{array}{c}\text { Mean } \\
\left(\mathrm{ft}^{3} / \mathrm{s}\right)\end{array}$ & $\begin{array}{c}\text { Runoff } \\
\text { (acre-feet) }\end{array}$ \\
\hline October & --- & --- & -- & --- & --- \\
\hline November & -- & --- & -- & --- & --- \\
\hline December & --- & --- & --- & --- & --- \\
\hline January & --- & --- & --- & -- & --- \\
\hline February & --- & --- & --- & --- & --- \\
\hline March & --- & -- & --- & -- & --- \\
\hline April & --- & --- & -- & --- & --- \\
\hline May & --- & --- & -- & -- & --- \\
\hline June & --- & --- & -- & --- & --- \\
\hline July & --- & --- & -- & $-\cdots$ & -- \\
\hline August & 6,572 & 293 & 162 & 212 & 13,040 \\
\hline September & 5,466 & 410 & 150 & 182 & 10,840 \\
\hline Water year 1995 & -- & -- & $-\cdots$ & $-\cdots$ & -- \\
\hline
\end{tabular}


PERIOD OF RECORD.--June 1995 to current year.

REMARKs.--Specific conductance, pH, water temperature, dissolved oxygen, and alkalinity were determined in the fleld. WATER-QUALITY DATA, WATER YEAR OCTOBER 1994 TO SEPTEMBER 1995

$$
\begin{array}{cc}
\text { DATE } & \text { TIME } \\
\text { JUN } & \\
29 . & 0800 \\
\text { AUG } & \\
03 . & 0830
\end{array}
$$

$$
\begin{aligned}
& \text { DATE TIME } \\
& \begin{array}{l}
\text { JUN } \\
\text { 29. } 0800
\end{array} \\
& 03.0830
\end{aligned}
$$

$$
\begin{array}{cc}
\text { DATE } & \text { TIME } \\
\text { JUN } & \\
29 . & 0800 \\
\text { AUG } & \\
03 . & 0830
\end{array}
$$

DATE TIME

IUN 29.0800

A3. 0830

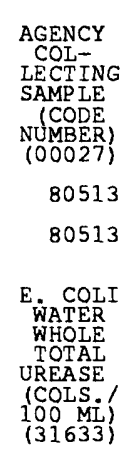

AGENCY

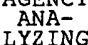

LYZING

(CODE)

NUMBER)
(00028)

81213

81213

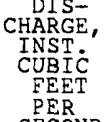

SPE-
CIFIC
CON-
DUCT-
ANCE
(US/CM)
$00095)$

PH
WATER
WHOLE
FIELD
(STAND-
ARD
UNITS)
(00400)

ATURE

WATER

SECOND
(00061)

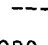

250

288

7.4
7.9

21.0

24.5

OXYGEN,
DIS-
SOIVED

SOLVED

$100300)$

6.5

6.4

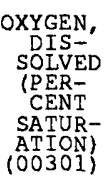

COLI-

FORM 0.7 UM-MF (COLS, (31625)
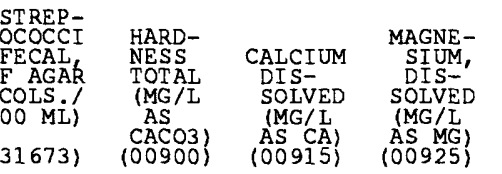$$
19.5
$$$$
\text { 6. } 4
$$

76

$$
100
$$

50

SODIUM,
DIS-
SOLVED
(MG/L
AS NA)
$(00930)$
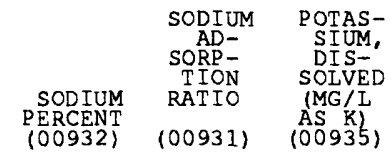

$\begin{array}{ll}110 & 43 \\ 120 & 44\end{array}$

$\begin{array}{cc}1.8 & 13 \\ 1.9 & 9.2\end{array}$

$\begin{array}{rr}40 & 92\end{array}$

19

$$
\begin{aligned}
& 0.5 \\
& 0.4
\end{aligned}
$$$$
3.0
$$

3.4
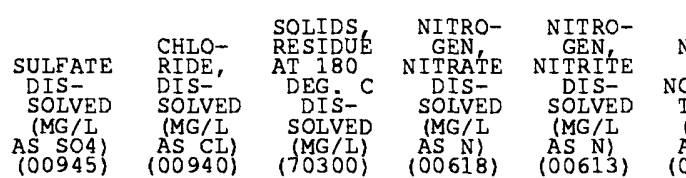

8.3

$$
9.3
$$

8.5 11

162

166

2.79

2.29

0.010

0.010

PHOS-
PHOS-
PHORUS
ORORUS

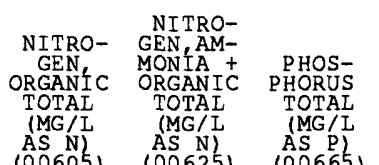

DIS-

SOLVED

(MG / L )

DOIS-

AS P )

$(00666)$
NITRO-

$\begin{array}{cc}\text { NITRO- } & \text { NITRO- } \\ \text { GEN } & \text { GEN } \\ \text { NO2+NO3 } & \text { AMMONIA }\end{array}$

NO2+NO3 AMMONIA

$\begin{array}{ll}\text { DIS- } & \text { DIS- } \\ \text { SOLVED } & \text { SOIVED } \\ \text { (MG/I } & \text { (MG/L }\end{array}$

$\begin{array}{ccc}\text { AS N) } & A S N) & \text { AS N } \\ (00630) & (00631) & (00608)\end{array}$

$$
\begin{array}{lll}
2.80 & 2.80 & 0.010
\end{array}
$$

$\begin{array}{lll}2.30 & 2.30 \quad 0.010\end{array}$$$
0.21
$$$$
0.22
$$$$
0.170
$$$$
0.110
$$$$
0.120
$$

0.190

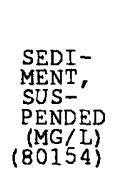

SEDI-
MENT,
DIS-
CHARGE,
SUS-
PENDED
\{T/DAY
80155

SED.
SUSP.
SIEVE
DIAM.
FINER
THAN
T62 MM
(70331)

$\begin{array}{llll}0.21 & 0.22 & 0.170 & 0.110 \\ 0.19 & 0.20 & 0.190 & 0.190\end{array}$

0.190

$\begin{array}{ll}59 & --- \\ 48 & 36\end{array}$

99

93 
IOCArION. - Lat $36^{\circ} 07^{\prime} 48^{\prime \prime}$, long $94^{\circ} 34^{\prime} 19^{\prime \prime}$, in NW1/4NE1/4, sec.18, T.19 N., R.26 E., Adair County, near right bank on downstream side of bridge on U.S. Highway $59,1.5 \mathrm{mi}$ north of Watts, $4.5 \mathrm{mi}$ downstream from Cincinnati Creek, and at mile 106.2 .

DRATIRE AREA. $--635 \mathrm{mi}^{2}$.

AVERAGE DISChARGE. -40 years, $632 \mathrm{ft}^{3} / \mathrm{s}$.

Exurmas.--August 1955 to current year: Maximum discharge $68,000 \mathrm{ft}^{3} / \mathrm{s}$ July 25, $1960 ; \mathrm{minimum} 8.6 \mathrm{ft}{ }^{3} / \mathrm{s}$ Oct. 26 , 1955 , Sept. 19, Oct. 14, 1956 .

RERRRs.--Records good. Since July 2, 1957, small diversion above station for municipal water supply for city of siloam Springs, Arkansas. Satellite telemeter at station.

Monthly and yearly discharge

\begin{tabular}{|c|c|c|c|c|c|}
\hline Month & $\begin{array}{l}\text { Total } \\
\left(\mathrm{ft}^{3} / \mathrm{s}\right)\end{array}$ & $\begin{array}{c}\text { Maximum } \\
\mathrm{daily} \\
\left(\mathrm{ft}^{3} / \mathrm{s}\right)\end{array}$ & $\begin{array}{l}\text { Minimum } \\
\mathrm{daily} \\
\left(\mathrm{ft}^{3} / \mathrm{s}\right)\end{array}$ & $\begin{array}{c}\text { Mean } \\
\left(\mathrm{ft}^{3} / \mathrm{s}\right)\end{array}$ & $\begin{array}{c}\text { Runoff } \\
\text { (acre-feet) }\end{array}$ \\
\hline October & 5,198 & 351 & 113 & 168 & 10,310 \\
\hline November & 33,950 & 6,090 & 153 & 1,132 & 67,340 \\
\hline December & 19,511 & 3,360 & 301 & 629 & 38,700 \\
\hline January & 46,019 & 10,200 & 283 & 1,484 & 91,280 \\
\hline February & 14,890 & 915 & 361 & 532 & 29,530 \\
\hline March & 26,456 & 4,090 & 414 & 853 & 52,480 \\
\hline April & 38,870 & 7,750 & 327 & 1,296 & 77,100 \\
\hline May & 66,324 & 10,200 & 650 & 2,139 & 131,600 \\
\hline June & 48,460 & 10,500 & 523 & 1,615 & 96,120 \\
\hline July & 12,891 & 577 & 306 & 416 & 25,570 \\
\hline August & 6,787 & 299 & 163 & 219 & 13,460 \\
\hline September & 5,508 & 377 & 149 & 184 & 10,930 \\
\hline Water year 1995 & 324,864 & 10,500 & 113 & 890 & 644,400 \\
\hline
\end{tabular}


07195500 IILINOIS RIVER NEAR NATTS, OKLAEOMA--CONTINUED

PERIOD OF RECORD.--October 1989 to current year.

REMARKS.--Specific conductance, pH, water temperature, dissolved oxygen, and alkalinity were determined 1n the field. WATER-QUALITY DATA, WATER YEAR OCTOBER 1994 TO SEPTEMBER 1995

\begin{tabular}{|c|c|c|c|c|c|c|c|c|c|c|c|c|c|c|c|c|}
\hline \multirow{2}{*}{$\begin{array}{l}\text { DATE } \\
\begin{array}{l}\text { OCT } \\
27 \ldots \\
27 \ldots \\
27 \ldots \\
27 \ldots \\
27 \ldots \\
27 \ldots \\
27 \ldots \\
27 \ldots \\
27 \ldots \\
27 \ldots\end{array}\end{array}$} & \multicolumn{2}{|c|}{$\begin{array}{l}\text { SAMPIE } \\
\text { LOC- } \\
\text { ATION, } \\
\text { CROSS } \\
\text { SECTION } \\
\text { (FT EM } \\
\text { L BANK) } \\
(00009)\end{array}$} & \multicolumn{3}{|c|}{$\begin{array}{l}\text { BARO- } \\
\text { METRIC } \\
\text { PRES- } \\
\text { SURE } \\
\text { (MM } \\
\text { OE } \\
\text { HG) } \\
(00025)\end{array}$} & \multicolumn{6}{|c|}{$\begin{array}{l}\text { DIS- } \\
\text { CHARGE, } \\
\text { INST': } \\
\text { CUBIC } \\
\text { FEET } \\
\text { PER } \\
\text { SECOND } \\
(00061)\end{array}$} & \multicolumn{2}{|c|}{$\begin{array}{c}\text { GAGE } \\
\text { HEIGHT } \\
(\text { EET) } \\
(00065)\end{array}$} & $\begin{array}{l}\text { SPE- } \\
\text { CIFIC } \\
\text { CON- } \\
\text { DUCT- } \\
\text { ANCE } \\
\text { (US/CM) } \\
(00095)\end{array}$ & $\begin{array}{c}\text { OXYGEN, } \\
\text { DIS- } \\
\text { SOLVED } \\
(M G / L) \\
(00300)\end{array}$ & $\begin{array}{l}\text { PH } \\
\text { WATER } \\
\text { WHOLE } \\
\text { FIELD } \\
\text { (STAND- } \\
\text { ARD } \\
\text { UNITS) } \\
(00400)\end{array}$ \\
\hline & $\begin{array}{l}1100 \\
1101 \\
1102 \\
1103 \\
1104 \\
1105 \\
1106 \\
1107 \\
1108 \\
1109 \\
1110\end{array}$ & $\begin{array}{r}8.00 \\
18.0 \\
28.0 \\
38.0 \\
48.0 \\
58.0 \\
68.0 \\
78.0 \\
88.0 \\
98.0 \\
108\end{array}$ & $\begin{array}{l}14 \\
14 \\
14 \\
14 \\
14 \\
14 \\
14 \\
14 \\
14 \\
14 \\
14 .\end{array}$ & & $\begin{array}{l}751 \\
751 \\
751 \\
751 \\
751 \\
751 \\
751 \\
751 \\
751 \\
751 \\
751\end{array}$ & $\begin{array}{l}1028 \\
1028 \\
1028 \\
1028 \\
1028 \\
1028 \\
1028 \\
1028 \\
1028 \\
1028 \\
1028\end{array}$ & & $\begin{array}{l}1028 \\
1028 \\
1028 \\
1028 \\
1028 \\
1028 \\
1028 \\
1028 \\
1028 \\
1028 \\
1028\end{array}$ & & $\begin{array}{l}169 \\
169 \\
169 \\
169 \\
169 \\
169 \\
169 \\
169 \\
169 \\
169 \\
169\end{array}$ & & $\begin{array}{l}2 . \\
2 . \\
2 . \\
2 . \\
2 . \\
2 . \\
2 . \\
2 . \\
2 . \\
2 . \\
2 .\end{array}$ & $\begin{array}{l}320 \\
32 \\
320 \\
329 \\
329 \\
329 \\
329 \\
329 \\
320 \\
329 \\
329\end{array}$ & $\begin{array}{l}9 \\
9 \\
9 \\
9 \\
9 \\
9 \\
9 \\
9 \\
9 \\
9 \\
9\end{array}$ & $\begin{array}{l}10.1 \\
10: 1 \\
10: 1 \\
10: 0 \\
10: 1 \\
10: 1 \\
10: 1 \\
10: 1 \\
10: 1 \\
10.0 \\
10.0\end{array}$ & $\begin{array}{l}8.1 \\
8: 1 \\
8: 1 \\
8: 1 \\
8: 1 \\
8: 1 \\
8: 1 \\
8: 1 \\
8: 1 \\
8: 1 \\
8.1\end{array}$ \\
\hline DATE & TIME & $\begin{array}{l}\text { AGENCY } \\
\text { COL- } \\
\text { LECTING } \\
\text { SAMPLE } \\
\text { (CODE } \\
\text { NUMBER) } \\
(00027)\end{array}$ & $\begin{array}{l}\text { AGENCY } \\
\text { ANA- } \\
\text { LYZING } \\
\text { SAMPLE } \\
\text { (CODE } \\
\text { NUMBER } \\
(00028)\end{array}$ & $\begin{array}{c}\text { DI } \\
\text { CHA } \\
\text { IN } \\
C U \\
F E \\
P \\
\text { SEC } \\
100\end{array}$ & $\begin{array}{l}\text { IS - } \\
\text { IRGE, } \\
\text { JST. } \\
\text { JBIC } \\
\text { EET } \\
\text { ER } \\
\text { COND } \\
0611\end{array}$ & $\begin{array}{c}\text { SPE- } \\
\text { CIFIC } \\
\text { CON- } \\
\text { DUCT- } \\
\text { ANCE } \\
\{\text { US/CM } \\
\{00095\}\end{array}$ & $\begin{array}{c}\text { } \\
\text { WA } \\
\text { WH } \\
\text { FI } \\
\text { (ST } \\
\text { A } \\
\text { UNI } \\
100\end{array}$ & $\begin{array}{l}\text { PH } \\
\text { TER } \\
\text { OLE } \\
\text { ELD } \\
\text { AND- } \\
\text { RD } \\
\text { [TS } \\
400 \text { ) }\end{array}$ & $\begin{array}{r}\text { TEM } \\
\text { AT } \\
\text { A } \\
(D E \\
(00\end{array}$ & $\begin{array}{l}\text { RE- } \\
\text { Re } \\
\text { C) } \\
20)\end{array}$ & $\begin{array}{l}\text { TEMP } \\
\text { ATU } \\
\text { WAT } \\
\text { DEEG } \\
000\end{array}$ & $\begin{array}{l}\text { R- } \\
\text { E } \\
\text { R } \\
\text { C) } \\
0\end{array}$ & $\begin{array}{l}\text { TUR- } \\
\text { BID- } \\
\text { ITY } \\
(\text { NTU) } \\
(00076)\end{array}$ & $\begin{array}{l}\text { BARO- } \\
\text { METRIC } \\
\text { PRES- } \\
\text { SURE } \\
\text { (MM } \\
\text { OF } \\
\text { HG) } \\
(00025)\end{array}$ & $\begin{array}{c}\text { OXYGEN, } \\
\text { DIS- } \\
\text { SOLVED } \\
(M G / L) \\
(00300)\end{array}$ & $\begin{array}{l}\text { OXYGEN, } \\
\text { DIS- } \\
\text { SOLVED } \\
\text { (PER- } \\
\text { CENT } \\
\text { SATUR- } \\
\text { ATION) } \\
\text { (O0301) }\end{array}$ \\
\hline $27 \ldots$ & 1200 & 1028 & 80020 & & 169 & 329 & 8. & 1 & & .0 & 14 & & -- & 751 & 10.1 & 100 \\
\hline $20 \ldots$ & 1700 & 1028 & 80020 & & 449 & 275 & 8. & 0 & & .0 & 10 & & 3.3 & 748 & 10.8 & 98 \\
\hline $16 \ldots$ & 1600 & 1028 & 80020 & & 531 & 288 & 8. & 1 & & .0 & & .5 & --- & 755 & 12.5 & 105 \\
\hline $09 .$. & 1000 & 1028 & 80020 & & 200 & 144 & 7. & 8 & & .0 & 16 & & 41 & 740 & 8.8 & 92 \\
\hline $28 \ldots$ & 1700 & 1028 & 80020 & & 519 & 265 & 7. & 9 & & .0 & 22 & & 13 & 750 & 8.6 & 101 \\
\hline $31 \ldots$ & 1430 & 1028 & 80020 & & 292 & 288 & 7. & 9 & & .5 & 27 & & -- & 750 & 8.0 & 103 \\
\hline DATE & $\begin{array}{l}\text { NITRO- } \\
\text { GEN } \\
\text { DIS- } \\
\text { SOLVED } \\
\text { (MG /L } \\
\text { AS N) } \\
(00602)\end{array}$ & $\begin{array}{c}\text { HARD- } \\
\text { NESS } \\
\text { TOTAL } \\
\text { (MG/L } \\
\text { AS } \\
\text { CACO3) } \\
(00900)\end{array}$ & $\begin{array}{l}\text { HARD- } \\
\text { NESS } \\
\text { NONCARB } \\
\text { DISSOLV } \\
\text { FID. AS } \\
\text { CACO3 } \\
(M G / L) \\
(00904)\end{array}$ & $\begin{array}{l}\text { CAL } \\
\text { DI } \\
\text { SOI } \\
\text { (M } \\
\text { AS } \\
100\end{array}$ & $\begin{array}{l}\text { CIUM } \\
\text { IS- } \\
\text { LVED } \\
\text { (G/L } \\
\text { CA) } \\
9151\end{array}$ & $\begin{array}{l}\text { MAGNE- } \\
\text { SIUM, } \\
\text { DIS- } \\
\text { SOLVED } \\
\text { (MG/L } \\
\text { AS MG) } \\
(00925)\end{array}$ & $\begin{array}{l}\text { SOD } \\
\text { DI } \\
\text { SOI } \\
(M \\
\text { AS } \\
100\end{array}$ & $\begin{array}{l}\text { IUM, } \\
\text { S- } \\
\text { UVED } \\
\text { G/L } \\
\text { NA) } \\
930 \text { ) }\end{array}$ & $\begin{array}{l}\text { SOD } \\
\text { PER } \\
100\end{array}$ & $\begin{array}{l}\mathrm{UM} \\
21 \\
21\end{array}$ & $\begin{array}{r}\text { SODI } \\
\text { AD } \\
\text { SOR } \\
\text { TIC } \\
\text { RAT } \\
1009\end{array}$ & $\begin{array}{l}\text { UM } \\
\text { P- } \\
\text { IO } \\
\text { I1) }\end{array}$ & $\begin{array}{l}\text { POTAS- } \\
\text { SIUM, } \\
\text { DIS- } \\
\text { SOLVED } \\
\text { (MG/L } \\
\text { AS K) } \\
(00935)\end{array}$ & $\begin{array}{l}\text { BICAR- } \\
\text { BONATE } \\
\text { WATER } \\
\text { DIS IT } \\
\text { FIEID } \\
\text { MG/L AS } \\
\text { HCO3 } \\
(00453)\end{array}$ & $\begin{array}{c}\text { CAR- } \\
\text { BONATE } \\
\text { WATER } \\
\text { DIS IT } \\
\text { FIELD } \\
\text { MG/L AS } \\
\text { CO3 } \\
(00452)\end{array}$ & $\begin{array}{l}\text { ALKA- } \\
\text { LINITY } \\
\text { WAT DIS } \\
\text { TOT IT } \\
\text { FIELD } \\
\text { MG/L AS } \\
\text { CACO3 } \\
(39086)\end{array}$ \\
\hline $27 \ldots$ & -- & -- & -- & & --- & -- & -- & & $-\cdots$ & & $-\cdots$ & & --- & 129 & 0 & 106 \\
\hline $20 \ldots$ & $-\cdots$ & 120 & 12 & & 43 & 1.9 & 7. & 0 & 11 & & & 3 & 2.3 & 126 & 0 & 103 \\
\hline $16 \dot{Y}^{\circ}$ & -- & $-\cdots$ & --- & & --- & -- & -- & & -- & & $-\cdots$ & & -- & 114 & 0 & 94 \\
\hline $09 .$. & 1.6 & 62 & 12 & & 22 & 1.6 & 2. & 8 & 8 & & & 2 & 3.3 & 60 & 0 & 49 \\
\hline $28 \ldots$ & $\cdots$ & 110 & 0 & & 42 & 1.8 & 6. & 8 & 11 & & & .3 & 2.8 & 137 & 0 & 112 \\
\hline & -- & $-\infty$ & $-\cdots$ & & --- & -- & -- & & $\cdots$ & & $-\cdots$ & & -- & 125 & 0 & 103 \\
\hline DATE & $\begin{array}{l}\text { SULFATE } \\
\text { DIS- } \\
\text { SOLVED } \\
\text { (MG/I } \\
\text { AS SO4) } \\
(00945)\end{array}$ & $\begin{array}{l}\text { CHLO- } \\
\text { RIDE, } \\
\text { DIS- } \\
\text { SOLVED } \\
\text { (MG/L } \\
\text { AS CL) } \\
(00940)\end{array}$ & $\begin{array}{l}\text { FLUO- } \\
\text { RIDE, } \\
\text { DIS- } \\
\text { SOLVED } \\
\text { (MG/L } \\
\text { AS F) } \\
(00950)\end{array}$ & & $\begin{array}{l}\text { LICA, } \\
\text { IS- } \\
\text { LVED } \\
\text { MG / } \\
\text { AS } \\
\text { IO2) } \\
09551\end{array}$ & $\begin{array}{l}\text { SOLIDS, } \\
\text { RESIDUE } \\
\text { AT } 180 \\
\text { DEG. C } \\
\text { DIS- } \\
\text { SOLVED } \\
(M G / L) \\
(70300)\end{array}$ & & $\begin{array}{l}\text { OLIDS, } \\
\text { UM OF } \\
\text { ONSTI- } \\
\text { UENTS, } \\
\text { DIS- } \\
\text { OLVED } \\
\text { MG/L) } \\
\text { T03011 }\end{array}$ & & $\begin{array}{l}\text { OLIDS, } \\
\text { DIS- } \\
\text { OLVED } \\
\text { (TONS } \\
\text { PER } \\
\text { C-FT) } \\
(0303 \text { ) }\end{array}$ & $\begin{array}{r}\text { SOI } \\
\text { D } \\
\text { SOI } \\
\text { (T } \\
\text { P } \\
\text { DI } \\
170\end{array}$ & $\begin{array}{l}\text { DS, } \\
\text { S- } \\
\text { VED } \\
\text { NS } \\
\text { R } \\
\text { Y) } \\
021\end{array}$ & $\begin{array}{l}\text { NITRO- } \\
\text { GEN, } \\
\text { NITRATE } \\
\text { TOTAL } \\
\text { (MG/I } \\
\text { AS N) } \\
(00620)\end{array}$ & $\begin{array}{c}\text { NITRO- } \\
\text { GEN, } \\
\text { NITRATE } \\
\text { DIS- } \\
\text { SOLVED } \\
\text { (MG/L } \\
\text { AS N) } \\
(00618)\end{array}$ & $\begin{array}{l}\text { NITRO- } \\
\text { GEN, } \\
\text { NITRATE } \\
\text { DIS- } \\
\text { SOLVED } \\
\text { (MG/L } \\
\text { AS NO3) } \\
(71851)\end{array}$ & $\begin{array}{l}\text { NITRO- } \\
\text { GEN, } \\
\text { NITRITE } \\
\text { DIS- } \\
\text { SOLVED } \\
\text { (MG/L } \\
\text { AS N) } \\
(00613)\end{array}$ \\
\hline $\begin{array}{l}O C T \\
27 .\end{array}$ & -- & $-\cdots$ & --- & & --- & $-\cdots$ & --- & & -- & & $-\cdots$ & & 1.40 & -- & -- & $<0.010$ \\
\hline $20 \ldots$ & 10 & 9.6 & $<0.10$ & & 6.7 & 160 & 154 & & & 22 & 194 & & 2.60 & --- & --- & $<0.010$ \\
\hline $16 \ldots$ & -- & $-\cdots$ & --- & & $-\cdots$ & $-\cdots$ & --- & & -- & & -- & & 3.00 & -- & --- & $<0.010$ \\
\hline $09 .$. & 5.5 & 3.1 & $<0.10$ & & 7.6 & 94 & 82 & & & 13 & 2590 & & 1.18 & 1.18 & 5.2 & 0.020 \\
\hline $28 \ldots$ & 6.8 & 8.6 & $<0.10$ & & 6.7 & 157 & 154 & & & 21 & 220 & & 2.50 & -- & --- & $<0.010$ \\
\hline $31 \ldots$ & -- & $-\cdots$ & -- & & --- & -- & --- & & -- & & -- & & 2.19 & 2.19 & 9.7 & 0.010 \\
\hline
\end{tabular}

\begin{tabular}{|c|c|c|c|c|c|c|c|c|c|c|c|}
\hline $\mathrm{TE}$ & $\begin{array}{l}\text { NITRO- } \\
\text { GENO- } \\
\text { GITIITE } \\
\text { DIS- } \\
\text { SOLVED } \\
\text { MGLI } \\
\text { AS NO2) } \\
(71856)\end{array}$ & $\begin{array}{l}\text { NITRO- } \\
\text { GEN } \\
\text { NO2+NO3 } \\
\text { TOTAL } \\
\text { MGG/L } \\
\text { AS N) } \\
(00630)\end{array}$ & $\begin{array}{c}\text { NITRO- } \\
\text { GEN } \\
\text { GENO3 } \\
\text { DIS } \\
\text { DOLVED } \\
\text { SOLEE } \\
\text { MGG NI } \\
\text { AS NI) } \\
(00631)\end{array}$ & $\begin{array}{l}\text { NITRO- } \\
\text { GEN } \\
\text { AMMONIA } \\
\text { DIS- } \\
\text { SOLVED } \\
\text { MGG LI } \\
\text { AS N) } \\
(00608)\end{array}$ & $\begin{array}{l}\text { NITRO- } \\
\text { GEN } \\
\text { GEN } \\
\text { AMONIA } \\
\text { DIS- } \\
\text { SOLVED } \\
\text { MG IIL } \\
\text { AS NH4) } \\
(71346)\end{array}$ & $\begin{array}{l}\text { NITRO- } \\
\text { GEN } \\
\text { GRANIC } \\
\text { TOTAL } \\
\text { TOTG } \\
\text { AS N N } \\
(00605)\end{array}$ & $\begin{array}{l}\text { NITRO- } \\
\text { GEN - } \\
\text { ORGANIC } \\
\text { DIS- } \\
\text { SOLVED } \\
\text { SMG/L } \\
\text { AS N) } \\
(00607)\end{array}$ & $\begin{array}{l}\text { NITRO- } \\
\text { GEN AMM- } \\
\text { MONIA } \\
\text { ORGANIC } \\
\text { TOTAL } \\
\text { TMG/L } \\
\text { AS N N) } \\
(00625)\end{array}$ & $\begin{array}{l}\text { NITRO- } \\
\text { GEN AM- } \\
\text { GONIA } \\
\text { MONGANIC } \\
\text { ORGA } \\
\text { DIS. } \\
\text { (MG L } \\
\text { AS N } \\
(00623) \\
(00623)\end{array}$ & $\begin{array}{c}\text { NITRO- } \\
\text { GEN, } \\
\text { TOTÁ } \\
\text { (MG/L } \\
\text { AS N) } \\
(00600)\end{array}$ & $\begin{array}{c}\text { PHOS- } \\
\text { PHORUS } \\
\text { TOTAL } \\
\text { (MG/L } \\
\text { AS P P) } \\
(00665)\end{array}$ \\
\hline & --- & 1.40 & 1.40 & 0.020 & 0.03 & - & --- & $<0.20$ & $<0.20$ & -- & 0.230 \\
\hline & - & 2.60 & 2.60 & $<0.015$ & -- & -- & --- & $<0.20$ & $<0.20$ & --- & 0.080 \\
\hline & -- & 3.00 & 3.00 & $<0.015$ & -- & -- & --- & $<0.20$ & $<0.20$ & --- & 0.170 \\
\hline & 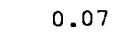 & 1.20 & 1.20 & 0.040 & 0.05 & 0.76 & 0.36 & 0.80 & 0.40 & 2.0 & 0.320 \\
\hline & 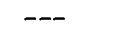 & 2.50 & 2.50 & 0.020 & 0.03 & 0.18 & --- & 0.20 & $<0.20$ & 2.7 & 0.110 \\
\hline & & 2.20 & 2.20 & .020 & 0.03 & 0.18 & -- & 0.20 & $<0.20$ & 2.4 & 0.150 \\
\hline
\end{tabular}


IILINOIS RTVER SUBBASIN

07195500 ILIINOIS RIVER NEAR WATTS, OKIAHOMA-CONTINOED

WATER-QUALITY DATA, WATER YEAR OCTOBER 1994 TO SEPTEMBER 1995

\begin{tabular}{|c|c|c|c|c|c|c|c|c|c|c|c|}
\hline DATE & $\begin{array}{l}\text { PHOS- } \\
\text { PHORUS } \\
\text { DIS- } \\
\text { SOLVED } \\
\text { (MG/L } \\
\text { AS P) } \\
(00666)\end{array}$ & $\begin{array}{l}\text { PHOS- } \\
\text { PHORUS } \\
\text { ORTHO, } \\
\text { DIS- } \\
\text { SOLVED } \\
\text { (MG/L } \\
\text { AS P) } \\
(00671)\end{array}$ & $\begin{array}{l}\text { PHOS- } \\
\text { PHATE, } \\
\text { ORTHO, } \\
\text { DIS- } \\
\text { SOLVED } \\
\text { (MG/L } \\
\text { AS PO4) } \\
(00660)\end{array}$ & $\begin{array}{c}\text { ARSENIC } \\
\text { DIS- } \\
\text { SOLVED } \\
\text { (UG/L } \\
\text { AS AS) } \\
(01000)\end{array}$ & $\begin{array}{c}\text { BARIUM, } \\
\text { DIS- } \\
\text { SOLVED } \\
\text { (UG/L } \\
\text { AS BA) } \\
(01005)\end{array}$ & $\begin{array}{l}\text { BERYL- } \\
\text { LIUM, } \\
\text { DIS- } \\
\text { SOLVED } \\
\text { (UG/L } \\
\text { AS BE) } \\
(01010)\end{array}$ & $\begin{array}{l}\text { BORON, } \\
\text { DIS- } \\
\text { SOLVED } \\
\text { (UG/I } \\
\text { AS B) } \\
(01020)\end{array}$ & $\begin{array}{l}\text { CADMIUM } \\
\text { DIS- } \\
\text { SOIVED } \\
\text { (UG/L } \\
\text { AS CD) } \\
(01025)\end{array}$ & $\begin{array}{l}\text { CHRO- } \\
\text { MIUM, } \\
\text { DIS- } \\
\text { SOLVED } \\
\text { (UG/L } \\
\text { AS CR) } \\
(01030)\end{array}$ & $\begin{array}{l}\text { COBALT, } \\
\text { DIS- } \\
\text { SOLVED } \\
\text { (UG/L } \\
\text { AS CO) } \\
(01035)\end{array}$ & $\begin{array}{c}\text { COPPER, } \\
\text { DIS- } \\
\text { SOLVED } \\
\text { (UG/L } \\
\text { AS CU) } \\
(01040)\end{array}$ \\
\hline \multirow{6}{*}{ 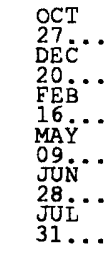 } & 0.190 & 0.200 & 0.61 & -- & -- & -- & -- & -- & -- & $\ldots$ & -- \\
\hline & 0.070 & 0.070 & 0.21 & $<1$ & 39 & $<0.5$ & 10 & $<1.0$ & $<5$ & $<3$ & $<10$ \\
\hline & 0.140 & 0.140 & 0.43 & $-\infty$ & -- & -- & --- & $-\cdots$ & --- & --- & --- \\
\hline & 0.180 & 0.170 & 0.52 & $<1$ & 36 & $<0.5$ & 10 & 3.0 & $<5$ & $<3$ & $<10$ \\
\hline & 0.090 & 0.100 & 0.31 & $<1$ & 49 & $<0.5$ & 40 & $<1.0$ & $<5$ & $<3$ & $<10$ \\
\hline & 0.150 & 0.150 & 0.46 & -- & -- & $-\cdots$ & --- & --- & --- & --- & --- \\
\hline DATE & $\begin{array}{l}\text { IRON, } \\
\text { DIS- } \\
\text { SOLVED } \\
\text { (UG/L } \\
\text { AS FE) } \\
(01046)\end{array}$ & $\begin{array}{l}\text { LEAD, } \\
\text { DIS- } \\
\text { SOLVED } \\
\text { (UG/L } \\
\text { AS PB) } \\
(01049)\end{array}$ & $\begin{array}{l}\text { LITHIUM } \\
\text { DIS- } \\
\text { SOLVED } \\
\text { (UG/L } \\
\text { AS LI) } \\
\text { (01130) }\end{array}$ & $\begin{array}{l}\text { MANGA- } \\
\text { NESE, } \\
\text { DIS- } \\
\text { SOIVED } \\
\text { (UG/I } \\
\text { AS MN) } \\
(01056)\end{array}$ & $\begin{array}{l}\text { MERCURY } \\
\text { DIS- } \\
\text { SOLVED } \\
\text { (UG/L } \\
\text { AS HG) } \\
\text { (71890) }\end{array}$ & $\begin{array}{l}\text { MOLYB- } \\
\text { DENUM, } \\
\text { DIS- } \\
\text { SOLVED } \\
\text { (UG/I } \\
\text { AS MO) } \\
(01060)\end{array}$ & $\begin{array}{l}\text { NICKEI, } \\
\text { DIS- } \\
\text { SOLVED } \\
\text { (UG /I } \\
\text { AS NI) } \\
(01065)\end{array}$ & $\begin{array}{l}\text { SILVER, } \\
\text { DIS- } \\
\text { SOLVED } \\
\text { (UG/I } \\
\text { AS AG) } \\
(01075)\end{array}$ & $\begin{array}{l}\text { STRON- } \\
\text { TIUM, } \\
\text { DIS- } \\
\text { SOLVED } \\
\text { (UG/L } \\
\text { AS SR) } \\
(01080)\end{array}$ & $\begin{array}{l}\text { VANA- } \\
\text { DIUM, } \\
\text { DIS- } \\
\text { SOLVED } \\
\text { (UG/L } \\
\text { AS V) } \\
(01085)\end{array}$ & $\begin{array}{l}\text { ZINC, } \\
\text { DIS- } \\
\text { SOLVED } \\
\text { (UG/I } \\
\text { AS ZN) } \\
(01090)\end{array}$ \\
\hline $27 \ldots$ & $-m$ & --- & $-\infty$ & $-\infty$ & $-\infty$ & $-\infty$ & -- & -- & -- & -- & -- \\
\hline $20 \ldots$ & 4 & $<10$ & $<4$ & 43 & $<0.1$ & $<10$ & $<10$ & $<1.0$ & 47 & $<6$ & $<3$ \\
\hline $16 \ldots$ & -- & --- & -- & --- & -- & --- & --- & -- & --- & -- & --- \\
\hline $09 \ldots$ & 170 & 20 & $<4$ & 17 & $<0.1$ & $<10$ & $<10$ & $<1.0$ & 34 & $<6$ & 5 \\
\hline JUI & 11 & $<10$ & $<4$ & 38 & $<0.1$ & $<10$ & $<10$ & $<1.0$ & 48 & $<6$ & 4 \\
\hline $31 \ldots$ & -- & --- & -- & -- & -- & -- & --- & -- & -- & -- & --- \\
\hline
\end{tabular}
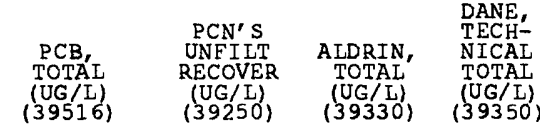

CHLOR-
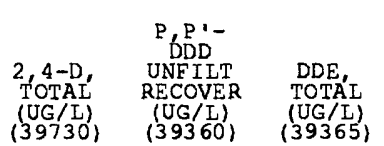

${ }^{\mathrm{P}} \mathrm{PDT}^{\mathrm{T}}-$

$\begin{array}{lll}\text { TOTAL } & \text { U-D, UNFILT } & \text { DDE, } \\ \text { RECOVER TOTAL RECOVER TOTAL } & \text { RECOVER }\end{array}$

(UG/L)

$(39730)$

( 39365 )

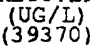

DEF

DII-

$\begin{array}{ll}\text { (UG/L) } & \text { (UG/L) } \\ (39040) & (39570)\end{array}$

MAY $\quad<0.100$

$<0.100$

$<0.010<0.100$

$<0.010$

0.030

$<0.010$

$<0.010$

$<0.010$

$<0.010$

0.010

$<0.100$

$<0.010<0.100$

$<0.010$

$<0.010<0.010$

$<0.010$

$<0.010$

$<0.010$

$<0.010$

DATE

$\begin{array}{cc} & \text { DISUL- } \\ \text { DI- } & \text { FOTON } \\ \text { ELDRIN } & \text { UNFIIT } \\ \text { TOTAL } & \text { RECOVER } \\ \text { (UG/L) } & \text { (UG/L) } \\ (39380) & (39011)\end{array}$

$\begin{array}{cc} & \text { ENDO- } \\ 2 \text { SULFAN, } \\ \text { TOTAL } & \text { I } \\ \text { TOTG } & \text { TOTAL } \\ (82183) & (\text { UG/L) } \\ (39388)\end{array}$

FONOFOS

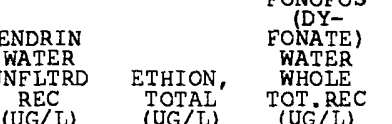

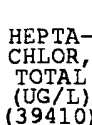

HEPTA-

CHLOR
EPOXIDE
TOTAL

TOTAL

(UG/L)
(39420)

IINDANE

TOTAL
(UG/L)
$(39340)$

MAIAA-

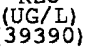

(39398)

(8. 010

$<0.010$

$<0.010$

$<0.010$

$(39530)$

$\operatorname{Mg}_{\text {MUN }}^{\operatorname{MAY}}<0.010<0.010$

$<0.010<0.010$

$<0.010$

$<0.010<0.010$

$<0.010$

$<0.010$

$<0.010$

$<0.010$

$<0.010$

DATE
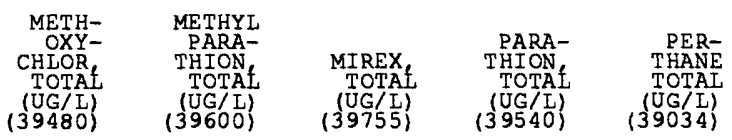

PHORATE
TOTAI
(UG/I)

SI LVEX,

( 39023$)$

(3976)

2, 4,5-T $(39740)$

TOX-
APHENE
TOTAL
TOTAL
$(3.5400)$

Totat
$(396 \mathrm{~L})$
$(3900)$

TOTAI

THION

MAY $\quad<0.010$

$<0.010$

$<0.010$

$<0.010$

$<0.100$

$<0.010$

$<0.010$

$<0.010$

$<1.00<0.010$

28 ..

$<0.010$

$<0.010$

$<0.010$

$<0.010$

$<0.100$

$<0.010$

$<0.010$

$<0.010$

$<1.00$

$<0.010$ 


\section{IILINOIS RIVER SUBBASIN}

\section{FLINT CREEK NEAR WEST SILOAM SPRINGS, OKIABOMA}

IOCArION.--Lat $36^{\circ} 12 \cdot 58^{\prime \prime}$, long $94^{\circ} 36^{\prime} 15^{\prime \prime}$, in NE1/4NE1/4, sec.14, T.20 N., R.25 E., Delaware County, on left bank 180 ft downstream from county bridge, $2.5 \mathrm{mi}$ from Arkansas-oklahoma State line, northwest of Siloam Springs, Oklahoma.

DRATRAE AREA. $--59.8 \mathrm{mi}^{2}$.

AVBRAGE DISCBARGE.--16 years, $51.3 \mathrm{ft}^{3} / \mathrm{s}$.

EXIREus.--June 1979 to current year: Maximum discharge 6,650 $\mathrm{ft}^{3} / \mathrm{s}$ May 3, $1990 ; \mathrm{minimum} \mathrm{daily} 0.40 \mathrm{ft} / \mathrm{s}$ Aug. 7, 1980 .

RERRRs.--Records good except for periods of estimated dally discharges, which are fair. Flow is partially regulated by Lake S1loam Springs, $4.5 \mathrm{mi}$ upstream, and sewage discharge into Flint Creek from city of Gentry.

Monthly and yearly discharge

\begin{tabular}{|c|c|c|c|c|c|}
\hline Month & $\begin{array}{l}\text { Total } \\
\left(\mathrm{ft}^{3} / \mathrm{s}\right)\end{array}$ & $\begin{array}{l}\text { Maximum } \\
\text { daily } \\
\left(\mathrm{ft}^{3} / \mathrm{s}\right)\end{array}$ & $\begin{array}{l}\text { Minimum } \\
\text { daily } \\
\left(\mathrm{ft}^{3} / \mathrm{s}\right)\end{array}$ & $\begin{array}{c}\text { Mean } \\
\left(\mathrm{ft}^{3} / \mathrm{s}\right)\end{array}$ & $\begin{array}{c}\text { Runoff } \\
\text { (acre-feet) }\end{array}$ \\
\hline October & 477 & 22 & 12 & 15.4 & 946 \\
\hline November & 2,615 & 454 & 14 & 87.2 & 5,190 \\
\hline December & 1,557 & 111 & 35 & 50.2 & 3,090 \\
\hline January & 2,590 & 338 & 29 & 83.5 & 5,140 \\
\hline February & 1,387 & 70 & 39 & 49.5 & 2,750 \\
\hline March & 1,809 & 150 & 31 & 58.4 & 3,590 \\
\hline April & 2,267 & 228 & 27 & 75.6 & 4,500 \\
\hline May & 4,844 & 822 & 73 & 156 & 9,610 \\
\hline June & 5,062 & 361 & 75 & 169 & 10,040 \\
\hline July & 1,704 & 90 & 38 & 55.0 & 3,380 \\
\hline August & 745 & 38 & 12 & 24.0 & 1,480 \\
\hline September & 416.1 & 31 & 9.1 & 13.9 & 825 \\
\hline Water year 1995 & $25,473.1$ & 822 & 9.1 & 69.8 & 50,530 \\
\hline
\end{tabular}


07195855 FLINT CRGER MEAR MEST SILOAM SPRINGS, OKLAHOMA--CONTINUED

PERIOD OF RECORD.--June to September 1979, October 1991 to current year.

REMarks.--Specific conductance, pH, water temperature, dissolved oxygen, and alkalinity were determined in the field.

$$
\begin{array}{cc}
\text { DATE } & \text { TIME } \\
\text { JUN } & \\
29 . & 0930 \\
\text { AUG } & \\
03 . & 1030
\end{array}
$$$$
\begin{array}{cc} 
& \\
\text { DATE } & \text { TIME } \\
\text { JUN } & \\
\text { 29. } & 0930 \\
\text { AUG. } & 1030
\end{array}
$$

$$
\text { DATE TIME }
$$$$
\text { JUN } 29.0930
$$$$
\text { AUG } 1030
$$

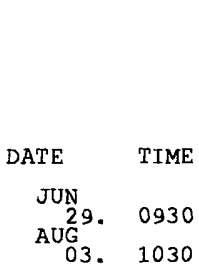
WATER-QUALITY DATA, WATER YEAR OCTOBER 1994 TO SEPTEMBER 1995
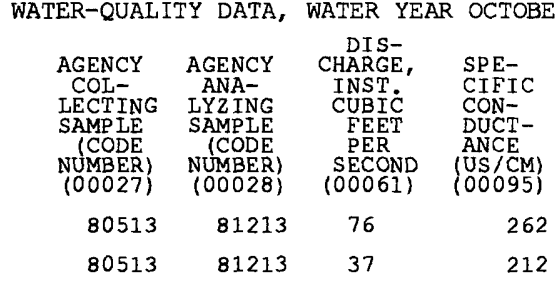

WATER

WHOLE

(STAND-

UNITS)
$(00400)$

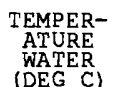

WATER

DEG C)

OXYGEN,

DIS-
SOLVED
(MG /L) (MG/L)

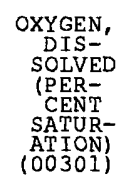

COLI-

$$
7.5
$$

22.0

6.6

25.0

7.0

78
88

70

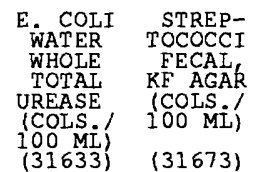

HARD-
NESS
TOTAL
(MG/L
AS
CACO3)
$(00900)$

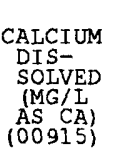

MAGNE-
SIUM,
DIS-
SOLVED
(MG /L
AS MG)
$(00925)$
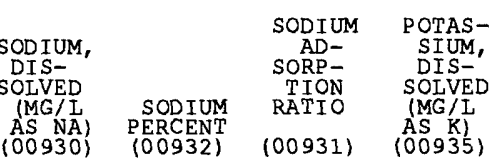

$\begin{array}{ll}70 & 180 \\ 42 & 170\end{array}$

$\begin{array}{rr}110 & 42 \\ 96 & 35\end{array}$

$1.7 \quad 17$

2.19 .3

SOLIDS

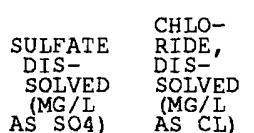

(MG/ I

(00945)

AS CL)
$(00940)$

AT 180
DEG. C

SOLVED

(7030)

NITRO-

NITRO-

$\begin{array}{cc}\text { GEN, } & \text { GEN, } \\ \text { NITRATE } & \text { NITRITE } \\ \text { GEN, } & \text { GEN }\end{array}$

DIS- $\mathrm{NO}_{2+\mathrm{NO}}$

(MG/L

AS N)
$(00613)$

(MG/L
AS N)
$(00618)$

$8.3 \quad 9.1$

162

2.79

0.010

(MG/L
AS N)
$(00630)$

2.80

0.010

0.980

24

17

0.7

0.4

2.8

0.970

PHOS-

NITRO-

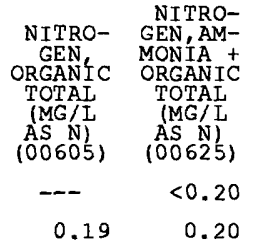

PHOS-
PHORUS
TOTAL
(MG/L
AS P)
$(00665)$

PHOS-

PHORUS

SOLVED

AS P)

0.090

(MG/I

(00671)

0.120

0.020

0.020

0.040

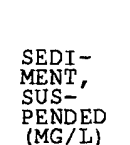

$(80154)$

NITRO- NITRO-

GEN,
NO2+NO3
DIS-

SOLVED SOLVED

(MG/L (MG/L

AS N) $\quad$ AS N)
$(00631) \quad(00608)$

$2.80 \quad 0.010$

0.980

0.010

SEDI- SED.

MENT, SIESP.

CHARGE,

PENDED

(80155) (70331)

9.490

$2.9 \quad 99$ 
LOCATION.--Lat $36^{\circ} 11^{\prime} 11^{\prime \prime}$, long $94^{\circ} 42^{\prime} 24^{\prime \prime}$, in SW1/4NW1/4, sec. 25, T.20 N., R.24 E., Deleware County, Hydrologic Unit 11110103, at U.S. Highway 412 bridge, $6.0 \mathrm{mi}$ southeast of Kansas, and at $\mathrm{mi} 2.2$.'

DRATMAGE AREA.--110 $\mathrm{ml}^{2}$.

PIRTOD OF RECORD. --Water years 1955-61, 1963, 1975-80, July 1991 to current year.

RERARKS.--Specific conductance, $\mathrm{pH}$, water temperature, dissolved oxygen, and alkalinity were determined in the field.

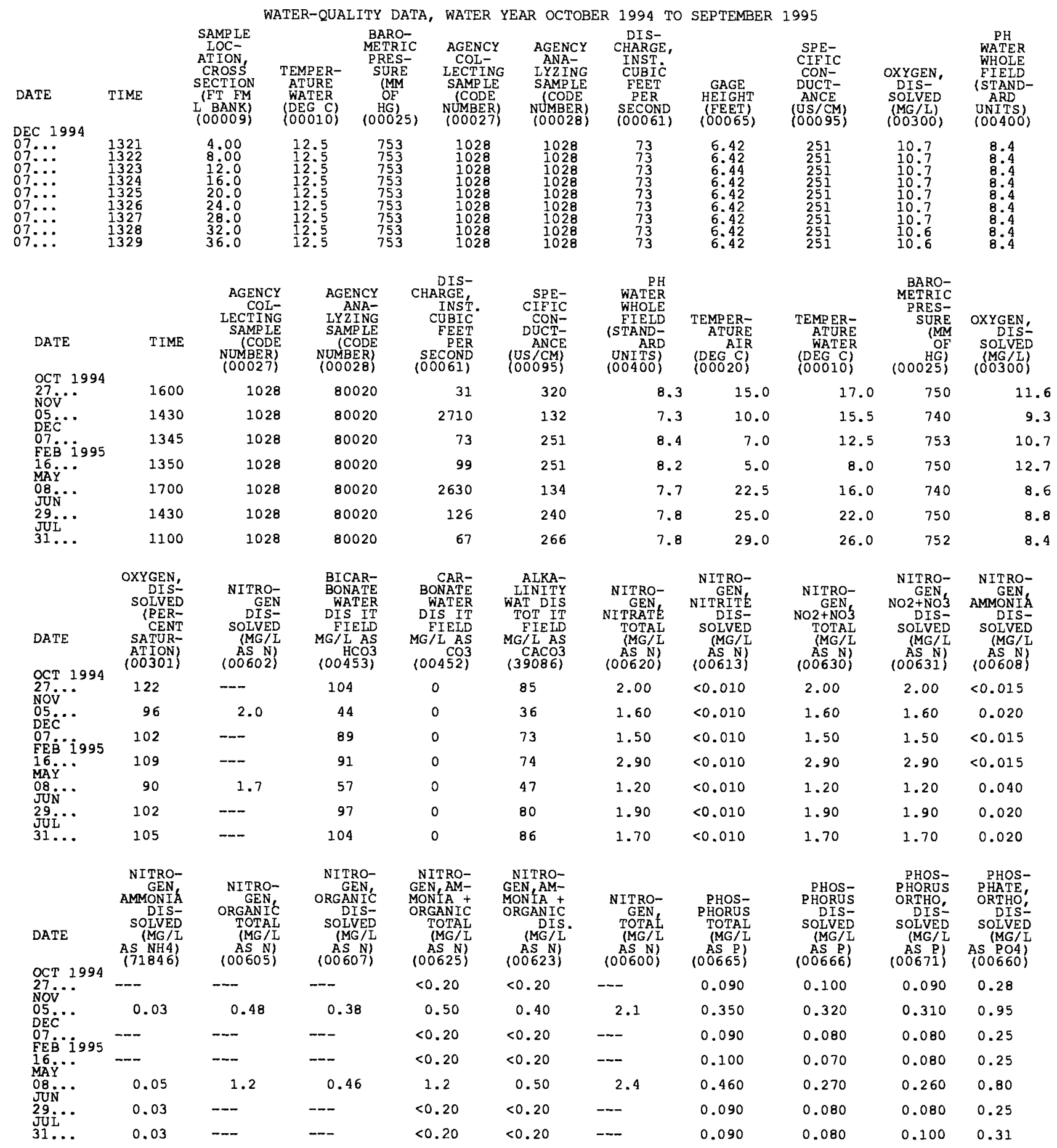




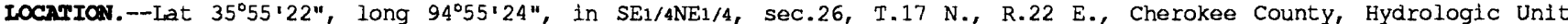
11110103, near center of channel on downstream side of pier of bridge, $0.2 \mathrm{ml}$ downstream from U.S. Highway 62, 2.2 $\mathrm{mi}$ northeast of Tahlequah, $6.5 \mathrm{ml}$ upstream from Baron Fork, and at mile 55.8 .

DRADUER ARBA.--959 $\mathrm{mi}^{2}$.

PERIOD OF RBCORD. - Water years 1960-61, 1975-79, 1989 to current year.

REARKS.--Specific conductance, pH, water temperature, dissolved oxygen, and alkalinity were determined in the field.

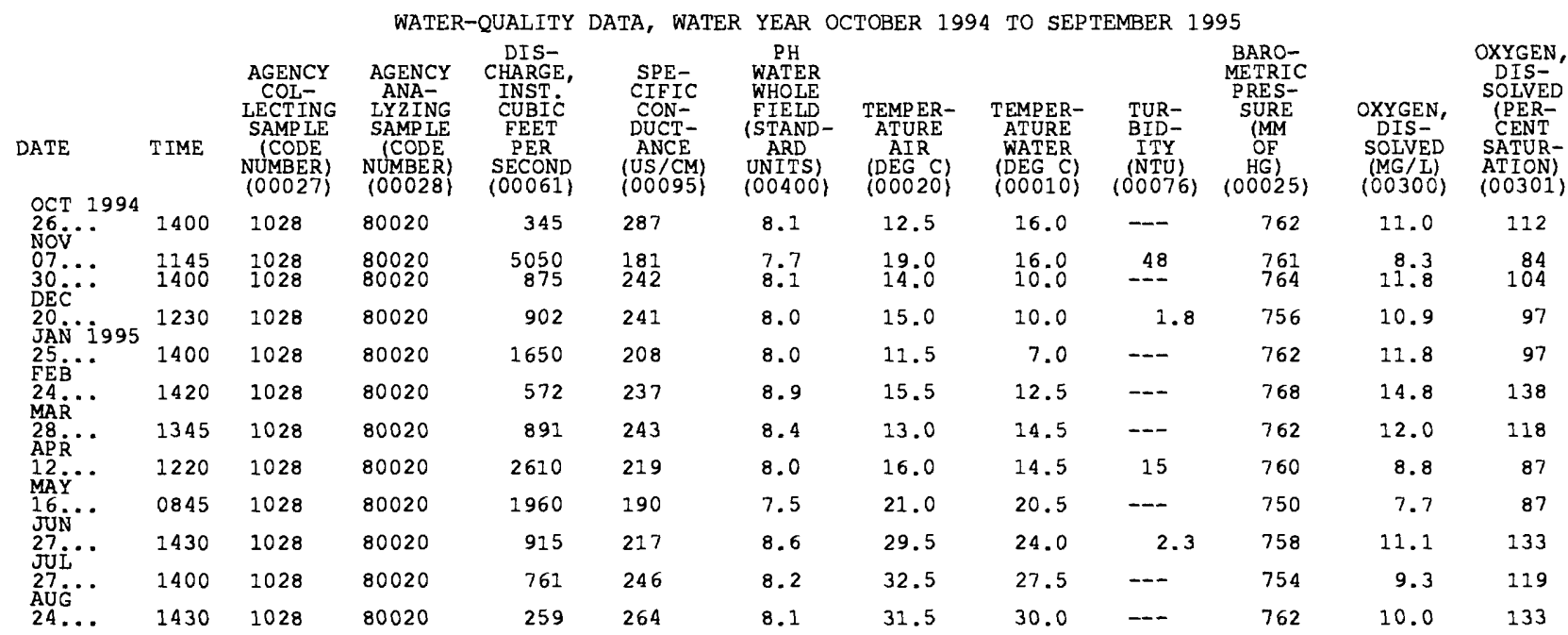

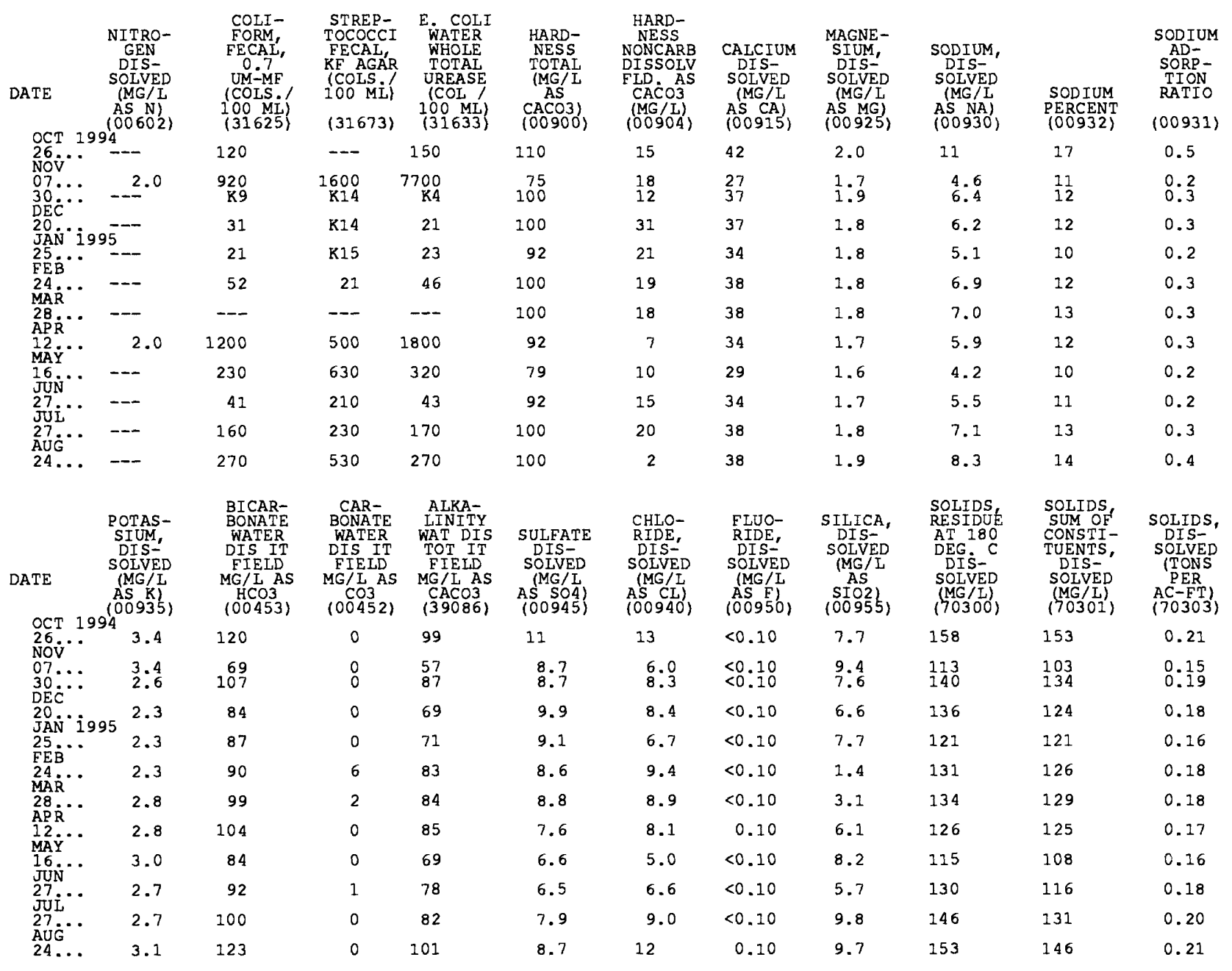


IILINOIS RIVER SUBBASIN

07196500 ILLINOIS RIVER MEAR TABLROOAB, OKLAHOMA--CONTINUED

WATER-QUALITY DATA, WATER YEAR OCTOBER 1994 TO SEPTEMBER 1995

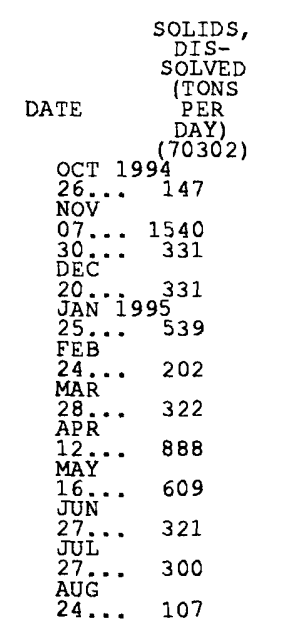

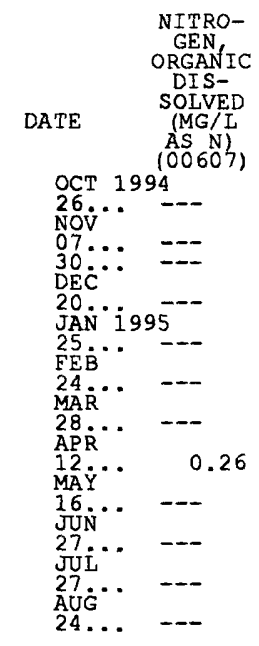

BARIUM,
DIS-
SOLVED
(UG/L
DATE BA
AS BA)
$(01005)$

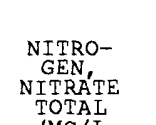

$A S N)$
$(00620)$

0.810

$\frac{1}{2.80}$

2.19

2.50

1.59

1.79

1.69

1.90

1.58

1.30

0.660

NITRO-

MEN AM

ORGANIC

TOTAL

AS N $)^{2}$
$(00625)$

$<0.20$

$<0.50$

$<0.20$

$<0.20$

$<0.20$

0.20

0.40

0.30

0.20

$<0.20$

$<0.20$

BERYL-

LIUM,

DIS-
SOLVED
IUG

AS BE)

10101

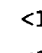

$<1$

$<1$

$<$

$<1$

$<1$

$<1$

$<1$

$-$

AS (D)

20$$
<1 .
$$

$<1.0$
NITRO- NITRO-
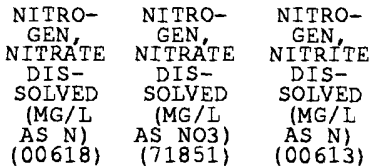

100613)

$<0.010$

$<0.010$

0.010

$<0.010$

0.010

0.010

0.010

$<0.010$

0.020

$<0.010$

$<0.010$

NITRO-

MEN, AM-

MONIA + NITRO-

DIS.

(MG)

AS N)
$(00623) \quad(00600)$

$<0.20 \quad--$

$<0.20 \quad 2.3$

$<0.20 \quad-$.

$<0.20 \quad-$.

$<0.20 \quad \cdots$

$<0.20 \quad 2.0$

$$
0.30 \quad 2.1
$$

$<0.20 \quad 2.2$

$<0.20 \quad 1.8$

$<0.20 \quad \ldots$

$<0.20 \quad \ldots$

BORON, CADMIUM

DIS-
SOLVED SOIS-

(UGG/L

AS B)

-.-

101025

0.080

CHRO-

MIUM,
DIS-
SOLVED

(USG/L

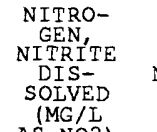

NITRO-
GEN
NO2+NO3
TOTAL
(MG/I
AS N)
$(00630)$

AS NO2

-.. $\quad 0.810$

$-=$

0.03

$--$

0.03

0.03

0.03

$-$.

$0.07 \quad 1.60$

-.-

PHOS-
PHORUS

SOLVED

SOLVED

AS P)
$(00666)$

0.070

0.150

0.060

0.050

0.030

0.060

0.100

0.110

0.060

0.060

0.060

$\begin{array}{rcr}20 & <1.0 & <1 \\ -10 & <1.0 & 1 \\ -- & -- & -- \\ \cdots- & <1.0 & 1 \\ \cdots- & -\cdots & -- \\ -- & <1.0 & 3 \\ -- & -- & -- \\ 20 & <1.0 & <1 \\ -- & --- & --- \\ -- & <1.0 & <1\end{array}$

$\frac{1}{2.80}$

2.20

2.50

1.60

1.80

0.660

PHOS-
PHORUS

ORTHO

DIS-

SOLVED

AS P )
$(00671)$

0.070

0.140

0.070

0.060

0.030

0.060

0.110

0.110

0.050

0.080

0.070

COBALT, COPPER,

DIS-
SOLVED

AS CO)

DIS-

SOLVED

AS CU)
(01040)

(0103)
NITRO-

GEN

DIS-

(MG/L

AS N)
$(00631)$

0.810

$\frac{1}{2.80}$

2.20

2.50

1.60

1.80

1.70

1.90

1.60

1.30

0.660

PHOS-
PHATE,
ORTHO,

DIS-
SOLVED

AS PO4)
$(00660)$

0.21

0.43

0.21

0.21

0.18

0.09

0.18

0.34

0.34

0.15

0.25

0.21

IRON,

DIS-

(UG / L

AS FE)
$(01046)$

NITRO-

AMMONIA

DIS-

SOLVED

AS N)
$(00608)$

$<0.015$

$<0.015$

$<0.015$

$<0.015$

$<0.015$

$<0.015$

$<0.015$

0.040

$<0.015$

0.040

$<0.015$

0.020

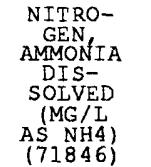

NITRO-

GEN
ORGANIC

(MG N

(00605)

-.. -..

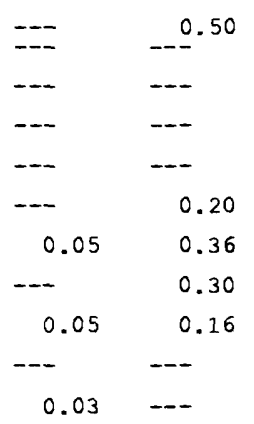

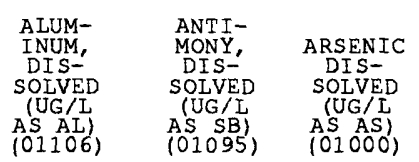

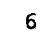

$<1$

$<1$

120

7

$-\cdots$

7

30

--.

10

$--$

$\begin{array}{rrr}<1 & <1 & \\ <1 & 1 & 85 \\ -<1 & 2 & 11 \\ --- & -\cdots & 18 \\ <1 & <1 & 10 \\ -- & -\cdots & 15 \\ <1 & 1 & 37 \\ -- & -\cdots & 76 \\ <1 & 2 & 10 \\ -- & --- & 4 \\ <1 & 1 & <3\end{array}$

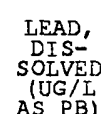

LEAD,
DIS-
SOLVED
(UG/L
(US

AS PB)
$(01049)$

$<1$

$<1$

$<1$

-..

$<1$

-..

$<1$

$--$

$<1$

$--$ $\leq 1 \quad \leq 1$

$<1<1$

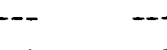

$<1 \quad<1$

$<1 \quad-\cdots$

$\begin{array}{ll}-- & -- \\ <1 & <1\end{array}$

LITHIUM MANGADISSOLVED

$\begin{array}{ll}\text { AS LI) } & \text { AS MN) } \\ (01130) & (01056)\end{array}$

$\begin{array}{cc}-- & 6 \\ <4 & 9 \\ - & 3 \\ <4 & 2 \\ \cdots- & 3 \\ -\cdots & 2 \\ -- & 4 \\ \cdots- & 6 \\ \cdots- & 6 \\ <4 & 2 \\ -- & 4 \\ --- & 6\end{array}$

$<1<1$ 
WATER-QUALITY DATA, WATER YEAR OCTOBER 1994 TO SEPTEMBER 1995
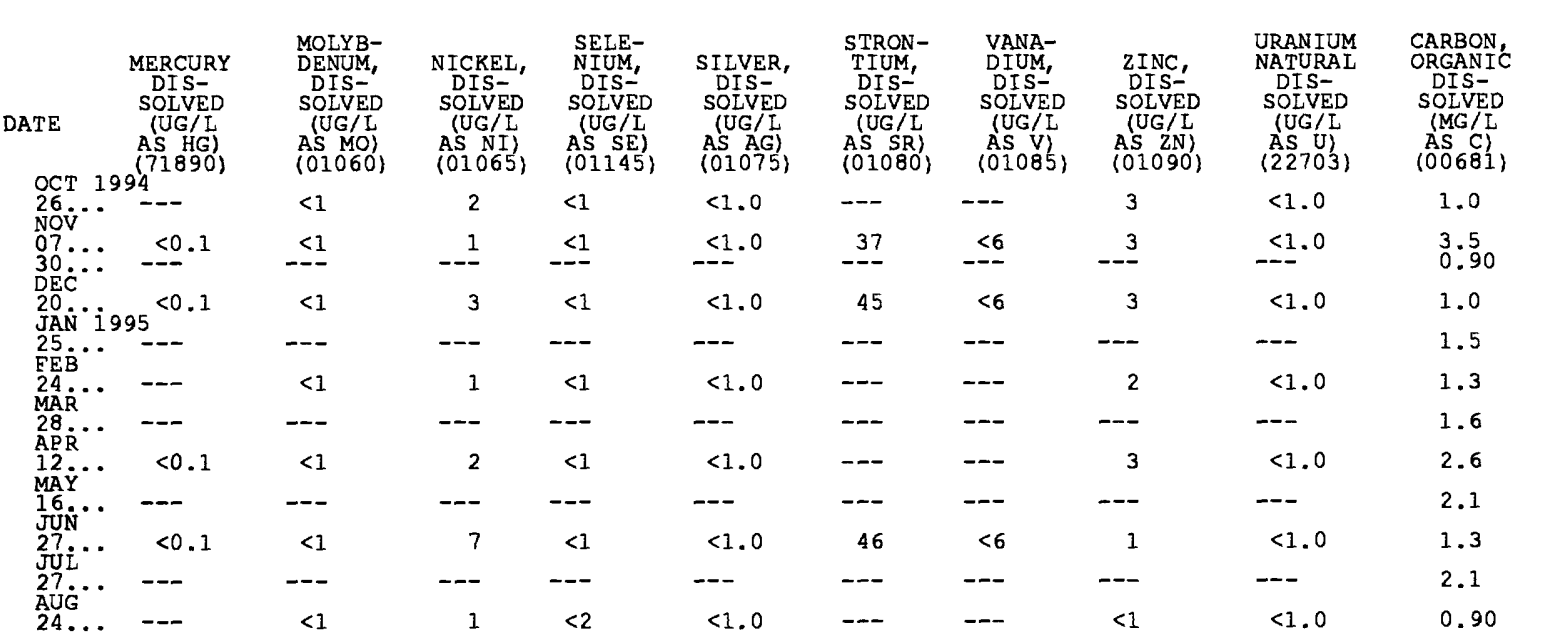

CARBON,

PENDED

(MG/L

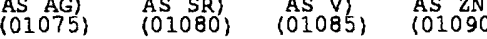

$(22703)$

AS C)
$00689\}$

$<1.0 \quad---\quad--<\quad 3 \quad<1.0$

1.0

0.20

$\begin{array}{rlrl}<1.0 & 37 & <6 & -3\end{array}$

$<1.0$

3.59

0.20

$<1.0 \quad 45 \quad<6$

$<1.0$

0.10

$\begin{array}{rrrr}<1.0 & --- & -- & -- \\ <1.0 & --- & --- & 2\end{array}$

$--$

1.0

0.30

$<1.0$

0.20

$\begin{array}{rrrr}<1.0 & --- & -- & -- \\ --1.0 & --- & --- & 3\end{array}$

$--$

$<1.0$

1.6

0.50

0.90

$--$

$<1.0$

0.60

$<1.0 \quad 46 \quad<6 \quad 1$

$--$

$<1.0 \quad---\quad--><1$

$<1.0$

0.40

1.3

0.20

PESTICIDE ANALYSIS, WATER YEAR OCTOBER 1994 TO SEPTEMBER 1995

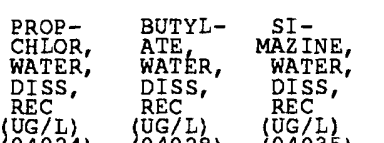

DATE TIME

$\begin{array}{ll}\text { APR } & \\ 12 . & --- \\ M A Y & \\ 16 . & --- \\ \text { JUN } & 27 . \\ \text { JUL } & -- \\ 27 . & ---\end{array}$

DATE TIME

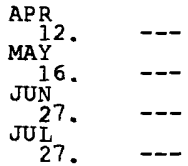

DATE TIME

$\begin{array}{ll}\text { APR } & \\ \text { MY } & \\ \text { MA } & -- \\ \text { JUN } & -- \\ \text { JU. } & \\ \text { JUL } & \\ 27 . & --\end{array}$

$\begin{array}{ll}\text { DATE } & \text { TIME } \\ \text { APR } & \\ 12 . & -- \\ \text { MAY } 16 . & -- \\ \text { JUN } & - \\ 27 . & -- \\ \text { JUL } 27 . & --\end{array}$

DATE TIME

$\begin{array}{ll}\text { APR } & \\ 12 . & -- \\ \text { MAY } & \\ 16 . & -- \\ \text { JUN } & \\ 27 . & -- \\ \text { JUL } \\ 27 . & --\end{array}$

SOLVED

(38933)

$<0.036$

$<0.004$

$<0.00$

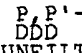

UNFILT

(UG/L)

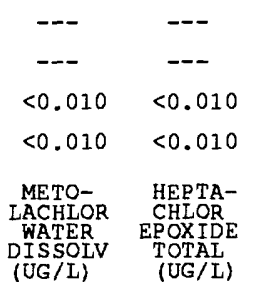$$
\begin{array}{ll}
(\mathrm{UG} / \mathrm{L}) & (\mathrm{UG} / \mathrm{L}) \\
(39415) & (39420)
\end{array}
$$$$
<0.009
$$$$
<0.002
$$$$
<0.002
$$
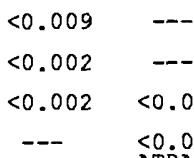

$<0.010$

$<0.010$

METHYL
EARA-
THION,
TOTAL

ATRA-
ZINEE,
WATER,
DISS,

DISS,
REC
(UG/L)
$(39632)$

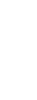

0.017

$<0.010$

$<0.010$

METH-

(UG/L)
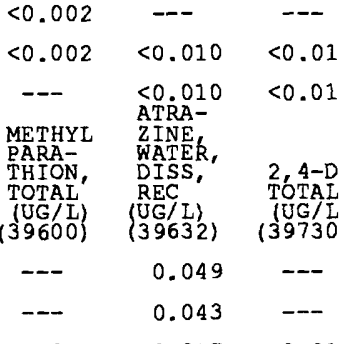

0.049
0.043

$<0.020$

0.017

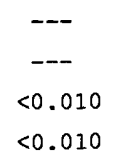

CHLOR- DISUL-

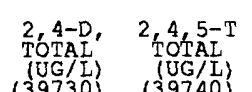
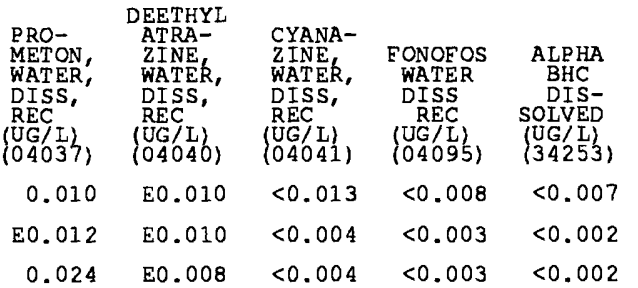

P,P
DDE
DISSOLV
(UG $/$ L

$\left\{\begin{array}{l}\mathrm{UG} / \mathrm{L}) \\ 34653\}\end{array}\right.$

(04041)

$<0.013$

(04095)

$<0.008$

$<0.007$

$<0.004$

$<0.003$

$<0.002$

$<0.010$

$<0.006$

0.024

$<0.004$

$<0.003$

$<0.002$

$<0.006$

$<0.010$

CHLOR-

LINDANE

DIS-

PER-
PHORATE THATE
TOTAL TOTAI

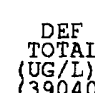

PCNS
UNFILT ALDRIN, LINDANE
RECOVER TOTAL, TOTAL

$\begin{array}{lr}\text { RECOVER } & \text { TOTAL } \\ \left(\begin{array}{l}\text { UG/L) } \\ 39250)\end{array}\right. & (39330)\end{array}$

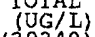

(UG/L)

(UGG/L)

$<0.011$

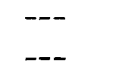

---

---

$<0.004$

$<0.010$

$<0.010$

$<0.004$

$<0.010$

$<0.010$

$---$

0.100

$<0.010$

$<0.100$

DI- ENDO-

$\begin{array}{ll}\text { PAP'- } & \\ \text { DDT } & \text { DI- } \\ \text { UNFILT } & \text { ELDRIN } \\ \text { RECOVER TOTAL } & \text { TOTAL }\end{array}$ (UG/L)
$39370)$

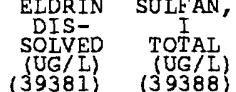

ENDRIN

WATER
NELTRD
REC

ETHION,
TOTAL,
UGG/L

TOX-
APHENE,
TOTAL
(UG/L)
$(39400)$

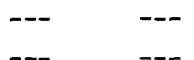

$<0.030$

$<0.001$

$<0.001$

$-$

$<0.010$

$<0.001$

$<0.010$

$--$

$--$

$<0.010$

$<0.010$

$<0.020$

$<1.00$

$<0.010$

CHLOR, PCB

TOTAL

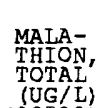

MALA-

THION,
DIS-
SOLVED

(UG/L)
(39532)

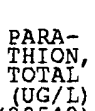

$<0.010$

$<1.00$

$<0.010$

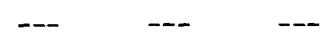

$<0.014$

(39540)

PARA-
THION

THION,
DIS
SOLVED

(UG/L)

DI-
TOINON,
TOTAL

(UG/L)

AZ INI -

AZ INON,

(UG/L)

$<0.022$

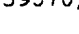

$<0.008$
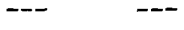

$<0.005$

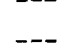

$<0.004$

$<0.010<0.100$

$<0.020$

$<0.005$

$<0.020$

$<0.004$

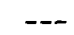

0.017

$<0.010$

ALA-

CHLOR,

$<0.020$

$<0.002$

$<0.010$

ACETO-

TOTAL WATER,

THION

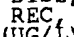

REC

(46342)

FLTRD 2 TOTAL

(UG/L) (UG/L)
$(49260)$
$(82183)$

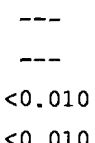

$<0.009$

$<0.009$

$<0.002$

$<0.002$

$<0.010$

$<0.010$

$<0.020$

$<0.002$

$<0.002$

$<0.010$

$<0.010$ 
ILIINOIS RIVER SUBBASIN

07196500 ILLINOIS RIVER NEAR TABLEQDAB, OKLAHONA--CONTINOED

PESTICIDE ANALYSIS, WATER YEAR OCTOBER 1994 TO SEPTEMBER 1995

\begin{tabular}{|c|c|c|c|c|c|c|c|c|c|c|c|}
\hline ATE & TIME & $\begin{array}{l}\text { FONOFOS } \\
\text { (DY- } \\
\text { FONATE) } \\
\text { WATER } \\
\text { WHOLE } \\
\text { TOT.REC } \\
\text { (UG II) } \\
\text { (82614) }\end{array}$ & $\begin{array}{l}\text { METRI- } \\
\text { BUZIN } \\
\text { SENCOR } \\
\text { WATER } \\
\text { DISSOLV } \\
(\mathrm{UG} / \mathrm{L}) \\
(82630)\end{array}$ & $\begin{array}{l}2,6-D I- \\
\text { ETHYI } \\
\text { ANIIINE } \\
\text { WAT EIT } \\
0.7 \text { U } \\
\text { GF' REC } \\
(\text { UG II) } \\
(82660)\end{array}$ & $\begin{array}{l}\text { TRI- } \\
\text { FIUR- } \\
\text { ALIN } \\
\text { WAT FLT } \\
0.7 \text { U } \\
\text { GF' REC } \\
\text { (UG/L) } \\
(82661)\end{array}$ & $\begin{array}{c}\text { ETHAL- } \\
\text { FLUR- } \\
\text { ALIN } \\
\text { WAT FLT } \\
0.7 \text { U } \\
\text { GF' REC } \\
\text { (UG/L) } \\
(82663)\end{array}$ & $\begin{array}{l}\text { PHORATE } \\
\text { WATER } \\
\text { FLTRD } \\
0.7 \mathrm{U} \\
\text { GF. REC } \\
(\mathrm{UG} / \mathrm{RE}) \\
(82664)\end{array}$ & $\begin{array}{l}\text { TER- } \\
\text { BACIL } \\
\text { WATER } \\
\text { ELTRD } \\
0.7 \mathrm{U} \\
\text { GF' REC } \\
(\mathrm{UG} / \mathrm{L}) \\
(82665)\end{array}$ & $\begin{array}{c}\text { LIN- } \\
\text { URON } \\
\text { WATER } \\
\text { FITRD } \\
0.7 \mathrm{U} \\
\text { GE } \\
(\mathrm{UGE} / \mathrm{REC} \\
(82666)\end{array}$ & $\begin{array}{c}\text { METHYL } \\
\text { PARA- } \\
\text { THION } \\
\text { WAT FIT } \\
0.7 \text { U } \\
\text { GF' REC } \\
\text { (UG/I) } \\
(82667)\end{array}$ & $\begin{array}{c}\text { EPTC } \\
\text { WATER } \\
\text { FLTRD } \\
0.7 \mathrm{U} \\
\text { GF } 7 \text { REC } \\
(\mathrm{UG} / \mathrm{L}) \\
(82668)\end{array}$ \\
\hline & -.. & -- & $<0.012$ & $<0.006$ & $<0.012$ & $<0.013$ & $<0.011$ & $<0.030$ & $<0.039$ & $<0.035$ & $<0.005$ \\
\hline & -- & -- & $<0.004$ & $<0.003$ & $<0.002$ & $<0.004$ & $<0.002$ & $<0.007$ & $<0.002$ & $<0.006$ & $<0.002$ \\
\hline & -- & $<0.020$ & $<0.004$ & $<0.003$ & $<0.002$ & $<0.004$ & $<0.002$ & $<0.007$ & $<0.002$ & $<0.006$ & $<0.002$ \\
\hline $\mathrm{AT}$ & TIME & $\begin{array}{l}<0.010 \\
\text { PEB } \\
\text { ULATE } \\
\text { WATER } \\
\text { FILTRD } \\
0.7 \mathrm{U} \\
\text { GE; REC } \\
\text { (UG/L) } \\
(82669)\end{array}$ & $\begin{array}{l}\text { TEBU- } \\
\text { THIURON } \\
\text { WATER } \\
\text { FLTRD } \\
0.7 \mathrm{U} \\
\text { GE' REC } \\
(\mathrm{UG} / \mathrm{R}) \\
(82670)\end{array}$ & $\begin{array}{c}\text { MOL- } \\
\text { INATE } \\
\text { WATER } \\
\text { FLTRD } \\
0.7 \mathrm{U} \\
\text { GF; REC } \\
(\mathrm{UG} / \mathrm{R}) \\
(82671)\end{array}$ & $\begin{array}{l}\text { ETHO- } \\
\text { PROP } \\
\text { WATER } \\
\text { FLTRD } \\
0.7 \mathrm{U} \\
\text { GF } \\
\text { (UG/I) } \\
(82672)\end{array}$ & $\begin{array}{c}\text { BEN- } \\
\text { BEN- } \\
\text { FLUR } \\
\text { ALIN } \\
\text { WAT FLD } \\
0.7 \mathrm{U} \\
\text { GE' REC } \\
(\mathrm{UG} / \mathrm{R}) \\
(82673)\end{array}$ & $\begin{array}{l}\text { CARBO- } \\
\text { FURAN } \\
\text { WATER } \\
\text { FLTRD } \\
0.7 \mathrm{U} \\
\text { GF' REC } \\
(\mathrm{UG} / \mathrm{L}) \\
(82674)\end{array}$ & $\begin{array}{c}\text { PER- } \\
\text { TER- } \\
\text { BUFOS } \\
\text { WATER } \\
\text { FLTRD } \\
0.7 \mathrm{U} \\
\text { GEF REC } \\
(\mathrm{UG} / \mathrm{R}) \\
(82675)\end{array}$ & $\begin{array}{l}\text { PRON- } \\
\text { AMIDE } \\
\text { WMTER } \\
\text { FLTRD } \\
0.7 \mathrm{U} \\
\text { GE } 7 \text { REC } \\
(\mathrm{UGG} / \mathrm{L}) \\
(82676)\end{array}$ & $\begin{array}{l}\text {--- } \\
\text { DISUL- } \\
\text { FOTON } \\
\text { WATER } \\
\text { FLTRD } \\
0.7 \mathrm{U} \\
\text { GF'REC } \\
(\mathrm{UG} / \mathrm{L}) \\
(82677)\end{array}$ & $\begin{array}{l}\text { TRIAL- } \\
\text { LATE } \\
\text { WATER } \\
\text { FLTRD } \\
0.7 \text { U } \\
\text { GF; REC } \\
\text { (UG/L) } \\
\{82678)\end{array}$ \\
\hline & $\cdots$ & $<0.009$ & E0.015 & $<0.007$ & $<0.012$ & $<0.013$ & $<0.013$ & $<0.012$ & $<0.009$ & $<0.060$ & $<0.008$ \\
\hline & -- & $<0.004$ & 0.023 & $<0.004$ & $<0.003$ & $<0.002$ & $<0.003$ & $<0.013$ & $<0.003$ & $<0.017$ & $<0.001$ \\
\hline 21 & -- & $<0$. & 0.011 & 04 & 03 & 0.002 & 0.003 & 0.013 & $<0.003$ & 0.017 & 01 \\
\hline
\end{tabular}

$\begin{array}{cc}\text { DATE } & \text { TIME } \\ \text { APR } & \\ 12 . & - \\ \text { MAY } & - \\ \text { I6. } & -- \\ \text { JUN } & - \\ 27 . & -\end{array}$

\begin{tabular}{|c|c|c|c|}
\hline $\begin{array}{c}\text { PRO- } \\
\text { PANIL } \\
\text { WATER } \\
\text { FLTRD } \\
0.7 \text { U } \\
\text { GE; REC } \\
\left(\begin{array}{l}\text { UG } / L) ~ \\
(82679)\end{array}\right.\end{array}$ & $\begin{array}{c}\text { CAR- } \\
\text { BARYL } \\
\text { WATER } \\
\text { FLTRD } \\
0.7 \text { U } \\
\text { GE; REC } \\
\langle\text { UG /L } \\
\langle 82680\rangle\end{array}$ & $\begin{array}{c}\text { THIO- } \\
\text { BENCARB } \\
\text { WATER } \\
\text { FLTRD } \\
0.7 \text { U } \\
\text { GF', REC } \\
\left(\begin{array}{l}\text { UG } / L) \\
(82681)\end{array}\right.\end{array}$ & $\begin{array}{c}\text { DCPA } \\
\text { WATER } \\
\text { FLTRD } \\
0.7 \mathrm{U} \\
\text { GE; REC } \\
(U G / I) \\
(82682)\end{array}$ \\
\hline$<0.016$ & $<0.046$ & $<0.008$ & $<0.004$ \\
\hline$<0$. & E0.044 & $<0.002$ & $<0.002$ \\
\hline & $<0.003$ & $<0.002$ & 002 \\
\hline
\end{tabular}

$\begin{array}{cc}\text { PENDI- } & \text { NAPROP- } \\ \text { METH- } & \text { AMIDE } \\ \text { ALIN } & \text { WATER } \\ \text { WAT FLT } & \text { FLTRD } \\ \text { O.7 U } & 0.7 \text { U } \\ \text { GE; REC } & \text { GF; REC } \\ (U G / L) & (U G / L) \\ (82683) & (82684) \\ (826) & \\ <0.018 & <0.010 \\ <0.004 & <0.003 \\ <0.004 & <0.003\end{array}$

\begin{tabular}{|c|}
\hline $\begin{array}{c}\text { PRO- } \\
\text { PARGIT } \\
\text { WATER } \\
\text { FLTRD } \\
0.7 \text { U } \\
\text { GE' RE. } \\
\text { (UGG/L) } \\
82685\end{array}$ \\
\hline$<0$. \\
\hline \\
\hline \\
\hline
\end{tabular}

METHYL
AZIN-
PHOS
WAT FLT
$0.7 \mathrm{U}$
GF' REC
$\langle\mathrm{UG} / \mathrm{L}$ )
$\langle 8268$ )
$<0.038$
$<0.001$
$<0.001$

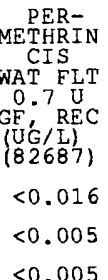




\section{ITLINOIS RIVER SUBBASIN}

\section{BARON FORK AT DUTCE MILLS, ARRANSAS}

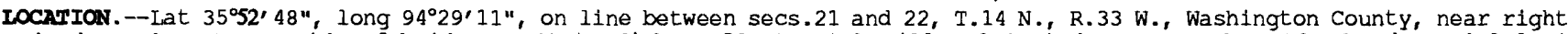
bank on downstream side of bridge on State Highway 59 at Dutch Mills, 2.2 mi downstream from Fly Creek, and 2.9 mi upstream from Arkansas-Oklahoma state line.

DRATMEE AREA. $--40.6 \mathrm{mi}^{2}$.

AVRRACE DISCHAReE. -37 years, $45.0 \mathrm{ft}^{3} / \mathrm{s}$.

EXIREArs.--April 1958 to current year: Maximum discharge 20,900 ft ${ }^{3} / \mathrm{s}$ Nov. 18, 1985; no flow at times.

REMRRKS.--Records good. Satellite telemeter at station.

Monthly and yearly discharge

\begin{tabular}{|c|c|c|c|c|c|}
\hline Month & $\begin{array}{l}\text { Total } \\
\left(\mathrm{ft}^{3} / \mathrm{s}\right)\end{array}$ & $\begin{array}{l}\text { Maximum } \\
\mathrm{dail}^{2} \mathrm{y} \\
\left(\mathrm{ft}^{3} / \mathrm{s}\right)\end{array}$ & $\begin{array}{l}\text { Minimum } \\
\mathrm{dail} \mathrm{y} \\
\left(\mathrm{ft}^{3} / \mathrm{s}\right)\end{array}$ & $\begin{array}{c}\text { Mean } \\
\left(\mathrm{ft}^{3} / \mathrm{s}\right)\end{array}$ & $\begin{array}{l}\text { Runoff } \\
\text { (acre- } \\
\text { feet) }\end{array}$ \\
\hline October & 63.97 & 6.0 & 0.30 & 2.06 & 127 \\
\hline November & $2,422.8$ & 454 & 5.0 & 80.8 & 4,810 \\
\hline December & 1,782 & 399 & 15 & 57.5 & 3,530 \\
\hline January & 4,753 & 1,590 & 16 & 153 & 9,430 \\
\hline February & 1,075 & 68 & 21 & 38.4 & 2,130 \\
\hline March & 1,512 & 356 & 19 & 48.8 & 3,000 \\
\hline April & 2,669 & 941 & 12 & 89.0 & 5,290 \\
\hline May & 4,966 & 1,800 & 21 & 160 & 9,850 \\
\hline June & 2,824 & 617 & 15 & 94.1 & 5,600 \\
\hline July & 539.3 & 114 & 4.4 & 17.4 & 1,070 \\
\hline August & 83.01 & 7.1 & .67 & 2.68 & 165 \\
\hline September & 259.83 & 21 & .50 & 8.66 & 515 \\
\hline Water year 1995 & $22,949.91$ & 1,800 & .30 & 62.9 & 45,520 \\
\hline
\end{tabular}


PERIOD OF RECORD.--October 1960 to September 1961, October 1968 to current year.

REMaRKS.--Specific conductance, pH, water temperature, dissolved oxygen, and alkalinity were determined in the field. WATER-QUALITY DATA, WATER YEAR OCTOBER 1994 TO SEPTEMBER 1995

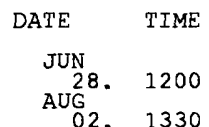

AUG -1200

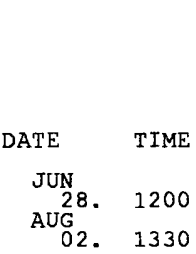

DATE TIME

JUN 28.1200

A2. 1330

$\begin{array}{ll}\text { DATE } & \text { TIME } \\ \text { JUN } & \\ \text { 28. } & 1200 \\ \text { AUG } & 1330\end{array}$

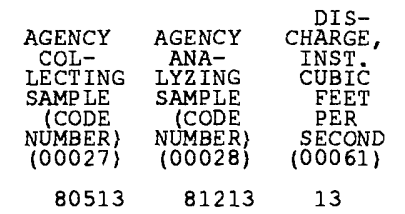

$80513 \quad 81213$

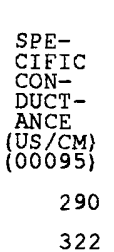

PH
WATER
WHOLE
EIELD
(STAND-
ARD
UNITS

UNTTS
$(00400\}$

7.1

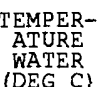

WATER

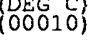$$
22.5
$$

25.0

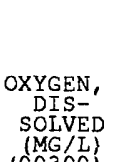
$(00300)$

\section{7}

5.2
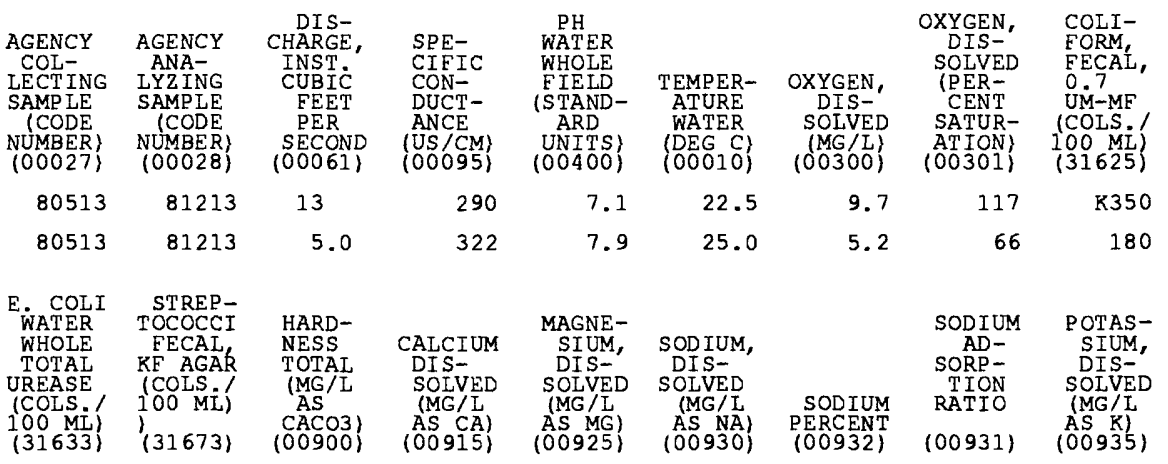

2.9

4.5

3.1

4.9

$150 \quad 56$

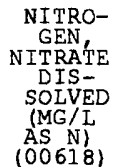
(00618)

2.38

1.28

6.4
NITROGEN,
NITRITE DISSOLVED (MG/L AS N)

0.020

0.020

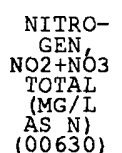
GEN $\mathrm{O} 2+\mathrm{NO}_{3}$ AS N)

2.40

1.30

PHOS- PHORUS

NITRO- NITRO$\begin{array}{ccc}\text { GEN } & \text { MONIA + } \\ \text { ORGANIC } & \text { PHOS- }\end{array}$ $\begin{array}{ll}\text { TOTAL } & \text { TOTAL } \\ \text { (MG/L } & \text { (MG/L }\end{array}$ (00605) AS N $)$
$(00625)$

$$
0.21
$$

0.22

0.26
TOTAL

(MG $/$ L
AS P)
$(00665)$

0.020

DTS

DIS-

SOLVED SOLVED

AS P) AS P)

0.020

0.060

0.040

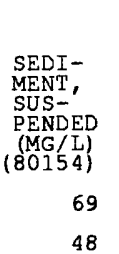

0.040

0.050 $\begin{array}{ll} & \\ \text { AD I } & \text { POTAS- } \\ \text { SIUM }\end{array}$

SORP -

TATIO

(00931)

0.2

0.23 .0 NITRO- NITRONO2+NO3 AMMONIA SOLVED SOLVED (MG/L (MG/L ASN) ASN)
$(00631) \quad(00608)$

$2.40 \quad 0.010$

1.30

0.050

SEDI- $\quad$ SED.

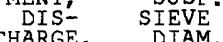

CHARGE, DIAM.

SUS- क FINER

$\begin{array}{ll}(\mathrm{T} / \mathrm{DAY}) & 062 \mathrm{MM} \\ (80155) & (70331)\end{array}$

2.4

85

0.65

89 


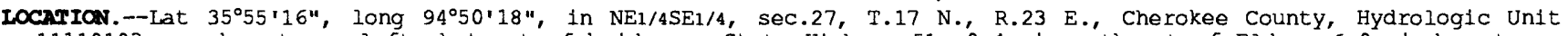
11110103 , on downstream left abutment of bridge on state Highway $51,0.4$ mi southeast of Eldon, 6.0 mi downstream from Tyner Creek, and at mile 8.8.

DRAINAGE ARRA. $--307 \mathrm{mi}^{2}$.

PERIOD OF RECORD. $-1948,1958-60$, 1991 to current year.

REMaRKs.--Specific conductance, $\mathrm{pH}$, water temperature, and dissolved oxygen were determined in the field. WATER-QUALITY DATA, WATER YEAR OCTOBER 1994 TO SEPTEMBER 1995

DATE TIME

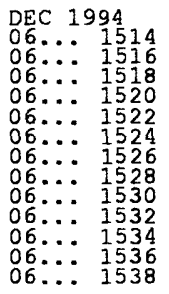

5.00
10.0
15.0
20.0
25.0
30.0
35.0
40.0
45.0
50.0
55.0
60.0
65.0

SAMPLE
IOC-
ATION,
CROSS
SECTION
(FT EM
L BANK)
\{00009)
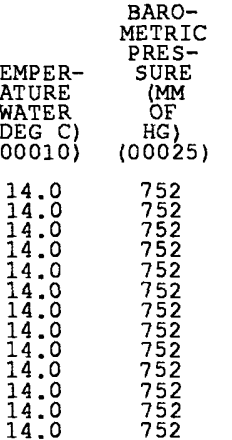
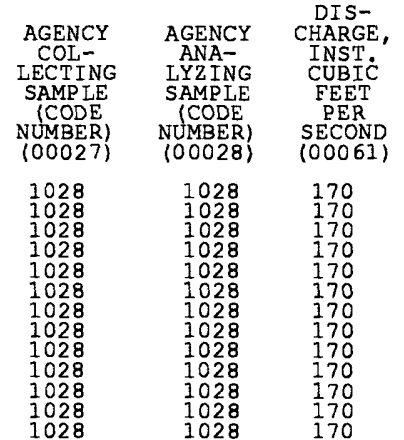
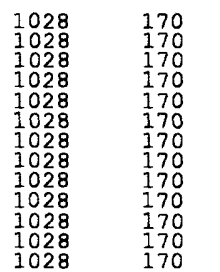

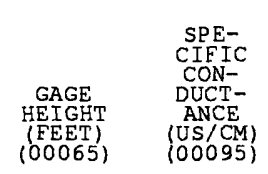

4.76
4.76
4.76
4.76
4.76
4.76
4.76
4.76
4.76
4.76
4.76
4.76
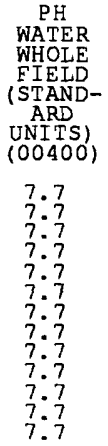

OXYGEN, DIS-
SOLVED (PERCENT

ATION)

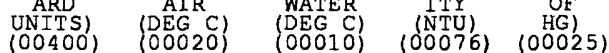

$\begin{array}{rrrcrrr}7.7 & 0.5 & 15.0 & -- & 759 & 8.9 & 89 \\ 7.7 & 19.0 & 14.0 & 0.40 & 752 & 10.7 & 105 \\ 8.0 & 6.0 & 8.5 & -- & 754 & 12.8 & 111 \\ 7.5 & 21.5 & 15.5 & 310 & 745 & 8.5 & 87 \\ 7.9 & 29.5 & 20.0 & -- & 754 & 8.7 & 97 \\ 7.6 & 19.5 & 21.0 & 1.3 & 757 & 7.1 & 80 \\ 7.6 & 30.0 & 24.0 & -- & 754 & 7.7 & 93\end{array}$

* laboratory value instead of field value
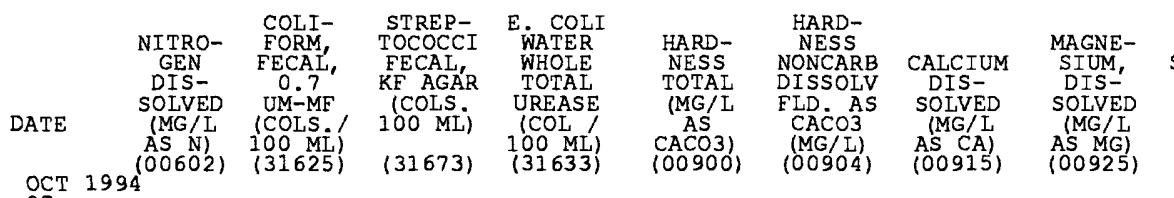

SODIUM,

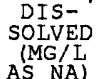

SODIUM

AS MG) AS NA) PERCENT

(00900) (00904) (0091)
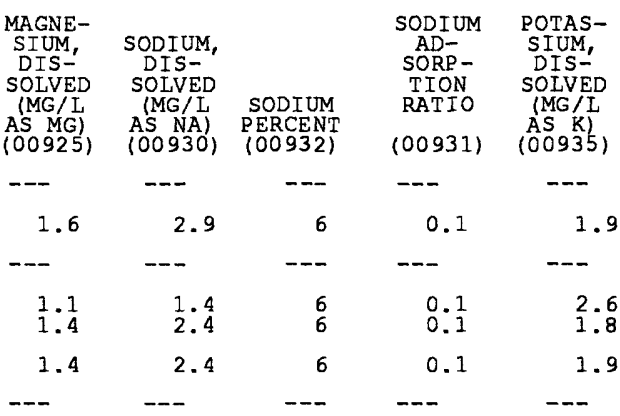

SOLIDS, SOITS
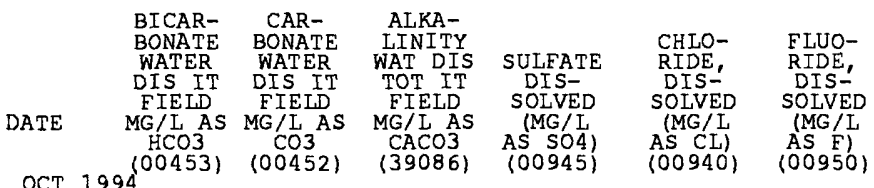

SILICA,

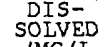

(MG / L

AS

RESIDUE

SOLIDS,

AT 180 CONSTT- SOLIDS,

DEG.C TUENTS, SOLVED

SOLVED

(MG/L)

DIS-
SOLVED

SOLVED PER

(MG/L) AC-ET)

SOLIDS, NITRO-

DIS- GEN,
SOLVED NITRATE

ITONS TOTAL

DAY) AS N)

(70302) (00620)

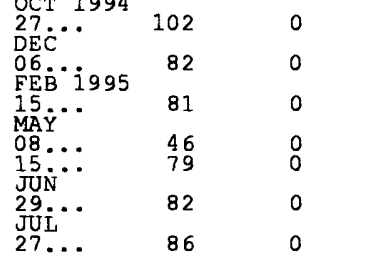

--
6.5
--
3.6
4.0
--
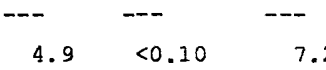

$\frac{1}{3}$

3.

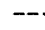

$129 \quad 0.16$

0.450

116

$---$

70
99

59
95

95$$
--
$$$$
0.09
$$$$
6.60
$$$$
1.60
$$$$
\begin{array}{rl}
3360 & 0.430 \\
167 & 1.50
\end{array}
$$$$
0.14
$$

69.4

1.50
-.- -.- -.-

0.990
1.10 
ILIINOIS RIVER SUBBASIN

07197000 BARON FORK AT ELDON, OKLABOMA--CONTINUED

WATER-QUALITY DATA, WATER YEAR OCTOBER 1994 TO SEPTEMBER 1995

\begin{tabular}{|c|c|c|c|c|c|c|c|c|c|c|c|c|}
\hline OCT 1994 & $\begin{array}{c}\text { NITRO- } \\
\text { GEN, } \\
\text { NITRITE } \\
\text { DIS- } \\
\text { SOLVED } \\
\text { (MG/L } \\
\text { AS N } \\
(00613) \\
4(006)\end{array}$ & $\begin{array}{c}\text { NITRO- } \\
\text { GEN } \\
\text { NO2+NO3 } \\
\text { TOTAL } \\
\text { (MG / } \\
\text { AS N } \\
(00630)\end{array}$ & $\begin{array}{c}\text { NITRO- } \\
\text { GEN, } \\
\text { NO2+NO3 } \\
\text { DIS- } \\
\text { SOLVED } \\
\text { (MG/L } \\
\text { AS N) } \\
(00631)\end{array}$ & $\begin{array}{c}\text { NITRO- } \\
\text { GEN, } \\
\text { AMMONIA } \\
\text { DIS- } \\
\text { SOLVED } \\
\text { \{MG/L } \\
\text { AS N } \\
(00608)\end{array}$ & $\begin{array}{c}\text { NITRO- } \\
\text { GEN, } \\
\text { AMMONIA } \\
\text { DIS- } \\
\text { SOLVED } \\
\text { (MG/I } \\
\text { AS NH4) } \\
(71846)\end{array}$ & $\begin{array}{c}\text { NITRO- } \\
\text { GEN } \\
\text { ORGAIC } \\
\text { TOTAI } \\
\text { TMG/I } \\
\text { AS N) } \\
(00605)\end{array}$ & $\begin{array}{c}\text { NITRO- } \\
\text { GEN } \\
\text { ORGANIC } \\
\text { DIS- } \\
\text { SOIVED } \\
\text { (MG/L } \\
\text { AS N) } \\
(00607)\end{array}$ & $\begin{array}{l}\text { NITRO- } \\
\text { GEN,AM- } \\
\text { MONIA + } \\
\text { ORGANIC } \\
\text { TOTAL } \\
\text { (MG/L } \\
\text { ASN } \\
(00625)\end{array}$ & $\begin{array}{l}\text { NITRO- } \\
\text { GEN,AM- } \\
\text { MONIA + } \\
\text { ORGANIC } \\
\text { DIS } \\
\text { (MG/I } \\
\text { AS N } \\
(00623)\end{array}$ & $\begin{array}{c}\text { NITRO- } \\
\text { GEN, } \\
\text { TOTAL } \\
\text { (MG/I } \\
\text { AS N } \\
(00600)\end{array}$ & $\begin{array}{c}\text { PHOS- } \\
\text { PHORUS } \\
\text { TOTAL } \\
\text { (MG/I } \\
\text { AS P) } \\
(00665)\end{array}$ & $\begin{array}{l}\text { PHOS- } \\
\text { PHORUS } \\
\text { DIS- } \\
\text { SOLVED } \\
\text { (MG/L } \\
\text { AS P) } \\
(00666)\end{array}$ \\
\hline DEC & $<0.010$ & 0.450 & 0.450 & $<0.015$ & --- & -- & -- & $<0.20$ & $<0.20$ & --- & 0.010 & 0.020 \\
\hline O6... & $5^{<0.010}$ & 6.60 & 6.60 & $<0.015$ & --- & -- & -- & $<0.20$ & $<0.20$ & --- & 0.030 & 0.030 \\
\hline MAY & $<0.010$ & 1.60 & 1.60 & $<0.015$ & --- & -- & --- & $<0.20$ & $<0.20$ & --- & $<0.010$ & 0.020 \\
\hline $\begin{array}{l}08 \ldots \\
15 \ldots \\
\text { JUN }\end{array}$ & $\begin{array}{l}<0.010 \\
<0.010\end{array}$ & $\begin{array}{c}0.430 \\
1.50\end{array}$ & $\begin{array}{l}0.430 \\
1.50\end{array}$ & $\begin{array}{r}0.040 \\
<0.015\end{array}$ & 0.05 & 2.5 & 0.46 & $\begin{array}{r}2.5 \\
<0.20\end{array}$ & $\begin{array}{l}0.50 \\
<0.20\end{array}$ & 2.9 & $\begin{array}{l}0.960 \\
0.030\end{array}$ & $\begin{array}{l}0.140 \\
0.030\end{array}$ \\
\hline JUL & $<0.010$ & 0.990 & 0.990 & 0.040 & 0.05 & --- & --- & $<0.20$ & $<0.20$ & --- & 0.030 & 0.020 \\
\hline $27 \ldots$ & $<0.010$ & 1.10 & 1.10 & $<0.015$ & --- & --- & --- & $<0.20$ & $<0.20$ & --- & 0.030 & 0.030 \\
\hline
\end{tabular}

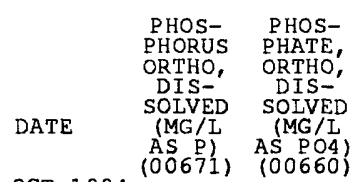

ARSENIC BARIUM, BERYL-

DIS- BARIUM,

SOLVED

BORON,
DIS-
SOLVED
(UG/L
AS B)
(OIO20)

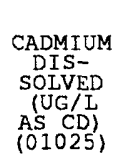

CHRO-

MIUM, COBALT, COPPER, DIS-
SOLVED
(UG/L
(US $/ \mathrm{CR}$

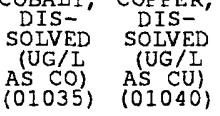

IRON,
DIS-
SOLVED
(USG/L
AS FE)
$(01046)$

LEAD,

SOLVED

$A S$ PB)

$\begin{array}{lll}\text { OCT } 1994 & 0.020 & 0.06\end{array}$

(UG/L (UG/L
AS AS) AS BA)

AS BE)
(01010)

AS CR)
$(01030)$

(1)

FEB ${ }_{\text {O }} 0.095$

$15 \ldots 0.020$

$\begin{array}{ll}\text { MAY } & 0.130 \\ 08 \ldots & 0.130\end{array}$

$\begin{array}{ll}08 \ldots & 0.130 \\ 15 \ldots & 0.030\end{array}$

0.06

$<1$

$<0.5$

$<1.0 \quad<5$

$<3$

$<10$

$<3$

10

$\begin{array}{ll}\text { JUN } & 0.020 \\ \text { JUL } & 0.030\end{array}$

0.06

$-$

$---$

$---\quad--$

$<0.5 \quad 10$

$<1.0 \quad<5$

$<3$

$<10$

430

$0.06<1$

$<0.5$

20

$<1.0<5$

$<3$

$<10$

10

$<10$

27... $0.030 \quad 0.09$

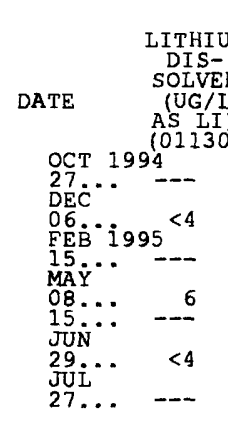

DATE

MAY 1995
$15 .$.

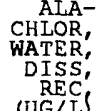

(UG/I

$\{46342\}$

$<0.009$

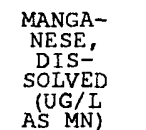

$\begin{array}{cc} & \text { MOLYB- } \\ \text { MERCURY } & \text { DENUM, } \\ \text { DIS- } & \text { DIS- } \\ \text { SOLVED } & \text { SOLVED } \\ \text { (UG/L } & \text { (UG/L } \\ \text { AS HG) } & \text { AS MO) } \\ (71890) & (01060)\end{array}$
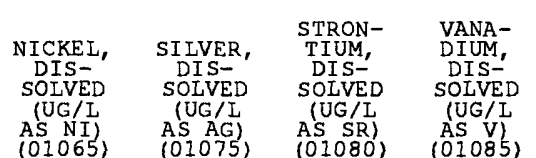

(01080

$<10$

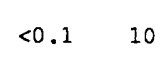

-- - --

$<0.1<10$

$--$

$<10$

$<0.1<10$

8

$<10$
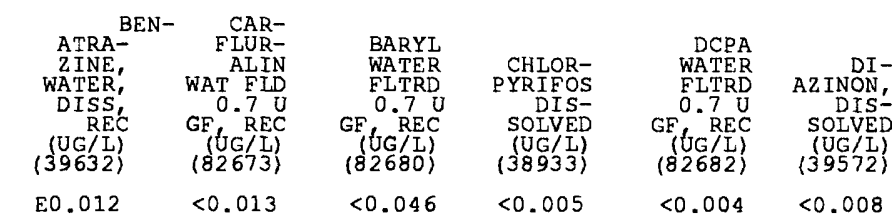

$\begin{array}{crr} & \text { ALPHA } & \\ \text { BHC } & \text { LINDANE } \\ \text { DATE } & \text { DIS- } & \text { DIS- } \\ & \text { SOLVED } & \text { SOLVED } \\ & (U G / L) & (U G / L) \\ & (34253) & (39341)\end{array}$

MAY 1995

(34253)

(39341)

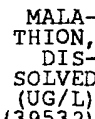

METO-

WACHLOR

DISSOLV

$($ UG $/ L)$
$(39415)$

$<0.009$

$<0.014$

\section{TER-}

TERBUTH

MAZINE,

DATE

DISS,

MAY 1995

$(U G / L)$
$(04035)$

WATER

GF 0.7 REC

$(U G / L)$
$(82665)$

$<0.030$
SURROGT
WAT FLT

GF REC

PERCENT
(91064)

34.7

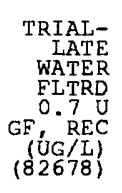

$<0.008$

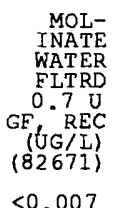

PARA-

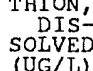

(UG/L)

$<0.022$

DEETHYL

ATNE,
WATER,'
DISS,

(UG/L)

(04040)

E0.008

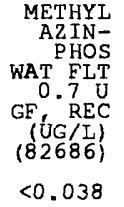

PENDT-

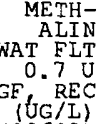

$(\mathrm{UG} / \mathrm{L})$
$(82683)$

$<0.018$

(UG/L)
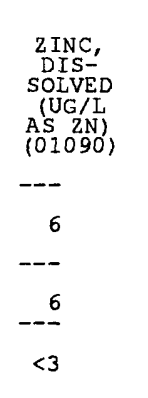

$--$

CARBON,

ORGANIC ORGANIC

DIS- PENDED

(MG/L (MG/L

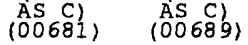

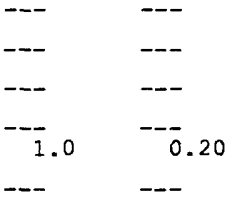

ETHO-
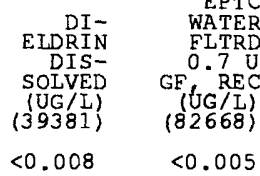

PROP

GFG/L)
$(82668)$

WATER $0.7 \mathrm{U}$

GF $(\mathrm{REC}$

PHORATE

WATER

GF REC

$\{82664\}$

$<0.011$

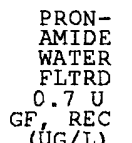

$<0.012$

$(U G / L)$
$(82676)$

$<0.009$

PRO-

PANIL

FLTRD

GF 0.7 REC

$(\mathrm{UG} / \mathrm{L})$
$(82679)$

$<0.016$

CARBO-

$\begin{aligned} \text { BUTYL- } & \text { FURAN } \\ \text { ATE, } & \text { WATER } \\ \text { WATER, } & \text { FLTRD }\end{aligned}$

FLTRD

GF, REC

(U2674)

CYANA-

$\begin{array}{rr}\text { WATER' } & \text { P PD' } \\ \text { DISS', } & \text { DDE } \\ \text { REC } & \text { DISSOLV } \\ (\text { UG } & (\mathrm{UG} / \mathrm{L}) \\ (04041) & (34653)\end{array}$

$<0.013$

$<0.013$

$<0.020$ 
IIIINOIS RIVER SUBBASIN

07197000 BARON FORK AT EIDON, OKIAHOMA--CONTINUED

PESTICIDE ANALYSES, WATER YEAR OCTOBER 1994 TO SEPTEMBER 1995

\begin{tabular}{|c|c|c|c|c|c|c|c|c|c|c|}
\hline DATE & $\begin{array}{r}\text { DIAZ- } \\
\text { INON } \\
\text { D10 SRG } \\
\text { WAT FLT } \\
0.7 \mathrm{U} \\
\text { GF REC } \\
\text { PERCENT } \\
(91063)\end{array}$ & $\begin{array}{c}2,6-D I- \\
\text { ETHYL } \\
\text { ANILINE } \\
\text { WAT FLT } \\
0.7 \mathrm{U} \\
\text { GE REC } \\
(\mathrm{UG} / \mathrm{L}) \\
(82660)\end{array}$ & $\begin{array}{c}\text { DISUL- } \\
\text { FOTON } \\
\text { WATER } \\
\text { FLTRD } \\
0.7 \mathrm{UU} \\
\text { GE REC } \\
(\mathrm{UG} / \mathrm{L}) \\
(82677)\end{array}$ & $\begin{array}{r}\text { ETHAL- } \\
\text { FLUR- } \\
\text { ALIN } \\
\text { WAT FLT } \\
0.7 \text { U } \\
\text { GF REC } \\
(\text { UG } \\
(8266)\end{array}$ & $\begin{array}{r}\text { FONOFOS } \\
\text { WATER } \\
\text { DISS } \\
\text { REC } \\
(\text { UG } / \mathrm{L}) \\
(04095)\end{array}$ & $\begin{array}{r}\text { HCH } \\
\text { ALPHA } \\
\text { D6 SRG } \\
\text { WAT FLT } \\
0.7 \mathrm{~T} \\
\text { GF, REC } \\
\text { PERCENT } \\
(91065)\end{array}$ & $\begin{array}{r}\text { LIN- } \\
\text { URON } \\
\text { WATER } \\
\text { FLTRD } \\
0.7 \mathrm{U} \\
\text { GE, REC } \\
(\mathrm{UG} / \mathrm{L}) \\
(82666)\end{array}$ & $\begin{array}{r}\text { METHYL } \\
\text { PARA- } \\
\text { THION } \\
\text { WAT FLT } \\
0.7 \mathrm{U} \\
\text { GE REC } \\
(\mathrm{UG} / \mathrm{L}) \\
(82667)\end{array}$ & $\begin{array}{c}\text { METRI- } \\
\text { BUZIN } \\
\text { SENCOR } \\
\text { WATER } \\
\text { DISSOLV } \\
\text { (UG/L) } \\
(82630)\end{array}$ & $\begin{array}{r}\text { NAPROP- } \\
\text { AMIDE } \\
\text { WATER } \\
\text { FLTRD } \\
0.7 \mathrm{~T} \\
\text { GF, REC } \\
(\text { UG/L) } \\
(82684)\end{array}$ \\
\hline $\begin{array}{l}\text { MAY } 1995 \\
15 \ldots\end{array}$ & 34.9 & $<0.006$ & $<0.060$ & $<0.013$ & $<0.008$ & 35.3 & $<0.039$ & $<0.035$ & $<0.012$ & $<0.010$ \\
\hline DATE & $\begin{array}{r}\text { PEB- } \\
\text { UIATE } \\
\text { WATER } \\
\text { FILTRD } \\
0.7 \mathrm{U} \\
\text { GF REC } \\
(\mathrm{UG} / \mathrm{L}) \\
(82669)\end{array}$ & $\begin{array}{r}\text { PER- } \\
\text { METHRIN } \\
\text { CIS } \\
\text { WAT FLT } \\
0.7 \text { U } \\
\text { GF REC } \\
\left(\begin{array}{l}\text { UG } / L\} \\
(82687\end{array}\right.\end{array}$ & $\begin{array}{c}\text { PRO- } \\
\text { METON, } \\
\text { WATER, } \\
\text { DISSS' } \\
\text { REC } \\
(\text { UG/L) } \\
(04037)\end{array}$ & $\begin{array}{c}\text { PROP- } \\
\text { CHLOR, } \\
\text { WATER, } \\
\text { DISS' } \\
\text { RE\& } \\
(\text { UG/L) } \\
(04024)\end{array}$ & $\begin{array}{r}\text { PRO- } \\
\text { PARGITE } \\
\text { WATER } \\
\text { FLTRD } \\
0.7 \mathrm{U} \\
\text { GF REC } \\
(\mathrm{UG} / \mathrm{L}) \\
(82685)\end{array}$ & $\begin{array}{r}\text { TEBU- } \\
\text { THIURON } \\
\text { WATER } \\
\text { FLTRD } \\
0.7 \text { U } \\
\text { GE, REC } \\
\text { (UG/L) } \\
(82670)\end{array}$ & $\begin{array}{r}\text { TER- } \\
\text { BUFOS } \\
\text { WATER } \\
\text { FLTRD } \\
0.7 \mathrm{U} \\
\text { GE }(\text { REC } \\
(\text { UG/L) } \\
(82675)\end{array}$ & $\begin{array}{r}\text { THIO- } \\
\text { BENCARB } \\
\text { WATER } \\
\text { FLTRD } \\
0.7 \text { U } \\
\text { GF REC } \\
(\text { UG/L) } \\
(82681)\end{array}$ & $\begin{array}{r}\text { TRI- } \\
\text { FLUR- } \\
\text { ALIN } \\
\text { WAT FLT } \\
0.7 \text { TU } \\
\text { GF } \mathrm{REC} \\
(8 \mathrm{UG} / \mathrm{II}) \\
(82661)\end{array}$ & \\
\hline $15 \ldots$ & $<0.009$ & $<0.016$ & $<0.008$ & $<0.015$ & $<0.006$ & $E 0.010$ & $<0.012$ & $<0.008$ & $<0.012$ & \\
\hline
\end{tabular}


PERIOD OF RECORD.--Water years 1948, 1952, 1954 to current year.

PERTOD OF DAILY RECORD.

SPECIFIC CONDUCTANCE: October 1947 to September 1948, October 1953 to September 1963.

WRATER TEFPRRATURE: October 1947 to September 1948, October 1953 to September 1963, October 1992 to September 1993. REMaRs.--Specific conductance, $\mathrm{pH}$, water temperature, dissolved oxygen, and alkalinity were determined in the fleld. Prior to October 1992 records of continuous water temperature were collected $4.2 \mathrm{ml}$ upstream.

EXIREMIES FOR PERICD OF DAIIYY RECORD.--

SPECIFIC CONDUCTANCE: Maximum daily 396 microslemens, Aug. 12, 1956; minimum daliy 123 microslemens, July $14,1957$. WATER TEMPERATURE: Maximum $24.0^{\circ} \mathrm{C}$, Sept. 28-30, Oct. 1, 2, 1958, Aug. 29, 1993; minimum $4.5^{\circ} \mathrm{C}$ several days in winter months.

EXUTREMIES FOR CURRENT YEAR.--

WATER TERPERATURE: Maximum $22.0^{\circ} \mathrm{C}$, Aug. 14, 31, Sept. 17; minimum $5.0^{\circ} \mathrm{C}$, Jan. 8.

WATER-QUALITY DATA, WATER YEAR OCTOBER 1994 TO SEPTEMBER 1995

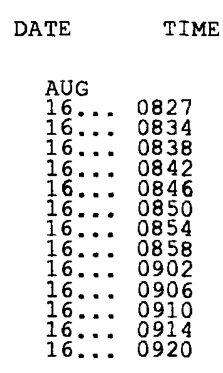

DATE TIME

$\begin{array}{ll}\mathrm{OCT} \\ 18.1 & 1040\end{array}$

DEC .. 1645

FEB 13.1630

APR . . 0900

MAY 1000

JUN 10.1000

21... 1330
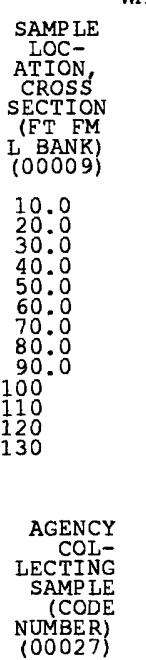

1028

1028

1028

1028

1028

1028

OXYGEN,

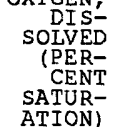

$\begin{array}{lll}\text { DATE } & \text { SOLVED } & \text { SATUR- } \\ & (M G / L) & \text { ATION) } \\ \text { OCT } & (00300) & (00301)\end{array}$

$\begin{array}{lll}\text { OCT } & 6.7 & 70\end{array}$

$07 . . \quad 8.4$

FEB 13.2

APR $\quad 10.8$

MAY

गुN

$$
7.8
$$

5.1

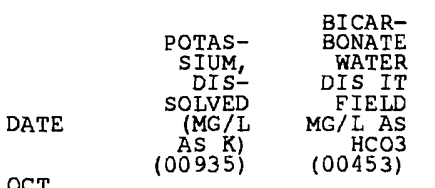

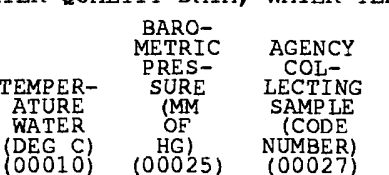
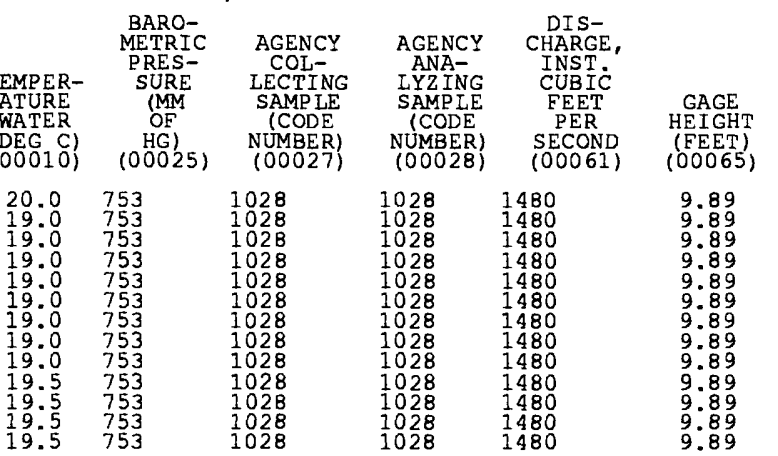

1480
1480
1480
1480
1480
1480
1480
1480
1480
1480
1480
1480
1480
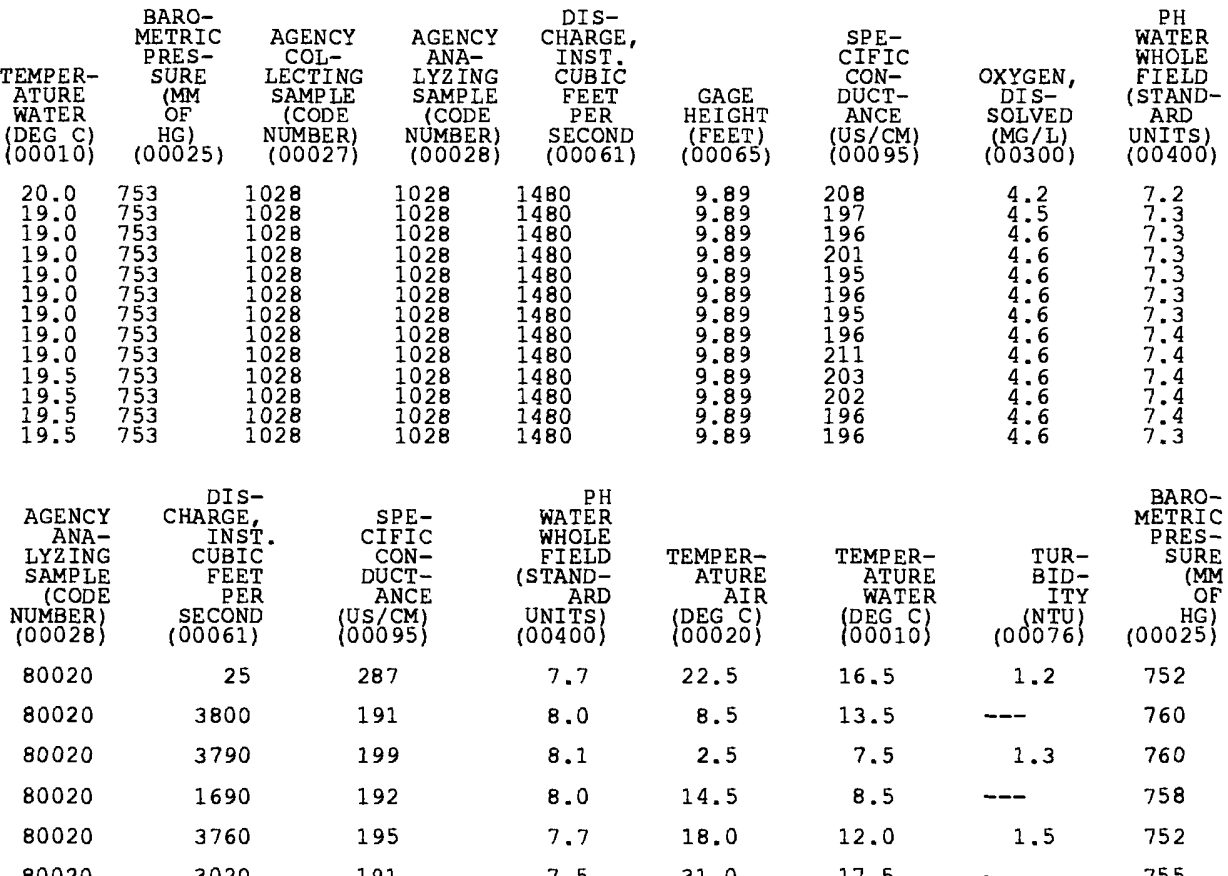

TEMPER-
ATURE
AIR
$\left(\begin{array}{l}D E G C \\ 00020\end{array}\right)$

80020

80020

80020

80020

80020

80020

25
3800
3790
1690
3760
3020

7.7

8.0

8.1

8.0

7.7

7.5

22.5

8.5

2.5

14.5

18.0

31.0

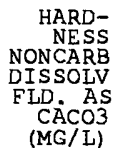

AS N)
$(00602)$

0.85

--- ---

--- 79

1.3
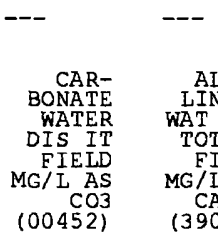

0

0

0

0

0

0
84

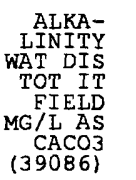

19

$---$

4

10

---

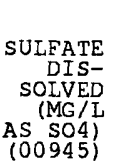

7.0

67

75

74

74

61

$---$

$--$

7.0
$(00904)$

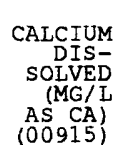

39

---

29

---

31

$---$

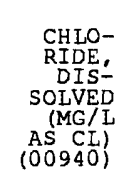

29

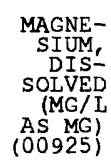

2.3

$--$

1.6

1.6$$
-
$$$$
6
$$

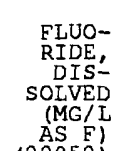

AS F
$(00950)$

$<0.10$

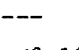

$<0.10$

-
TEMPER-
ATURE
WATER
$\left(\begin{array}{c}D E G \text { C } \\ 00010\end{array}\right)$

16.5

13.5

7.5

8.5

12.0

17.5

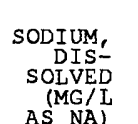

AS NA)
$(00930)$

16

---

4.5

4.5

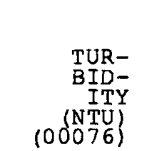

BARO-

METRIC

SURE

OF

1.2752

-- 760

$1.3 \quad 760$

-- 758

$1.5 \quad 752$

$--\quad 755$ 
IIIINOIS RTVER SUBBASIN

07198000 ILLINOIS RIVER NEAR GORE, OKLAHOMA--CONTINUED WATER-QUALITY DATA, WATER YEAR OCTOBER 1994 TO SEPTEMBER 1995

\begin{tabular}{|c|c|c|c|c|c|c|c|c|c|c|}
\hline DATE & $\begin{array}{r}\text { SOLIDS, } \\
\text { DISS } \\
\text { SOLVED } \\
\text { (TONS } \\
\text { PER } \\
\text { DAY) } \\
\{70302\}\end{array}$ & $\begin{array}{c}\text { NITRO- } \\
\text { GEN } \\
\text { NITRATE } \\
\text { TOTAL } \\
\text { (MG/L } \\
\text { AS N) } \\
(00620)\end{array}$ & $\begin{array}{r}\text { NITRO- } \\
\text { GEN } \\
\text { NITRATE } \\
\text { DIS } \\
\text { SOLVED } \\
\text { (MG/L } \\
\text { AS N) } \\
(00618)\end{array}$ & $\begin{array}{c}\text { NITRO- } \\
\text { GEN } \\
\text { NITRATE } \\
\text { DIS- } \\
\text { SOIVED } \\
\text { (MG/I } \\
\text { AS NO3) } \\
\text { (71851) }\end{array}$ & $\begin{array}{r}\text { NITRO- } \\
\text { GEN } \\
\text { NITRITE } \\
\text { DIS- } \\
\text { SOLVED } \\
\text { (MG / } \\
\text { AS N } \\
(00613)\end{array}$ & $\begin{array}{r}\text { NITRO- } \\
\text { GEN, } \\
\text { NITRITE } \\
\text { DIS- } \\
\text { SOLVED } \\
\text { (MG/I } \\
\text { AS NO2) } \\
(71856)\end{array}$ & $\begin{array}{c}\text { NITRO- } \\
\text { GEN } \\
\text { NO2+NO\} } \\
\text { TOTAI } \\
\text { (MG/L } \\
\text { AS N) } \\
(00630\}\end{array}$ & $\begin{array}{c}\text { NITRO- } \\
\text { GEN } \\
\text { NO2+NO3 } \\
\text { DIS- } \\
\text { SOIVED } \\
\text { (MG/I } \\
\text { AS N }) \\
(00631\}\end{array}$ & $\begin{array}{c}\text { NITRO- } \\
\text { GEN } \\
\text { AMMONIA } \\
\text { DIS- } \\
\text { SOLVED } \\
\text { (MG/I } \\
\text { AS N) } \\
(00608)\end{array}$ & $\begin{array}{c}\text { NITRO- } \\
\text { GEN } \\
\text { AMMONIA } \\
\text { DIS- } \\
\text { SOLVED } \\
\text { (MG/I } \\
\text { AS NH4) } \\
(71846)\end{array}$ \\
\hline \multirow{6}{*}{$\begin{array}{l}\text { OCT } \\
18 . \\
\text { DEC } \\
07 . \\
\text { FEB } \\
13 \\
\text { AP } \\
04 \\
04 \\
\text { MAY } \\
10 \\
\text { JUN } \\
21 \ldots \\
21 \ldots\end{array}$} & 10.7 & 0.520 & 0.520 & 2.3 & 0.030 & 0.10 & 0.550 & 0.550 & 0.160 & 0.21 \\
\hline & -- & 0.360 & 0.360 & 1.6 & 0.010 & 0.03 & 0.370 & 0.370 & $<0.015$ & -- \\
\hline & 1060 & 0.590 & --- & -- & $<0.010$ & -- & 0.590 & 0.590 & $<0.015$ & -- \\
\hline & -- & 0.840 & 0.840 & 3.7 & 0.020 & 0.07 & 0.860 & 0.860 & $<0.015$ & -- \\
\hline & 1100 & 1.09 & 1.09 & 4.8 & 0.010 & 0.03 & 1.10 & 1.10 & 0.020 & 0.03 \\
\hline & -- & 0.990 & 0.990 & 4.4 & 0.010 & 0.03 & 1.00 & 1.00 & 0.050 & 0.06 \\
\hline DATE & $\begin{array}{c}\text { NITRO- } \\
\text { GEN } \\
\text { ORGANIC } \\
\text { TOTAL } \\
\text { (MG/L } \\
\text { AS N) } \\
(00605)\end{array}$ & $\begin{array}{c}\text { NITRO- } \\
\text { GEN, } \\
\text { ORGANIC } \\
\text { DIS- } \\
\text { SOLVED } \\
\text { (MG/L } \\
\text { AS N } \\
(00607)\end{array}$ & $\begin{array}{c}\text { NITRO- } \\
\text { GEN AM- } \\
\text { MONIA + } \\
\text { ORGANIC } \\
\text { TOTAL } \\
\text { (MG / } \\
\text { AS N) } \\
(00625)\end{array}$ & $\begin{array}{c}\text { NITRO- } \\
\text { GEN AM- } \\
\text { MONIA }+ \\
\text { ORGANIC } \\
\text { DIS. } \\
\text { (MG } \\
\text { AS N } \\
(00623)\end{array}$ & $\begin{array}{r}\text { NITRO- } \\
\text { GEN, } \\
\text { TOTAL } \\
\text { (MG/L } \\
\text { AS N) } \\
(00600)\end{array}$ & $\begin{array}{c}\text { PHOS- } \\
\text { PHORUS } \\
\text { TOTAL } \\
\text { (MG/I } \\
\text { AS P) } \\
(00665)\end{array}$ & $\begin{array}{c}\text { PHOS- } \\
\text { PHORUS } \\
\text { DIS- } \\
\text { SOLVED } \\
\text { (MG/L } \\
\text { AS P) } \\
(00666)\end{array}$ & $\begin{array}{c}\text { PHOS- } \\
\text { PHORUS } \\
\text { ORTHO, } \\
\text { DIS - } \\
\text { SOIVED } \\
\text { (MG/I } \\
\text { AS P) } \\
(00671)\end{array}$ & $\begin{array}{c}\text { PHOS- } \\
\text { PHATE, } \\
\text { ORTHO, } \\
\text { DIS- } \\
\text { SOLVED } \\
\text { (MG/ } \\
\text { AS PO4) } \\
(00660)\end{array}$ & \\
\hline $18 \ldots$ & 0.24 & 0.14 & 0.40 & 0.30 & 0.95 & 0.030 & 0.030 & 0.020 & 0.06 & \\
\hline $07 \ldots$ & -- & -- & $<0.20$ & $<0.20$ & --- & 0.020 & 0.030 & 0.020 & 0.06 & \\
\hline $13 \ldots$ & -- & -- & $<0.20$ & $<0.20$ & -- & $<0.010$ & $<0.010$ & $<0.010$ & -- & \\
\hline $04 \dot{Y}^{\circ}$ & -- & -- & $<0.20$ & $<0.20$ & -- & $<0.010$ & $<0.010$ & $<0.010$ & -- & \\
\hline $10 \ldots$ & 0.28 & 0.18 & 0.30 & 0.20 & 1.4 & 0.020 & 0.010 & 0.020 & 0.06 & \\
\hline $21 \ldots$ & -- & -- & $<0.20$ & $<0.20$ & --- & 0.040 & 0.040 & 0.050 & 0.15 & \\
\hline
\end{tabular}

WATER TEMPERATURE, DEGREES CELSIUS, WATER YEAR OCTOBER 1994 TO SEPTEMBER 1995

\begin{tabular}{|c|c|c|c|c|c|c|c|c|c|c|c|c|}
\hline DAY & MAX & $\begin{array}{c}\text { MIN } \\
\text { OCTOBER }\end{array}$ & MEAN & MAX & $\begin{array}{c}\text { MIN } \\
\text { NOVEMBER }\end{array}$ & MEAN & MAX & $\begin{array}{c}\text { MIN } \\
\text { DECEMBER }\end{array}$ & MEAN & MAX & $\begin{array}{c}\text { MIN } \\
\text { JANUARY }\end{array}$ & MEAN \\
\hline $\begin{array}{l}1 \\
2 \\
3 \\
4 \\
5\end{array}$ & $\begin{array}{l}18.0 \\
20.0 \\
16.5 \\
17.5 \\
-\end{array}$ & $\begin{array}{l}15.0 \\
15.0 \\
20.5 \\
15.0 \\
-\end{array}$ & $\begin{array}{l}16.0 \\
16.5 \\
15.5 \\
16.0 \\
-\end{array}$ & $\begin{array}{l}14.0 \\
14.5 \\
15.0 \\
16.0 \\
15.5\end{array}$ & $\begin{array}{r}9.0 \\
11.5 \\
14.5 \\
14.5 \\
15.0\end{array}$ & $\begin{array}{l}12.0 \\
13.0 \\
14.5 \\
15.0 \\
15.0\end{array}$ & $\begin{array}{l}14.0 \\
14.0 \\
14.0 \\
14.0 \\
14.0\end{array}$ & $\begin{array}{l}12.5 \\
12.5 \\
13.0 \\
13.0 \\
13.0\end{array}$ & $\begin{array}{l}13.5 \\
13.5 \\
13.5 \\
13.5 \\
13.5\end{array}$ & $\begin{array}{l}11.0 \\
11.0 \\
10.5 \\
10.5 \\
10.0\end{array}$ & $\begin{array}{l}8.0 \\
8.0 \\
9.0 \\
8.0 \\
7.5\end{array}$ & $\begin{array}{r}10.0 \\
10.0 \\
10.0 \\
9.5 \\
9.0\end{array}$ \\
\hline $\begin{array}{r}6 \\
7 \\
8 \\
9 \\
10\end{array}$ & $\begin{array}{l}-17.0 \\
16.0 \\
17.5 \\
17.0\end{array}$ & $\begin{array}{l}--\overline{15.0} \\
14.5 \\
13.0 \\
12.0\end{array}$ & $\begin{array}{l}-16.0 \\
15.5 \\
15.5 \\
14.0\end{array}$ & $\begin{array}{l}15.0 \\
15.0 \\
15.0 \\
15.5 \\
15.5\end{array}$ & $\begin{array}{l}14.5 \\
13.5 \\
15.0 \\
15.0 \\
15.0\end{array}$ & $\begin{array}{l}14.5 \\
14.5 \\
15.0 \\
15.0 \\
15.5\end{array}$ & $\begin{array}{l}14.0 \\
14.0 \\
13.5 \\
13.5 \\
13.0\end{array}$ & $\begin{array}{l}13.0 \\
13.0 \\
12.5 \\
11.5 \\
11.5\end{array}$ & $\begin{array}{l}13.5 \\
13.5 \\
13.0 \\
12.5 \\
12.5\end{array}$ & $\begin{array}{r}10.0 \\
10.0 \\
8.5 \\
10.0 \\
11.0\end{array}$ & $\begin{array}{l}8.0 \\
7.0 \\
5.0 \\
5.5 \\
8.5\end{array}$ & $\begin{array}{l}9.5 \\
8.5 \\
7.0 \\
8.0 \\
9.5\end{array}$ \\
\hline $\begin{array}{l}11 \\
12 \\
13 \\
14 \\
15\end{array}$ & $\begin{array}{l}17.0 \\
15.5 \\
16.0 \\
15.5 \\
16.0\end{array}$ & $\begin{array}{l}13.0 \\
13.5 \\
14.5 \\
14.0 \\
14.5\end{array}$ & $\begin{array}{l}15.0 \\
14.5 \\
15.5 \\
14.5 \\
15.0\end{array}$ & $\begin{array}{l}15.5 \\
15.5 \\
15.5 \\
15.5 \\
15.5\end{array}$ & $\begin{array}{l}15.0 \\
15.0 \\
15.0 \\
15.5 \\
15.5\end{array}$ & $\begin{array}{l}15.5 \\
15.5 \\
15.5 \\
15.5 \\
15.5\end{array}$ & $\begin{array}{l}12.5 \\
12.5 \\
12.5 \\
12.0 \\
12.0\end{array}$ & $\begin{array}{l}12.5 \\
12.5 \\
12.0 \\
12.0 \\
12.0\end{array}$ & $\begin{array}{l}12.5 \\
12.5 \\
12.0 \\
12.0 \\
12.0\end{array}$ & $\begin{array}{l}11.0 \\
10.5 \\
10.5 \\
10.0 \\
10.0\end{array}$ & $\begin{array}{l}8.5 \\
9.5 \\
8.5 \\
8.0 \\
9.0\end{array}$ & $\begin{array}{r}10.0 \\
10.0 \\
9.5 \\
9.5 \\
9.5\end{array}$ \\
\hline $\begin{array}{l}16 \\
17 \\
18 \\
19 \\
20\end{array}$ & $\begin{array}{l}17.5 \\
17.5 \\
17.5 \\
18.0 \\
18.5\end{array}$ & $\begin{array}{l}15.0 \\
15.5 \\
15.5 \\
16.0 \\
15.5\end{array}$ & $\begin{array}{l}16.5 \\
16.5 \\
17.0 \\
16.5 \\
17.0\end{array}$ & $\begin{array}{l}15.5 \\
15.5 \\
15.5 \\
15.5 \\
15.5\end{array}$ & $\begin{array}{l}15.0 \\
15.0 \\
15.0 \\
15.5 \\
15.0\end{array}$ & $\begin{array}{l}15.5 \\
15.5 \\
15.5 \\
15.5 \\
15.5\end{array}$ & $\begin{array}{l}12.0 \\
12.0 \\
12.0 \\
12.0 \\
13.0\end{array}$ & $\begin{array}{l}12.0 \\
12.0 \\
11.5 \\
11.5 \\
11.5\end{array}$ & $\begin{array}{l}12.0 \\
12.0 \\
12.0 \\
11.5 \\
11.5\end{array}$ & $\begin{array}{r}10.0 \\
9.5 \\
9.0 \\
9.0 \\
9.0\end{array}$ & $\begin{array}{l}8.5 \\
8.5 \\
8.5 \\
8.5 \\
8.5\end{array}$ & $\begin{array}{l}9.5 \\
9.0 \\
9.0 \\
8.5 \\
8.5\end{array}$ \\
\hline $\begin{array}{l}21 \\
22 \\
23 \\
24 \\
25\end{array}$ & $\begin{array}{l}17.5 \\
17.5 \\
18.0 \\
17.0 \\
16.0\end{array}$ & $\begin{array}{l}15.5 \\
15.5 \\
13.5 \\
14.0 \\
13.5\end{array}$ & $\begin{array}{l}16.5 \\
16.0 \\
15.5 \\
15.0 \\
15.0\end{array}$ & $\begin{array}{l}15.5 \\
15.0 \\
15.0 \\
15.0 \\
14.5\end{array}$ & $\begin{array}{l}15.0 \\
15.0 \\
14.5 \\
13.5 \\
14.0\end{array}$ & $\begin{array}{l}15.0 \\
15.0 \\
15.0 \\
14.5 \\
14.5\end{array}$ & $\begin{array}{l}13.0 \\
12.5 \\
12.5 \\
12.0 \\
11.5\end{array}$ & $\begin{array}{l}11.5 \\
11.0 \\
10.5 \\
10.0 \\
10.0\end{array}$ & $\begin{array}{l}12.0 \\
11.5 \\
11.5 \\
11.0 \\
11.0\end{array}$ & $\begin{array}{l}8.5 \\
8.5 \\
8.5 \\
8.5 \\
8.5\end{array}$ & $\begin{array}{l}8.5 \\
8.5 \\
8.0 \\
8.0 \\
8.0\end{array}$ & $\begin{array}{l}8.5 \\
8.5 \\
8.5 \\
8.5 \\
8.5\end{array}$ \\
\hline $\begin{array}{l}26 \\
27 \\
28 \\
29 \\
30 \\
31\end{array}$ & $\begin{array}{l}15.0 \\
15.0 \\
15.5 \\
16.5 \\
14.5 \\
14.5\end{array}$ & $\begin{array}{l}12.5 \\
11.5 \\
11.0 \\
10.5 \\
12.0 \\
11.0\end{array}$ & $\begin{array}{l}14.5 \\
14.0 \\
13.0 \\
13.5 \\
14.0 \\
13.0\end{array}$ & $\begin{array}{l}14.5 \\
15.0 \\
14.5 \\
14.5 \\
14.0 \\
--\end{array}$ & $\begin{array}{l}14.0 \\
14.5 \\
12.5 \\
13.5 \\
13.0 \\
--\end{array}$ & $\begin{array}{l}14.5 \\
14.5 \\
14.0 \\
14.0 \\
14.0 \\
--\end{array}$ & $\begin{array}{l}11.5 \\
11.5 \\
11.5 \\
11.5 \\
11.5 \\
11.0\end{array}$ & $\begin{array}{r}9.5 \\
9.5 \\
10.5 \\
10.0 \\
9.5 \\
10.0\end{array}$ & $\begin{array}{l}11.0 \\
10.5 \\
11.0 \\
10.5 \\
10.5 \\
10.5\end{array}$ & $\begin{array}{l}10.0 \\
10.5 \\
10.5 \\
10.0 \\
10.0 \\
10.0\end{array}$ & $\begin{array}{l}8.0 \\
8.0 \\
8.5 \\
8.0 \\
8.0 \\
7.5\end{array}$ & $\begin{array}{l}8.5 \\
9.0 \\
9.0 \\
8.5 \\
8.5 \\
8.5\end{array}$ \\
\hline NTH & --- & --- & --- & 16.0 & 9.0 & 14.8 & 14.0 & 9.5 & 12.0 & 11.0 & 5.0 & 9.0 \\
\hline
\end{tabular}


IIUINOIS RTVER SUBBASIN

07198000 ILLINOIS RIVER NEAR GORE, OKIAAOMA--CONTINUED

WATER TEMPERATURE, DEGREES CELSIUS, WATER YEAR OCTOBER 1994 TO SEPTEMBER 1995

\begin{tabular}{|c|c|c|c|c|c|c|c|c|c|c|c|c|}
\hline DAY & MAX & $\begin{array}{c}\text { MIN } \\
\text { FEBRUARY }\end{array}$ & MEAN & MAX & $\begin{array}{c}\text { MIN } \\
\text { MARCH }\end{array}$ & MEAN & MAX & $\begin{array}{r}\text { MIN } \\
\text { APRIL }\end{array}$ & MEAN & MAX & $\begin{array}{l}\text { MIN } \\
\text { MAY }\end{array}$ & MEAN \\
\hline $\begin{array}{l}1 \\
2 \\
3 \\
4 \\
5\end{array}$ & $\begin{array}{l}9.5 \\
9.5 \\
9.5 \\
9.5 \\
9.5\end{array}$ & $\begin{array}{l}7.5 \\
8.0 \\
8.0 \\
7.0 \\
7.0\end{array}$ & $\begin{array}{l}8.5 \\
8.5 \\
8.5 \\
B .5 \\
B .5\end{array}$ & $\begin{array}{l}9.5 \\
9.5 \\
7.5 \\
9.0 \\
9.0\end{array}$ & $\begin{array}{l}7.0 \\
6.0 \\
7.5 \\
7.0 \\
7.5\end{array}$ & $\begin{array}{l}8.5 \\
7.5 \\
7.5 \\
8.0 \\
8.0\end{array}$ & $\begin{array}{l}10.0 \\
10.0 \\
10.0 \\
10.0 \\
10.5\end{array}$ & $\begin{array}{l}8.5 \\
8.0 \\
8.0 \\
8.5 \\
8.5\end{array}$ & $\begin{array}{l}9.0 \\
9.0 \\
9.0 \\
9.0 \\
9.5\end{array}$ & $\begin{array}{l}10.5 \\
11.0 \\
11.0 \\
11.0 \\
11.0\end{array}$ & $\begin{array}{l}10.5 \\
10.5 \\
10.5 \\
10.5 \\
10.5\end{array}$ & $\begin{array}{l}10.5 \\
10.5 \\
10.5 \\
11.0 \\
11.0\end{array}$ \\
\hline $\begin{array}{r}6 \\
7 \\
8 \\
9 \\
10\end{array}$ & $\begin{array}{l}9.0 \\
8.0 \\
8.0 \\
8.0 \\
8.0\end{array}$ & $\begin{array}{l}7.0 \\
7.5 \\
7.5 \\
7.5 \\
7.5\end{array}$ & $\begin{array}{l}8.0 \\
8.0 \\
7.5 \\
7.5 \\
7.5\end{array}$ & $\begin{array}{l}9.0 \\
9.0 \\
7.5 \\
7.5 \\
8.0\end{array}$ & $\begin{array}{l}7.5 \\
7.0 \\
7.0 \\
7.0 \\
7.0\end{array}$ & $\begin{array}{l}8.5 \\
7.5 \\
7.0 \\
7.0 \\
7.0\end{array}$ & $\begin{array}{l}10.5 \\
11.0 \\
13.5 \\
17.0 \\
16.5\end{array}$ & $\begin{array}{r}9.0 \\
8.5 \\
10.0 \\
13.5 \\
10.0\end{array}$ & $\begin{array}{r}9.5 \\
10.0 \\
12.0 \\
15.0 \\
13.0\end{array}$ & $\begin{array}{l}12.5 \\
13.5 \\
14.5 \\
14.5 \\
12.0\end{array}$ & $\begin{array}{l}11.0 \\
12.0 \\
13.0 \\
12.0 \\
11.5\end{array}$ & $\begin{array}{l}11.5 \\
12.0 \\
13.5 \\
13.0 \\
12.0\end{array}$ \\
\hline $\begin{array}{l}11 \\
12 \\
13 \\
14 \\
15\end{array}$ & $\begin{array}{l}8.0 \\
7.5 \\
7.5 \\
7.0 \\
7.0\end{array}$ & $\begin{array}{l}7.5 \\
7.0 \\
7.0 \\
7.0 \\
7.0\end{array}$ & $\begin{array}{l}7.5 \\
7.5 \\
7.0 \\
7.0 \\
7.0\end{array}$ & $\begin{array}{l}7.5 \\
7.5 \\
7.5 \\
8.0 \\
8.0\end{array}$ & $\begin{array}{l}7.0 \\
7.0 \\
7.0 \\
7.5 \\
7.5\end{array}$ & $\begin{array}{l}7.5 \\
7.5 \\
7.5 \\
7.5 \\
8.0\end{array}$ & $\begin{array}{r}11.5 \\
11.5 \\
9.5 \\
9.5 \\
9.0\end{array}$ & $\begin{array}{l}9.5 \\
9.0 \\
9.0 \\
9.0 \\
9.0\end{array}$ & $\begin{array}{r}10.5 \\
9.5 \\
9.0 \\
9.0 \\
9.0\end{array}$ & $\begin{array}{l}12.0 \\
12.0 \\
12.0 \\
12.5 \\
13.0\end{array}$ & $\begin{array}{l}11.5 \\
11.5 \\
11.5 \\
12.0 \\
12.0\end{array}$ & $\begin{array}{l}12.0 \\
12.0 \\
12.0 \\
12.0 \\
12.5\end{array}$ \\
\hline $\begin{array}{l}16 \\
17 \\
18 \\
19 \\
20\end{array}$ & $\begin{array}{r}7.0 \\
10.0 \\
10.0 \\
9.0 \\
9.0\end{array}$ & $\begin{array}{l}7.0 \\
7.0 \\
7.0 \\
7.0 \\
7.0\end{array}$ & $\begin{array}{l}7.0 \\
7.5 \\
8.0 \\
8.0 \\
8.0\end{array}$ & $\begin{array}{r}8.0 \\
10.5 \\
11.5 \\
12.5 \\
11.0\end{array}$ & $\begin{array}{l}8.0 \\
7.5 \\
9.5 \\
8.5 \\
8.0\end{array}$ & $\begin{array}{r}8.0 \\
9.0 \\
10.5 \\
10.5 \\
9.0\end{array}$ & $\begin{array}{r}9.5 \\
11.5 \\
11.5 \\
11.5 \\
11.5\end{array}$ & $\begin{array}{l}9.0 \\
9.0 \\
9.5 \\
9.0 \\
9.5\end{array}$ & $\begin{array}{r}9.0 \\
9.5 \\
10.5 \\
10.0 \\
10.5\end{array}$ & $\begin{array}{l}13.0 \\
13.0 \\
13.5 \\
14.0 \\
14.0\end{array}$ & $\begin{array}{l}12.5 \\
13.0 \\
13.0 \\
13.5 \\
13.5\end{array}$ & $\begin{array}{l}13.0 \\
13.0 \\
13.0 \\
13.5 \\
14.0\end{array}$ \\
\hline $\begin{array}{l}21 \\
22 \\
23 \\
24 \\
25\end{array}$ & $\begin{array}{l}9.0 \\
9.0 \\
9.5 \\
9.5 \\
9.5\end{array}$ & $\begin{array}{l}7.0 \\
8.0 \\
8.5 \\
8.0 \\
8.0\end{array}$ & $\begin{array}{l}8.5 \\
8.5 \\
9.0 \\
9.0 \\
9.0\end{array}$ & $\begin{array}{l}10.5 \\
10.0 \\
10.0 \\
10.0 \\
10.0\end{array}$ & $\begin{array}{l}8.0 \\
8.0 \\
8.0 \\
8.0 \\
8.0\end{array}$ & $\begin{array}{l}9.0 \\
9.0 \\
9.0 \\
8.5 \\
9.0\end{array}$ & $\begin{array}{l}11.5 \\
10.0 \\
10.5 \\
10.0 \\
10.0\end{array}$ & $\begin{array}{r}9.5 \\
9.5 \\
10.0 \\
9.5 \\
9.5\end{array}$ & $\begin{array}{l}10.0 \\
10.0 \\
10.0 \\
10.0 \\
10.0\end{array}$ & $\begin{array}{l}14.0 \\
14.5 \\
14.5 \\
15.0 \\
15.0\end{array}$ & $\begin{array}{l}14.0 \\
14.0 \\
14.0 \\
14.5 \\
14.0\end{array}$ & $\begin{array}{l}14.0 \\
14.0 \\
14.5 \\
14.5 \\
14.5\end{array}$ \\
\hline $\begin{array}{r}26 \\
27 \\
28 \\
29 \\
30 \\
31 \\
\text { MONTH }\end{array}$ & $\begin{array}{r}9.0 \\
11.0 \\
10.5 \\
\hdashline- \\
\hdashline 11.0\end{array}$ & $\begin{array}{c}8.5 \\
8.5 \\
8.5 \\
-- \\
-- \\
-7.0\end{array}$ & $\begin{array}{c}9.0 \\
9.5 \\
9.5 \\
--- \\
--- \\
-8.1\end{array}$ & $\begin{array}{l}10.0 \\
10.0 \\
10.0 \\
10.0 \\
10.0 \\
10.0 \\
12.5\end{array}$ & $\begin{array}{l}8.5 \\
8.0 \\
8.0 \\
8.5 \\
8.5 \\
9.0 \\
6.0\end{array}$ & $\begin{array}{l}9.0 \\
9.0 \\
9.0 \\
9.0 \\
9.0 \\
9.5 \\
8.4\end{array}$ & $\begin{array}{r}10.0 \\
10.0 \\
10.5 \\
10.5 \\
10.5 \\
-17.0\end{array}$ & $\begin{array}{r}9.5 \\
9.5 \\
10.0 \\
10.0 \\
10.0 \\
-8.0\end{array}$ & $\begin{array}{r}9.5 \\
10.0 \\
10.0 \\
10.0 \\
10.0 \\
-10.0\end{array}$ & $\begin{array}{l}14.5 \\
14.5 \\
15.0 \\
15.0 \\
15.0 \\
15.0 \\
15.0\end{array}$ & $\begin{array}{l}14.0 \\
14.5 \\
14.5 \\
14.5 \\
14.5 \\
15.0 \\
10.5\end{array}$ & $\begin{array}{l}14.5 \\
14.5 \\
15.0 \\
15.0 \\
15.0 \\
15.0 \\
13.0\end{array}$ \\
\hline DAY & MAX & $\begin{array}{l}\text { MIN } \\
\text { JUNE }\end{array}$ & MEAN & MAX & $\begin{array}{l}\text { MIN } \\
\text { JULY }\end{array}$ & MEAN & MAX & $\begin{array}{c}\text { MIN } \\
\text { AUGUST }\end{array}$ & MEAN & MAX & $\begin{array}{c}\text { MIN } \\
\text { SEPTEMBER }\end{array}$ & MEAN \\
\hline $\begin{array}{l}1 \\
2 \\
3 \\
4 \\
5\end{array}$ & $\begin{array}{l}15.5 \\
15.0 \\
15.5 \\
16.0 \\
17.0\end{array}$ & $\begin{array}{l}15.0 \\
14.5 \\
14.5 \\
14.5 \\
15.0\end{array}$ & $\begin{array}{l}15.0 \\
15.0 \\
15.0 \\
15.0 \\
15.5\end{array}$ & $\begin{array}{l}16.5 \\
16.5 \\
16.5 \\
16.5 \\
16.5\end{array}$ & $\begin{array}{l}16.0 \\
16.0 \\
16.0 \\
16.0 \\
16.0\end{array}$ & $\begin{array}{l}16.0 \\
16.0 \\
16.0 \\
16.5 \\
16.5\end{array}$ & $\begin{array}{l}18.0 \\
18.0 \\
18.0 \\
18.5 \\
18.0\end{array}$ & $\begin{array}{l}17.5 \\
17.5 \\
17.5 \\
17.5 \\
17.5\end{array}$ & $\begin{array}{l}17.5 \\
18.0 \\
18.0 \\
18.0 \\
18.0\end{array}$ & $\begin{array}{l}20.5 \\
20.0 \\
20.0 \\
20.5 \\
21.5\end{array}$ & $\begin{array}{l}19.5 \\
19.5 \\
19.0 \\
19.0 \\
19.5\end{array}$ & $\begin{array}{l}20.0 \\
19.5 \\
19.5 \\
19.5 \\
20.0\end{array}$ \\
\hline $\begin{array}{r}6 \\
7 \\
8 \\
9 \\
10\end{array}$ & $\begin{array}{l}16.0 \\
16.0 \\
16.5 \\
17.0 \\
18.5\end{array}$ & $\begin{array}{l}15.0 \\
15.0 \\
15.0 \\
15.5 \\
16.5\end{array}$ & $\begin{array}{l}15.5 \\
15.5 \\
15.5 \\
16.0 \\
17.5\end{array}$ & $\begin{array}{l}16.5 \\
16.5 \\
17.0 \\
17.0 \\
17.0\end{array}$ & $\begin{array}{l}16.0 \\
16.0 \\
16.5 \\
16.5 \\
16.5\end{array}$ & $\begin{array}{l}16.5 \\
16.5 \\
16.5 \\
16.5 \\
17.0\end{array}$ & $\begin{array}{l}18.0 \\
18.5 \\
19.0 \\
18.5 \\
18.5\end{array}$ & $\begin{array}{l}18.0 \\
18.0 \\
18.0 \\
18.0 \\
18.0\end{array}$ & $\begin{array}{l}18.0 \\
18.0 \\
18.0 \\
18.0 \\
18.0\end{array}$ & $\begin{array}{l}21.0 \\
21.0 \\
20.0 \\
19.0 \\
20.0\end{array}$ & $\begin{array}{l}19.5 \\
19.5 \\
19.0 \\
18.0 \\
17.5\end{array}$ & $\begin{array}{l}20.0 \\
20.0 \\
19.0 \\
18.5 \\
18.5\end{array}$ \\
\hline $\begin{array}{l}11 \\
12 \\
13 \\
14 \\
15\end{array}$ & $\begin{array}{l}17.0 \\
16.5 \\
19.5 \\
19.5 \\
20.5\end{array}$ & $\begin{array}{l}16.0 \\
16.0 \\
16.0 \\
16.5 \\
17.0\end{array}$ & $\begin{array}{l}16.5 \\
16.0 \\
16.5 \\
17.0 \\
17.5\end{array}$ & $\begin{array}{l}17.5 \\
17.5 \\
17.5 \\
17.5 \\
17.5\end{array}$ & $\begin{array}{l}17.0 \\
17.0 \\
17.5 \\
17.0 \\
17.5\end{array}$ & $\begin{array}{l}17.0 \\
17.5 \\
17.5 \\
17.5 \\
17.5\end{array}$ & $\begin{array}{l}18.5 \\
18.5 \\
18.5 \\
22.0 \\
19.5\end{array}$ & $\begin{array}{l}18.0 \\
18.0 \\
18.0 \\
18.0 \\
18.5\end{array}$ & $\begin{array}{l}18.5 \\
18.0 \\
18.0 \\
19.5 \\
19.0\end{array}$ & $\begin{array}{l}19.5 \\
19.5 \\
20.5 \\
21.5 \\
-\end{array}$ & $\begin{array}{l}18.5 \\
18.5 \\
18.5 \\
19.0 \\
-\end{array}$ & $\begin{array}{r}19.0 \\
19.0 \\
19.5 \\
20.0\end{array}$ \\
\hline $\begin{array}{l}16 \\
17 \\
18 \\
19 \\
20\end{array}$ & $\begin{array}{l}20.0 \\
20.0 \\
20.5 \\
18.5 \\
17.0\end{array}$ & $\begin{array}{l}17.5 \\
17.5 \\
17.5 \\
16.0 \\
16.0\end{array}$ & $\begin{array}{l}18.0 \\
18.0 \\
18.0 \\
17.5 \\
16.5\end{array}$ & $\begin{array}{l}18.0 \\
17.5 \\
17.5 \\
17.5 \\
17.5\end{array}$ & $\begin{array}{l}17.5 \\
17.0 \\
17.0 \\
17.5 \\
17.0\end{array}$ & $\begin{array}{l}17.5 \\
17.5 \\
17.5 \\
17.5 \\
17.5\end{array}$ & $\begin{array}{l}19.0 \\
20.5 \\
19.5 \\
19.0 \\
19.0\end{array}$ & $\begin{array}{l}18.5 \\
18.0 \\
19.0 \\
18.5 \\
18.5\end{array}$ & $\begin{array}{l}18.5 \\
19.0 \\
19.0 \\
18.5 \\
19.0\end{array}$ & $\begin{array}{l}19.5 \\
22.0 \\
21.5 \\
20.0 \\
19.0\end{array}$ & $\begin{array}{l}19.0 \\
19.0 \\
19.5 \\
18.5 \\
18.0\end{array}$ & $\begin{array}{l}19.0 \\
20.0 \\
20.0 \\
19.0 \\
18.5\end{array}$ \\
\hline $\begin{array}{l}21 \\
22 \\
23 \\
24 \\
25\end{array}$ & $\begin{array}{l}16.5 \\
16.5 \\
17.0 \\
16.5 \\
16.5\end{array}$ & $\begin{array}{l}16.0 \\
16.0 \\
16.0 \\
16.0 \\
16.0\end{array}$ & $\begin{array}{l}16.5 \\
16.0 \\
16.5 \\
16.0 \\
16.0\end{array}$ & $\begin{array}{l}17.5 \\
18.0 \\
19.0 \\
19.5 \\
18.0\end{array}$ & $\begin{array}{l}17.5 \\
16.5 \\
17.5 \\
17.5 \\
17.5\end{array}$ & $\begin{array}{l}17.5 \\
17.5 \\
18.0 \\
18.0 \\
18.0\end{array}$ & $\begin{array}{l}20.5 \\
19.5 \\
20.5 \\
19.5 \\
19.5\end{array}$ & $\begin{array}{l}18.0 \\
18.5 \\
18.5 \\
18.5 \\
18.5\end{array}$ & $\begin{array}{l}19.0 \\
19.0 \\
19.0 \\
19.0 \\
19.0\end{array}$ & $\begin{array}{l}19.0 \\
18.0 \\
18.0 \\
18.0 \\
18.0\end{array}$ & $\begin{array}{l}18.0 \\
16.0 \\
15.5 \\
16.0 \\
16.5\end{array}$ & $\begin{array}{l}18.5 \\
17.5 \\
17.0 \\
17.0 \\
17.5\end{array}$ \\
\hline $\begin{array}{l}26 \\
27 \\
28 \\
29 \\
30 \\
31\end{array}$ & $\begin{array}{l}16.0 \\
16.0 \\
16.5 \\
16.5 \\
16.5 \\
-\end{array}$ & $\begin{array}{l}16.0 \\
15.5 \\
16.0 \\
16.0 \\
16.0 \\
-\end{array}$ & $\begin{array}{l}16.0 \\
16.0 \\
16.0 \\
16.0 \\
16.0\end{array}$ & $\begin{array}{l}17.5 \\
18.0 \\
18.5 \\
18.0 \\
18.0 \\
18.0\end{array}$ & $\begin{array}{l}17.0 \\
17.5 \\
17.5 \\
17.5 \\
17.5 \\
17.5\end{array}$ & $\begin{array}{l}17.5 \\
17.5 \\
17.5 \\
17.5 \\
17.5 \\
17.5\end{array}$ & $\begin{array}{l}19.5 \\
20.5 \\
20.5 \\
21.5 \\
21.5 \\
22.0\end{array}$ & $\begin{array}{l}18.5 \\
18.5 \\
19.0 \\
19.0 \\
19.5 \\
19.5\end{array}$ & $\begin{array}{l}19.0 \\
19.0 \\
19.5 \\
20.0 \\
20.5 \\
20.5\end{array}$ & $\begin{array}{l}18.0 \\
19.0 \\
18.5 \\
19.0 \\
20.5 \\
-\end{array}$ & $\begin{array}{l}17.0 \\
17.5 \\
18.0 \\
18.0 \\
18.0 \\
-\end{array}$ & $\begin{array}{l}18.0 \\
18.0 \\
18.5 \\
18.5 \\
19.0 \\
-\end{array}$ \\
\hline $10 \mathrm{NTH}$ & 20.5 & 14.5 & 16.3 & 19.5 & 16.0 & 17.2 & 22.0 & 17.5 & 18.7 & -- & $\cdots$ & $-\cdots$ \\
\hline
\end{tabular}




\section{CANADIAN RIVER MEAR WHITEFIEILD, OKIABOMA}

Iocarrow.--Lat $35^{\circ} 15^{\prime} 50^{\prime \prime}$, long $95^{\circ} 14^{\prime} 21^{\prime \prime}$, in SE1/4SE1/4, sec.12, T.9 N., R. 19 E., Haskell County, on left downstream ban at end of bridge, on State Highway $2,0.8 \mathrm{mi}$ north of whitefield, $5.5 \mathrm{mi}$ upstream from Taleka (Snake) Creek, $8.2 \mathrm{~m}$ downstream from Eufaula Dam, and at mile 18.8 .

DRATIAE AREA. - -47,576 $\mathrm{mi}^{2}$, of which $9,700 \mathrm{mi}^{2}$ is probably noncontributing.

AVERAGE DISchares. --25 years (water years 1939-63), 6,005 $\mathrm{ft}^{3} / \mathrm{s} ; 28$ years (water years 1968-95), 6, 902 ft ${ }^{3} / \mathrm{s}$.

Exurpars. - July 1938 to current year: Maximum discharge 281, $000 \mathrm{ft}^{3} / \mathrm{s}$ May 10, 1943; minimum da1ly $0.4 \mathrm{ft}^{3} / \mathrm{s}$ Oct. 8, 1956 REarks.--Records fair. Prior to February 1964, occasional slight regulation by Conchas Iake in New Mexico and excep for $54 \mathrm{mi}^{2}$ of intervening area, completely regulated thereafter by Eufaula Lake. Satellite telemeter at station.

Monthly and yearly discharge

\begin{tabular}{|c|c|c|c|c|c|}
\hline Month & $\begin{array}{l}\text { Totalal } \\
\left(\mathrm{ft}^{3} / \mathrm{s}\right)\end{array}$ & $\begin{array}{l}\text { Maximum } \\
\text { daily } \\
\left(\mathrm{ft}^{\frac{3}{3}} / \mathrm{s}\right)\end{array}$ & $\begin{array}{l}\text { Minimum } \\
\mathrm{dail}^{3} \mathrm{y} \\
\left(\mathrm{ft}^{3} / \mathrm{s}\right)\end{array}$ & $\begin{array}{c}\text { Mean } \\
\left(\mathrm{ft}^{3} / \mathrm{s}\right)\end{array}$ & $\begin{array}{c}\text { Runoff } \\
\text { (acre-feet) }\end{array}$ \\
\hline October & 18,371 & 3,090 & 59 & 593 & 36,440 \\
\hline November & 222,288 & 15,600 & 118 & 7,410 & 440,900 \\
\hline December & 292,010 & 18,600 & 2,260 & 9,420 & 579,200 \\
\hline January & 148,207 & 11,100 & 168 & 4,781 & 294,000 \\
\hline February & 158,896 & 13,200 & 106 & 5,675 & 315,200 \\
\hline March & 363,290 & 21,100 & 2,650 & 11,720 & 720,600 \\
\hline April & 337,860 & 21,300 & 1,970 & 11,260 & 670,100 \\
\hline May & 823,950 & 46,000 & 3,430 & 26,580 & $1,634,000$ \\
\hline June & 898,330 & 44,500 & 1,360 & 29,940 & $1,782,000$ \\
\hline July & 429,920 & 24,700 & 5,080 & 13,870 & 852,700 \\
\hline August & 181,150 & 11,700 & 1,560 & 5,844 & 359,300 \\
\hline September & 72,711 & 6,520 & 397 & 2,424 & 144,200 \\
\hline Water year 1995 & $3,946,983$ & 46,000 & 59 & 10,810 & $7,829,000$ \\
\hline
\end{tabular}




\section{POTEAU RIVER SUBBASIN}

\section{POTRAO RIVER AT CAOTHRON, ARKANSAS}

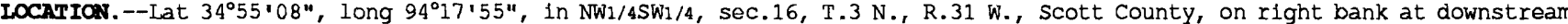
side of highway bridge at Cauthron, $2.9 \mathrm{mi}$ downstream from cross Creek, $7.8 \mathrm{mi}$ downstream from Jones Creek, and at mile 109.0.

DRAINAGE AREA. $--203 \mathrm{mi}^{2}$.

AVarace DIschares.--Prior to regulation, 35 years (water years 1940-74), 218 ft 3 /s; 21 years (water years 1975-95) $249 \mathrm{ft}^{3} / \mathrm{s}$.

EXIMRMES.-February 1939 to current year: Maximum discharge 32,200 ft ${ }^{3} / \mathrm{s}$ May 20, 1960 ; no flow at times in most years. REMaRks.--Records good. As of september 1974 , flow from $92.2 \mathrm{mi}^{2}$ above this station is controlled by 16 floodwaterdetention reservoirs with a total combined capacity of 39,082 acre-ft below the flood spillway crests, of which 33,524 acre-ft is flood-detention capacity, 2,100 acre-ft is water-supply storage, and 3,458 acre-ft is sediment-storage capac1ty. Satellite telemeter at station.

Monthly and yearly discharge

\begin{tabular}{|c|c|c|c|c|c|}
\hline Month & $\begin{array}{l}\text { Total } \\
\left(\mathrm{ft}^{3} / \mathrm{s}\right)\end{array}$ & $\begin{array}{c}\text { Maximum } \\
\text { daily } \\
\left(\mathrm{ft}^{3} / \mathrm{s}\right)\end{array}$ & $\begin{array}{l}\text { Minimum } \\
\text { daily } \\
\text { (ft } / \mathrm{s})\end{array}$ & $\underset{\left(\mathrm{ft}^{3} / \mathrm{s}\right)}{\operatorname{Mean}}$ & $\begin{array}{c}\text { Runoff } \\
\text { (acre-feet) }\end{array}$ \\
\hline October & 322 & 40 & 1.2 & 10.4 & 639 \\
\hline November & 33,363 & 8,180 & 12 & 1,112 & 66,180 \\
\hline December & 17,466 & 3,080 & 97 & 563 & 34,640 \\
\hline January & 21,953 & 4,690 & 114 & 708 & 43,540 \\
\hline February & 2,626 & 254 & 35 & 93.8 & 5,210 \\
\hline March & 4,566 & 737 & 33 & 147 & 9,060 \\
\hline April & 10,689 & 3,500 & 43 & 356 & 21,200 \\
\hline May & 23,079 & 8,540 & 38 & 744 & 45,780 \\
\hline June & $5,316.7$ & 733 & 7.3 & 177 & 10,550 \\
\hline July & 524.23 & 134 & .91 & 16.9 & 1,040 \\
\hline August & 63.18 & 5.2 & .26 & 2.04 & 125 \\
\hline September & 91.76 & 52 & .00 & 3.06 & 182 \\
\hline Water year 1995 & $120,059.87$ & 8,540 & .00 & 329 & 238,100 \\
\hline
\end{tabular}


PBRIOD OF RBCORD.--Water years 1945-61, 1975-79, December 1991 to current year.

RERRRs.--Specific conductance, pH, water temperature, dissolved oxygen, and alkalinity were determined in the field. WATER-QUALITY DATA, WATER YEAR OCTOBER 1994 TO SEPTEMBER 1995

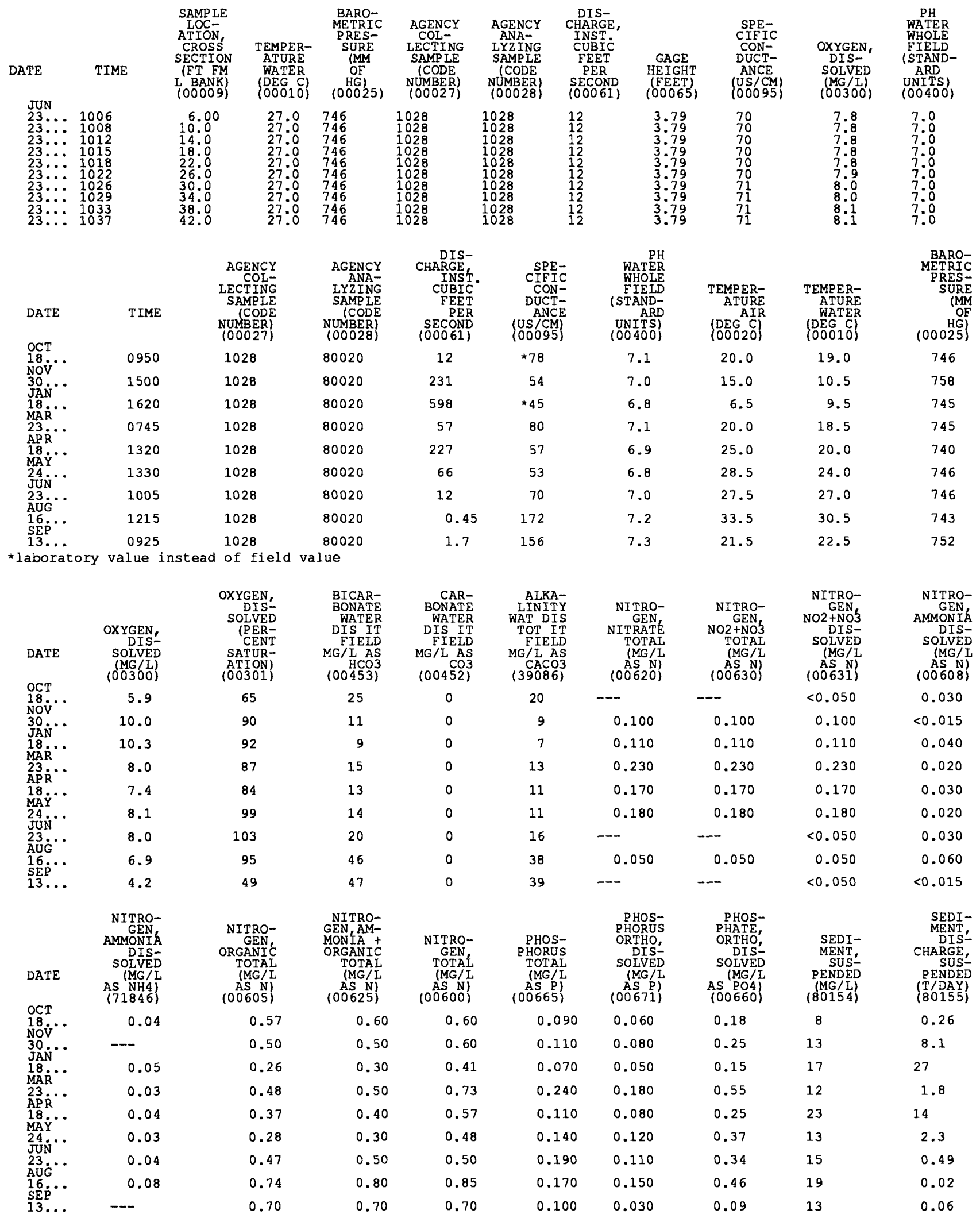


POTEAU RTVER SUBBASIN

07247000 POTEAO RIVER AT CAOTHRON, ARRANSAS--CONTINUED

WATER-QUALITY DATA, WATER YEAR OCTOBER 1994 TO SEPTEMBER 1995

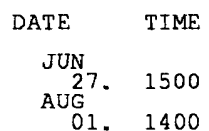

\begin{tabular}{|c|c|}
\hline $\begin{array}{l}\text { AGENCY } \\
\text { COL- } \\
\text { LECTING } \\
\text { SAMP LE } \\
\text { (CODE } \\
\text { NUMBER) } \\
(000027)\end{array}$ & $\begin{array}{l}\text { AGENCY } \\
\text { ANA- } \\
\text { LYZING } \\
\text { SAMPLE } \\
\text { (CODE } \\
\text { NUMBER) } \\
(00028)\end{array}$ \\
\hline 80513 & 81213 \\
\hline $\begin{array}{l}80513 \\
\text { E. COLI } \\
\text { WATER } \\
\text { WHOLE } \\
\text { TOTAL } \\
\text { UREASE } \\
\text { (COLS.' } \\
100 \text { ML') }\end{array}$ & $\begin{array}{r}81213 \\
\text { STREP- } \\
\text { TOCOCCI } \\
\text { FECAL } \\
\text { KF AGAR } \\
\text { (COLS } \\
100 \mathrm{ML} \text { ) }\end{array}$ \\
\hline K37 & 120 \\
\hline & 16 \\
\hline
\end{tabular}
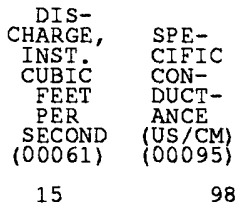

WATER

WHOLE

FIELD

ATAND

UNITS)
(00400)

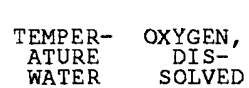
WATER $(00010)$ SOLVED (MG /L)
$(00300\}$ OXYGEN, DIS- FORM SOLVED FECAf, PERCENT
SATUR-MF
(COLS. ATION) $100 \mathrm{ML}$ 1598

$6.8 \quad 26.0$

6.9

8.929 .5

9.7

5.1

152

HARDNESS

CALCIUM MAGN- SODIUM, TOTAI

AS

(MG/L

SIUM, SODIUM,

SOLVED SOLVED

(MG/L (MG/L

AS MG) AS NA)
$(00925) \quad(00930)$

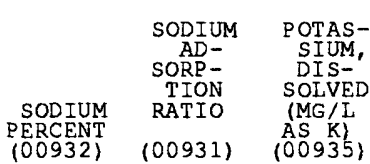

$\begin{array}{llll}17 & 2.7 & 2.5 & 14 \\ 23 & 3.4 & 3.6 & 24\end{array}$

59

1

2.9

61

8.2

SULFATE

CHLO-

SOLIDS

NITRO- NITRO-

SOLVED

DIS-'

AT 180

NITRATE NITRITE

NITRO-

NITRO- NITROGEN, NO2+NO3 AMMONIA DIS- SOLVED SOLVED TOTAL (MG/L SOLVED (MG/L SOLVED (MG/L MG/I (MG / L TM / (MG/L (MG/L AS SO4) AS CL) $(70300)$ AS N)
(00618)

$\begin{array}{cc}7.5 & 6.6 \\ 14 & 24\end{array}$

0.220 (00613) AS N) AS N) AS N

106

0.920

0.010

0.230

0.230

0.020

0.940

0.940

0.020

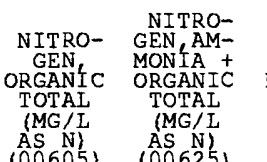

PHOS-
PHOS-

PHOS- PHORUS

HORUS

PHOS-

PHORUS

SOLVED

SOLVED

(MG/L (MG/L

AS P)
$(00666)$

AS P)

(0066)

0.44

0.45

0.190

0.140

0.130

SEDI-
MENT-
SUS-
PENDED

SEDI- SED.

MENT, SUSP.

CHARGE, DIAM.

SUS- FINER

TIDAY $.062 \mathrm{MM}$

$0.98 \quad 1.0 \quad 0.560$

0.400

0.430

1.4

92 


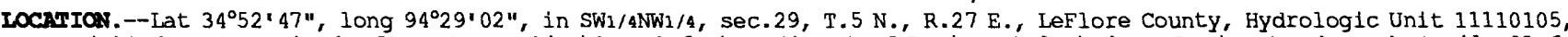
on right downstream bank of county road bridge, $0.6 \mathrm{mi}$ northwest of Loving, $1.0 \mathrm{mi}$ above Loving Creek, and at mile 93.6 . DRADMAS AREA. $--269 \mathrm{mt}^{2}$.

PERIOD OF RECORD. --Water years 1945-61, 1975-79, December 1991 to current year.

Ragrks.--Specific conductance, pH, water temperature, dissolved oxygen, and alkalinity were determined in the fleld. WATER YEAR OCTOBER 1993 TO SEPTEMBER 1994

\begin{tabular}{|c|c|c|c|c|c|c|c|c|c|c|c|}
\hline DATE & TIME & $\begin{array}{l}\text { SAMP LE } \\
\text { LOC- } \\
\text { ATION, } \\
\text { CROSS } \\
\text { SECTION } \\
\text { (FT FM } \\
\text { L BANK) } \\
(00009)\end{array}$ & $\begin{array}{c}\text { TEMPER- } \\
\text { ATURE } \\
\text { WATER } \\
\text { (DEG C) } \\
(00010)\end{array}$ & $\begin{array}{l}\text { BARO- } \\
\text { METRIC } \\
\text { PRES- } \\
\text { SURE } \\
\text { (MM } \\
\text { OF } \\
\text { HG) } \\
(00025)\end{array}$ & $\begin{array}{c}\text { AGENCY } \\
\text { COL- } \\
\text { LECTING } \\
\text { SAMPLE } \\
\text { (CODE } \\
\text { NUMBER) } \\
(00027)\end{array}$ & $\begin{array}{c}\text { AGENCY } \\
\text { ANA- } \\
\text { LYZING } \\
\text { SAMPLE } \\
\text { (CODE } \\
\text { NUMBER) } \\
(00028)\end{array}$ & $\begin{array}{c}\text { DIS- } \\
\text { CHARGE, } \\
\text { INST: } \\
\text { CUBIC } \\
\text { FEET } \\
\text { PER } \\
\text { SECOND } \\
(00061)\end{array}$ & $\begin{array}{c}\text { GAGE } \\
\text { HEIGHT } \\
(\text { EEET) } \\
(00065)\end{array}$ & $\begin{array}{c}\text { SPE- } \\
\text { CIEIC } \\
\text { CON- } \\
\text { DUCT- } \\
\text { ANCE } \\
(U S / C M) \\
(00095\rangle\end{array}$ & $\begin{array}{c}\text { OXYGEN, } \\
\text { DIS- } \\
\text { SOLVED } \\
\text { (MG/L) } \\
(00300)\end{array}$ & $\begin{array}{c}\text { PH } \\
\text { WATER } \\
\text { WHOLE } \\
\text { FIELD } \\
\text { (STAND } \\
\text { ARD } \\
\text { UNITS) } \\
(00400\end{array}$ \\
\hline $\begin{array}{l}\text { JUN } \\
23 \ldots \\
23 \ldots \\
23 \ldots \\
23 . \ldots \\
23 . \ldots \\
23 . \ldots \\
23 . \ldots \\
23 . \ldots \\
23 . \ldots \\
23 . \ldots \\
23 . \ldots\end{array}$ & $\begin{array}{l}0855 \\
0858 \\
0903 \\
0906 \\
0910 \\
0913 \\
0916 \\
0920 \\
0923 \\
0927 \\
0930\end{array}$ & $\begin{array}{l}10.0 \\
15: 0 \\
20.0 \\
25: 0 \\
50: 0 \\
55: 0 \\
60.0 \\
65: 0 \\
70: 0 \\
75: 0 \\
80.0\end{array}$ & $\begin{array}{l}26.0 \\
26.0 \\
26.0 \\
26.0 \\
26.0 \\
26.0 \\
26.0 \\
26.0 \\
26.0 \\
26.0 \\
26.0\end{array}$ & $\begin{array}{l}745 \\
745 \\
745 \\
745 \\
745 \\
745 \\
745 \\
745 \\
745 \\
745 \\
745\end{array}$ & $\begin{array}{l}1028 \\
1028 \\
1028 \\
1028 \\
1028 \\
1028 \\
1028 \\
1028 \\
1028 \\
1028 \\
1028\end{array}$ & $\begin{array}{l}1028 \\
1028 \\
1028 \\
1028 \\
1028 \\
1028 \\
1028 \\
1028 \\
1028 \\
1028 \\
1028\end{array}$ & $\begin{array}{l}27 \\
27 \\
27 \\
27 \\
27 \\
27 \\
27 \\
27 \\
27 \\
27 \\
27\end{array}$ & $\begin{array}{l}9.00 \\
9.00 \\
9.00 \\
9.00 \\
9.00 \\
9.00 \\
9.00 \\
9.00 \\
9.00 \\
9.00 \\
9.00\end{array}$ & $\begin{array}{l}73 \\
73 \\
73 \\
73 \\
73 \\
73 \\
73 \\
73 \\
73 \\
73 \\
73\end{array}$ & $\begin{array}{l}5.6 \\
5.6 \\
5.8 \\
6.0 \\
5.4 \\
6.1 \\
5.8 \\
5.8 \\
6.0 \\
5.9 \\
6.0\end{array}$ & $\begin{array}{l}6.8 \\
6.8 \\
6.8 \\
6.8 \\
6.8 \\
6.8 \\
6.8 \\
6.8 \\
6.8 \\
6.8 \\
6.8\end{array}$ \\
\hline
\end{tabular}

\begin{tabular}{|c|c|c|c|c|c|c|c|c|c|}
\hline DATE & TIME & $\begin{array}{r}\text { AGENCY } \\
\text { COL- } \\
\text { LECTING } \\
\text { SAMPLIE } \\
\text { (CODE } \\
\text { NUMBER) } \\
(00027)\end{array}$ & $\begin{array}{r}\text { AGENCY } \\
\text { ANA- } \\
\text { LYZING } \\
\text { SAMPIE } \\
\text { (CODE } \\
\text { NUMBER) } \\
(00028)\end{array}$ & $\begin{array}{c}\text { DIS- } \\
\text { CHARGE, } \\
\text { INST. } \\
\text { CUBIC } \\
\text { FEET } \\
\text { PER } \\
\text { SECOND } \\
(00061)\end{array}$ & $\begin{array}{r}\text { SPE- } \\
\text { CIFIC } \\
\text { CON- } \\
\text { DUCT- } \\
\text { ANCE } \\
(\text { US } / C M) \\
00095)\end{array}$ & $\begin{array}{r}\text { PH } \\
\text { WATER } \\
\text { WHOLE } \\
\text { FIEID } \\
\text { (STAND- } \\
\text { ARD } \\
\text { UNITS) } \\
\text { (O040O) }\end{array}$ & $\begin{array}{r}\text { TEMPER- } \\
\text { ATURE } \\
\text { AIR } \\
\left(\begin{array}{c}\text { DEG C } \\
00020\end{array}\right)\end{array}$ & $\begin{array}{c}\text { TEMPER- } \\
\text { ATURE } \\
\text { WATER } \\
(\text { DEG C ) } \\
(00010)\end{array}$ & $\begin{array}{r}\text { BARO- } \\
\text { METRIC } \\
\text { PRES } \\
\text { SURE } \\
\text { (MM } \\
\text { OF } \\
\text { HG) } \\
(0025)\end{array}$ \\
\hline $\begin{array}{l}\text { OCT } \\
18 . . .\end{array}$ & 1155 & 1028 & 80020 & 0.99 & $\star 82$ & 7.0 & 21.5 & 20.0 & 746 \\
\hline $01 \ldots$ & 0955 & 1028 & 80020 & 255 & 58 & 6.9 & 9.5 & 9.5 & 763 \\
\hline $19 \ldots$ & 1015 & 1028 & 80020 & 2380 & 51 & 6.9 & 3.0 & 7.5 & 750 \\
\hline $23 \ldots$ & 0900 & 1028 & 80020 & 58 & 83 & 7.4 & 21.0 & 19.5 & 747 \\
\hline $18 \ldots$ & 1730 & 1028 & 80020 & 235 & 62 & 6.8 & 27.0 & 20.0 & 745 \\
\hline $25 \ldots$ & 1140 & 1028 & 80020 & 84 & 58 & 6.7 & 23.0 & 24.0 & 752 \\
\hline $23 \ldots$ & 0850 & 1028 & 80020 & 27 & 73 & 6.8 & 25.5 & 26.0 & 745 \\
\hline $18 \ldots$ & 0805 & 1028 & 80020 & 4.8 & 141 & 7.1 & 24.0 & 27.5 & 748 \\
\hline $13 \ldots$ & 0805 & 1028 & 80020 & 4.8 & 140 & 7.5 & 20.0 & 21.5 & 753 \\
\hline
\end{tabular}

*laboratory value instead of field value

\begin{tabular}{|c|c|c|c|}
\hline DATE & $\begin{array}{c}\text { OXYGEN, } \\
\text { DIS } \\
\text { SOLVED } \\
(M G / I) \\
(00300)\end{array}$ & $\begin{array}{r}\text { DIS- } \\
\text { SOLVED } \\
\text { (PER- } \\
\text { CENT } \\
\text { SATUR- } \\
\text { ATION) } \\
\text { (00301) }\end{array}$ & $\begin{array}{r}\text { BONATE } \\
\text { WATER } \\
\text { DIS IT } \\
\text { FIELD } \\
\text { MG/L AS } \\
\text { HCO } \\
(00453)\end{array}$ \\
\hline $\begin{array}{l}\text { OCT } \\
18 . . .\end{array}$ & 4.9 & 55 & 29 \\
\hline & 11.2 & 98 & 13 \\
\hline $19 \ldots$ & 10.8 & 91 & 10 \\
\hline $23 \ldots$ & 8.0 & 89 & 21 \\
\hline 18. & 7.0 & 79 & 15 \\
\hline $25 \ldots$ & 6.9 & 83 & 15 \\
\hline $23 \ldots$ & 6.0 & 76 & 18 \\
\hline $18 \ldots$ & 4.3 & 55 & 35 \\
\hline $13 \ldots$ & 4.6 & 53 & 44 \\
\hline
\end{tabular}

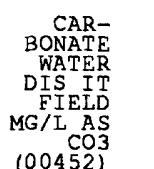

(00452)

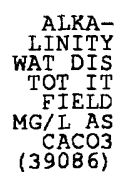

24

11

8

17

12

12

14

29

36

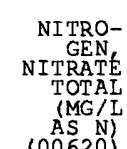

AS N)
$(00620)$

0.070

0.100

0.120

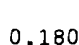

0.180

0.130

0.110

0.070

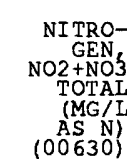

0.070

0.100

0.120

0.180

0.100

0.130

0.110

0.070

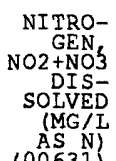

(00631)

0.070

0.100

0.120

$<0.050$

0.180

0.100

0.130

0.110

0.070
NITRO-

AMMONIÁ

SOLVED

(MG/L

AS N
$(00608)$

0.090

$<0.015$

0.040

0.020

0.030

0.030

0.050

0.060

0.020 
POTEAU RIVER SUBBASIN

07247015 POTEAD RIVER AT LOVING, OKLAHOMA--CONTINOED

\begin{tabular}{|c|c|c|c|c|c|c|c|c|c|}
\hline DATE & $\begin{array}{c}\text { NITRO- } \\
\text { GEN, } \\
\text { AMMONIA } \\
\text { DIS - } \\
\text { SOLVED } \\
\text { (MG/L } \\
\text { AS NH4) } \\
(71846)\end{array}$ & $\begin{array}{c}\text { NITRO- } \\
\text { GEN } \\
\text { ORGANIC } \\
\text { TOTAL } \\
\text { (MG } \\
\text { AS N } \\
(00605)\end{array}$ & $\begin{array}{c}\text { NITRO- } \\
\text { GEN AM- } \\
\text { MONIA + } \\
\text { ORGANIC } \\
\text { TOTAL } \\
\text { (MG/I } \\
\text { AS N) } \\
(00625)\end{array}$ & $\begin{array}{c}\text { NITRO- } \\
\text { GEN } \\
\text { TOTAf } \\
(M G / I \\
\text { AS N) } \\
(00600)\end{array}$ & $\begin{array}{c}\text { PHOS- } \\
\text { PHORUS } \\
\text { TOTAL } \\
(\mathrm{MG} / \mathrm{L} \\
\text { AS } \mathrm{P}) \\
(00665)\end{array}$ & $\begin{array}{c}\text { PHOS- } \\
\text { PHORUS } \\
\text { ORTHO, } \\
\text { DIS - } \\
\text { SOLVED } \\
\text { (MG/L } \\
\text { AS P }) \\
(00671)\end{array}$ & $\begin{array}{r}\text { PHOS- } \\
\text { PHATE, } \\
\text { ORTHO' } \\
\text { DIS' } \\
\text { SOIVED } \\
\text { (MG/I } \\
\text { AS POL) } \\
(00660)\end{array}$ & $\begin{array}{r}\text { SEDI- } \\
\text { MENT, } \\
\text { SUS- } \\
\text { PENDED } \\
\text { (MG/L) } \\
(80154)\end{array}$ & $\begin{array}{r}\text { SEDI- } \\
\text { MENT, } \\
\text { DIS- } \\
\text { CHARGE' } \\
\text { SUS- } \\
\text { PENDED } \\
\text { (T/DAY) } \\
(80155 \text { ) }\end{array}$ \\
\hline $\mathrm{OCT}$ & 0.12 & 0.61 & 0.70 & 0.77 & 0.050 & 0.030 & 0.09 & 7 & 0.02 \\
\hline $\begin{array}{l}\text { DEC } \\
01 . . .\end{array}$ & --- & 0.40 & 0.40 & 0.50 & 0.100 & 0.060 & 0.18 & 13 & 9.0 \\
\hline $19 .$. & 0.05 & 0.36 & 0.40 & 0.52 & 0.080 & 0.050 & 0.15 & 70 & 450 \\
\hline $23 \ldots$ & 0.03 & 0.38 & 0.40 & 0.40 & 0.120 & 0.080 & 0.25 & 15 & 2.3 \\
\hline $18 . \cdots$ & 0.04 & 0.37 & 0.40 & 0.58 & 0.100 & 0.060 & 0.18 & 19 & 12 \\
\hline $25 \ldots$ & 0.04 & 0.17 & 0.20 & 0.30 & 0.040 & 0.030 & 0.09 & 13 & 2.9 \\
\hline $23 . \cdots$ & 0.06 & 0.55 & 0.60 & 0.73 & 0.110 & 0.050 & 0.15 & 22 & 1.6 \\
\hline $18 .$. & 0.08 & 0.44 & 0.50 & 0.61 & 0.070 & 0.070 & 0.21 & 19 & 0.25 \\
\hline $13 \ldots$ & 0.03 & 0.58 & 0.60 & 0.67 & 0.050 & 0.010 & 0.03 & 9 & 0.12 \\
\hline
\end{tabular}




\section{POTEAU RTVER SUBBASIN}

07247250 BIACK FORK BELON BIG CREEK MEAR PAGE, OKLAHOMA

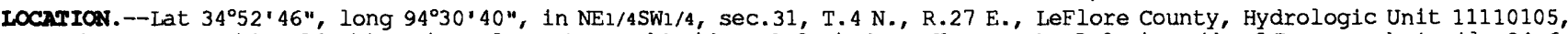
on downstream side of bridge pier of county road bridge, $2.2 \mathrm{mi}$ above Haw Creek, $5.0 \mathrm{mi}$ north of Page, and at mile 24.6 . DRADAGE AREA. $-74.4 \mathrm{mi}^{2}$.

PERIOD OF RECORD.--December 1991 to current year.

REarks.--Specific conductance, $\mathrm{pH}$, water temperature, dissolved oxygen, and alkalinity were determined in the fleld. WATER-QUALITY DATA, WATER YEAR OCTOBER 1994 TO SEPTEMBER 1995

\begin{tabular}{|c|c|c|c|c|c|c|c|c|c|}
\hline DATE & TIME & $\begin{array}{l}\text { SAMP LE } \\
\text { LOC- } \\
\text { ATION, } \\
\text { CROSS } \\
\text { SECTION } \\
\text { (FT FM } \\
\text { L BANK) } \\
(00009)\end{array}$ & $\begin{array}{l}\text { BARO- } \\
\text { METRIC } \\
\text { PRES- } \\
\text { SURE } \\
\text { (MM } \\
\text { OF } \\
\text { HG) } \\
(00025)\end{array}$ & $\begin{array}{c}\text { AGENCY } \\
\text { COL- } \\
\text { LECTING } \\
\text { SAMPIE } \\
\text { (CODE } \\
\text { NUMBER) } \\
(00027)\end{array}$ & $\begin{array}{l}\text { AGENCY } \\
\text { ANA- } \\
\text { LYZING } \\
\text { SAMPLE } \\
\text { (CODE } \\
\text { NUMBER } \\
(00028)\end{array}$ & $\begin{array}{l}\text { DIS- } \\
\text { CHARGE, } \\
\text { INST } \\
\text { CUBIC } \\
\text { FEET } \\
\text { PER } \\
\text { SECOND } \\
(00061)\end{array}$ & $\begin{array}{c}\text { SPE- } \\
\text { CIFIC } \\
\text { CON- } \\
\text { DUCT- } \\
\text { ANCE } \\
(\text { US } / C M) \\
(00095)\end{array}$ & $\begin{array}{l}\text { OXYGEN, } \\
\text { DIS- } \\
\text { SOLVED } \\
(M G / L) \\
(00300)\end{array}$ & $\begin{array}{c}\text { PH } \\
\text { WATER } \\
\text { WHOLE } \\
\text { FIELD } \\
\text { (STAND- } \\
\text { ARD } \\
\text { UNITS) } \\
\text { (00400) }\end{array}$ \\
\hline $\begin{array}{l}23 \ldots \\
23 \ldots \\
23 \ldots \\
23 \ldots \\
23 \ldots \\
23 \ldots \\
23 \ldots \\
23 \ldots \\
23 \ldots\end{array}$ & $\begin{array}{l}0733 \\
0736 \\
0739 \\
0742 \\
0745 \\
0748 \\
0753 \\
0755 \\
0758\end{array}$ & $\begin{array}{r}6.00 \\
10.0 \\
14.0 \\
18.0 \\
22.0 \\
26.0 \\
30.0 \\
34.0 \\
38.0\end{array}$ & $\begin{array}{l}743 \\
743 \\
743 \\
743 \\
743 \\
743 \\
743 \\
743 \\
743\end{array}$ & $\begin{array}{l}1028 \\
1028 \\
1028 \\
1028 \\
1028 \\
1028 \\
1028 \\
1028 \\
1028\end{array}$ & $\begin{array}{l}1028 \\
1028 \\
1028 \\
1028 \\
1028 \\
1028 \\
1028 \\
1028 \\
1028\end{array}$ & $\begin{array}{l}4.03 \\
4.03 \\
4.03 \\
4.03 \\
4.03 \\
4.03 \\
4.03 \\
4.03 \\
4.03\end{array}$ & $\begin{array}{l}37 \\
37 \\
37 \\
37 \\
37 \\
37 \\
37 \\
37 \\
37\end{array}$ & $\begin{array}{l}6.2 \\
6: 1 \\
6.4 \\
6.4 \\
6.0 \\
6: 2 \\
6.2 \\
6.2 \\
6.2\end{array}$ & $\begin{array}{l}6.8 \\
6.8 \\
6.8 \\
6.8 \\
6.8 \\
6.8 \\
6.8 \\
6.8 \\
6.8\end{array}$ \\
\hline DATE & TIME & $\begin{array}{r}\text { AGENCY } \\
\text { COL- } \\
\text { LECTING } \\
\text { SAMPLE } \\
\text { (CODE } \\
\text { NUMBER) } \\
(00027)\end{array}$ & $\begin{array}{c}\text { AGENCY } \\
\text { ANA- } \\
\text { LYZING } \\
\text { SAMPIE } \\
\text { (CODE } \\
\text { NUMBER) } \\
(00028)\end{array}$ & $\begin{array}{c}\text { DIS- } \\
\text { CHARGE, } \\
\text { INST. } \\
\text { CUBIC } \\
\text { FEET } \\
\text { PER } \\
\text { SECOND } \\
(00061)\end{array}$ & $\begin{array}{r}\text { SPE- } \\
\text { CIFIC } \\
\text { CON- } \\
\text { DUCT- } \\
\text { ANCE } \\
\left(\begin{array}{c}\text { US } / C M) \\
(00095)\end{array}\right.\end{array}$ & $\begin{array}{r}\text { PH } \\
\text { WATER } \\
\text { WHOLE } \\
\text { FIELD } \\
\text { (STAND- } \\
\text { ARD } \\
\text { UNITS) } \\
(00400 \text { ) }\end{array}$ & $\begin{array}{r}\text { TEMPER- } \\
\text { ATURE } \\
\text { AIR } \\
\left(\begin{array}{c}\text { DEG C) } \\
00020\end{array}\right)\end{array}$ & $\begin{array}{c}\text { TEMPER- } \\
\text { ATURE } \\
\text { WATER } \\
\left(\begin{array}{l}\text { DEG C } \\
00010\end{array}\right)\end{array}$ & $\begin{array}{r}\text { BARO- } \\
\text { METRIC } \\
\text { PRES- } \\
\text { SURE } \\
\text { (MM } \\
\text { OF } \\
\text { HG) } \\
(00025)\end{array}$ \\
\hline $\begin{array}{l}\text { OCT } \\
18 . . .\end{array}$ & 1400 & 1028 & 80020 & 3.7 & 46 & 7.0 & 24.5 & 20.5 & 740 \\
\hline $01 . .$. & 1100 & 1028 & 80020 & 104 & 30 & 6.7 & 15.5 & 9.0 & 757 \\
\hline $19 \ldots$ & 1130 & 1028 & 80020 & 882 & 25 & 6.5 & 7.5 & 7.0 & 750 \\
\hline $23 \ldots$ & 1005 & 1028 & 80020 & 59 & 29 & 6.8 & 24.0 & 20.0 & 740 \\
\hline 19. & 1040 & 1028 & 80020 & 151 & 28 & 6.9 & 18.5 & 17.0 & 745 \\
\hline $25 \ldots$ & 1235 & 1028 & 80020 & 37 & 32 & 6.6 & 25.0 & 23.5 & 746 \\
\hline $23 \ldots$ & 0725 & 1028 & 80020 & 8.7 & 38 & 6.8 & 21.0 & 25.0 & 743 \\
\hline DATE & $\begin{array}{c}\text { OXYGEN, } \\
\text { DIS- } \\
\text { SOLVED } \\
(M G / L) \\
(00300)\end{array}$ & $\begin{array}{r}\text { OXYGEN, } \\
\text { DIS- } \\
\text { SOLVED } \\
\text { (PER- } \\
\text { CENT } \\
\text { SATUR- } \\
\text { ATION) } \\
\text { (OO3OI) }\end{array}$ & $\begin{array}{c}\text { BICAR- } \\
\text { BONATE } \\
\text { WATER } \\
\text { DIS IT } \\
\text { FIELD } \\
\text { MG/L AS } \\
\text { HCO3 } \\
(00453)\end{array}$ & $\begin{array}{r}\text { CAR- } \\
\text { BONATE } \\
\text { WATER } \\
\text { DIS IT } \\
\text { FIELD } \\
\text { MG/L AS } \\
\text { CO3 } \\
(00452)\end{array}$ & $\begin{array}{r}\text { ALKA- } \\
\text { LINITY } \\
\text { WAT DIS } \\
\text { TOT IT } \\
\text { FIEID } \\
\text { MG/L AS } \\
\text { CACO3 } \\
(39086)\end{array}$ & $\begin{array}{c}\text { NITRO- } \\
\text { GEN } \\
\text { NITRATE } \\
\text { TOTAL } \\
\text { (MG/L } \\
\text { AS N) } \\
(00620)\end{array}$ & $\begin{array}{c}\text { NI TRO- } \\
\text { GEN } \\
\text { NO2+NO3 } \\
\text { TOTAL } \\
\text { (MG/I } \\
\text { AS N } \\
(00630)\end{array}$ & $\begin{array}{c}\text { NITRO- } \\
\text { GEN } \\
\text { NO2+NO3 } \\
\text { DIS- } \\
\text { SOLVED } \\
\text { (MG/L } \\
\text { AS N) } \\
(00631)\end{array}$ & $\begin{array}{r}\text { NITRO- } \\
\text { GEN } \\
\text { AMMONIA } \\
\text { DIS- } \\
\text { SOLVED } \\
\text { (MG/L } \\
\text { AS N) } \\
(00608)\end{array}$ \\
\hline $18 . .$. & 8.3 & 95 & 11 & 0 & 9 & 0.060 & 0.060 & 0.060 & 0.040 \\
\hline 01. & 10.8 & 94 & 5 & 0 & 4 & 0.090 & 0.090 & 0.090 & $<0.015$ \\
\hline $19{ }^{19}$ & 11.5 & 97 & 5 & 0 & 4 & 0.100 & 0.100 & 0.100 & $<0.015$ \\
\hline $23 \ldots$ & 7.8 & 89 & 7 & 0 & 6 & $-\cdots$ & $-\infty$ & $<0.050$ & $<0.015$ \\
\hline $19 \ldots$ & 8.7 & 92 & 7 & 0 & 5 & $-\cdots$ & $-\cdots$ & $<0.050$ & $<0.015$ \\
\hline 25. & 6.0 & 72 & 9 & 0 & 7 & 0.090 & 0.090 & 0.090 & 0.030 \\
\hline $23 \ldots$ & 6.4 & 80 & 12 & 0 & 10 & -- & $-\cdots$ & $<0.050$ & 0.060 \\
\hline DATE & $\begin{array}{c}\text { NITRO- } \\
\text { GEN } \\
\text { AMMONIA } \\
\text { DIS- } \\
\text { SOLVED } \\
\text { (MG/I } \\
\text { AS NH4) } \\
(71846)\end{array}$ & $\begin{array}{c}\text { NITRO- } \\
\text { GEN, } \\
\text { ORGANIC } \\
\text { TOTAL } \\
\text { (MG/L } \\
\text { AS N) } \\
(00605)\end{array}$ & $\begin{array}{c}\text { NITRO- } \\
\text { GEN AM- } \\
\text { MONIA + } \\
\text { ORGANIC } \\
\text { TOTAL } \\
\text { (MG/I } \\
\text { AS N) } \\
(00625)\end{array}$ & $\begin{array}{c}\text { NITRO- } \\
\text { GEN, } \\
\text { TOTAL } \\
\text { (MG/I } \\
\text { AS N) } \\
(00600)\end{array}$ & $\begin{array}{c}\text { PHOS- } \\
\text { PHORUS } \\
\text { TOTAL } \\
\text { (MG/L } \\
\text { AS P) } \\
(00665)\end{array}$ & $\begin{array}{c}\text { PHOS- } \\
\text { PHORUS } \\
\text { ORTHO, } \\
\text { DIS- } \\
\text { SOLVED } \\
\text { (MG/L } \\
\text { AS P) } \\
(00671)\end{array}$ & $\begin{array}{c}\text { PHOS- } \\
\text { PHATE; } \\
\text { ORTHO, } \\
\text { DIS- } \\
\text { SOLVED } \\
\text { (MG/L } \\
\text { AS PO4) } \\
(00660)\end{array}$ & $\begin{array}{c}\text { SEDI- } \\
\text { MENT, } \\
\text { SUS- } \\
\text { PENDED } \\
(\text { MG/L) } \\
(80154)\end{array}$ & $\begin{array}{r}\text { SEDI- } \\
\text { MENT, } \\
\text { DISS- } \\
\text { CHARGE, } \\
\text { SUS - } \\
\text { PENDED } \\
\text { (T/DAY) } \\
(80155)\end{array}$ \\
\hline $18 .$. & 0.05 & 0.46 & 0.50 & 0.56 & 0.020 & 0.010 & 0.03 & 6 & 0.06 \\
\hline 01. & -- & $\cdots$ & $<0.20$ & -- & 0.020 & $<0.010$ & -- & 6 & 1.7 \\
\hline $19 .$. & $\cdots$ & 0.20 & 0.20 & 0.30 & 0.020 & 0.020 & 0.06 & 13 & 31 \\
\hline $23 \ldots$ & -- & $\cdots$ & $<0.20$ & -- & 0.020 & $<0.010$ & -- & 4 & 0.64 \\
\hline 19. & --- & -- & $<0.20$ & -- & $<0.010$ & $<0.010$ & $-\cdots$ & 5 & 2.0 \\
\hline $25 \ldots$ & 0.04 & --- & $<0.20$ & --- & $<0.010$ & $<0.010$ & -- & 6 & 0.60 \\
\hline $23 \ldots$ & 0.08 & 0.34 & 0.40 & 0.40 & 0.030 & 0.020 & 0.06 & 9 & 0.21 \\
\hline
\end{tabular}




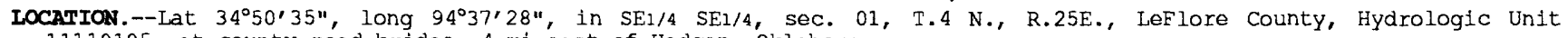
11110105 , at county róad bridge $.4 \mathrm{mi}$ east of Hodgen, Oklahoma.

DRATIAGE ARMA. $-179 \mathrm{mi}^{2}$.

PERIOD OF RECORD.--December 1991 to current year.

REMaRKs.--Specific conductance, pH, water temperature, dissolved oxygen, and alkalinity were determined in the field. WATER-QUALITY DATA, WATER YEAR OCTOBER 1994 TO SEPTEMBER 1995

\begin{tabular}{|c|c|c|c|c|c|c|c|c|c|c|}
\hline DATE & TIME & $\begin{array}{r}\text { TEMPER- } \\
\text { ATURE } \\
\text { WATER } \\
\{\text { DEG C } \\
00010\}\end{array}$ & $\begin{array}{r}\text { BARO- } \\
\text { METRIC } \\
\text { PRES- } \\
\text { SURE } \\
\text { (MM } \\
\text { OF } \\
\text { HG } \\
\{00025\}\end{array}$ & $\begin{array}{r}\text { AGENCY } \\
\text { COL- } \\
\text { LECTING } \\
\text { SAMPLE } \\
\text { (CODE } \\
\text { NUMBER) } \\
(00027)\end{array}$ & $\begin{array}{c}\text { AGENCY } \\
\text { ANA- } \\
\text { IYZING } \\
\text { SAMPLE } \\
\text { CODE } \\
\text { NUMBER } \\
(0002 B)\end{array}$ & $\begin{array}{c}\text { DIS- } \\
\text { CHARGE } \\
\text { INST. } \\
\text { CUBIC } \\
\text { FEET } \\
\text { PER } \\
\text { SECOND } \\
(00061)\end{array}$ & $\begin{array}{r}\text { GAGE } \\
\text { HEIGHT } \\
(\text { (EEET) } \\
(00065)\end{array}$ & $\begin{array}{r}\text { SPE- } \\
\text { CIFIC } \\
\text { CON- } \\
\text { DUCT- } \\
\text { ANCE } \\
(\text { US /CM }) \\
(00095)\end{array}$ & $\begin{array}{c}\text { OXYGEN, } \\
\text { DIS- } \\
\text { SOLVED } \\
(M G / L) \\
(00300)\end{array}$ & $\begin{array}{r}\text { WAT } \\
\text { WHO } \\
\text { FIE } \\
\text { ISTAN } \\
\text { A } \\
\text { UNIT } \\
10040\end{array}$ \\
\hline $\begin{array}{l}22 \ldots \\
22 \ldots \\
22 \ldots \\
22 \ldots \\
22 \ldots \\
22 \ldots \\
22 \ldots\end{array}$ & $\begin{array}{l}1335 \\
1338 \\
1341 \\
1344 \\
1347 \\
1350 \\
1354\end{array}$ & $\begin{array}{l}2 B \cdot 5 \\
2 B \cdot 5 \\
2 B \cdot 5 \\
2 B \cdot 5 \\
2 B \cdot 5 \\
2 B \cdot 5 \\
28 \cdot 5\end{array}$ & $\begin{array}{l}744 \\
744 \\
744 \\
744 \\
744 \\
744 \\
744\end{array}$ & $\begin{array}{l}1028 \\
1028 \\
1028 \\
1028 \\
1028 \\
1028 \\
1028\end{array}$ & $\begin{array}{l}1028 \\
1028 \\
1028 \\
1028 \\
1028 \\
1028 \\
1028\end{array}$ & $\begin{array}{l}19 \\
19 \\
19 \\
19 \\
19 \\
19 \\
19\end{array}$ & $\begin{array}{l}9.47 \\
9.47 \\
9.47 \\
9.47 \\
9.47 \\
9.47 \\
9.47\end{array}$ & $\begin{array}{l}42 \\
42 \\
42 \\
42 \\
42 \\
42 \\
42\end{array}$ & $\begin{array}{l}7.0 \\
7: 2 \\
7: 3 \\
7: 4 \\
7.4 \\
7.4 \\
7.4\end{array}$ & $\begin{array}{l}6.8 \\
6.8 \\
6.8 \\
6.8 \\
6.8 \\
6.8 \\
6.8\end{array}$ \\
\hline
\end{tabular}

\begin{tabular}{|c|c|c|c|c|c|c|c|c|c|}
\hline DATE & TIME & $\begin{array}{r}\text { AGENCY } \\
\text { COL- } \\
\text { LECTING } \\
\text { SAMPLE } \\
\text { (CODE } \\
\text { NUMBER) } \\
(00027)\end{array}$ & $\begin{array}{r}\text { AGENCY } \\
\text { ANA - } \\
\text { LYZINS } \\
\text { SAMPLE } \\
\text { (CODE } \\
\text { NUMBER) } \\
(0002 B)\end{array}$ & $\begin{array}{c}\text { DIS- } \\
\text { CHARGE } \\
\text { INST. } \\
\text { CUBIC } \\
\text { FEET } \\
\text { PER } \\
\text { SECOND } \\
(00061)\end{array}$ & $\begin{array}{r}\text { SPE- } \\
\text { CIFIC } \\
\text { CON- } \\
\text { DUCT- } \\
\text { ANCE } \\
(\text { US /CM }) \\
(00095)\end{array}$ & $\begin{array}{r}\text { PH } \\
\text { WATER } \\
\text { WHOLE } \\
\text { FIEID } \\
\text { (STAND- } \\
\text { ARD } \\
\text { UNITS) } \\
\text { (OO4OO) }\end{array}$ & $\begin{array}{r}\text { TEMPER- } \\
\text { ATURE } \\
\text { AIR } \\
(\text { DEG C } \\
\{00020\}\end{array}$ & $\begin{array}{r}\text { TEMPER- } \\
\text { ATURE } \\
\text { WATER } \\
\text { (DEG C) } \\
(00010)\end{array}$ & $\begin{array}{r}\text { BARO- } \\
\text { METRIC } \\
\text { PRES- } \\
\text { SURE } \\
\text { (MM } \\
\text { OF } \\
\text { HG) } \\
(00025\}\end{array}$ \\
\hline \multirow{9}{*}{ 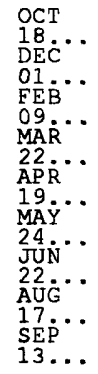 } & 1515 & 1028 & 80020 & 11 & $\star 46$ & 7.1 & 24.5 & 21.0 & 745 \\
\hline & 1230 & 1028 & 80020 & 221 & 38 & 6.8 & 11.0 & 11.0 & 760 \\
\hline & 1630 & 1028 & 80020 & 122 & 37 & 7.6 & 10.5 & 7.5 & 751 \\
\hline & 1210 & 1028 & 80020 & 102 & 37 & 7.3 & 29.0 & 20.5 & 738 \\
\hline & 1200 & 1028 & 80020 & 309 & $\star 35$ & 6.9 & 18.0 & 18.0 & 749 \\
\hline & 1450 & 1028 & 80020 & 55 & *39 & 7.0 & 26.5 & 25.5 & 747 \\
\hline & 1315 & 1028 & 80020 & 19 & 44 & 6.8 & 29.5 & 28.0 & 744 \\
\hline & 1220 & 1028 & 80020 & 0.40 & 57 & 6.8 & 35.5 & 31.5 & 745 \\
\hline & 1600 & 1028 & 80020 & 0.04 & 63 & 7.0 & 24.0 & 24.5 & 751 \\
\hline \multicolumn{10}{|c|}{ laboratory value instead of field value } \\
\hline DATE & $\begin{array}{r}\text { OXYGEN, } \\
\text { DIS' } \\
\text { SOIVED } \\
(\mathrm{MG} / \mathrm{L}) \\
(00300)\end{array}$ & $\begin{array}{c}\text { OXYGEN, } \\
\text { DIS- } \\
\text { SOIVED } \\
\text { (PER- } \\
\text { CENT } \\
\text { SATUR- } \\
\text { ATION) } \\
\text { (OO301) }\end{array}$ & $\begin{array}{c}\text { BICAR- } \\
\text { BONATE } \\
\text { WATER } \\
\text { DIS IT } \\
\text { FIEID } \\
\text { MG/L AS } \\
\text { HCO3 } \\
(00453)\end{array}$ & $\begin{array}{r}\text { CAR- } \\
\text { BONATE } \\
\text { WATER } \\
\text { DIS IT } \\
\text { FIEID } \\
\text { MG } / \text { A AS } \\
\text { COS } \\
(00452)\end{array}$ & $\begin{array}{r}\text { AIKA- } \\
\text { IINITY } \\
\text { WAT DIS } \\
\text { TOT IT } \\
\text { FIELD } \\
\text { MG/L AS } \\
\text { CACO3 } \\
(39086)\end{array}$ & $\begin{array}{c}\text { NITRO- } \\
\text { GEN } \\
\text { NITRATE } \\
\text { TOTAI } \\
\text { (MG/L } \\
\text { AS N) } \\
(00620)\end{array}$ & $\begin{array}{c}\text { NITRO- } \\
\text { GEN } \\
\text { NO2+NO3 } \\
\text { TOTAI } \\
\text { (MG/I } \\
\text { AS N) } \\
(00630)\end{array}$ & $\begin{array}{c}\text { NITRO- } \\
\text { GEN } \\
\text { NO2+NO3 } \\
\text { DIS- } \\
\text { SOIVED } \\
\text { (MG/ } \\
\text { AS N) } \\
(00631)\end{array}$ & $\begin{array}{c}\text { NITRO- } \\
\text { GEN } \\
\text { AMMONIA } \\
\text { DIS- } \\
\text { SOLVED } \\
\text { (MG/I } \\
\text { AS N) } \\
(00608)\end{array}$ \\
\hline \multirow{9}{*}{ 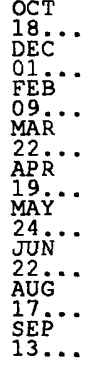 } & 8.0 & 92 & 15 & 0 & 12 & 0.060 & 0.060 & 0.060 & 0.020 \\
\hline & 10.0 & 90 & 8 & 0 & 6 & 0.060 & 0.060 & 0.060 & $<0.015$ \\
\hline & 11.4 & 97 & 9 & 0 & 7 & 0.100 & 0.100 & 0.100 & 0.020 \\
\hline & 8.8 & 101 & 9 & 0 & 7 & $-\infty$ & $-\cdots$ & $<0.050$ & $<0.015$ \\
\hline & 8.5 & 92 & 8 & 0 & 7 & -- & -- & $<0.050$ & 0.020 \\
\hline & B. 0 & 100 & 11 & 0 & 9 & --- & -- & $<0.050$ & 0.020 \\
\hline & 7.3 & 96 & 15 & 0 & 12 & -- & -- & $<0.050$ & 0.040 \\
\hline & 5.8 & 81 & 20 & 0 & 16 & --- & -- & $<0.050$ & 0.050 \\
\hline & 5.0 & 61 & 25 & 0 & 20 & 0.120 & 0.120 & 0.120 & 0.060 \\
\hline
\end{tabular}


POTEAO RTVER SUBBASIN

07247345 BLACK FORK AT HODGHN, OKLABOMA--CONTINUED WATER-QUALITY DATA, WATER YEAR OCTOBER 1994 TO SEPTEMBER 1995

\begin{tabular}{|c|c|c|c|c|c|c|c|c|c|}
\hline DATE & $\begin{array}{r}\text { NITRO- } \\
\text { GEN } \\
\text { AMMONIA } \\
\text { DIS- } \\
\text { SOLVED } \\
\text { (MG/L } \\
\text { AS NH4) } \\
(71846)\end{array}$ & $\begin{array}{r}\text { NITRO- } \\
\text { GEN } \\
\text { ORGANIC } \\
\text { TOTAL } \\
\text { (MG/ } \\
\text { AS N) } \\
(00605)\end{array}$ & $\begin{array}{c}\text { NITRO- } \\
\text { GEN AM- } \\
\text { MONAA + } \\
\text { ORGANIC } \\
\text { TOTAL } \\
\text { (MG/L } \\
\text { AS N) } \\
\{00625)\end{array}$ & $\begin{array}{c}\text { NITRO- } \\
\text { GEN } \\
\text { TOTAL } \\
\text { (MG/L } \\
\text { AS N) } \\
(00600)\end{array}$ & $\begin{array}{c}\text { PHOS- } \\
\text { PHORUS } \\
\text { TOTAL } \\
\text { (MG/L } \\
\text { AS P) } \\
(00665)\end{array}$ & $\begin{array}{c}\text { PHOS- } \\
\text { PHORUS } \\
\text { ORTHO, } \\
\text { DIS- } \\
\text { SOLVED } \\
\text { (MG/L } \\
\text { AS P) } \\
(00671)\end{array}$ & $\begin{array}{r}\text { PHOS- } \\
\text { PHATE, } \\
\text { ORTHO, } \\
\text { DIS } \\
\text { SOLVED } \\
\text { (MG/L } \\
\text { AS PO4) } \\
\text { (00660) }\end{array}$ & $\begin{array}{c}\text { SEDI- } \\
\text { MENT, } \\
\text { SUSS- } \\
\text { PENDED } \\
(\text { MG/L) } \\
(80154)\end{array}$ & $\begin{array}{r}\text { SEDI- } \\
\text { MENT, } \\
\text { DIS- } \\
\text { CHARGE, } \\
\text { SUS - } \\
\text { PENDED } \\
(\text { T/DAY } \\
(80155)\end{array}$ \\
\hline $\begin{array}{l}\text { OCT } \\
18 \\
\end{array}$ & 0.03 & 0.38 & 0.40 & 0.46 & 0.020 & $<0.010$ & -- & 4 & 0.12 \\
\hline $\begin{array}{l}\text { DEC } \\
01 . . .\end{array}$ & -- & 0.30 & 0.30 & 0.36 & 0.020 & $<0.010$ & --- & 6 & 3.6 \\
\hline $\begin{array}{l}\text { FEB } \\
09 . . .\end{array}$ & 0.03 & -- & $<0.20$ & -- & $<0.010$ & $<0.010$ & --- & 5 & 1.6 \\
\hline $\begin{array}{l}\text { MAR } \\
22 \ldots . .\end{array}$ & -- & 0.20 & 0.20 & 0.20 & 0.030 & $<0.010$ & -- & 4 & 1.1 \\
\hline $19 .$. & 0.03 & $-\infty$ & $<0.20$ & --- & 0.020 & $<0.010$ & --- & 6 & 5.0 \\
\hline $\begin{array}{l}\text { MAY } \\
24 . \ldots\end{array}$ & 0.03 & --- & $<0.20$ & -- & $<0.010$ & $<0.010$ & --- & 8 & 1.2 \\
\hline $22 \ldots$ & 0.05 & 0.36 & 0.40 & 0.40 & 0.030 & 0.020 & 0.06 & 7 & 0.36 \\
\hline $17 \ldots$ & 0.06 & 0.35 & 0.40 & 0.40 & 0.020 & $<0.010$ & $\cdots$ & 9 & 0.01 \\
\hline $13 \ldots$ & 0.08 & 0.44 & 0.50 & 0.62 & 0.050 & $<0.010$ & --- & 9 & 0.00 \\
\hline
\end{tabular}




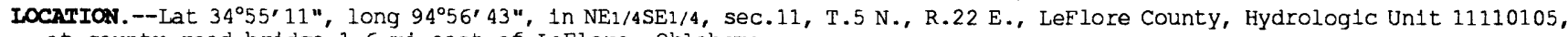
at county road bridge $1.6 \mathrm{mi}$ east of Leflore, Oklahoma.

DRATNAGE AREA. - $-270 \mathrm{mi}^{2}$.

PERIOD OF RECORD.--December 1991 to current year.

REMARKS.--Specific conductance, pH, water temperature, dissolved oxygen, and alkalinity were determined in the fleld. WATER-QUALITY DATA, WATER YEAR OCTOBER 1994 TO SEPTEMBER 1995

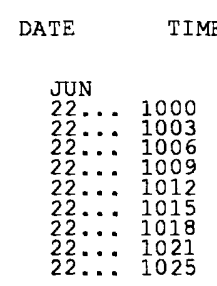

$\begin{array}{lccc}\text { SAMPLE } & & \text { BARO- } & \\ \text { IOC- } & & \text { METRIC } & \text { AGENCY } \\ \text { ATION, } & & \text { PRES- } & \text { COL- } \\ \text { CROSS } & \text { TEMPER- } & \text { SURE } & \text { LECTING } \\ \text { SECTION } & \text { ATTURE } & \text { (MM } & \text { SAMPLE } \\ \text { (FT FM } & \text { WATER } & \text { OF } & \text { (CODE } \\ \text { (FTANK) } & \text { (DEG C) } & \text { HG) } & \text { NUMBER) } \\ \text { (00009) } & (00010) & (00025) & (00027)\end{array}$
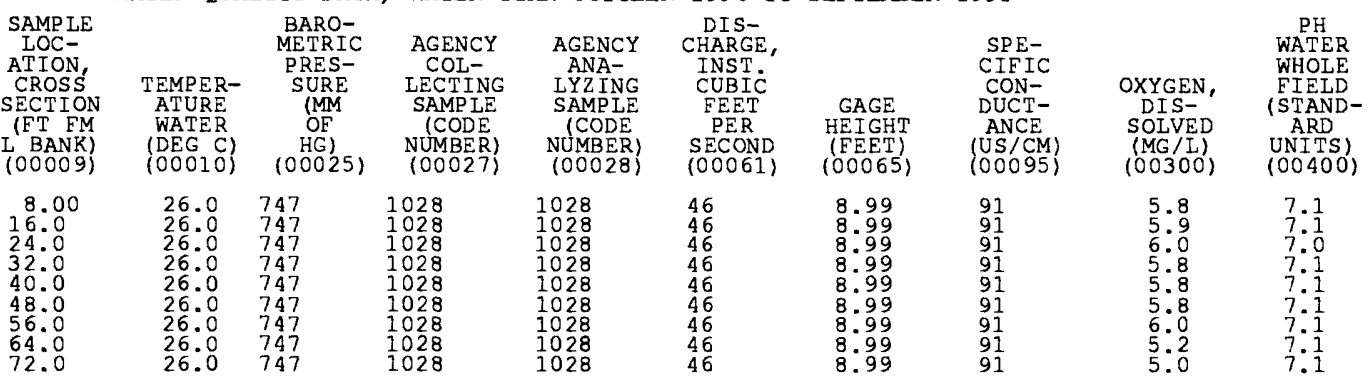

$\begin{array}{ll}8.99 & 91 \\ 8.99 & 91 \\ 8: 99 & 91 \\ 8: 99 & 91 \\ 8.99 & 91 \\ 8.99 & 91 \\ 8.99 & 91 \\ 8.99 & 91 \\ 8.99 & 91\end{array}$
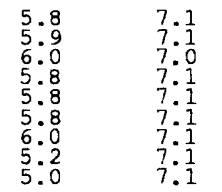

\begin{tabular}{|c|c|c|c|}
\hline DATE & TIME & $\begin{array}{c}\text { AGENCY } \\
\text { COL } \\
\text { LECTING } \\
\text { SAMPLE } \\
\text { (CODE } \\
\text { NUMBER } \\
\{00027\}\end{array}$ & $\begin{array}{c}\text { AGENCY } \\
\text { ANA } \\
\text { LYZING } \\
\text { SAMPLE } \\
\text { (CODE } \\
\text { NUMBER) } \\
(00028)\end{array}$ \\
\hline $\begin{array}{l}O C T \\
17 \ldots\end{array}$ & 1445 & 1028 & 80020 \\
\hline $29 \ldots$ & 1400 & 1028 & 80020 \\
\hline 09. & 1215 & 1028 & 80020 \\
\hline$\frac{1}{p} \cdots$ & 0940 & 1028 & 80020 \\
\hline $\begin{array}{l}1 \\
M A Y\end{array}$ & 0800 & 1028 & 80020 \\
\hline $25 \ldots$ & 0935 & 1028 & 80020 \\
\hline $2 \ldots$ & 0950 & 1028 & 80020 \\
\hline $17 \ldots$ & 0930 & 1028 & 80020 \\
\hline & 1430 & 1028 & 80020 \\
\hline
\end{tabular}

\begin{tabular}{|c|c|}
\hline $\begin{array}{c}\text { DIS- } \\
\text { CHARGE } \\
\text { INST. } \\
\text { CUBIC } \\
\text { FEET } \\
\text { PER } \\
\text { SECOND } \\
(00061)\end{array}$ & $\begin{array}{c}\text { SPE- } \\
\text { CIFIC } \\
\text { CON- } \\
\text { DUCT- } \\
\text { ANCE } \\
(\text { UN/CM) } \\
(00095)\end{array}$ \\
\hline 2.3 & 162 \\
\hline 170 & 89 \\
\hline 115 & 87 \\
\hline 72 & 95 \\
\hline 243 & 71 \\
\hline 51 & 93 \\
\hline 46 & 92 \\
\hline 0.85 & 147 \\
\hline 4.2 & 166 \\
\hline
\end{tabular}

P
WATE
WHOL
FIELD
(STAND
AR
UNTTS
(00400
7.3
7.3
$\star 7.1$
7.4
7.5
7.0
7.0
7.2
7.6
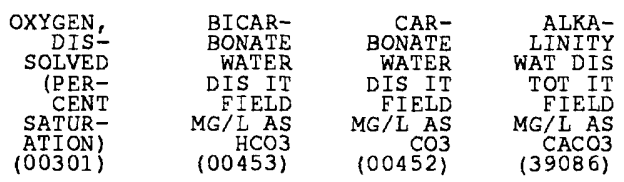

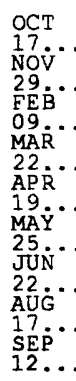

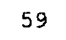

86

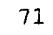

21

99

85

78

74

73

60

4.7

5.8

68

$100452\}$

(39086)

58
18
17
18
10
22
24
44
54

NITRO-
GEN,
AMMONIÁ

DATE SOLVED

AS (MG/

$\begin{array}{lc}\text { OCT } & (71846) \\ \text { IT } & 0.04\end{array}$

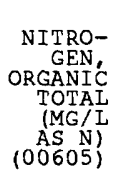

NITRO-

GEN AM

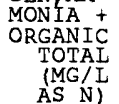

NITRO-
GEN,
TOTAL
(MG/L
AS N)

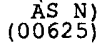

AS N )
$(00600)$

0.50

0.50

0.47

0.40

0.51

0.20

0.20

0.29

0.30

0.47

0.26

0.30

0.30

0.58

0.47

0.55

0.60

0.53

0.75

0.30

0.40

0.60

0.80

0.88

PHOS-
PHORUS
TOTAL
(MG /L
AS P)
$(00665)$
0.030
0.040
0.010
0.040
0.050
0.030
0.070
0.040
0.050

TEMPER-
ATURE
AIR
$(D E G)$ C
$(00020)$
23.0
12.0
12.0
24.5
8.5
19.5
28.0
28.5
23.0

TEMPER-
ATURE
WATER
(DEG C)
$(00010)$

BARO-
METRIC
PRES
PURE
SURM
(MF
OG)
$(00025)$
747
756
752
740
751
753
747
748
750

18.5

11.0

8.0

19.5

16.5

22.0

25.5

27.0

22.0
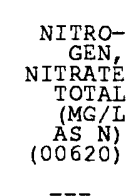

0.110

0.090

$--$

0.080

0.170

0.150

---

0.080

\section{NITRO-
GEN,
NO2+NO3
TOTAL
(MG $/$ L
AS N)
$(00630)$}

NITRONO2+NO3

SOLVED

(MG/I
AS N)

AS N)
$(00631)$

$<0.050$

0.110

0.090

$---$

0.080

0.170

0.150

$<0.050$

0.080

PHOS-
PHORUS

PHORUS

SOLVED

(MG/L

AS P
$(00671)$

0.010

0.020

0.010

-.-

0.080

PHOS-
PHATE,

PHATE,

DOIVED

AS (MG/L

AS PO4)
$(00660)$

0.03

0.06

0.03

0.010

0.03

0.030

0.09

0.020

0.06

$<0.010$

$<0.010$

$--$

$--$

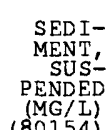

(MG/L)
$(80154)$

67

15

11

18

40

21

20

19

21

\section{NITRO- \\ GEN
AMMONIA
DIS-
SOLVED
(MG/L
AS N)}

AS N $)$
$(00608)$

0.030

$<0.015$

$<0.015$

0.030

0.040

0.040

0.070

0.050

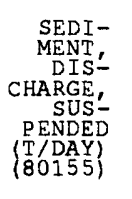

0.42

6.9

3.4

3.5

26

2.9

2.5

0.04

0.24 


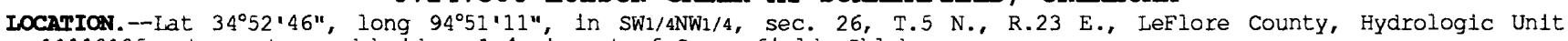
11110105 , at county road bridge, $1.4 \mathrm{mil}$ east of summerfleld, Oklahoma.

DRADIAGE AREA. $--71.6 \mathrm{mi}^{2}$.

PERIOD OF RBCORD.--December 1991 to current year.

REMARKS.--Specific conductance, pH, water temperature, dissolved oxygen, and alkalinity were determined in the fleld. WATER-QUALITY DATA, WATER YEAR OCTOBER 1994 TO SEPTEMBER 1995






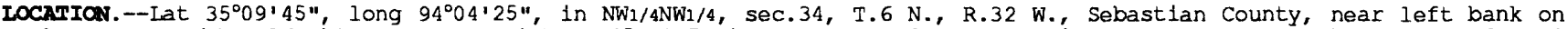
downstream side of bridge on State Highway $45,1.7 \mathrm{mt}$ south of Hackett, $2.0 \mathrm{mi}$ downstream from Elder Branch, $2.0 \mathrm{mi}$ upstream from small tributary, and $3.6 \mathrm{mi}$ upstream from Arkansas-oklahoma state 11ne.

DRATIAGE AREA.--147 $\mathrm{mi}^{2}$.

AVERACE DISCHAREE. -37 years, $144 \mathrm{ft}^{3} / \mathrm{s}$.

EXuREres.--April 1958 to current year: Maximum discharge 30,000 ft ${ }^{3} / \mathrm{s}$ May 14, 1968; no flow at times.

REMARKS.--Records good. Satellite telemeter at station.

Monthly and yearly discharge

\begin{tabular}{|c|c|c|c|c|c|}
\hline Month & $\begin{array}{l}\text { Tottal } \\
\left(\mathrm{ft}^{3} / \mathrm{s}\right)\end{array}$ & $\begin{array}{l}\text { Maximum } \\
\operatorname{dail}^{3} \mathrm{y} \\
(\mathrm{ft} / \mathrm{s})\end{array}$ & $\begin{array}{l}\text { Minimum } \\
\mathrm{daily}^{3} \mathrm{y} \\
(\mathrm{ft} / \mathrm{s})\end{array}$ & $\begin{array}{c}\text { Mean } \\
\left(\mathrm{ft}^{3} / \mathrm{s}\right)\end{array}$ & $\begin{array}{c}\text { Runoff } \\
\text { (acre-feet) }\end{array}$ \\
\hline October & 93.21 & 9.9 & .91 & 3.01 & 185 \\
\hline November & 18,533 & 4,590 & 13 & 618 & 36,760 \\
\hline December & 9,740 & 2,250 & 59 & 314 & 19,320 \\
\hline January & 16,828 & 4,680 & 66 & 543 & 33,380 \\
\hline February & 1,981 & 200 & 30 & 70.7 & 3,930 \\
\hline March & 3,882 & 1,080 & 27 & 125 & 7,700 \\
\hline April & 4,703 & 1,180 & 31 & 157 & 9,330 \\
\hline May & 11,271 & 4,730 & 23 & 364 & 22,360 \\
\hline June & 3,457 & 813 & 17 & 115 & 6,860 \\
\hline July & 727.5 & 243 & 6.0 & 23.5 & 1,440 \\
\hline August & 127.0 & 10 & 2.3 & 4.10 & 252 \\
\hline September & 121.4 & 8.9 & 1.9 & 4.05 & 241 \\
\hline Water year 1995 & $71,464.11$ & 4,730 & .91 & 196 & 141,700 \\
\hline
\end{tabular}


PERIOD OF RECORD.--October 1960 to September 1971, October 1975 to September 1978, October 1983 to current year.

REMRRS.--Specific conductance, pH, water temperature, dissolved oxygen, and alkalinity were determined in the field. WATER-QUALITY DATA, WATER YEAR OCTOBER 1994 TO SEPTEMBER 1995

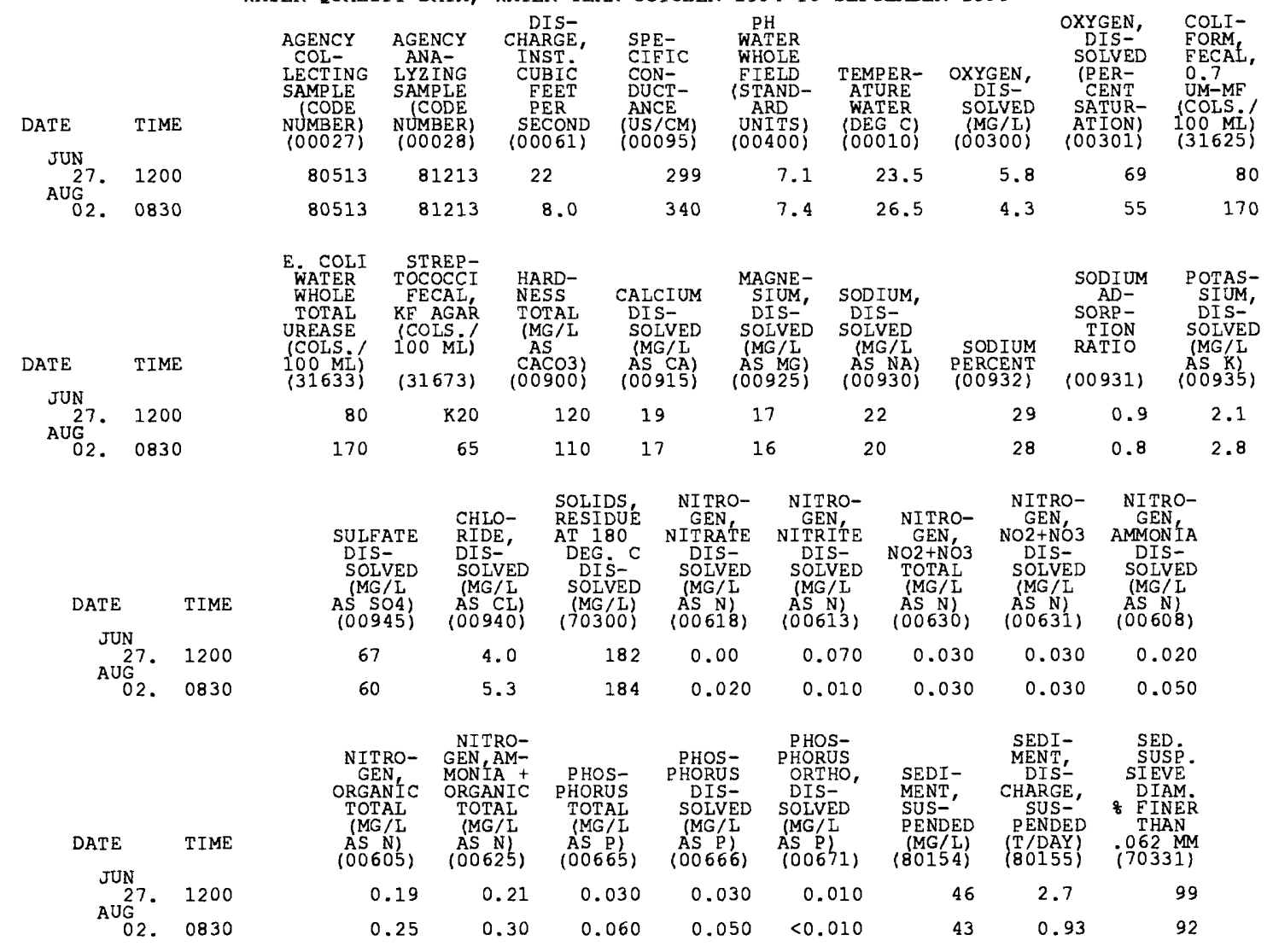




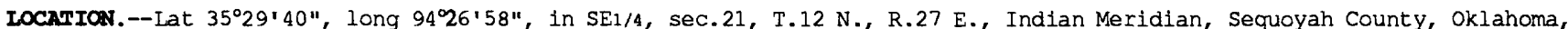
Hydrologic Un1t 11110104, on left bank $0.5 \mathrm{mi}$ west of Arkansas-Oklahoma State line, 500 ft downstream from Webbers Creek, $4.1 \mathrm{mi}$ south of Short, Oklahoma, $7.5 \mathrm{mi}$ southwest of Uniontown, Arkansas, and at mile 11.0 .

AVERAGE DISCEARGE.--51 years $(1930-36,1950-95), 532 \mathrm{ft}^{3} / \mathrm{s}$.

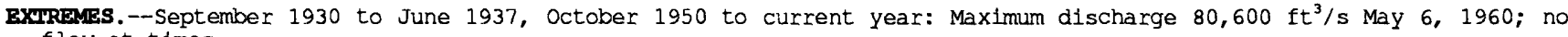
flow at times.

RBMRRS.--Records good. Prior to October 1992 published as 07250000 Lee Creek near Van Buren, Arkansas. Satellite telemeter at station.

Monthly and yearly discharge

\begin{tabular}{|c|c|c|c|c|c|}
\hline Month & $\begin{array}{l}\text { Total } \\
\left(\mathrm{ft}^{3} / \mathrm{s}\right)\end{array}$ & $\begin{array}{l}\text { Maximum } \\
\text { daily } \\
\left(\mathrm{ft}^{3} / \mathrm{s}\right)\end{array}$ & $\begin{array}{l}\text { Minimum } \\
\text { daily } \\
(\mathrm{ft} / \mathrm{s})\end{array}$ & $\begin{array}{c}\text { Mean } \\
\left(\mathrm{ft}^{3} / \mathrm{s}\right)\end{array}$ & $\begin{array}{c}\text { Runoff } \\
\text { (acre-feet) }\end{array}$ \\
\hline October & 784.7 & 40 & 2.5 & 25.3 & 1,560 \\
\hline November & 49,789 & 7,290 & 25 & 1,660 & 98,760 \\
\hline December & 23,898 & 4,910 & 213 & 771 & 47,400 \\
\hline January & 41,792 & 7,630 & 216 & 1,348 & 82,890 \\
\hline February & 10,362 & 782 & 217 & 370 & 20,550 \\
\hline March & 23,238 & 2,690 & 322 & 750 & 46,090 \\
\hline April & 34,439 & 6,630 & 219 & 1,148 & 68,310 \\
\hline May & 50,305 & 14,700 & 228 & 1,623 & 99,780 \\
\hline June & 22,801 & 4,410 & 144 & 760 & 45,230 \\
\hline July & 4,129 & 478 & 33 & 133 & 8,190 \\
\hline August & 537.9 & 70 & 1.2 & 17.4 & 1,070 \\
\hline September & 200.27 & 21 & .00 & 6.68 & 397 \\
\hline Water year 1995 & $262,275.87$ & 14,700 & 0.00 & 719 & 520,200 \\
\hline
\end{tabular}




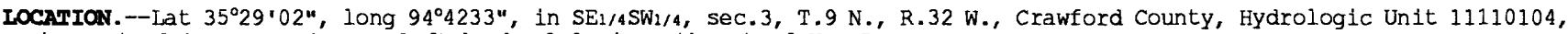
in control house at dam on left bank, $2.8 \mathrm{mi}$ northwest of van Buren.

DRATRAS AREA. $-432 \mathrm{mi}^{2}$.

PERIOD OF RECORD.--October 1992 to current year.

RERARRs.--Specif1c conductance, pH, water temperature, dissolved oxygen, and alkalinity were determined in the fleld. WATER-QUALITY DATA, WATER YEAR OCTOBER 1994 TO SEPTEMBER 1995

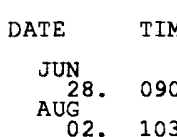

02. 1030

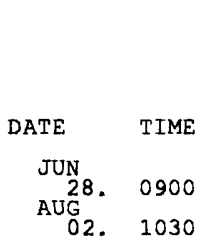

$$
\begin{array}{ll}
\text { DATE } & \text { TIME } \\
\text { JUN } & \\
28 . & 0900 \\
\text { AUG } & \\
02 . & 1030
\end{array}
$$
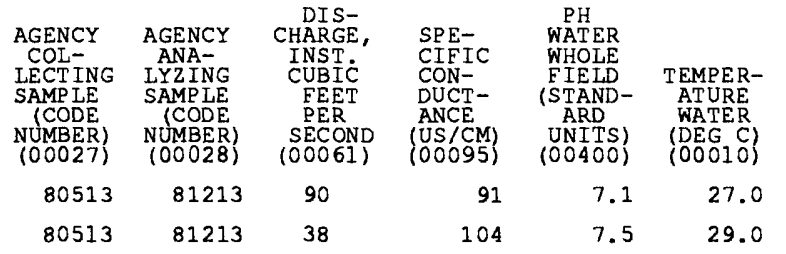

(DEG C)

$$
7.1
$$$$
7.5
$$

$$
27.0
$$$$
29.0
$$

\section{E. COLI STREP-}

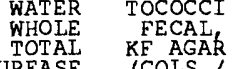

UREASE

(COLS:

(31633)

(COLS,'

$100 \mathrm{ML})$

(31673)
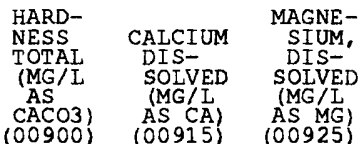

SODIUM,
DIS-
SOLVED
(MG/L
AS NA)
$(00930)$

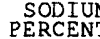

PERCENT

OXYGEN,
DIS-
SOLVED

$(\mathrm{MG} / \mathrm{L})$
$(00300)$

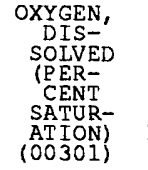

COLI-

FECA,

0.7

UM-MF,

(COLS.'

$100 \mathrm{ML})$
$(31625)$

K1 K9

$39 \quad 13$

1.5

1.8

5.8

2.5

7.7

5.6

99

K52

<1 KI

$40 \quad 13$
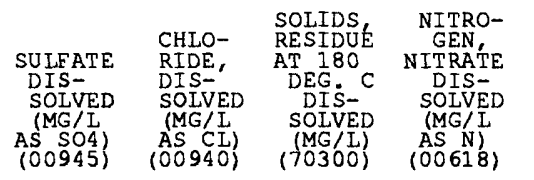

NITRO-
GEN,
NITRITE
DIS-
SOLVED
(MG/L
AS N)
(O0613)
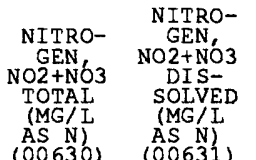

3.7

1.9

64

0.010

0.010

0.020

$<0.010$

$\sim$

0.020

$<0.020$

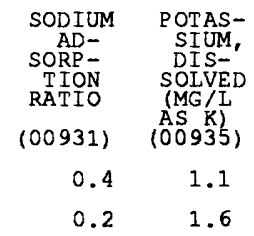

NITRO-
GEN, NITROAMMONIA GEN

DIS-

SOLVE

(MG/ I

AS N $)$ AS N)
$(00608) \quad(00605)$

\begin{tabular}{|c|c|c|c|}
\hline $\begin{array}{l}\text { NITRO- } \\
\text { GEN,AM- } \\
\text { MONIA + } \\
\text { ORGANIC } \\
\text { TOTAL } \\
(\text { MG/L } \\
\text { AS N) } \\
(00625)\end{array}$ & $\begin{array}{l}\text { PHOS- } \\
\text { PHORUS } \\
\text { TOTAI } \\
\text { (MG/I } \\
\text { AS P } \\
(00665)\end{array}$ & $\begin{array}{l}\text { PHOS- } \\
\text { PHORUS } \\
\text { DIS- } \\
\text { SOLVED } \\
\text { (MG/L } \\
\text { AS P) } \\
(00666)\end{array}$ & $\begin{array}{l}\text { PHOS- } \\
\text { PHORUS } \\
\text { ORTHO, } \\
\text { DIS- } \\
\text { SOLVED } \\
\text { (MG / } \\
\text { AS P) } \\
(00671)\end{array}$ \\
\hline 0.32 & 0.050 & $<0.020$ & 0.010 \\
\hline 0.42 & 0.020 & 0.020 & $<0.010$ \\
\hline
\end{tabular}

$0.020 \quad 0.30$

\begin{tabular}{|c|c|c|c|}
\hline $\begin{array}{l}\text { SEDI- } \\
\text { MENT, } \\
\text { SUS- } \\
\text { PENDED } \\
(\mathrm{MG} / \mathrm{L}) \\
(80154)\end{array}$ & $\begin{array}{c}\text { SEDI- } \\
\text { MENT, } \\
\text { DIS- } \\
\text { CHARGE, } \\
\text { SUS- } \\
\text { PENDED } \\
(\text { T } / D A Y) \\
(80155)\end{array}$ & $\begin{array}{c}\text { SED. } \\
\text { SUSP. } \\
\text { SIEVE } \\
\text { DIAM } \\
\text { FINER } \\
\text { THAN } \\
062 \mathrm{MM} \\
(70331)\end{array}$ & $\begin{array}{c}\text { CHLOR-A } \\
\text { PHYTO- } \\
\text { PLANK- } \\
\text { TON } \\
\text { CHROMO } \\
\text { FLUOROM } \\
\text { (UG/L) } \\
(70953)\end{array}$ \\
\hline 23 & 5.6 & 88 & 1.00 \\
\hline 19 & 1.9 & 83 & $\sim-$ \\
\hline
\end{tabular}

0.040

0.38 $\begin{array}{cc} & \\ \text { DATE } & \text { TIME } \\ \text { JUN } & \\ \text { 28. } & 0900 \\ \text { AUG } & \\ \text { 02. } & 1030\end{array}$ 
07250550 ARKANSAS RIVER AT JAMES W. TRTMBLE LOCK AND DAM NEAR VAN BUREN, ARKANSAS

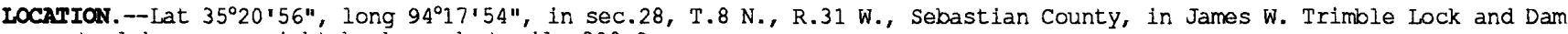
control house on right bank, and at mile 308.9 .

DRAnNace AREA.--150,547 $\mathrm{mi}^{2}$, of which $22,241 \mathrm{mi}^{2}$ is probably noncontributing.

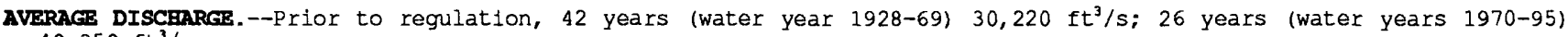
$40,350 \mathrm{ft}^{3} / \mathrm{s}$.

EXcremes. --October 1927 to current year: Maximum discharge 850,000 ft $3 / 5$ May 12, 1943; no flow Nov. 2, 1975, Feb. 1, 1981, Oct. 17, 1987, Dec. 9, 1989, Nov. 11-12, 1993, Jan. 9, 13, 1994.

REMarks.--Records good except for discharges below $10,000 \mathrm{ft}^{3} / \mathrm{s}$, which are fair. Prior to October 1969 , published as 07250500 Arkansas River at Van Buren. Beginning Apr. 26, 1970, daily discharge computed from relation between discharge, head, and gate openings. Flow regulated by many locks, dams, and reservoirs upstream. Satelilte telemeter at station.

Monthly and yearly discharge

\begin{tabular}{|c|c|c|c|c|c|}
\hline Month & $\begin{array}{c}\text { Total } \\
\left(\mathrm{ft}^{3} / \mathrm{s}\right)\end{array}$ & $\begin{array}{l}\text { Maximum } \\
\operatorname{dail} \mathrm{yy} \\
\left(\mathrm{ft}^{3} / \mathrm{s}\right)\end{array}$ & $\begin{array}{l}\text { Minimum } \\
\text { daily } \\
\left(\mathrm{ft}^{3} / \mathrm{s}\right)\end{array}$ & $\underset{\left(\mathrm{ft}^{3} / \mathrm{s}\right)}{\operatorname{Mean}}$ & $\begin{array}{c}\text { Runoff } \\
(\mathrm{acre}-\mathrm{feet})\end{array}$ \\
\hline October & 165,539 & 20,300 & 0.00 & 5,340 & 328,300 \\
\hline November & $2,154,471$ & 105,000 & 951 & 71,820 & $4,273,000$ \\
\hline December & $1,429,560$ & 86,300 & 7,660 & 46,110 & $2,836,000$ \\
\hline January & $1,140,540$ & 99,500 & 7,350 & 36,790 & $2,262,000$ \\
\hline February & 603,630 & 44,700 & 3,080 & 21560 & $1,197,000$ \\
\hline March & $1,514,600$ & 75,000 & 17,000 & 48,860 & $3,004,000$ \\
\hline April & $1,594,580$ & 112,000 & 8,080 & 53,150 & $3,163,000$ \\
\hline May & $4,545,000$ & 186,000 & 109,000 & 146,600 & $9,015,000$ \\
\hline June & $5,746,000$ & 290,000 & 147,000 & 191,500 & $11,400,000$ \\
\hline July & $3,240,100$ & 157,000 & 39,100 & 104,500 & $6,427,000$ \\
\hline August & $1,587,600$ & 69,200 & 35,400 & 51,210 & $3,149,000$ \\
\hline September & 324,222 & 30,500 & 92 & 10,810 & 643,100 \\
\hline Water year 1995 & $24,045,842$ & 290,000 & 0.00 & 65,880 & $47,690,000$ \\
\hline
\end{tabular}


07250550 ARKANSAS RIVER AT JAMES $W$. TRIMBLE LOCK AND DAM MEAR VAN BUREN--CONTINOED

PERIOD OF RECORD.--October 1969 to September 1992, June to September 1995. Prior to October 1969, publ1shed as 07250500 Arkansas River at Van Buren.

REMARKS.--Speciflc conductance, $\mathrm{pH}$, water temperature, dissolved oxygen, and alkalinity were determined in the fleld. WATER-QUALITY DATA, WATER YEAR OCTOBER 1994 TO SEPTEMBER 1995

\begin{tabular}{|c|c|c|c|c|c|c|c|c|c|c|}
\hline DATE & TIME & $\begin{array}{l}\text { AGENCY } \\
\text { COL } \\
\text { IECTING } \\
\text { SAMP IEE } \\
\text { (CODE } \\
\text { NUMBER } \\
\{00027)\end{array}$ & $\begin{array}{l}\text { AGENCY } \\
\text { ANA- } \\
\text { LYZING } \\
\text { SAMP IE } \\
\text { (CODE } \\
\text { NUMBER) } \\
(00028)\end{array}$ & $\begin{array}{c}\text { DIS- } \\
\text { CHARGE, } \\
\text { INST. } \\
\text { CUBIC } \\
\text { FEET } \\
\text { PER } \\
\text { SECOND } \\
(00061)\end{array}$ & $\begin{array}{l}\text { SPE- } \\
\text { CIFIC } \\
\text { CON- } \\
\text { DUCT- } \\
\text { ANCE } \\
\text { (US/CM) } \\
(00095)\end{array}$ & $\begin{array}{c}\text { PH } \\
\text { WATER } \\
\text { WHOLE } \\
\text { EIELD } \\
\text { (STAND- } \\
\text { ARD } \\
\text { UNITS) } \\
(00400)\end{array}$ & $\begin{array}{l}\text { TEMPER- } \\
\text { ATURE } \\
\text { WATER } \\
(\text { DEG C }) \\
(00010)\end{array}$ & $\begin{array}{c}\text { OXYGEN, } \\
\text { DIS- } \\
\text { SOLVED } \\
(\text { MG /L) } \\
(00300)\end{array}$ & $\begin{array}{c}\text { OXYGEN, } \\
\text { DIS- } \\
\text { SOLVED } \\
\text { (PER- } \\
\text { CENT } \\
\text { SATUR- } \\
\text { ATION) } \\
(00301)\end{array}$ & $\begin{array}{l}\text { COLI- } \\
\text { FORM, } \\
\text { FECAL, } \\
0.7 \\
\text { UM-MF } \\
(\mathrm{COLS}, \\
100 \mathrm{ML}) \\
(31625)\end{array}$ \\
\hline 26 . & 1600 & 80513 & 81213 & 162000 & 355 & 7.6 & 24.5 & 9.8 & 119 & 69 \\
\hline 01. & 0915 & 80513 & 81213 & 44400 & 510 & 8. 6 & 29.0 & 7.4 & 98 & -- \\
\hline DATE & TIME & $\begin{array}{c}\text { E. COLI } \\
\text { WATER } \\
\text { WHOLE } \\
\text { TOTAL } \\
\text { UREASE } \\
\text { (COLS.' } \\
\text { 100 ML) } \\
\text { (31633) }\end{array}$ & $\begin{array}{c}\text { STREP- } \\
\text { TOCOCCI } \\
\text { FECAL } \\
\text { KF AGA } \\
\text { (COLS.' } \\
100 \mathrm{ML}) \\
(31673)\end{array}$ & $\begin{array}{c}\text { HARD- } \\
\text { NESS } \\
\text { TOTAL } \\
\text { (MG/L } \\
\text { AS } \\
\text { CACO3) } \\
(00900)\end{array}$ & $\begin{array}{l}\text { CALCIUM } \\
\text { DIS- } \\
\text { SOLVED } \\
\text { (MG/L } \\
\text { AS CA }) \\
(00915)\end{array}$ & $\begin{array}{c}\text { MAGNE- } \\
\text { SIUM, } \\
\text { DIS- } \\
\text { SOLVED } \\
\text { (MG/L } \\
\text { AS MG) } \\
(00925)\end{array}$ & $\begin{array}{l}\text { SODIUM, } \\
\text { DIS- } \\
\text { SOLVED } \\
(\mathrm{MG} / \mathrm{L} \\
\text { AS NA) } \\
(00930)\end{array}$ & $\begin{array}{l}\text { SODIUM } \\
\text { PERCENT } \\
(00932)\end{array}$ & $\begin{array}{l}\text { SODIUM } \\
\text { AD- } \\
\text { SORP- } \\
\text { TION } \\
\text { RATIO } \\
(00931)\end{array}$ & $\begin{array}{l}\text { POTAS- } \\
\text { SIUM, } \\
\text { DIS- } \\
\text { SOLVED } \\
\text { (MG/L } \\
\text { AS K) } \\
(00935)\end{array}$ \\
\hline 26. & 1600 & K41 & 96 & 120 & 35 & 7.4 & 32 & 36 & 1 & 4.2 \\
\hline 01. & 0915 & - & K6 & 120 & 37 & 7.5 & 50 & 46 & 2 & 4.0 \\
\hline
\end{tabular}

$$
\begin{array}{cc}
\text { DATE } & \text { TIME } \\
\text { JUN } & \\
\text { AUG } & 1600
\end{array}
$$$$
01.0915
$$

DATE TIME

$\begin{array}{cc}\text { JUN } & \\ 26 . & 1600 \\ \text { AUG } & 0915 \\ 01 . & 0915\end{array}$
K6
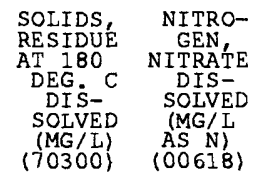

222

290

0.120

76

NITRO-

GEN'

DIS-

SOLVED

AS N)
$(00613)$

0.010

0.010

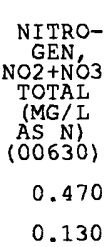

NITRO- NITROGEN, GEN, NO2+ $\mathrm{NO}^{2}$ AMMONIA SOIVED SOLVED (MG/L (MG/L AS N) ASN)
$(00631) \quad(00608)$

$\begin{array}{lll}30 & 42 & 222 \\ 37 & 76 & 290\end{array}$

0.130

0.470

0.030

0.130

0.030
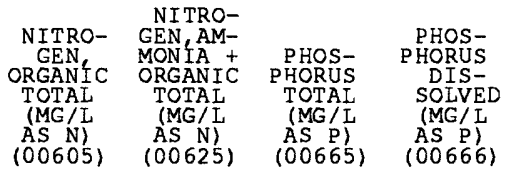

PHOS-
PHORUS

ORTHO,

DOIS-

(MG/I

AS P)
$(00671)$

AS P)
$(00665)$

0.60

0.63

0.160

0.070

0.070

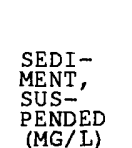

SEDI-

SEDI- SED.

DIS'- SIEVE

HARGE,

SUS-

T/DAY

(B0154) (B0155) (70331)
0.49
0.52
0.130

0.040

0.040

14764300

$53 \quad 6350$
4.0 